
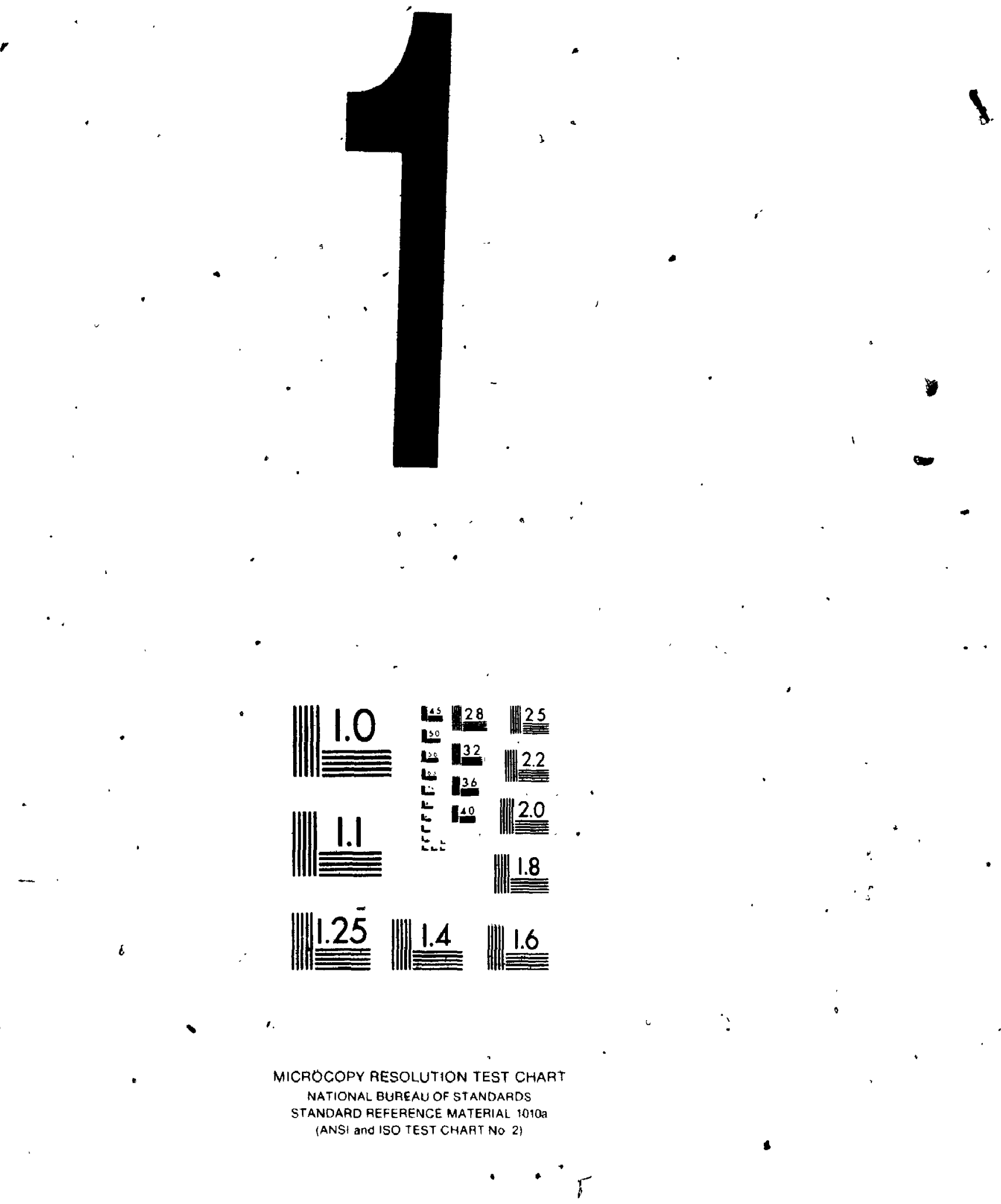


\section{National Library - Bibliothèque nationale \\ of Canada \\ du Canada}

Canadian Theses Service

Services des theses canadiennes

\section{CANADIAN THESES}

Ottawa, Canada

K1A ON4
4

\section{THĖSES CANADIENNES}

\section{NOTICE}

The quality of this microfiche is heavily dependent upon the quality of the original thesis submitted for microfilming. Every effort has been made to ensure the highest quality of reproduction possible.

If pages are missing, contact the university which granted the degree:

Some pages may have indistinct print especially if the original pages were typed with a poor typewriter ribbon or if the university sent us an inferior photocopy.

Previously copyrighted materials (journal articles, published tests, etc.) are not filmed.

Reproduction in full or in part of this film is governed by the Canadian Copyright Act, R.S.C. 1970, g C+30.

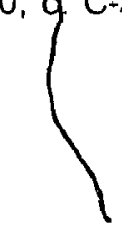

La qualité de cette microfiche dépend grandement de la qualité de la these soumise au microfilmage Nous avons tout falt pour assurer une qualité supérieure de reproduction

S'il manque des pages, veuillez communiquer avec l'université qui a conféré le grade.

La quaftlé d'impression de certaines pages peut laisser à désirer. surtout si les pages originales ont éte dactylographiees à l'aide d'uñ ruban usé ou sı l'unıversité nous a fait parvenır une photocopie de qualite inférieure

Les documents qui font déjà l'ọbjet d'un droll d'auteur (articles de revue, examens publiés, etc.) ne sont pas microfilmés.

La reproduction, même partielle, de ce microfilm esı soumise a la Loi canadienne sur le droit d'auteur, SRC 1970, e C-30

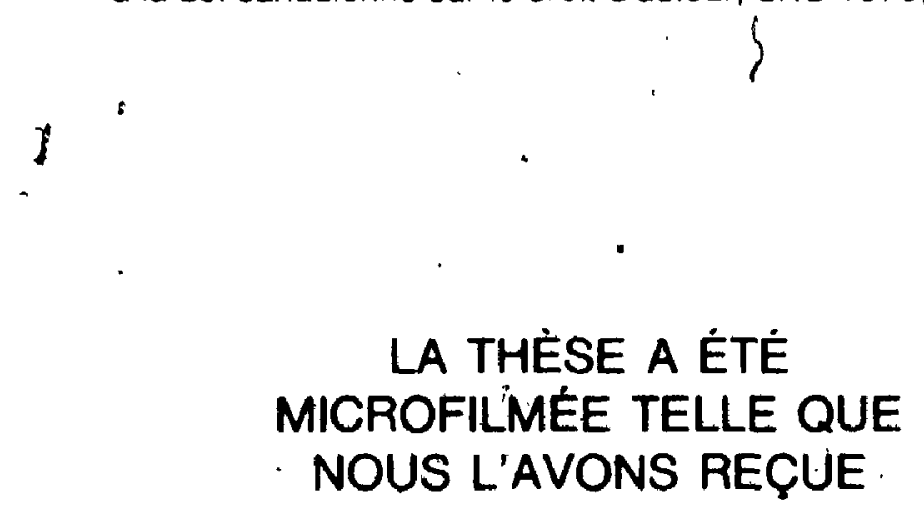

THIS DISSERTATTION

HAS BEEN MICROFILMED EXACTLY AS RECEIVED

\section{LA THÉSE A ÉTÉ MICROFILMEE TELLE QUE NOUS L'AVONS REÇUE}




\title{
Measurements of Mean Velocities : and Reynolds Stresses in a Circulation Controlled Flow
}

\author{
Jean F. Agiomavritis \\ BASc.(University of Ottawa) \\ h \\ A Thesis submitted to the Faculty of Graduate \\ Studies and Research in pdrtial fulfulment \\ of the requirements for the degree \\ (t) \\ of Master of Engineering
}

DEPARTMENT OF MECHANICAL AND AERONAUTICAL ENGINEERING

CARLETON UNIVERSITY

OTTAWA, ONTARIO

- CANADA

(C)

December 1985 


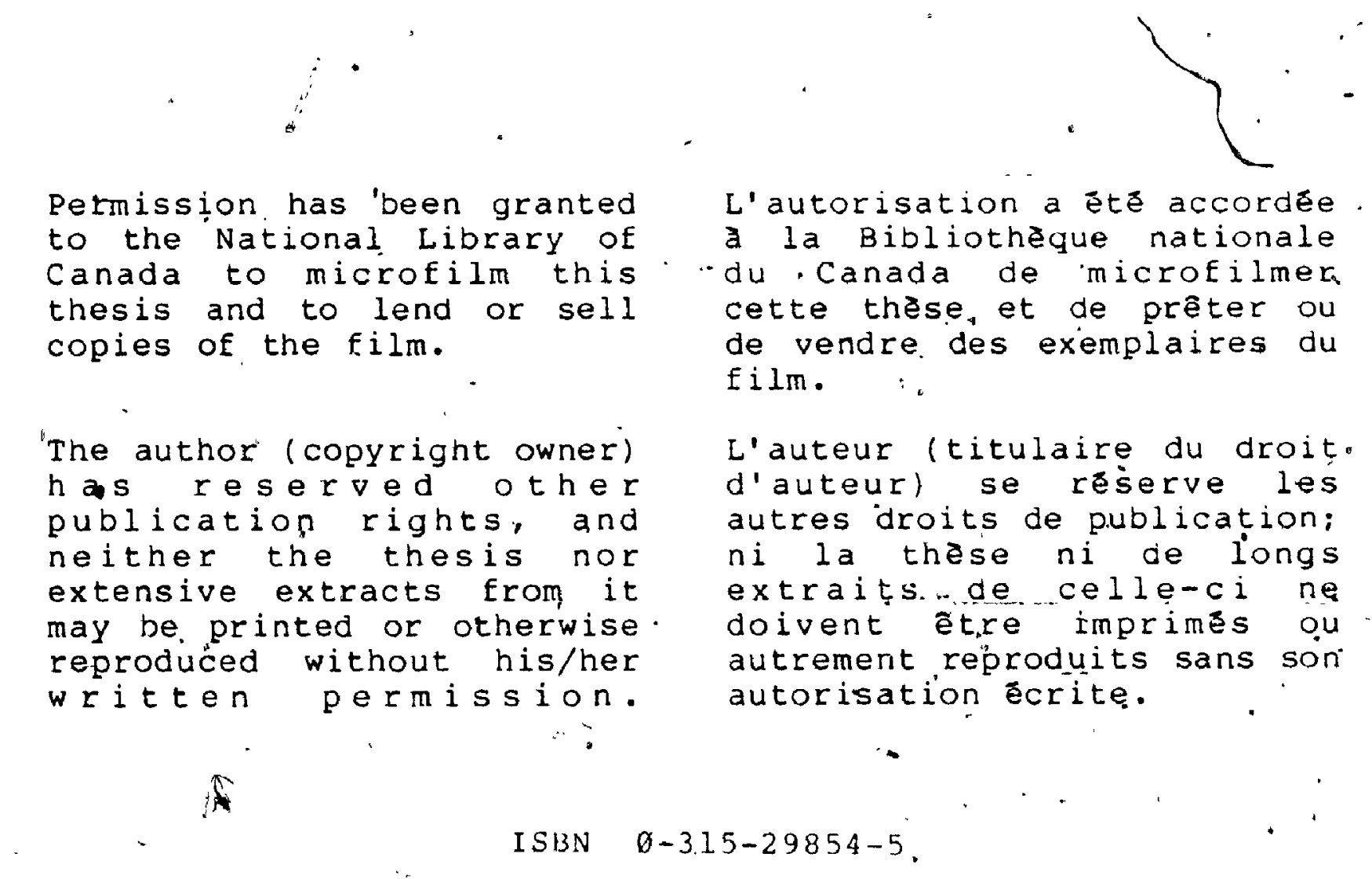




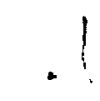

xvi :

The undersigned recommend to the Faculty of Graduate Studies and Research acceptance of the thesis;

\section{Measurements of Mean Velocitics and Reynolds Stre`sses}

in a Circulai $n$ Controlled Flow

submitted by Jean Agiomavritis, in partial fullfilment of the requirements for the degree of Master of Engineering.
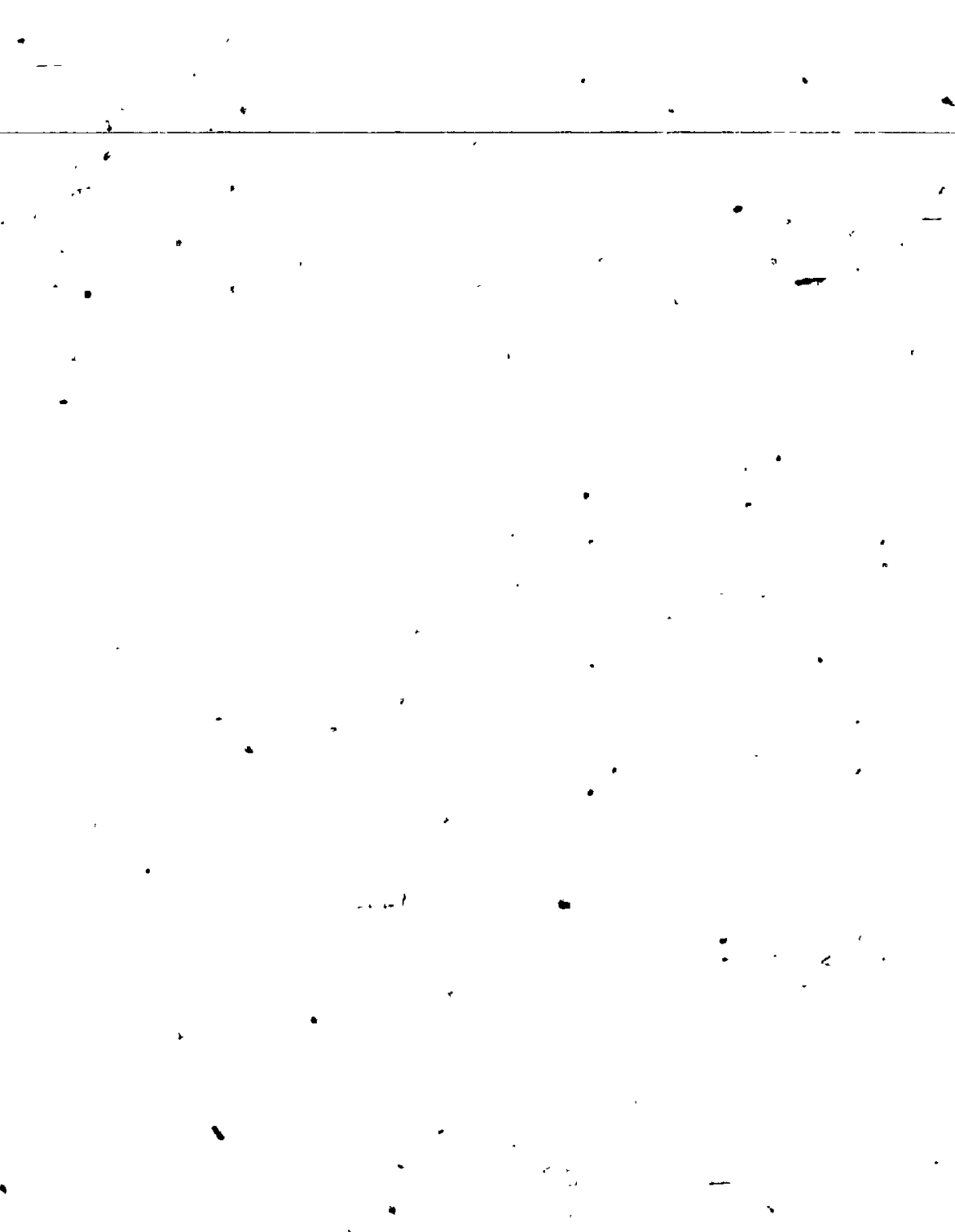


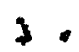 \\ Abstract}

This thesis is concerned with measurements of mean flow and turbulence parameters In the hear flow downstream of a slot, from which air is injected tangentially to the surfar. to provide boundary layer control.

- A hot-wire sigtral analysis has been derived and tested experimentally on a plane wall jet apparatus to determine its applicability and limitations. Experimental results for meat: velocity and Reynolds stresses obtained from the plane wall-jet apparatus agree with thw we of other investigators. Modifications to an existing Boundary LayerControl apparatus hu... been made and necessary software for a Data Acquisition System has been developed in

- collect data and control the experiments.

The two-dimensional characteristics of the flow in the modified Boundary Layer Control apparatus have been improfved using boundary layer suction and the quality of the flow was checked by static pressure measuręments, spanwise mean velocity hot-wire.measurements and by two flow visualization schemes. Mean velocity and Reynolds stress hot-wire data have been obtained for three different cylinder momentum coefficients, with the plane wall momentum coefficient and the tunnel free stream velocity held constant. All data have been analysed using a simplified version of the hot-wire signal 'analysis.

The measurements have extended the data available for flowfields similar to that observed on the rounded trailing edge of airfoils which utilize tangential blowing for boundary layer and circulation control. 


\section{Acknowledgements '}

I would like to extend my deepest and most sincere appreciation to my thesis supervisor, Professor R.J. Kind, for his continued interest, encouragernent and guidance provided during this research. In addition, I would like to thank the chairman, Dr. H.I.H Saravanamuttoo and the Department of Mechanical and Aeronautical Engineering,. Carleton Uniwersity, for their generous financial support which helped me in carrying out this research.

A special thanks is given to Mr. T. Goodwin and the staff of the Mechanical Engineering Technical Centre for their assistance in building the apparatus.

Special thanks, also to my colleague, Sean A. Bittle for his constant support provided throughout my studies at Carleton, and to Dr: F.M. Yowakim for making available his hot-wire signal analysis.

I would also like to thank my friend Anastasia Avramides for her continurous support and Harvey Smith for his patience with the word processing of this thesis. 


\section{Table of Contents}

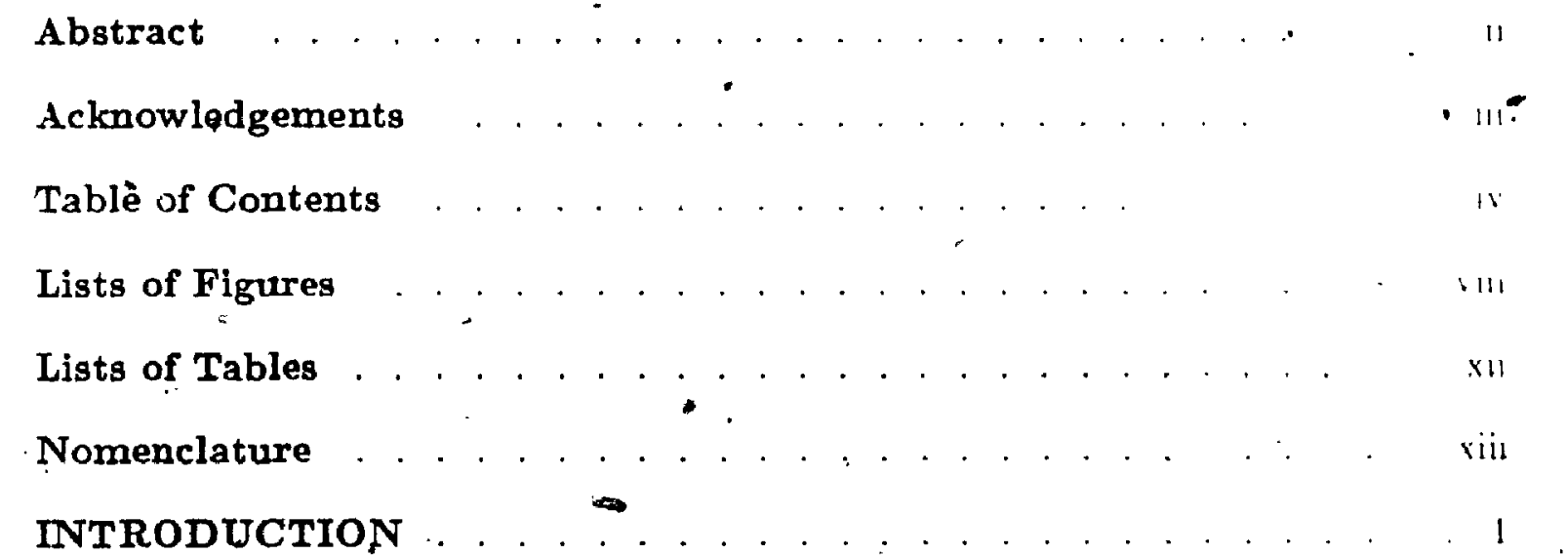

1.1 Backgrouind . . . . . . . . . . . . . . . . . . . . . . . . . . . 1

1.2 Nature of Flow .. . . . . . . . . . . . . . . . . . . . . . . . . . 2

1.3 Previous résearch

1.4 Purpose of the Present Study . . . . . . . . . . . . . . . . . . . . . . . . 4

2 DESCRIPTION OF APPARATUS . . . . . . . . . . . . . . . . . . 5

2.1 Wall-Jet Apparatus .. . . . . . . . . . . . . . . . . . . . . . . . 5

- 2.2 Boundary Layer Control Apparatus . . . . . . . . . . . . . . . . . 6

-2.2.1 Wind Tunnel . $\dot{-}^{\circ}$. . . . . . . . . . . . . . . . . . . . 6

2.2.2 Test Section : . . . . . . . . . . . . . . . . . . . f f

2.2.3 Plane Wall and Cylinder Wall Jets . . . . . . . . . . . . . . . 6

2.2.4 Porous Endwalls . . . . . . . . . . . . . . . . . . . . . . . . i

2.2 .5 Forebody . . . . . . . . . . . . . . . . . . . . . . . . . . 7

2.2.6 Traversing Gear . . . . . . , . . . . . . . . . . . . . . . . ×

2.2.7 Orifice-Plate Flowmeters . . . . . . . . . . . . . . . : . . . . . . . .

2.2.8 Manometers and Pressure Transducer . . . . . . . . . . . . . !

2.2 .9 Blowers . . . . . . . . . . . . . . . . . . . . . . . 10

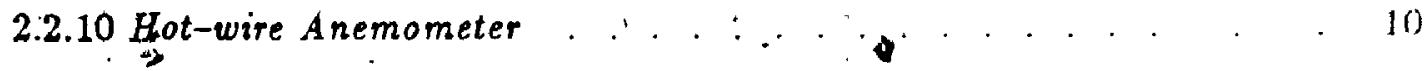

2.2.1 Calibration Apporatus . . . . . . . . . . . . . . . . . . . . 11

2.2.12 Automatic Data Acquisition and Cgntrol System (ADACS)' 
3 EXPERIMENTAL PROCEDURES

3.1 Experimentation on the Plane Wall-Jet Apparatus . . . . . . . . . . 13,

3.1.1 Familiarization with Hot-Wire Measuring techniques . . . . . . 13

/3.1.2 Development and Testing of ADACS Computer Programs . . . . 14

3.1.3 Hot-Wire Calibration and Linearization Methods . . . . . . . . 15

3.1.4 Determination of the Sensitivity Coefficients . . . . . . . . . . 17

3.2 Experimentation on the B.L.C. Apparatus . . . . . . . . . . . . . . . 18

3.2.1 Mass Flow Measurements . . . . . . . . . . . . . . . . . . . . . . 18

3.2.2 End-Wall Boundary Layer Control . . . . . . . . . . . . . . 20

3.2.3 Flow Quality Checks .. . . . . . . . . . . . . . . . . . . . 21

- 3.2.4 Repeatability and Operating Conditions . . . . . . . . . . . . 23

4 HOT-WIRE-SIGNAL ANALYSIS

4.1 Introduction . . . . . . . . . . . . . . . . . ... . . . . . . . 8

4.2 Derivation of Hot-Wire Equations for 3-D Flows _. . . . . . . . . . . . . 28

4.2.1 Determination of Mean and Fluctuating Quantities at Different $(\psi, \alpha)$. . . . . . . . . . . . . . . . . . . . . 32

4.3 Evaluation of the Hot-Wire Signal Analysis . . . . . . . . . . . . . . 39

4.4 Error Analysis . . . . . . . . . . . . . . . . . . . . . . . . . 42

- 4.4.1 Effects of " $v$ " on Reynolds Stress Calculations. . . . . . . . . . . . 42

4.4.2 Effects of Probe Spatial Resolution . . . . . . . . . . . . . . 43

4.4.3 Effects of Signal Sampling Errors . . . . . . . . . . . . . . . 43

5 RESULTS AND DISCUSSION . .. . .. . . . . . . . . . . . . . . . 44

5.1 Results from the Plane Still Air Wall-Jet Apparatus . . . . . . . . . . 4

5.2 Results from B.L.C. Apparatus .. . . . . . . . . . . . . . . . . . 46

$\therefore \quad 5.2 .1$ Flow Quality . . . . . . . . . . . . . . . . . . . . . . . 46

5.2.2 Mean Velocity and Reynolds Stress Measurements . . . . . . . 48

5.3 Discussions :. . . . . . . . . . . . . . . . . . . . . . . . . . 50

5.3.1 Reynolds Normal Stresses . . . . . . . . . . . . . . . . . . 50

5.3.2 Reynolds Shear Stresses . . . . . . . . .. . . . . . . . . . . 52

5.4 Conclusions ' . . . . . . . . . . . . . . . . . . . . . . . . . 54

5.5 Recommendations for Future Work . . . . . . . . . . . . . . . . . . 55 $\because ;$ 


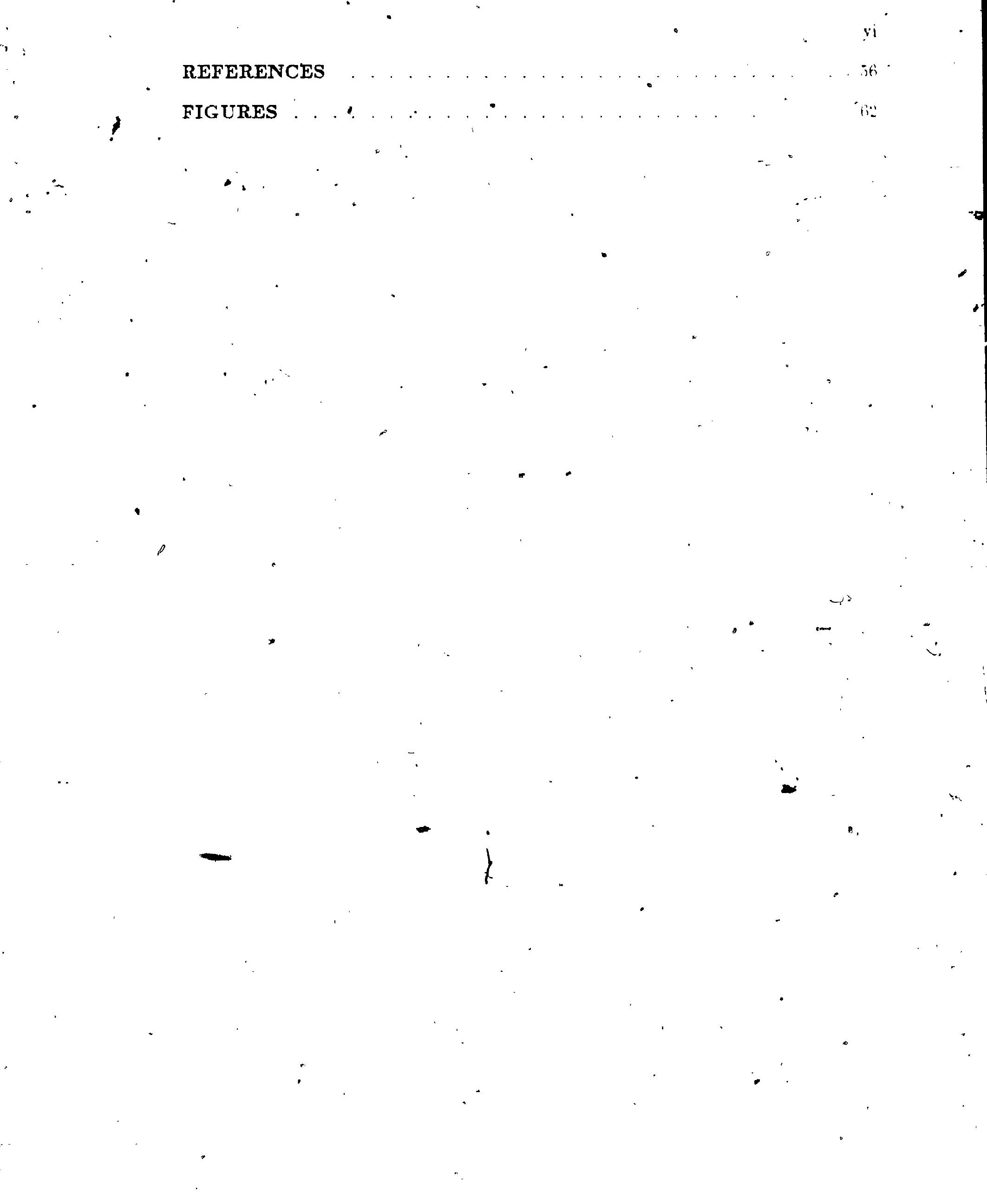




\section{APPENDIGES}

Appendi: - A Traversing Gear Design.

ippendix - B Computer Programs for Calibration of Hot-Wire Probes.

Appendix - C Computer Program, MFLOW

Appendix - D ADACS Computer Programs M\&RMS and U\&RMS

Appendix - E Hot-Wire Signal Analysis Programs.

Appendix - F Detailed Derivation of Hot-wire Signal Analysis.

Appendix - G Tabulated Results (B.L.C. Apparatus). 


\section{List of Figures}

Figure

1. Flow around a Circulation-Controlled Airfoil

2. Tangential Blowing over a Rounded Trailing Edge Airfoil

3. Flow near an Injection Slot

4. Possible Types of Flow Development Downstream of

an Injection Slot

5. Nomenclature for Cylinder Wall-Jet Velocity Profile

6. Photograph of the Plane Wall-Jet Apparatus

7. Photograph of the Calibration Apparatus

8. Boundary Layer Control Apparatus (B.L.C.), with Forebody

in place

9. Schematic Diagram of Blowing and Suction System

10. Traverse Gear Schematic Diagram (B.L.C. Apparatus)

11. Photograph of the Traversing Gear in place

12. Photograph of the Rear-Side of the B.L.C. Apparatus

13. Schematic Diagram of the Instrumentation

.14. Nomenclature for the Plane Still-Air Wall-Jet Velocity

Profle and'Probe Oriéntation during Measurements

15. Schematic Diagram of the Calibration Apparatus

16. Probe Orientation for. $k, h$ Measurements

17. Cylinder and Plane Wall-Jet Slot Calibration Cuive (B.L.C. Apparatus)

- 18. Probe Orientation for Measurements in the B.L.C.

Apparatus ,

19. Hot-Wire Probe Coordinate System and Nomenclature

20. Wall-Jet Mean Velocity Profiles, Plane Still-Air Wall-Jet

21. Wall-Jet Mean Velocity Profiles, Plane Still-Air Wah-Jet

22. Wall-Jet Longitudinàl Turbulence Profilè, Outer Layer ? Plane Still-Air Wall-Jet •

23. Normal Stress Profiles, Plane Still-Air Wall-Jet * $x / t=93.23$

24. Wall-Jet Shear Stress Profiles, Plane Still-Air Wall-Jet 
25. Shear Stress Profiles

26. Wall-Jet Growth,Plane Still-Air Wall-Jet

27. Spanwise Distribution of Maximum Total Pressürè in Wall-Jet on Cylinder in Still Air

28. Surface Static Pressure Distribution on the Cylinder in Still Surroundings

23. Forebody and Cylinder Static Pressure Distribution

30. Spanwise Static Pressure Distribution Downstream of the Plane Wall-Jet (B.L.C. Apparatus)

31. Flow Visualization, Effect of Boundary Layer Suction

$C_{\mu c}=0.1 ; C_{\mu w}=0.11 ; U_{\infty}=21 \mathrm{~ms}^{-1}$

32. Flow Visualization, Effect of Boundary Layer Suction

$C_{\mu c}=0.2 ; C_{\mu w}=0.11 ; U_{\infty}=21 \mathrm{~ms}^{-1}$

33. Flow Visualization, Effect of Boundary Layer Suction

$C_{\mu e}=0.22 ; C_{\mu w}=0.11 ; U_{\infty} \doteq 21 \mathrm{~ms}^{-1}$

34. Surface Flow Visualization

$C_{\mu c}=0.1 ; C_{\mu w}=0.11 ; U_{\infty}=21 \mathrm{~ms}^{-1}$

35. Surface Flow Visualization -.

$C_{\mu c}=0.2 ; C_{\mu w}=0.11 ; U_{\infty}=21 \mathrm{~ms}^{-1}$

36. Surface Flow Visualization

i $C_{\mu c}=0.22 ; C_{\mu w}=0.11 ; U_{\infty}=21 \mathrm{~ms}^{-1}$

37. Surface Flow Visualization

/ $\quad C_{\mu c}=0.22 ; C_{\mu w}=0.0 ; U_{\infty}=-0 \mathrm{~ms}^{-i}$

38. Spanwise Mean Velocity Profiles, Downstrean of the Cylinder

Slot, B.L.C. Apparatus (Normal Probe)

$C_{\mu c}=0.1 ; C_{\mu w}=\dot{0} .11 ; U_{\infty}=21 \mathrm{~ms}^{-1}$.

39. Spanwise Mean Velocity Profiles, Downstrean of the Cylinder

Slot, B.L.C. Apparatus (Normal Probe)

$C_{\mu c}=0.2 ; C_{\mu w} \doteq 0.11 ; U_{\infty}=21 \mathrm{~ms}^{-i}$

40. . Spanwise Mean. Velocity Profiles, Downstrean of the Cylinder

Slot, B.L.C. Apparatus (Normal Probe)

$C_{\mu c}=0.22 ; C_{\mu \omega}=0.11 ; U_{\infty}=21 \mathrm{~ms}^{-1}$

41. Mean Velocity Profiles, Downstrean of the Cylinder Slot

B.L.C. Apparatus (Normal Probe)

$C_{\mu_{e}}=0.1 ; C_{\mu w}=0.11 ; U_{\infty}=21 \mathrm{~ms}^{-1}$ 
42. Mean Velocity Profiles, Downstrean of the Cylinder Slot

B.L.C. Apparatus (Normal Probe)

$\dot{C_{\mu c}}=0.2 ; C_{\mu w}=0.11 ; U_{\infty}=21 \mathrm{~ms}^{-1}$

43. Mean Velocity Profiles, Downstrean of the Cylinder Slot

B.L.C. Apparatus (Normal Probe)

$C_{\mu c}=0.22 ; C_{\mu w}=0.11 ; U_{\infty}=21 \mathrm{~ms}^{-1}$

44. Mean Velocity Profiles for Different Values of $C_{\mu \omega}$

(B.L.C. Apparatus)

45. Longitudinal Turbulence Profiles Downstream of the Cylinder slot, B.L.C. Apparatus (normal pröobe)

$C_{\mu c}=0.1, C_{\mu w}=0.11$

46. - Longitudinal Turbulence Profiles Downstream of the Cylinder slot, B.L.C. Apparatus (normal probe)

$C_{\mu c}=0.2, C_{\mu w}=0.11$

47. Longitudinal Turbulence Profiles Downstream of the Cylinder slot, B.L.C. Apparatus (normal probe)

$C_{\mu c}=0.22, C_{\mu w}=0.11$

48. Longitudinal Turbulence Profiles for Different Values of $C_{\mu w}$ (B.L.C. Apparatus)

49. Normal Stress Profiles $\left(\sqrt{\overline{v^{12}}} / U_{m}\right)$, Downstream of the Cylinder Slot, B.L.C. Apparatus (slanted probe) ${ }_{.} C_{\mu c}=0.1, C_{\mu w}=0.11$

.50. Normal Stress Profiles $\left(\sqrt{v^{12}} / U_{m}\right)$, Downstream of the Cylinder Slot, B.L.C. Apparatus (slanted probe) $C_{\mu c}=0.2, C_{\mu w}=0.11$

51. Normal Stress Profiles $\left(\sqrt{\varphi^{12}} / U_{m}\right)$, Downstream of the Cylinder Slot, B.L.C. Apparatus (slanted plobe) $C_{\mu c} \doteq 0.22, C_{\mu w}=0.11$,

52. Normal Stress Profiles, Downstream of the Cylinder Slot, B.L.C. Apparatus (slanted probe) $\theta=32^{\circ}, C_{\mu c}=0.1, C_{\mu w}=0.11$

53. Normal Stress Profiles, Downstream of the Cylinder Slot, B.L.C. Apparatus (slanted probe) $\theta=42^{\circ}, C_{\mu c}=0.1, C_{\mu w}=0.11$

54. Normal Stress Profiles, Downstream of the Cylinder Slot, B.L.C. Apparatus (slanted probe) $\theta=42^{\circ}, C_{\mu c}=0.2, C_{\mu w}=0.11$

55. Shear Stress Profiles, Downstream of the Cylinder Slot, B.L.C. Apparatus (slanted probe) $C_{\mu e}=0.1, C_{\mu w}=0.11$ 56. . Shear Stress Profiles, Downistream of thẹ Cylinder Slot, : 
if Shear Stress Profiles, Downstream of the Cylinder Slot, B.L.C. Apparatus (slanted probe) $C_{\mu c}=0.2, C_{\mu w}=0.11$

58. Shear Stress Profiles, Downstream of the Cylinder Slot, B.L.C. Apparatus (slänted probe) $C_{\mu c}=0.2, C_{\mu w}=0.11$

59. Shear Stress Profiles, Downstream of the Cylinder Slot, B.L.C. Apparatus (slanted probe) $C_{\mu c}=0.22, C_{\mu w}=0.11$

6) Shear Stress Profiles, Downstream of the Cylinder Slot, B.L.C. Apparatus (slanted probe) $C_{\mu c}=0.22, C_{\mu w}=0.11$

61 Shear Stress Profiles, Downstream of the Cylinder Slot, B.L.C. Apparatus (slanted probe) $\theta=32^{\circ}, C_{\mu c}=0.1, C_{\mu w}=0.11$

62. Shear Stress Profiles, Downstream of the Cylinder Slot, B.L.C. Apparatus (slanted probe) $\theta=42^{\circ}, C_{\mu c}=0.1, C_{\mu \omega}=0.11$

63. Shear Stress Profiles, Downstream of the Cylinder Slot, B.L.C. Apparatus (slanted probe) $\theta=42^{\circ}, C_{\mu c}=0.2, C_{\mu w}=0.11$

64. Shear Stress Profiles, $\overline{u^{\prime} v^{\prime}} / U_{m}^{2}$ 
List of Tables

Table

C.1 - Z Multipliers (Appendix C)

$G \quad$ - Tabulated Results (Appendix G)

6

1 


\section{Nomenclature}

A

$B$

$\dot{C}$

$C_{\mu c}$

$C_{\mu w}$

$C_{P}$

$d$

$D$

$\sqrt{e^{2}}$

$\sqrt{\overline{e^{2}}}$

E

$\bar{E}_{l i n}$

4

$\bar{E}_{b r}$

$\bar{E}_{\text {。 }}$

$E$

$\bar{E}_{t r}$

$h$

3

$k$

in

if

$P$

$P_{C 1}$

PC2

$P_{j}$.

$P_{0}$

$P_{t}$
- $\quad y$ intercept of hot-wire calibration curve.

- slope of hot-wire calibration curve.

- $\quad y$ intercept of hot-wire calibration curve.

- cylinder wall-jet momentum coefficient.

- plane wall-jet momentum coefficient (B.L.C. apparatus).

- $\quad$ static pressure coefficient, $\left(P-P_{\infty} / \frac{1}{2} \rho_{\infty} U_{\infty}{ }^{2}\right) ; P_{\infty}=$ atm.

- orifice diameter of flowmeter (Equa. 3.11),

- also cylinder diameter.

- pipe diameter of orifice plate flowmeter (Equa. 3.11).

- linearized RMS value corresponding to $\sqrt{\overline{e^{2}} b_{r}}$, (Equa. 3.5).

- RMS of the AC component of bridge output voltage of hot-wire signal.

- instantaneous voltage.

- linearized voltage corresponding to $\sqrt{\bar{e}_{b r}}$, time mean value (Equa. 3.3).

- bridge output voltage of hot-wire anemometer, time mean value.

- - bridge output voltage at zero flow.

- velocity of approach factor orifice plate flowmeter (Equa. 3.11).

- pressure transducer time mean output voltage.

- pressure head across the orifice, (Equa. 3.11).

- pitch factor of hot-wire probe.

- yaw factor of hot-wire probe, (Equa 3.10).

- mass flow rate per unit span, (Equa 3.13).

- mass flow rate $(l b m / h r)$, (Equa 3.11). .

- static pressure.

- static pressure at the upstream end of tunnel contraction

- static pressure at the downstream end of tunnel contraction

- static pressure at the cylinger wall-jet exit.

- plenưm pressure.

- total pressure at point of maximum velocity. 
$\Delta P$

2

$P_{\infty}$

$Q$

$R_{0}$

$S$

$S^{*}$

$t$

$\bar{t}$

$\Delta \dot{T}$

$T_{j}$

$U_{\infty}$

$U$

-

$U_{\text {eff }}$

$U_{B}$

$U_{N}$

$U_{T}$

$u_{e}$

$u, v, w^{\prime}$

- pressure drop between the chamber and the calibration point.

- static pressure of freestream.

- resultant flow velocity.

- cold wire resistance at reference temperature.

- slope of linearized calibration curve of ho:-wire pribe. slanted probe.

- slope of linearized calibration curve of hot-wire prolun, normal probe.

- jet slot thickness, (plane still air wall-jet apparatus).

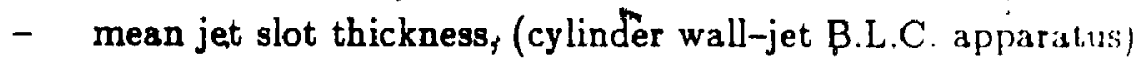

- temperature change in flow field.

- temperture of the jet air.

- tunnel freestream velocity.

- mean velocity.

- effective cooling velocity.

- sensor based velocity bi-normal to wire.

- sensor based velocity normal to wire.

: sensor based velocity tangential to wire. .

- external flow velocity.

- time mean velocity components in $x, y, z$ directions.

$\dot{u}^{\prime}, v^{\prime}, w^{\prime}$. - fluctuating velocity components in $x, y, z$ directions.

$\overline{u^{h} v^{\prime}}, \overline{u^{\prime} w^{\prime}}, \overline{v^{\prime} w^{\prime}} . \quad-\quad$ Reynolds' shear stress components.

$V_{n} \quad-\quad$ velocity normal to the porous end plates.

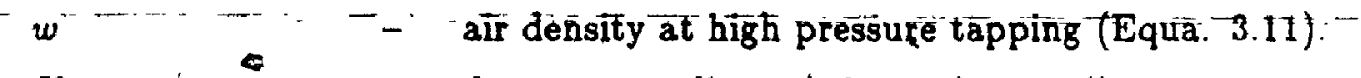

$X$

- downstream distance from plane wall-jet slot

(B.L.C. apparatus), :

- also downstrea distance from plane wall-jet slot, (still air wall-jet apparatus).

$Y \quad-\quad$ distance from cylinder surface. 
- correction multiplier for orifice plate flowmeter

(Equa. 3.11),

or

- spanwise distance from the centreline of cylinder and plane wall-jets (B.L.C. apparatus).

. Greek Symbols

$\alpha$. $\quad$ hot-wire probe angle (the complementary angle to

the angle between the probe stem and the wire sensor

(Figure 19);

- also pitch angle (Figure 16).

$-5, \xi, \eta$

- Gxed coordinate system of hot-wire probe, (Figure 19).

$\theta$

- distance from the cylinder slot exit, specifies measuring station.

$\rho \quad$ - density of air during calibration.

$\rho_{\infty} \quad$. $\quad-$ density of air at freestream conditions.

$\rho_{j} \quad$ is density of jet air.

$\psi \quad$ - orientation angle of hot-wire sensor (i.e. angle through which the hot-wire probe in rotated about its stem, Figure 19 ). 


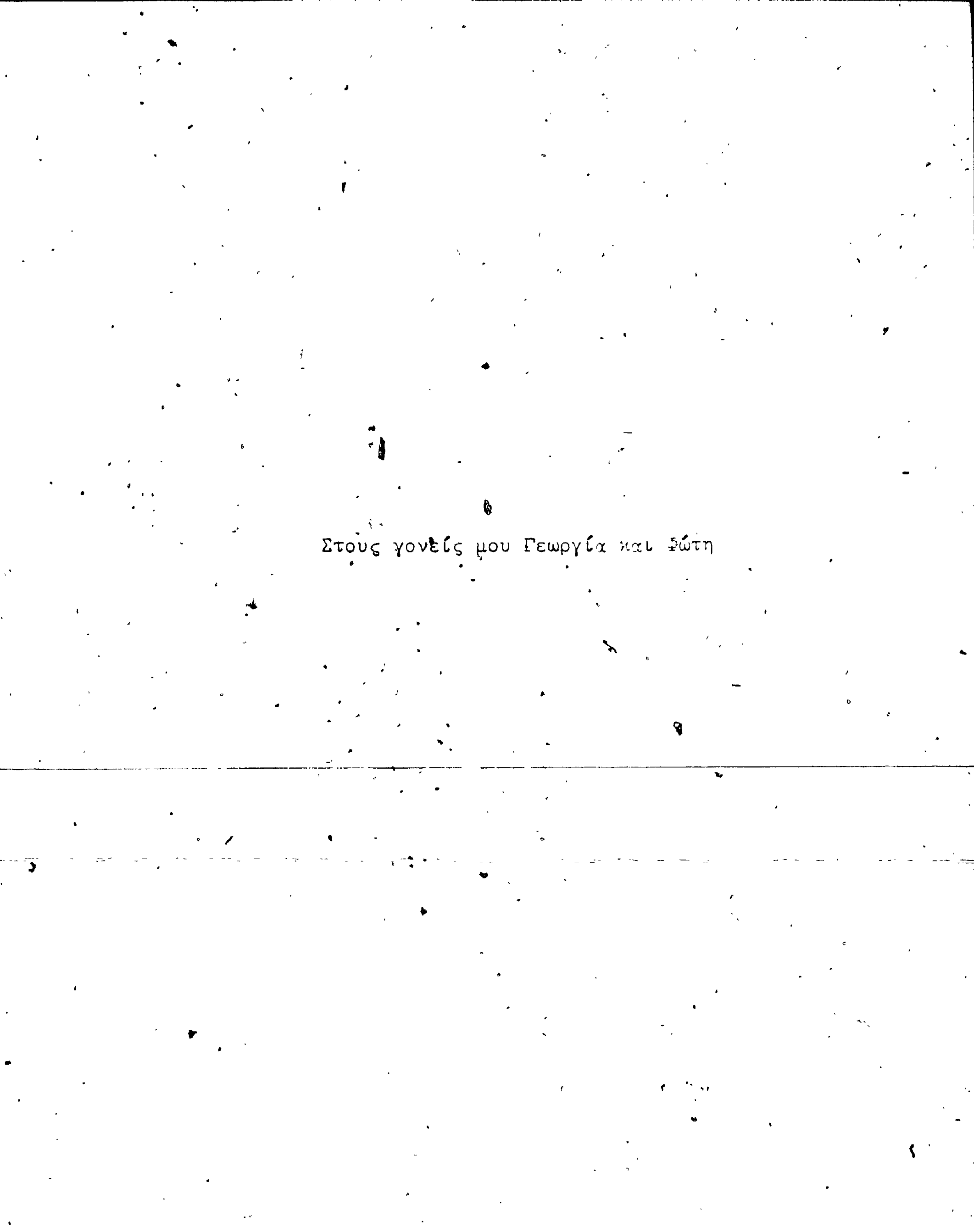




\section{Chapter 1 Introduction}

\subsection{Background}

1

When flows encounter a region with unfavourable pressure gradient, that is if the pressure increases downstream, the retarded fluid particles cannot penetrate toofar into. the region of increased pressure owing to their low kinetic energy. Strong adverse pressure gradient effects overcome momentum and shear stress effects which tend to keep the fluid from moving forward. The How 'can no longer, remain attached to the solid boundaries and . it separates from them. Further downstream of the point of flow separation, the adverse gradient effects tend to push the fluid near the boundaries backwards thus causing a reverse How.

Flow separation has detrimental effects on the aerodynamic characteristics of the device involved because it is associated "with large pressure drag and. as a result, loss of lift. Boundary layer control (B.L.C.) is a means of preventing or delaying flow separation. The B.L.C. concept is particularly attractive to high lifting surfaces, such as aircraft wings and it has been extensively studied as at meáns of minimizing drar at cruising speeds, of maximizing lift at landing and takeoff and minimizing unsteady flow effects in general $[41]$.

High performance fighter aircraft, VSTOL aircraft, carrier based aircraft and helicopters are the most prominent contenders for B.L.C. applications. The two most promising methods of B.L.C. include suction and blowing.

Boundary layer control by suction is the process in which low kinetic energy fluid is removed from the boundary layer by applying suction thróng series of slots or perforations on the solid boundaries. The resulting fuller,yelocity profile shape is such that the shear stresses near the wall are greater and the flow canjadvance further in the region of increased. pressure.

The boundary-layer control by blowing concept relies on the re-energization of the low kinetic energy fluid near the wall for, delaying flow separation and decreasing drag. When high energy fluid is injected tangentially into the boundary. layer, it increases its kinetic energy and the resulting robust velocity profile shape is such that it can advance further into the increased pressure region. Tangential injection of high energy fluid at the rounded 
trailing edge of airfoils is a promising way of achieving circulation control. The injected, fluid remains attached to the rounded surface by virtue of the Coanda effect as illustrated in Figure 2. Air for blowing can be supplied from the gas turbine engine compressor bleol The delay of flow separation increases the circulation around the airfoil and as a result its. . lift. By varying the amount of blowhy, it is possibfle'to achieve circulation control (C C.) 1 , around the airfoil. The flow around a circulation controlled airfoil is illustrated in tigure..." 1.1

- Due to the lack of a sharp trailing edge and the associated Kutta condition, circtulat.." control by tangential injection may be achieved at low blowing rates. High lift coeflicients ard low effective drag make this scheme very attractive, br fixed-wing STOL atrcraft and . rotorcraft:

This study is concentrated on the understanding of the.flowfield similar to that observed downstream of the injection slot of a rounded trailin edge of a circulation controlled airfoil.

1.2 Nature of Flow $: \ldots$

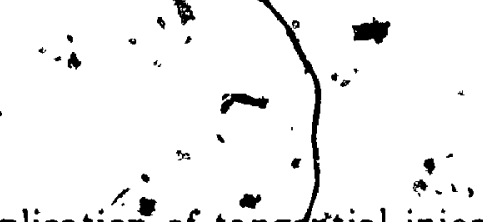

The flowfield associated with application of tang ritial injection is relatively complex because it involves the mixing of a tusulent wall-jof yith a turbulent boundary layer in a strong adverse pressure gradient ơn a highly cưrveq surfạce.

- Figure 3 shows a typical wall-jet over a Curved sŭrface in a moving stream, The velocity profiles downstream of the slot are usually not symmetrical and the flow is not self preserving. The possible behavior of wall-jets in a ftecstream on in adverse pressure gradient has been described by Dvora ${ }^{[17]}$, see Figure 4.

The development of the flow may proceed in a number of different ways; namely;

a). The velocity minimum is completely entrained by the wall-jet.

b) Both the velocity minimum and the velocity maximum persist.

c) Both the velocity minimum and the velocity maximum disappear. '

, $\quad \because \quad$ Flow separation cat take place by either of two distinct mechanisms:

$\therefore$ The flow can separate from the solid boundary.

- The velocity minimum, if it persists; can be deepened by a sufficiently strong adverse pressure gradient until reverse flow occurs and the external flow separates from the 
wall-jet flow.

The two major factors affecting the flow development are severity of the adverse pressure gradient and the momentum defect of the upstream boundary-layer as compared with the jet momentum.

\subsection{Previous Research}

Within the broad definition of a turbulent wall-jet, a wide range of flows and phenomena have been researched. In this section, only a small number of wall-jet flows that have similarities with the flowfield described in Section 1.2 will be presented.

Kruka and Eskinazi $\{40\}$ have carried out noteworthy research on wall-jets in a moving stream. Irwin ${ }^{[32]}$ in later work has reported measurements including mean velocity, turbulence stresses, triple and quadruple velocity cortelations, intermittency and spectra of the longitudinal turbulence intensity. Launder and Rodi ${ }^{[42]}$ found Irwin's data suitable for testing numerical methods. Erian ${ }^{[20]}$ has investigated the influence of pressure gradient on turbulent flows with asymmetric mean velocity, using a plane wall-jet in a frestream.

Wilson and Goldstein ${ }^{[53]}$ have investigated surface curvature effects on the development of a two-dimensional jet. The wall-jet in their experiments, developed around the convex surface of a circular cylinder in still surroundings. Surface curvature effects on flow development have also been studied by Guitton and Newman ${ }^{[29]}$, Giles et al. ${ }^{[26]}$ with wall-jet flows over logarithmic spiral surfaces in still air.

Considerable work on wall-jets on plane and curved surfaces in still surroundings or in a free stream has been carried out at McGill University. Launder and Rod ${ }^{42]}$ provide. fan extensive review of work on turbulent wall-jets and evaluation of experimental data for use in computational schemes.

Attempts for developing calculation methods for prediction of the dev :lopment of walljets in arbitrary pressure gradients have been made by Kind ${ }^{[37]}$ and $D$ vorak $k^{[17 !}$ among others. Kind ${ }^{37]}$ has compared his calculation method with the experimental data of McGahan [44], Kind ${ }^{(57)}$ and Fekete ${ }^{[23]}$. These authors could only do limited tests on their methods due to limited amount and range of experimental data suitable for use as test cases. Later work of Kind et al. ${ }^{\mid 38}$ provided measurements of flows with tangential injection and comparison with two prediction methods. This work involved profiles in eighteen different two-dimensional flows over plane and curved surfaces with a variety of adverse pressure gradients. The data 
were Compared with an integral method of Kind ${ }^{37]}$ which used four paraneters to describe the velocity profiles, and a finite difference method of Dvoraki17!, using an eddy viscosity: model to establish shear stress in the flow. Both calculation schemes showed weaknesses: in predicting the flow near the separation points. The authors ${ }^{[38]}$ concluded that additional flow field data was desirable to further assess capabilities of prediction methods and 1 " guide efforts to improve them. In complex flows of this type, agreement within about $417^{\prime \prime}$ of velocity is considered successful ${ }^{[38]}$.

The present work attempts to provide measurements of mean velocity and turtsulenu. in a flow field very similar to that described by Kind et al $\left.\right|^{|38|}$. These measurements ibre intended to assist in the testing of calculation methods.

\subsection{Purpose of the Current Study}

The main purpose of the present study is to provide suitable data for testing of prediction methods in flows with tangential injection. The progression from planning to completion of this task involved the following major steps;

i) Familiarization with the principles of hot-wire anemometry and implementation of a data acquisition system for gathering data.

ii) Derivation of hot-wire equations and experimentation to the equations.

$\checkmark$

iii) Measurements of mean velocity and turbulence in a plane wall-jet and comparison of results with that of other experimentors.

iv) Modification to the boundary layer control apparatus.

v) Experimentation on the boundary laye control apparatus. 


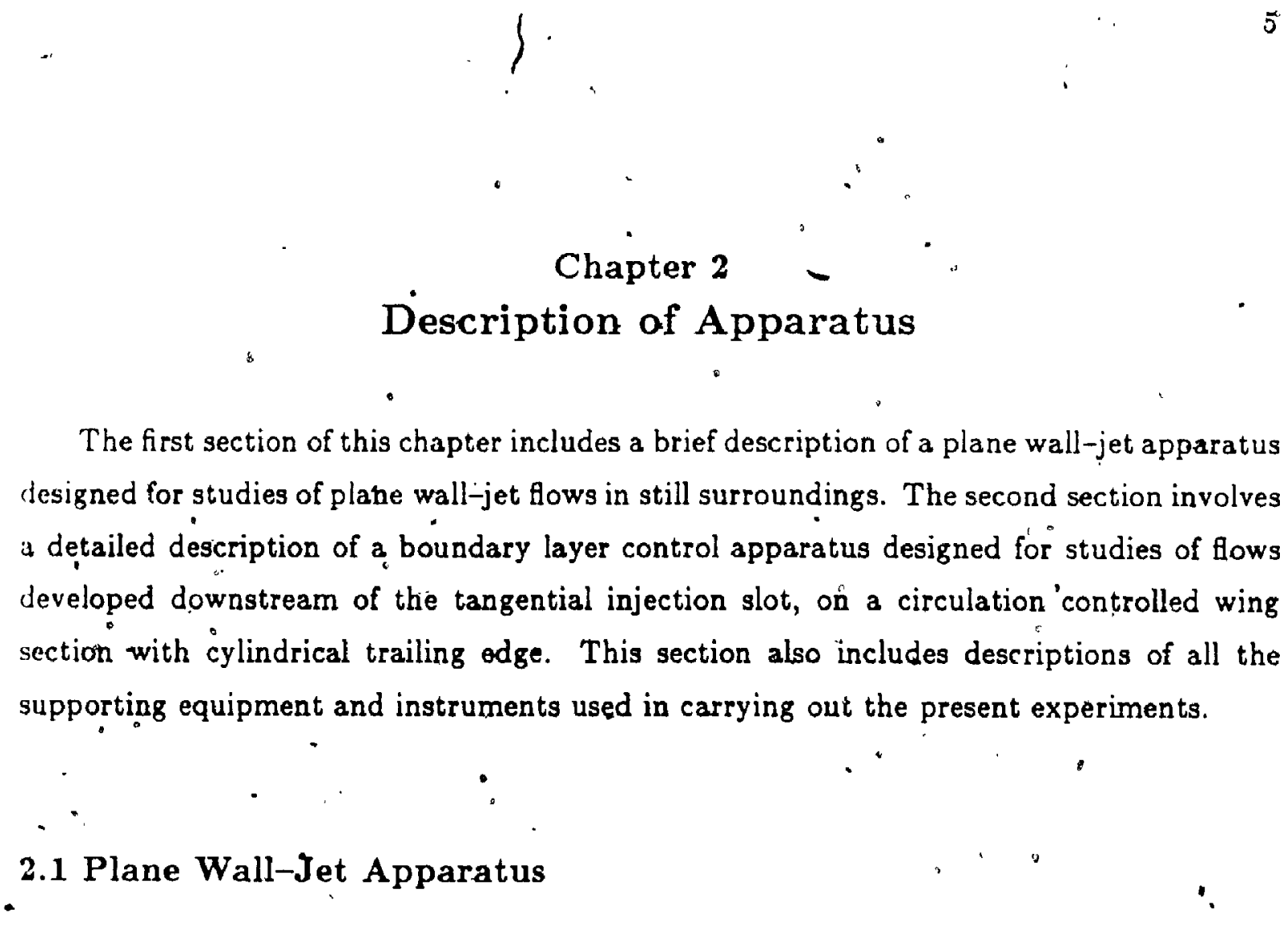

A plane wall-jet apparatus, which was already in existence, was used in the first stages of this study. A photograph of this apparatus is shown in Figure 6.

Air from a plenum chamber discharged tangentially to a $38.1 \mathrm{~cm}$ long and $30.5 \mathrm{~cm}$ wide polished steel plate, through a slot of thickness $t$ equal to $1.587 \mathrm{~mm}$. The slot thickness varied less than $\pm 0.12 \mathrm{~mm}$ across the entire $30.5 \mathrm{~cm}$ span. The chamber was continuousty supplied with air from both sides by a centrifugal blower. Two $58.4 \mathrm{~cm} \times 20.3 \mathrm{~cm}$ side plexiglass plates assist in maintaining two-dimensional flow"conditions downstream of the -

An aluminiumbracket clamped between the side plates provides support to the traversing mechanism which was driven by a stepper motor. In this arrangement, the transverse gear moved a guide tube along an axis perpendicularly to the flow surface of the apparatus. A drilled aluminium block allowed probes to be supported on the guide tubes near the flow - surface, to be aligned with the flow and to be rolled at any desired angle. Figure 11 shows the traversing gear fitted to the wall-jet apparatus.

Suthanthiran ${ }^{[50]}$ provides a more detailed description of the wall-jet apparatus together with a number of checks of flow two-dimensionality. His flow visualization studies and mean velocity measurements showed that the flow is two-dimensional, except near the side walls. 


\subsection{Boundary Layer Control Apparatus}

The boundary layer control (B.L.C.) apparatus used in the present study is a mul. ified version of that used in previous studies of bounfary layer control with tangenthit injection ${ }^{[38]}$. A schematic diagram of this "modified". B.L.C. apparatus is shown in rirutu" 8 , and its components will be presented in following sub-sections.

\subsubsection{Wind Tunnel}

The wind tunnel used for this study is a closed circuit low-speed facility lociatid ... Carleton University. A single stage fan powered by a $50 \mathrm{hp}$ electric motor provided the. drive of the wind tunnel. The maximum wind speed at the tunnel test section way $1.50 . \mathrm{m}, \mathrm{h}$ at approximately $1500 \mathrm{rpm}$ fan speed. The pitch of the fan blades was set to $30^{\circ} \mathrm{A} \quad \mathrm{I}_{\mathrm{r}} \mathrm{l}$ contraction ratio with five screens in the settling chamber gave a longitudinal turbulent ' level at the test section of approximately $0.15 \%[27]$.

The test section freestream velocity was computed according to the following wind tunnel calibration relationship;

$$
\frac{P_{C 1}-P_{C 2}}{0.91}=\frac{1}{2} \rho_{\infty} U_{\infty}^{2}
$$

where $P_{C 1}$ and $P_{C 2}$ are the static pressures at the upstream and downstream ends of the tunnel contraction respectively. The pressure differential was monitored by an inclined (1:2 slope) single tube micro-manometer filled with oil of a specific gravity of 0.826 and sensitivity of $\pm 0.5 \mathrm{~mm}$. Adjustments of the tuanel speed were possible by varying the spered of the fan. Previous calibration measurements showed that the wind speed across the test section was approximately constant $( \pm 3 \%)$.

\subsubsection{Test Section,}

All the components of the B.L.C. apparatus were enclosed in a 20 in by 30 in by $6 / \mathrm{ft}$ long removable test section that could be fitted in the wind tunnel circuit. The test section is made from wood with plexiglass front and back windows.

\subsubsection{Plane Wall and Cylinder Wall-Jets}

The back wall of the test section was fitted with a blowing slot as shown in Figure 8, mainly for purposes of an earlier experiment. The vertical stot has a mean exit thickness of 

velocity profiles (Kind et al $\left.{ }^{\{38}\right)$. Although these measurements provided data for fluw cases. where fluid from a wall-jet mixes with an upstream boundary layer in a strong adverst' pressure gradient, the boundary layer approaching the blowing slot was very thin, this is not typical of many applications where B.L.C. is used.

It was later understood that a forebody extending upstream of the circular cylunder would produce a thicker boundary layer at the slot, thus making the flow conditums mur". realistic. W. Kelly ${ }^{[34]}$ worked towards the development of a forebody shapd for the "Sistul" bqundary layer control apparatus, using a computer solution for incompressible 1 w airfoils of arbitrary section. His work produced a forebody shape that satisfied the de. considerations of favourable or modẹrately adverse pressure gradients near the s!ou

This shape was also adopted in constructing a forebody for the present stiudy. The forebody consisted of a "Lexan" skin, ( $\frac{1}{16}$ in thick) screwed to 4 ( $\frac{1}{4}$ in thick) plexiglass ribs. One row of static pressure taps was fitted at mid-span around the forebody. The forebody is shown in Figures 8,11 and 12. Two bolts, one on each end near the nose of the forebody, running through the upper and lower ends of the test section kept the forebody in place.

\subsubsection{Traversing Gear}

The traversing gear used in the plane wall-jet apparatus has already been mentioned in Section 2.1. A stepper motor, DISA type 52C01, transfers movement to a toothed givide tube via a 10-teeth pinion gear. With the stepper motor set at a 10:1 gear ratio, the probe movement was $0.025 \mathrm{~mm} / \mathrm{step}$. The selection of $10: 1$ gear ratio minimizes gear slip and enables traversing of very thin layers. The stepper motor was controlled by a sweep drive unit (DISA 52B01). The sweep drive unit enables remote control and offers a chotce of both forward and backward sweep, variable sweep time, together with upper and lower stop capabilities. It was quickly realized that traversing could be controlled by the ADACS system if the "stop" connector of the sweep drive unit was properly connected to a system actuation relay. A considerable time was spent in perfecting the control of the traversing, māking it as flexible as possible. The software developed for this function was incorporated in the ADACS computer programs, U\&RMS and M\&RMS described in Appendix D.-

Although the traversing gear described earlier was suitable for measurements on the still air wall-jet apparatus, it had to be modified considerably for measurements on the boundary layer control apparatus.

A new traversing mechanism was designed for traversing a hot-wire probe across the 
tritndary layer developed downstream of the slot exit of the boundary layer control apparat.us. This mechanism is shown in Figure 10 and the photograph of Figure 11. In this arrangement, the stepper motor was placed inside of the cylinder jet away from the end wall porous plates. Two steel supports fastened inside the tunnel supported both the stepper motor and the probe holder. The probe can be set at an 9 desired roll angle. A protractor allows measurement of the roll angle. Two rubber cushions, $3 \mathrm{~mm}$ thick, were placed on both ands of the steel supports to damp vibrations transmitted through the tunncl structure.

A simple kinematic analysis of the stepper motor, probe holder arrangement of the traversing gear is carried out in Appendix A. Thus, given the guide tube displacement It was possible to determine the corresponding probe sensor movement. This analysis was easily incorporated in the data acquisition and control software developed for these experiments, (see Appendix A).

\subsubsection{Orifice-Plate Flowmeters}

Orifice-plate flowmeters were used for mass flow measurements in the cylinder walljet and the suction system. The flowmeters were designed in accordance with British Standard $1042^{[10]}$. The orifice diameter, $d$, was $1 \frac{3}{16} i \pi$. Two pressure taps, one at a distance, $D$, upstream of the orifice and one at a distance, $\frac{D}{2}$, downstream of the orifice enabled measurements of pressure changes across the orifice. A dial thermometer fitted. downstream of the orifice monitored the temperatures of the flowing air. Figure 9 shows the orifice-plate flowmeters in place. Mass flow measurement procedures will be presented in Chapter 3.

\subsubsection{Manometers and Pressure Transducer}

A multitube manometer board consisting of fifty tubes, in the vertical position, was used to measure static pressure distributions around the cylinder and forebody, and the static pressure inside both jet plenum chambers. During any particular run, all press yre lines leading to the board could be clamped off, thus "freezing". the pressure distributidn

shape for inspection or for taking data. The manometer board could be read to an accuracy of approximately \pm 0.05 in of water. Another three multitube manometers consisting of twenty tubes in the vertical position, were used to measure static pressure distributions along the back wall of the test section. These manometers could be read to an accuracy of appróximately \pm 0.05 in of water. 


\subsubsection{Blowers}

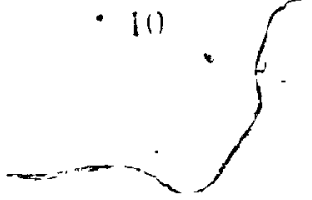

The cylinder plenum chamber was supplied by a unit consisting of two $1 \mathrm{hp}$ varuum cleaner units arranged in parallel and fitted in a wooden casing. Two screens, onte th the suction side and the other at the blowing side were installed in an attempt to filter the ur before it entered the plenum. The wall blowing slot was supplied with air by a he'avy diut: Dustbane blower unit, with a flow rate of 92 cubic feet/minute at full speed. Thi; unit was also equipped with a $656 s q$. in dry air filter. Each porous end-plate was ronner. i to the suction side of two similar Dustbane blower units, connected in parallet. "The print and hence the flow rate of the blowers was controlled by single variacs cach connecteri 1 , different circuits of the laboratory. A schematic diagram of the blowing air and suctum system is shown in Figure 9.

\subsubsection{Hot-Wire Anemometer}

A constant temperature anemomete Thermo Systems Inc. Model 1053 A, was used to measure mean and fluctuating flow velocities in the present study. This type of anemometur utilizes a feedback controlled wheatstone bridge circuit for maintaining the sensor at constant temperature. The typical operating range of the sensor is $150^{\circ} \mathrm{C}$ to $260^{\circ} \mathrm{C}$. The three arms of the wheatstone bridge have a constant resistance and the fourth arm is the sensor Any variation in the sensor resistance due to changes in fluid velocity over the sensor is corrected by automatic re-adjustment of the current through the sensor. The output of the anemometer is the voltage required to drive the necessary current through the sinniss to maintain its temperature at constang value.

The anemometer unit is also equipped with a TSI, model 1052, linearizer, for linearizing the bridge voltage versus flow velocity relationship.

The selection of the sensor operating temperature is limited by the mechanical properties of the sensor material. High operating temperatures.mprove sensor response to flow velocity fluctuations and minimizes temperature effects (Bidshaw $\left.{ }^{6}\right)$. The two types of hot-wire probes used for the present measurements were DISA single miniature probes, the $55 \mathrm{P} 11$ normal probe and the $55 \mathrm{P} 1245^{\circ}$ slant probe. The probe sensor is a tungsten wire of $5 \mu \mathrm{m}$ diameter and $1.25 \mathrm{~mm}$ in length, welded to the tips of the probe prongs. These probes could operate at a maximum temperature of $300^{\circ} \mathrm{C}$, allowing a maximum overherat ratio of about 1.9. This value was used throughout the present experiments. Additionial instruments used in conjunction with the anemometer unit include a Tektronix, type $221 ?$ 



\subsection{Automatic Data Acquisition and Control Systems (ADACS)}

The Hewlett-Packard, model $3954 \mathrm{~A}$, is a computer based system with precision ineasurement and control capabilities. This system was utilized in the present study for gathering data from a hot-wire probe and for control of probe traversing. The system includes a specialized software support package that is designed to use the system instruments to achieve specific operations and functions. The system's components are descrilied win. ;ately.

\section{System Computer.}

The system computer is the HP-85A desktop computer. The computer is interfacen with the system control unit via the 82937 A HP-IB I/O (input/output) cord. The Hewlett Packard (HP-35A) computer has a total memory of $32 \mathrm{~K}$ and it can be programmed using a.version of the BASIC programming language. Its features include a cathode ray tube display, an internal printer, a tape drive auto-stárt and error trappinğ.

\section{Digital Voltmeteds}

The ADACS system voltmeters are the Hewlett-Packard HP-3456A and the HP$3437 \mathrm{~A}$. The HP-3456A digital voltmeter is a $3 \frac{1}{2}$ to $6 \frac{1}{2}$ digits, integrating voltmeter, with $100 n V$ sensitivity. Its capabilities include DC and RMS $\mathrm{AC}$ voltage measurements in the range of 0 to $100 \mathrm{~V}$. It also has. selectable integrating times from 0.01 to 100 . power line cycles, and it eliminates noise by an analog input filter and digital averaging. Its $\mathrm{Tead} / \mathrm{write}$ memory can hold up to 350 readings with a specified delay between readings. The $\mathrm{H} \dot{P}-$ $3456 \mathrm{~A}$ voltmeter can be programmed and triggered through the front panel (cxternally), internally or through software.

\section{Data Acquisition/Contrql Unit}

The 3497 Data Acquisition/Control Unit combines precision measurement and cortrol capabilities. Its standard features include a built in real time clock, front panel display/controls and HP-IB interfacing. The control unit is equipped with twenty channels that can be used for data acquisition and sixteen relay actuator channels." In the present experiments, two channels were used for data acquisition and one channel for control $A$ schematie diagram of the ADACS system connected to tha hot-wire anemometer unit is shown in Figure 13. 


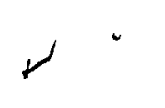

r.

\section{Chapter 3 \\ Experimental Procedures}

\subsection{Experimentation on the Plane Wall-Jet Apparatus (Still Surroundings)}

The Plane wall-jet apparatus described in Section 2.1 and shown in Figure 6, was used in the earlier stages of this study for the following tasks;

(i) Familiarization with hot-wire measuring techniques.

(ii) Development and testing of ADACS computer programs....,

(iii) Evaluation of hot-wire signal analysis.

(iv) Comparison of results to those of previous investigators.

A short description of the experimental procedures for carrying out the aforementioned tasks will be described in the following subsections.

\subsubsection{Familiarization with Hot-Wire Measuring Techniques}

Familiarization experiments involved calibration of hot-wire probes and linearization of the anemometer output signals using the TSI 1052 electronic signal linearizer. The linearizer coefficients were calculated and setaccording to the procedures outlined in the TSI linearizer manual. A series of experiments was carried out with and without the electronic linearizer, and results were compared. The calibration and setting of the linearizer coefficients had to be repeated about every two hours due to the linearizer output signal drifting with time. . The use of the electronic signal linearizer was a long and tedious process.

It was realized that an easier and more accurate method had to be employed for linearizing the anemometer output signal. The TSI FORTRAN version, linearization program was made available by, Yowakim ${ }^{[55}$. The linearizer coefficients calculated by the program were set on the linearizer. A simple test of the program was performed by comparing mean velocity measurements taken with and without the linearizer. A listing and a sample output program, that can be used as a future reference is included in Appendix B. 


\subsubsection{Development and Testing of ADACS Computer Programs}

The HP-305A data acquisition'system was used for sampling of both the time-me.11 $\left(\bar{E}_{b r}\right)$ and the RMS $\left(\sqrt{e_{b r}^{2}}\right)$ of the output hot-wire output signal. The ADACS progrin developed in this study used system subprograms as a basis for sampling the hot wre" signals. The function of these subprograms was to program and trigger the 111 1.3.4ti vill meter. Thus, the system subroutine" "Avdcr" and the "Acv" were used for meatirmin. of $\bar{E}_{b r}$ and $\sqrt{\bar{e}_{b r}}$ respectively. A set of data taken in the wall-jet at $x / t=4,34$ howr. a considerable scatter when the subroutines were first tried. This lead to a sertes .t... periments in the wall-jet apparatus with the objective of obtaining repeatable hot wir:" measurements in a short period of time, "optimum sampling rate". In these experimetit. the hot-wire probe was kept apptoximately at the point of the maximum shear stress wi the boundary layer at $x / t=93.3$, (i.e. at that point the scatter of the data was maximum)

Experimentation showed that by increasing the number of power line cycle (PLC) in. tegration times of the HP3456 voltmeter, better results couldxbe obtained. Thus, the voltmeter was programmed for maximum PLC integration time 100. Large PLC integration time increases the accuracy, repeatability and reduces the noise. At 100.PLC integration time, sampling the noise reaction was $75 d B$ and the error in the measurements was approximately $0.0015 \%$. Thus, for a complete sampling of $\bar{E}_{b r}$ and $\sqrt{\overline{e^{2}}{ }_{b r}}$ at 100 PLC integration time and, $60 \mathrm{~Hz}$ power line frequency, it took the voltmeter about $1.7 \mathrm{~s}$. Further experimentation showed that by increasing the number of readings/trigger, the results were more repeatable. Thus the voltmeter was programmed to average 999 readings/trigger. For better accuracy, the voltmeter was triggered thirty times for sampling $\vec{E}_{b r}$ and thirty times for sampling $\sqrt{\overline{e^{2}}}$. The total time spent at the "optimum sampling rate" was 102 s for each point. The errors involved in sampling both $\bar{E}_{b r}$ and $\sqrt{\bar{e}^{2}}$ were less than $0.5 \%$ at this sampling rate.

The number of times that the voltmeter is triggered can be adjusted through the software. The programs ceveloped to sample the hot-wire signals and control the traversing in wall-jet apparatus experiments are earlier versions of the program "C\&RMS" and "M\&RMS" shown in Appendix D.

The evaluation of the hot-wire signal analysis will follow in Section 4.3. The nomenclisture and probe orientation during experiments with the plane wall-jet apparatus is shown in Figure 14.' A set of results obtained from the plane wall-jet will be presented and compared 
to results of other investigators.

\subsubsection{Hot-Wirè Calibration and Linearization Methods}

The hot-wire probes were calibrated using the calibration apparătus shown in Figures * 7 and 15 . Before collecting Hata, the settling chamber and the probe support device were leveled and the base of the apparatus was clamped to the working bench. In this arrangement the flow diredtion through the nozzle was kept horizontal; i.e. parallel to the $x$-axis of the cuordinate system shown in Figure 15. The.probe mounting mechanism kept the probe rigidly in the potential core of the jet, $4.3 \mathrm{~cm}$ downstream of the nozzle-exit plane and on the nozzle center line. The probe mounting mechanism was equipped with a protractor system so that the probe could be set at azero pitch and zero yaw angles during calibration. The inside of the nozzle was smoothed to ensure that' structural irregularities did not caused flow separations. The turbulence intensity at the maximum flow speed of $70 \mathrm{~ms}^{-1}$. was approximately $2 \%$. Yowakim ${ }^{[55]}$ and $K$ elly ${ }^{[34]}$ have also found that the flow through the same settling chamber was essentially onédimensional. Intentioríal changes of roll, yaw and pitch angles of the probe provided estimation on the effect of prabe misalignment on . the $\bar{E}_{b r}$ measurements, Less than $1 \%$ change in $\vec{E}_{b r}$ were detected when probe angles were varied to $\pm 2^{\circ}$ at high flow speeds.

Having ensured good flow one-dimensionality and probe alignment, actual data could be collected. In this study, twenty simultaneous measurements of the mean bridge output voltage, $\bar{E}_{b r}$, and the pressure transducer output voltage, $\bar{E}_{t r}$, were taken during calibration. It was believed that a better approximation of the $\bar{E}_{b r}$ versus flow velocity, $U$, relationsinip . would be obtained using a large number of data points especially at the low velocity region. The flow velocity was estimated by the following relation;

$$
\because u=\sqrt{\frac{2 \Delta P}{\rho}}
$$

The differential pressure, $\Delta P$, was measured with a pressure transducer and its output sig-nal, $\bar{E}_{t r}$, was related to $\Delta P$ by a calibration process. The ADACS system was programmed to average a total of 2000 points for both the $\bar{E}_{b r}$ and $\bar{E}_{t r}$ signals at each data set. The simultaneous input of the flow temperature provided a correction to the air density and thus the flow velocity calculation. The calibration procedure took approximately twenty minutes from start to completion. The temperature of the, air increased an average of $4^{\circ} \mathrm{C}$ during this period and temperature effects on hot-wire measurements were not considered. Sufficient time was allowed between readings for establishing steady-state conditions. The 
- relationship between flow velocity and the bridge output voltage is described by king's liaw as;

$$
{\overline{E^{2}}}_{b r}=A+B u^{n}
$$

Values of $n$ :are such as to provice a better fit to the nonlinear relationship; typically $n=0.45,0.5^{*}$.

A direct relationship between the bridge voltage and the flow velocity may be obtainte by either passing $\bar{E}_{b r}$ through an electronic signal linearizer or by computational methods. Although the anemometer unit was equipped with an electronic signal, linearizer (TSI model 1.002), it was prefered to use a, computer program for signal linearization, which would increase the speed of the measurements. The TSI FORTRAN linearization program was converted to the ADACS Basic language version. A listing of the computer program developed for calibration of hot-wire probes "CALIB" is shown in Appendix. B. This program approximates the response of the anemometer bridge output with a fourth degree polynoimial and cónverts $\bar{E}_{b r}$ to linearized voitages, $\bar{E}_{\text {lint }}$, by the following relation;

$$
\vec{E}_{l i n}=S\left(A_{0}+A\left(\bar{E}_{b r}-\bar{E}_{0}\right)+B\left(\bar{E}_{b r}-\bar{E}_{0}\right)^{2}+C\left(\bar{E}_{b r}+\bar{E}_{0}\right)^{3}+{ }_{v} D\left(\bar{E}_{b r}-\bar{E}_{0}\right)^{4}\right)
$$

where $\vec{E}_{\circ}$ and $\vec{E}_{b r}$ are the bridge output at zero and non-zero values, respectively, of the efective cooling velocity, $S$ is the slope of $\bar{E}_{\text {lin }}$ ys $u$ curve and $A_{0}, A, B, C, D$ are the . $\therefore$ coefficients of the polynomial calculated by the computer program. Thus, $\bar{E}_{l, n}$ may be related to the flow velocity as;

$$
\bar{E}_{l i n}=S u
$$

The same linearization procedure was the procedure followed by Yowakim ${ }^{[55\}}$ and Kellyi ${ }^{\mid 34)-:}$ who had-also converted the TSI Fortran linearization program to ADACS Basic language, with excelleirt resülts.

$\because \quad$ The method of computing the linearized RMS signal $\sqrt{e^{2}}$ lin from the measured RMS of the bridge output signal $e_{b r}^{2}$ is outlined in the following; assume that for low turbukence cases, :

$$
\therefore \quad \sqrt{\overline{e^{2}} l i n}=\Delta \bar{E}_{l i n} \text { and } \cdot \sqrt{e^{2} \cdot b r}=\Delta \bar{E}_{b r} \text {. }
$$

or

$$
\sqrt{\overline{e^{2}} l i n}=\frac{\Delta \bar{E}_{l i n}}{\Delta \bar{E}_{b r}} \sqrt{\overline{e^{2}}}
$$


taking the limits would give

$$
\sqrt{\overline{e^{2}} l i n}=\frac{\partial \bar{E}_{l i n}}{\partial \bar{E}_{b r}} \sqrt{\overline{e^{2}} b r}
$$

Differentiation of equation 3.3 determines $\partial \bar{E}_{l i n} / \partial \bar{E}_{b r}$ which if substituted in the above relationship will give the final expression for $\sqrt{\bar{e}^{2} \text { ln }}$ (Yowakim ${ }^{[55]}$ ). Thus;

$$
\begin{aligned}
\sqrt{\sqrt{\bar{e}_{l i n}}=S}\left(A+2 B\left(\overline{E_{b r}}-\bar{E}_{0}\right)+3 C\left(\overline{E_{b r}}-\bar{E}_{0}\right)^{2}\right. \\
\left.+4 D\left(\bar{E}_{b r}-\bar{E}_{0}\right)^{3}\right) \sqrt{\overline{e^{2}} b r}, .
\end{aligned}
$$

As a check on the method, various voltage signals from the anemometer bridge unit were input to the TSI electronic signal linearizer and itsoutputs compared well with the values calculated from equations 3.4 and 3.5 .

\subsubsection{Determination of the Sensitivity Coefficients}

W Studies by Champagne $e^{\prime 3\}}$ and Mojola ${ }^{\mid 45}$ have demonstrated the importance of the yav sensitivity coefficient, $k$, in accounting for the tangential cooling velocity encountered with inclined probes. Jorgensen $[33]$ showed that in turbulent flows the overall directional characteristics of the probe must be composed of,a pitch correction factor, $h$, in addition to the yaw correction factor, $k$.

- Figure 16 shows an arbitrary orientation of a hot-wire probe with respect to the flow direction. Then the effective cooling velocity acting on the sensor is given by Jorgenseni ${ }^{33}$ as;

$$
u_{e f f}^{2}=u_{z}^{2}+h^{2} u_{v}^{2}+h^{2} u_{z}^{2}
$$

where $k$ and $h$ are the yaw and pitch correction factors respectively.

If the pitchangle, $\theta=0$, équation 3.6 becomes;

$$
u_{e f f}^{2}=u_{x}^{2}+k^{2} u_{v}^{2}
$$

or

$$
u_{\theta f f}^{2}(\alpha)=u_{\theta=0}^{2}\left(\cos ^{2} \alpha+k^{2} \sin ^{2} \alpha\right) \text { for } \theta=0
$$

If the yaw o angle, $\alpha=0$, equation 3.6 becomes;

$$
u_{a f f .}^{2}=u_{x}^{2}+h^{2} u_{z}^{2}
$$

or 


$$
\text { - } u_{e f f}^{2}(\theta)=u_{\alpha=0}^{2}\left(\cos ^{2} \theta+h^{2} \sin ^{2} \theta\right) \text { for } \alpha=0
$$

If $u$ is substituted with $u_{e f f}$ in equation 3.4;

$$
E=S u_{e f f}
$$

Substituting equat 3.7 and 3.8 into equation 3.4 it becomes;

$$
\frac{\bar{E}(\alpha)^{2}}{S^{2}}=\frac{\bar{E}(0)^{2}}{S^{2}}\left(\cos ^{2} \alpha+k^{2} \sin ^{2} \alpha\right)
$$

or

$$
\dot{k}=\frac{1}{\sin \alpha}\left(\frac{\bar{E}(\alpha)^{2}}{\bar{E}(0)^{2}}-\cos ^{2} \alpha\right)^{\frac{1}{2}}
$$

Similarly

$$
h=\frac{1}{\sin \theta}\left(\frac{\bar{E}(\theta)^{2}}{\bar{E}(0)^{2}}-\cos ^{2} \theta\right)^{\frac{1}{2}}
$$

The values for $\alpha, \dot{\theta}$ selected in this study were $0,90^{\circ}$, as suggested by Jorgensen $n^{|33|}$. Th.' probes were visually aligned with the flow and slight misalignments had little effect on the results since large angles were selected (i. e. $0,90^{5}$ ). The values of the sensitivity coefficients obtained in this study were $k=0.236$ and $h=1.174$ for an average of five different probes (normal and slanted).

\subsection{Experimentation on the B.L.C. Apparatus}

\subsubsection{Mass Flow Rate Measurements}

The suction and blowing flow rates were calculated by using the measured pressure differences across the orifice-plates in the following equation ${ }^{\mid 10}$;

$$
\dot{M}=359.1 \times c \times z \times d^{2} \times E \times \overline{\sqrt{h}} \times \sqrt{w}
$$

where

$$
\begin{aligned}
& \dot{M}=\text { mass flow rate, }(\mathrm{lbm} / h r) \\
& c=\text { discharge coefficient }=0.608 \text { here } \\
& d=\text { orifice diameter, (inches) } \\
& E=\text { velocity of approach factor }=\frac{1}{\sqrt{1-m^{2}}} ; m=\left(\frac{d}{P}\right)^{2} \\
& D=\text { pipe diameter, (inches) }
\end{aligned}
$$



$d=$ cylinder diamet,er

The $C_{\mu}$ values attained in this study were $0.1,0.2$ and 0.22 for the cylinder jet and $(1.11$ for the plane wall-jet. A calibration between the orifice-plate pressure difference and the plane wall plenum chamber pressures wes established at actual operating temperature and freestream velocity for each cylinder momentum coefficient $\left(C_{\mu_{c}}\right)$ and wall-jet momentum coefficient $\left(C_{\mu_{w}}\right)$ combination. During actual test rünning, the orifice-plates were removed from the blowing air supply lines and the $C_{\mu}$ 's were determined using the measured plenuis chamber pressures. This procedure was adopted to reduce blower power requirements ihu' minimizing heating of the blowing air and attendant errors in the hot-wire datis. Figur" 17 shows the calibration curves for both cylinder and wall slots. A computer program. MFLOW, was developed to calculate, as accurately as possible, both 1. mass flow ral.. and the slot thickness of the cylinder and wall slots. The jet exit velocities, $V$, calculated from equation 3.13 were approximately checked with pltot tube ( $1 \mathrm{~mm}$ O.D.) measurements placed at the slot exits. the slot thicknesses were also approximately checked by feeler gauges. All the necessary measured input parameters, a short description and a listing of the "MF.LOW" program are shown in Appendix C.

\subsubsection{End-Wall Boundary Layer Control}

When the blowing on the cylinder is activated, the separation of the flow is not symmetrical about the cylinder-forebody chord. Thus the flow conditions are similar to those observed in circulation controlled wings (see Figure 1), where the separation of the flow at the side of the slot occurs later than the separation from the other side. When a circulation controlled two-dimensionat wing section is tested in a wind tunnel, the circulation around it causes a tendency for the vorticity to be shed where the wing penetrates the boundary layer on the floor and ceiling of the wind tunnel. Thus the bound vorticity shed downstream of the wing leading edge introduces three-dimensional effects into the flow.

In this study, in order to ensure two-dimensional flow conditions downstream at the forebody and at the vicinity of the cylinder slot exit, suction was applied to the porous end-walls. The suction intended to reduce the thickness of the boundary layer and redure tendency-for bound vorticity, near the cylinder (i.e. where vorticity is more pronounced). The amount of suction required to ensure two-dimensionality of the flow, with only the circulation controlled cylinder in símilar flow conditions as in the present study, was de termined by Gooden ${ }^{[27]}$. He describes that the suction requirement corresponds to approximately two times the approaching turbulent boundary layer deficit mass flow and in his 
case, $V_{n} / U_{x_{0}}$ was equal to about 0.015 fot each plate, where $V_{n}$ is the velocity component normal to the porous end plate. In this study, although the suction requirements were lighter due to the presence of the forebody, limitations of suction equipment did not allow for higher suction rates to be achieved. The $V_{n} / U_{\infty}$ 'zatio here was about 0.013 for each porous plate.

\subsubsection{Flow Quality Checks}

This section includes descriptions of a number of flow quality checks performed in this study, in order to provide strong direct or indirect evidence that the flow downstream of the cylinder. slot, achieved reasonably two-dimensional conditions. Good flow twodimensionality is essential if the data are to be used for validation of two-dimensional flcw calculation methods.

\section{Flow Alignment and Visualization}

With the test section in the wind tunnel circuit carefully leveled in all directions and clamped in place, separate leveling of all B.L.C. apparatus components were necessary. First the two end-walls porous plates were faired into the section's end-walls and the cylinder was adjusted normal to the porous end plates. The plane wall-jet in this arrangement was at a fixed distance of 5.25 in from the center of the cylinder. The test section walls were all smooth. The forebody was fitted upstream of the cylinder and fixed in place with its leading edge plane normal to the section's end-walls as shown in Figures 8, 11 and 12 .

Having ensured that the apparatus was aligned with the tunnel freestream flow direction, a grid of tufts of 0.75 in long black thread were taped at various positions along the surface of the forebody. This was done primarily to ensure that the boundary layer developing on the forebody did not separate before it reached the cylinder jet exit, and also to examine the spanwise flow development on the forebody. No separation of the flow was detected and the tufts failed to indicate any spanwise flow irregularities.

Another tuft visualization scheme was used downstream of the cylinder and wall-jets. Tufts of 0.75 in long black thread were glued in a square grid formation 0.75 in apart as shown in Figures 31 to 33 . One grid covered the yortical side wall of the working section for a distance of 11 in downstream of the wall-jet. Grids of tufts on both the ceiling and floôr of the working section covered an area of 11.5 in $\times 6$ in just downstream of the porous end plates, as shown in Figures 31 to 33. 
With the tufts in place, a series of trial and error experiments were conducted is determine the best roughness element configuration for tripping, and this making fuller. the boundary layer that developed on the forebody. Fripping wires of $1.2 \mathrm{~mm}$ and $2.1 \mathrm{~mm}$ O.D's and strips of dymo-tape with a capital $\mathrm{V}$, were first uied at several upstream positimn:s across the entire $50 \mathrm{~cm}$ span of the forebody. By observing the movementerf the tufts, which the wind tunnel was switched on and at the highest cylinder jet momentum corfficintit int $C_{\mu_{c}}=0.22$, it was possible to evaluate the effectiveness of the tripping devices. It wals fius: : that tripping of the boundary tayer upstream improved the two-dimensionalicy of thin flin mainly because the regulting turbulent boundary layer is more stable. A considerable $11 \ldots$. was'devoted in obtaining the best roughness configuration. The roughness arrangentern! axtopted for further experimentation consisted of a $50 \mathrm{~cm} \times 23 \mathrm{~cm}$ piece of sand parers mounted on the leading edge of the forebody. It should be noted that because the primary concern in this study was to obtain good flow twu-dimensionality conditions downstream of the cylinder jet.

Surface flow visualization on the cylinder was also attempted in order to provide evidence on the spanwise uniformity of the separation point. A thin film of glycerin with titanium oxide mixture, 3 applied just downstream of the cylinder slot exit. The static pressure taps on the cylif der were previously covered with a strip of adhesive tape starting at a distance $\theta=12^{\circ}$ from the jet exit. The flow was kept until the pattern developed by the glycerin mixture remained unchanged. Then, the flow was turned off so that the pattern could be photographed. A discussion on the flow visualization results will follow in Chapter 5. 。

\section{Pressure Measurements}

Static pressure measurements was another means that was used for providing evidence about the flow two-dimensionality. Spanwise static pressure at distances of $x=7$, in and $x=11$ in on the side wall downstream of the wall-jet exit, were recorded at all $C_{\mu_{r}}, C_{\mu_{\infty}}$ combinations with an accuracy of about $2 \%$, using water filled manometers. Results and discussionjof these measurements will follow in Chapter 5.

\section{Still Air Measurements}

Previous work" of Kind et al. ${ }^{[38]}$ on E.L.C. apparatus, had revealed that random short. wavelength fluctuations of the maximum total pressure across the span of the cylinder fet occurred under still air conditions. 
In this study, this"effect was also examined with the eyinder jet issuing in still air silrrounding a pitot tube of $1 \mathrm{~mm}$ I.D. which was traversed across the central $40 \mathrm{~cm}$ of the cylinder span and at three downstreem stations; namely at $\theta=32^{\circ}, 62^{\circ}$, and $92^{\circ}$ from the cylinder 'slot exit. Simultaneous measurements of the maximum total pressure and the cylinder plenum pressure were taken at $0.5 \mathrm{~cm} \pm 3 \%$, spanwise intervals using two single ' tube tilting manometers.

The pressures could be estimated with an accuracy of about $1.5 \%$. Results and discussion on these measurements will follow in Chapter 5 .

In still air surroundings, the static pressure distribution on the cylinder was also recorded using the multitube manometer board, with an accuracy of about 2\%. These - results will be later compared to those of Fekete ${ }^{[23]}$.

\section{Spanwise Mean Velocity Profile Measurements}

Spanwise mean velocity measurements were another means used for providing evidence on the flow two-dimensionality downstream of the cylinder jet exit and in the close vicinity of the cylinder. A normal hot-wire probe was traversed through the shear layer that developed on the cylinder, at various downstream stations and at spanwise distances of $z=-1,-14$ and $+14 \mathrm{~cm}$. The cylinder momentum coefficients were $C_{\mu_{c}}=0.1,0.2$ and 0.22 while the wall-jet momentum coefficientiwas constant at $C_{\mu_{*}}=0.11$. Results and discussion will follow in Chapter 5 .

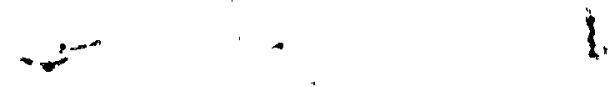

\subsubsection{Repeatability and Operating Flow Conditions}

\section{Control of Flow Parameters}

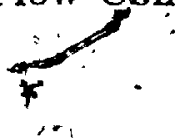

The wind tunnel freestream velocity $\left(U_{\infty}\right)$ was monitored via a tilting micro-manometer as described in Section 2.2.8. In this study, the freestream,velocity was held constant at $U_{\infty}=21 \mathrm{~ms}^{-1}$, a value set by Kind et al. ${ }^{[38]}$ in all experiment runs. The primary reason for choosing this particular freestream velocity was that cylinder momentum coefficients of $C_{\mu_{0}}=0.22$ maximum could be attained with the ayailable equipment. The Reynolds number with respect to the cylinder diameter, corresponding to this velocity was about 180,000. The variation of $U_{\infty}$ was less than $1 \%$ durin' a typical experimental run of one hour. The side wall jet momentum coefficient was also held constant at $C_{\mu_{\infty}}=0.11$ in all experiment runs. This was because earlier experiments by Gooden ${ }^{[27]}$ did not show significant effect of the wall-jet momentum coefficient on the cylinder velocity distribution 
The effect of higher $C_{\mu_{w}}$ on the mean velocity and longitudinal turbulence at $\partial=1$ "' on the cylinder has been also investigated in this study. The cylinder jet momentum coefficient. $C_{\mu_{c}}$, values at ${ }^{\prime}$ ined in the present experiments were $0.1,0.2$ and 0.22 . A calibration relationship between the mass-flow rate supplied to the wall and cylinder plenum chambers and the corresponding pressure in the plenum (described in Section 3.2.1), allowed cluse monitoring of the $C_{\mu_{e}}$ and $C_{\mu_{*}}$ via manometers.

The suction rate through each porous end-wall plate was held constant at approx mately $60 \mathrm{c.f} . \mathrm{m}$. With all the flow parameters set accordingly sufficient time, approximately * five minutes, was allowed for steady-state flow conditions to be reached. By simultinteously monitoring the flow parameters and the static pressure distribution downstream of the cylinder slot, it was found the same flow conditions could be repeated accurately. Me:isurements of mean velocity profiles at the same station and at different time periods verified the repeatability of the flow conditions.

\section{Temperature Measurements}

One of the major difficulties in using hot-wires for measurements is the effect of fhuid temperature changes on the heat transfer from the sensors. Since the hot-wire responds both to velocity and temperature fluctuations, elimination of the latter helps to ensure accurate velocity measurements.

Although the flow temperature in the present study could not be fully controlled, due to limitations of the equipment, an attempt was made to estimate the temperature. changes. Early experiments had shown that the wind tunnel freestream temperature varied a maximum of $2^{\circ} C$ over a period of two hours. The air temperature inside the side wall $-j a t$ plenum chambr was monitored with an accuracy of $\pm 1^{\circ} \mathrm{C}$ via a thermocouple. In a typical experimental run, at $C_{\mu_{\infty}}=0.11$, the side wall-jet plenum temperature was about $7^{\circ} C$ higher than the freestream air temperature The temperature in the cylinder plenum at $C_{\mu_{c}}=0.1$ was $5^{\circ} \mathrm{C}$ higher than the freestream air temperature, at $C_{\mu_{r}}=0.2$ it was about $10^{\circ} \mathrm{C}$ higher and at $C_{\mu_{c}}=0.22$ it was about $15^{\circ} \mathrm{C}$ higher than freestream air temperature. Since the aforementioned increases in temperatures was the result of heating by the blower motors, which could not be controlled, in all experimental runs it was tried to obtain the same temperature differences. This would ensure good repeatability in the measurements even if errors were to be introduced by the temperature changes.

- Bradshaw ${ }^{[6]}$ describes that one means of minimizing temperature effects is to run the 
wire at as high a temperature as possible. Hence, the hot-wire sensor temperature was set (1) $: 00^{\circ} \mathrm{C}$, which corresponds to an overheat ratio of 1.9 in all experiment runs. Higher overheat ratios could not pe sustained by the tyungsten wires used in this study. Although the plenum chamber air temperatures and the freestream air temperature could easily be monitored during the experiment, the actual temperature of the flow resulting from the mixing of the cylinder jet air with the freestream air was not known. According to the TSI anemometer manual, the overheat ratio of a hot-wire sensor is given by the following relation:

$$
\text { Overheàt ratio }=\frac{R_{h}}{R_{c}}=1+q_{c}\left(T_{s}-T_{e}\right)
$$

where

$q_{c}=$ temperature coefficient for refistance of sensor

$\left(\approx 0.0058 /^{\circ} \mathrm{C}\right.$ for the tungsten wire used in this experiment)

$h_{h}=$ operating resistance of sensor ${ }^{*}$ :

$R_{c}=$ rèsistance of sensor when at fiuid temperature

$T_{s}=$ operating temperature of sensor

$\therefore T_{\epsilon}=$ temperature of the operating environment

An estimation of the temperature difference between the cylinder wall-jet air and the freestream air may be obtained by" rewriting equation 3.15 , as;

$$
\Delta T=\frac{\Delta R}{R_{o} q_{c}}
$$

where

* $\Delta T=$ temperature difference between the freestream and cylinder wall-jet air

$\Delta R=$ difference in cold wire resistance at $\Delta T$

$R_{\circ}=$ Cold wire resistance at reference temperature :

Thereference temperature and the corresponding, cold wire resistance were assutmed the same as those of the freestream air. Thus, an estimation of the actual flow temperature was obtained by traversing a cold-wire through the boundary layer downstream of the cylinder jet. The cold resistances were measured by adjusting the variable "Probe Resistance Decades" on the anemometer until the meter needle did not deflect when the ". 
"Res Meas" control was quickly pressed and released. Results and discusion on tem. perature effects will follow in Chapter 5.

\section{Zeroing of the Hot-Wire Probes}

The traversing linkage mechanism used for these experiments has been described in Section 2.2.6 and it is shown in Figures 10 and 11. The hot-wire probes were brought in the first measuring position, "zeroed", with good repeatability by following the "mpir. 11 experimental procedure: with the anemometer in the "Stand-By" position oft nu ftris the prongs of a normal hot-wire probe were moved tangentially to the surface When th.. prongs touched the stee) cylinder surface, the movement was stopped and "the orientistin. of the sensor was checked visually u'sing a mirror. The protractor system in the back of the probe holder tube was adjusted to $90^{\circ}$ roll angle $\left(\psi=90^{\circ}\right)$ at this position.

The movement was then reversed and the prongs were moved an average of $0.05 \mathrm{~mm}$ from the cylinder surface. This position was definedas the first measuring point. When 40 slanted probes were used with their prong plane normal to the cylinder surface as shown in Figure 18, the first measuring point is defined at $0.55 \mathrm{~mm}$ from the cylinder surface to the probe axis. . Before proceeding with the actual measurements, the bridge voltage at zero flow $\bar{E}_{0}$ was checked with the $\bar{E}_{0}$ obtained during calibration. All distances needed for $\ddots$ solving the kinematics of the traversing linkage were measured with a micrometer.

\section{Mean Velocity and Turbulence Measurements}

Mean velocity and turbulence data were collected at eight stations downstream of ine cylinder slot exit; namely at $\theta=2^{\circ}, 12^{\circ}, 22^{\circ}, 42^{\circ}, 52^{\circ}, 62^{\circ}$ and $72^{\circ}$. The spanwise - position of the probe was $Z=-1 \mathrm{~cm}$ from.the centerline, in all experiments. The choics of $Z=-1 \mathrm{~cm}$ as the measuring position at all stations was because three-dimensionality effects would be less severe at the midspan of the cylinder. Moreover, it was necessary to keep some distance from the static pressiure ţaps.located on the surface of the cylinder to eliminate possible flow disturtances resulting from them.

A large number of data points were collected at each measuring station in an attempt. to better define the minima and maxima of botho the mean velocity and turbulence profiles. The need for a large number of data points at measuring stations close to the slot exit was more pronounced because there the gradients of t'do profiles were larger and the shear layer was thinner. The computel programs developed to collect data and to traverse the . probes allowed changes of the traversing step size during the experiments. "Thus, once 
the probes were "zeroed" and the flow conditions established, the experiment was fully computer controlled and it coun be easily interrupted.

Measurements usin Normal Probes :

Mean velocities were measured using a single normal hot-wire probe. The prong plane of the probe was parallel to the cylinder surface, $\dot{\psi}=90^{\circ}$, as shown in Figure 18 . With this arangement, a better spatial resolution could be achieved than with $45^{\circ}$ slated proe and more data could be collected in the vicinity of the cylinder surface.

The ADACS computer was programmed to sample at the optimum, rate described in Section 3.1.2. The ADACS computer program version, "U\&RMS", calculated the àctual $Y$ distance of the probe from the cylinder surface, the mean velocity, $u$, and the longitudinal turbulence, $\sqrt{u^{\prime 2}}$. A short description and a listing of the program "U\&RMS" is shown in Appendix D.

\section{Measurements using $45^{\circ}$ Slanted Probes}

Th shear stresses $\overline{u^{\prime} v^{\prime}}$ were measured with slanted probes. The probe orientation was such that the probè prongs plane was normal to the cylinder surface as shown in Figure 18. Once the probe was traversed at this orientation $\left(\psi=0^{\circ}\right)$, it was brought back to the zero position, rolled $180^{\circ}$ and the same procedure repeated.

- The ADACS cornputer program version “M\&RMS", was also designed to sample at

$\therefore \quad$ the optimum rate and calculated the distance, $Y$, from the cylinder surface and displayed the $\bar{E}_{b r}$ and $\sqrt{\bar{e}_{b r}}$ at eâch measuring point. Part of the program "M\&RMS" is described in Appeñdix D.

* During the experimental runs, thWADACS system and all the supporting instruments were closely checked to ensure that the experiment proceeded satisfactorily. By observing the hot-wire signal on the oscilloscope, it was possible to detect experimental malfunctions. .

In such a case, the traversing was repeated. All the data were transfered to the University's mainframe compuiter system for processing. 


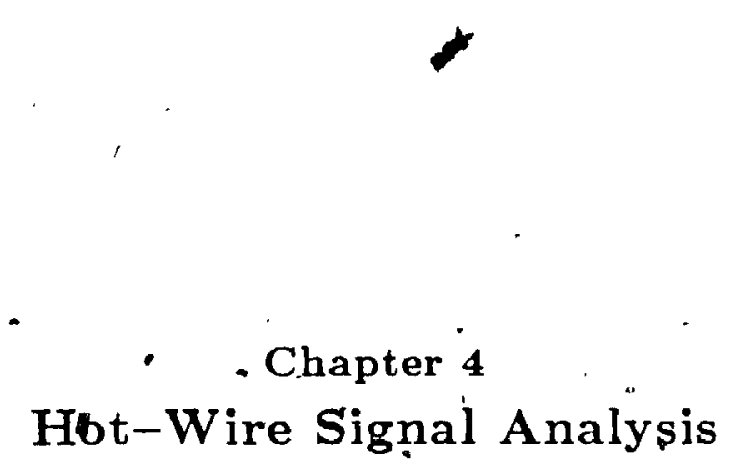

\subsection{Introduction}

Hot-wire anemometer signal anelysis has been the subject of many reseirch proy $\ldots 1.1: 1$ the past. One of the most notable is the analysis by Acrivlellis 1 ! . He describes that with hiv method, a conventional hot-wire anemometer and a commercially available slanting init wire probe as well as a simple measuring setup may be used to determine all velotit! ani Reynolds stress components, in multi-dimensional flow fields of any turbulent intensity with no sacrifice of accuracy. Acrivlellis's method has been extensively studied by Youraknini who found errors in its formulation. Therefore, he ${ }^{[55]}$ directed his efforts towards the derivation of a similar hot-wire signal analysis with the orientation of the hot-wire probe axis normal to the freestream fiow direction.

One of the tasks of this thesis was to derive hot-wire equations for the coordinate" system shown in Figure 19, following similar guidelines as those icribed by $Y_{C}$ wakim In the present work the probe axis is approximately aligned with the nominal fow arection. This would enable measurements of all mean velocity and Reynolds stress components in the flows presented in this study, by using a single $45^{\circ}$ slant probe or a combination of $45^{\circ}$ slant and normal hot-wire probes.

\subsection{Derivation of Hot-Wire Equations fur 3-D Flows}

The method described here is based on masurements with a single $45^{\circ}$ hot-wire prols" rotated at as many angles as are required for determining all the unknowns, or a combination of slant and normal probes. The resulting system of equations relating the square out put hoț-wire signal to mean or turbulence quantities can be solved simultaneously to determme. these quantities. Figure 19 shows a $45^{\circ}$ slant hot-wire probe in an arbitrary angular posifion in the flow field. The velocity components of this field are $u+u^{\prime}, v+v^{\prime}$ and $w+w^{\prime}$ parallpl to the axes $x, y$ and $\varepsilon$ of the inertial coordinate system. Primes indicate fuctuating components. The $\xi, " s, \eta$ coordinate system of Figure 19 is a probe-based system $\Gamma$ he Faxis or the probe-based velocity, $U_{N}$, is perpendicular to the hot-wire, forming angle v. "with the probe axis. "The angle,' $\alpha$, is $45^{\circ}$ for a $45^{\circ}$ slant probe or $0^{\circ}$ for a normal protse 
The axes $S$ and $\eta$, and the corresponding velocity components, $u_{T}$ and $u_{\text {p }}$, are defined parallel and bi-normal to the hot-wire. Each angular position of the hot-wire is obtained through rotation of the $\alpha^{\circ}$ slant probe about its axis. The probe-based coordinate system moves with it. The transformation from inertial to probe-based coordinate system requires a rotation of the $x, y, z$ coordinate system on the $x$-axis, probe-axis, through the angle $\psi$, and on the $z$-axis through the angle $\alpha$. Since this is an orthogonal transformation, the velocity components are transformed in the same way as the coordinates. Hence, the instantaneous velocity components are;

$$
\begin{aligned}
& u_{N}=\left(u+u^{\prime}\right) \cos \alpha+\left[\left(v+v^{\prime}\right) \cos \psi-\left(w+w^{\prime}\right) \sin \psi\right] \sin \alpha \\
& u_{T}=-\left(u+u^{\prime}\right) \sin \alpha+\left[\left(v+v^{\prime}\right) \cos \psi-\left(w+w^{\prime}\right) \sin \psi\right] \cos \alpha \\
& u_{B}=\left(v+v^{\prime}\right) \sin \psi+\left(w+w^{\prime}\right) \cos \psi
\end{aligned}
$$

where $u_{N}, u_{T}, u_{B}$ are the normal, tangential and binormal velocity components defined previously.

The effective cooling velocity acting on the sensor depends on the yaw and pitch angles that the sensor makes with the freestream flow direction. Jørgensen ${ }^{[24]}$ has defined the : effective cooling velocity acting on the sensor as;

$$
\text { - . } u_{e f f}^{2}=u_{N}^{2}+k^{2} u_{T}^{2}+h^{2} u_{B}^{2}
$$

where $k$ is the yaw factor and $h$ is the pitch factor.. The method determination of these factors has been mentioned in Section 3.1.4. .

The linear relationship between effective cooling velocity and hot-wire output voltage $\because$ is;

2.

$$
E=S u_{e f f}
$$

where $S$ is the slope of the linearized plot of $E$ vs. ueff. 
From equations 4.4 and 4.5 ,

$$
u_{e f f}=\left(u_{N}^{2}+k^{2} u_{T}^{2}+h^{2} u_{B}^{2}\right)^{\frac{1}{2}}
$$

or

$$
\begin{aligned}
u_{e f f}= & {\left[\left\{\left(u+u^{\prime}\right) \cos \alpha+\left[\left(v+v^{\prime}\right) \cos \psi-\left(w+w^{\prime}\right) \sin \psi^{\prime}\right] \sin \alpha\right\}^{2}\right.} \\
& +k^{2}\left\{-\left(u+u^{\prime}\right) \sin \alpha+\left[\left(v+v^{\prime}\right) \cos \psi-\left(w+w^{\prime}\right) \sin \psi^{\prime}\right] \cos \alpha\right\}^{2} \\
& +h^{2}\left\{\left(v+v^{\prime}\right) \sin \psi+\left(w+w^{\prime}\right) \cos \psi^{2}\right]^{\frac{1}{2}} \\
= & \frac{E(\psi, \alpha)}{S}
\end{aligned}
$$

The square root term of equation 4.7 is not expanded into a binomial series and then time-averaged as in the case of the conventional method of determination, but it is first squared and thereafter time-averaged. Then, for the squared output signal;

$$
\frac{\overline{E^{2}}(\psi, \alpha)}{S^{2}}=a u^{2}+b v^{2}+c w^{2}+d u v+f v w+g u w
$$

or

$$
\frac{\bar{E}(\psi, \alpha)}{S}=\sqrt{a u^{2}+b v^{2}+c w^{2}+d u v+f v w+g u w}
$$

where

$$
\begin{aligned}
a & =\cos ^{2} \alpha^{2}+k^{2} \sin ^{2} \alpha \\
\quad & =\sin ^{2} \alpha \cos ^{2} \psi+k^{2} \cos ^{2} \alpha \cos ^{2} \psi+h^{2} \sin ^{2} \psi \\
c & =\sin ^{2} \alpha \sin ^{2} \psi+k^{2} \sin ^{2} \psi \cos ^{2} \alpha+h^{2} \cos ^{2} \psi \\
d & =\sin 2 \alpha \cos \psi-k^{2} \sin 2 \alpha \cos \psi \\
f & =-\sin 2 \psi \sin ^{2} \alpha-k^{2} \sin 2 \psi \cos ^{2} \alpha+h^{2} \sin 2 \psi \\
g & =k^{2} \sin \psi \sin 2 \alpha-\sin \psi \sin 2 \alpha
\end{aligned}
$$

The only assumption involved in obtaining equation 4.8 was that for small turbulence intensity $(\approx 20 \%)$, the turbulent terms were considered very small" compared with the time-mean velocity terms and they were omitted. Detailed intermediate steps involved in obtaining equation 4.8 from the expansion of equation 4.7 are shown in Appendix $F$.

From equation 4.8 one can get the time-mean velocity components of flow from mean $(\bar{E})$ output signal of the hot-wire anemometer. To determine the three mean velocity 
components from equation 4.8 , one has to establish a system of three independent linear cquations, by taking meașurements for three suitable $\psi$ values with a slanted hot-wire probe at each point in the flow.

If ' $e$ ' is corlsidered to be the fluctuating component of the linearized output hot-wire a signal, it can be related to the linearized instaftaneous and time averaged signals $E$ and $\bar{E}$ as; :

$$
\frac{e(\psi, \alpha)}{S}=\frac{E(\psi, \alpha)-\bar{E}(\psi, \alpha)}{S}
$$

Substituting expressions of $E(\psi, \alpha) / S$ and $\bar{E}(\psi ; \alpha) / S$ into equation 4.9 and after manipulation and time averaging we can arrive at the final form to be used in the data reduction.

$$
\begin{aligned}
& \text { Therefore, } \\
& \text { where } \frac{\overline{e^{2}}(\psi, \alpha)}{S^{2}}=\frac{1}{4 Q^{2}}\left[a_{1}^{2} \overline{u^{\prime 2}}+b_{1}^{2} \overline{v^{\prime 2}}+c_{1}^{2} \overline{w^{\prime 2}}+2 a_{1} b_{1} \overline{u^{\prime} v^{\prime}}+2 \dot{a_{1}} c_{1} \overline{u^{\prime} w^{\prime}}+2 b_{1} c_{1} \overline{v^{\prime} w^{\prime}}\right] \\
& Q=\frac{\bar{E}(\psi, \alpha)}{S}=\left[a u^{2}+b v^{2}+c w^{2}+d u v+f v w+g u w\right]^{\frac{1}{2}} \\
& a_{1}=2 a u+d v+g w \\
& b_{1}=2 b v+f w+d u \\
& c_{1}=2 c w+g u+f v
\end{aligned}
$$

The assumption involved in this analysis was for low turbulence intensity $(\approx 20 \%)$. Higher order terms such as $\overline{u^{\prime 4}}, \overline{u^{\prime 2} v^{\prime 2}}, \overline{u^{\prime} v^{\prime 2}}$ etc. were neglected since for low turbulence intensity, they will be small compared with the terms obtained in equation $4.10^{\prime}$

Equation 4.10 gives results for the Reynolds stresses (in 3-D flow). This -expression is valid for both slanted and normal hot-wire probes, provided that the turbulence intensity is less than 20\%. Coefficients $a_{1}, b_{1}, c_{1}$ should be computed at each local position in. the flow field and for every probe orientation angle, $\psi$. Finding all six Reynolds stress components requires that a system of six linear independent equations be established by selecting suitable $\alpha$ and $\psi$ values for the hot-wire probe. Detailed intermediate steps involved in arriving at equation 4.10 from equation 4.9 are shown in Appendix $F$. 
$\checkmark$

、4.2.1 Determination of Mear and Fluctuating Quantities at Differnt $(2,11)$

Position $1\left(\psi=0^{\circ}, \alpha=45^{\circ}\right)$

Erom equation 4.8;

$$
\begin{aligned}
a & =\frac{1}{2}+\frac{1}{2} k^{2}=\frac{1}{2}\left(1+k^{2}\right) \\
b & =\frac{1}{2}+\frac{1}{2} k^{2}=\frac{1}{2}\left(1+k^{2}\right) \\
c & =h^{2} \\
d & =1-k^{2} \\
f & =0 \\
g & =0
\end{aligned}
$$

Therefore,

or for 2-D flow;

$$
\text { - } \left.\frac{E(0,45)}{, S}=\left[\frac{1}{2}\left(1+k^{2}\right) u^{2}+\frac{1}{2}\left(1+k^{2}\right) v^{2}+h^{2} w^{2}+7-k^{2}\right) u v\right]^{\frac{1}{2}} .
$$

$$
\frac{E(0,45)}{S}=\left[\frac{1}{2}\left(1+k^{2}\right) u^{2}+\frac{1}{2}\left(1+k^{2}\right) v^{2}+\left(1-k^{2}\right) u v\right]^{\frac{1}{2}}
$$

From equation 4.10 ;

$$
\begin{aligned}
& a_{1}=2 a u+d v+g w \\
& b_{1}=2 b v+f w+d u \\
& c_{1}=2 c w+g u-f v
\end{aligned}
$$

or

$$
\begin{aligned}
& a_{1}=\left(1+k^{2}\right) u+\left(1-k^{2}\right) v \\
& b_{1}=\left(1+k^{2}\right) v+\left(1-k^{2}\right) u \\
& c_{1}=2 h^{2} w
\end{aligned}
$$

$$
\frac{\overline{e^{2}}(0,45)}{S^{2}}=\frac{1}{4 Q^{2}}\left[a_{1}^{2} \overline{u^{\prime 2}}+b_{1}^{2} \overline{v^{\prime 2}}+c_{1}^{2} \overline{w^{\prime 2}}+2 \overrightarrow{a_{1}} b_{1} \overline{u^{\prime} v^{\prime}}+2 a_{1} c_{1} \overline{u^{\prime} w^{\prime}}+2 b_{1} c_{1} \overline{v^{\prime} w^{\prime}}\right]
$$

or for 2-D flow;

$$
\frac{\overline{e^{2}}(0,45)}{S^{2}}=\frac{1}{4 Q^{2}}\left[a_{1}^{2} \overline{u^{\prime 2}}+b_{1}^{2} \overline{v^{\prime 2}}+2 a_{1} b_{1} \overline{u^{\prime} v^{\prime}}\right]
$$


Position $\left.2(\psi=90)^{n}, \quad x=15^{\circ}\right)$

- '

From equation 4.8 ;

$$
\begin{aligned}
a & =\frac{1}{2}\left(1+k^{2}\right) \\
b & =h^{2} \\
c & =\frac{1}{2}\left(1+k^{2}\right) \\
d & =0 \\
f & =0 \\
g & =k^{2}-1 . \\
\frac{E(90,45)}{S}=\left[\frac{1}{2}\left(1+k^{2}\right) u^{2}\right. & \left.+h^{2} v^{2}+\frac{1}{2}\left(1+k^{2}\right) w^{2}+\left(k^{2}-1\right) u w\right]^{\frac{1}{2}}
\end{aligned} .
$$

or for 2-D flow:

$$
\frac{\bar{E}(90 ; 45)}{S}=\left[\frac{1}{2}\left(1+k^{2}\right) u^{2}+h^{2} v^{2}\right]^{\frac{1}{2}}
$$

From equation 4.10 ;

$$
\begin{aligned}
& a_{1}=\left(1+k^{2}\right) u+\left(k^{2}-1\right) w \\
& b_{1}=2 h^{2} v \\
& c_{1}=\left(1+k^{2}\right) w+\left(k^{2}-1\right) u \\
& \frac{e^{2}}{\frac{190,45)}{S^{2}}}=\frac{1}{4 Q^{2}}\left[a_{1}^{2} \overline{u^{\prime 2}}+b_{1}^{2} \overline{v^{\prime 2}}+c_{1}^{2} \overline{w^{\prime 2}}+2 a_{1} b_{1} \overline{u^{\prime} v^{\prime}}+2 a_{1} c_{1} \overline{u^{\prime} w^{\prime}}+2 b_{1} c_{1} \overline{v^{\prime} w^{\prime}}\right]
\end{aligned}
$$

or for 2-D flow;

$$
\frac{\overline{e^{2}}(90,45)}{S^{2}}=\frac{1}{4 Q^{2}}\left[a_{1}^{2} \overline{u^{\prime 2}}+b_{1}^{2} \overline{v^{\prime 2}}+2 a_{1} b_{1} \overline{u^{\prime} v^{\prime}}\right]
$$




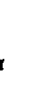

Position $3\left(\psi=180^{\circ}, \alpha=45^{\circ}\right)$

From equation 4.8;

$$
\begin{aligned}
a & =\frac{1}{2}\left(1+k^{2}\right) \\
b & =\frac{1}{2}\left(1+k^{2}\right) \\
c & =h^{2} \\
d & =k^{2}-1 \\
f & =0 \\
g & =0 \\
-\quad & \frac{E(180,45)}{S}=\left[\frac{1}{2}\left(1+k^{2}\right) u^{2}\right.
\end{aligned}
$$

or for 2-D flow;

$\frac{\bar{E}(180,45)}{S}=\left[\frac{1}{2}\left(1+k^{2}\right) u^{2}+\frac{1}{2}\left(1+k^{2}\right) v^{2}+\left(k^{2}-1\right) u v\right]^{\frac{1}{2}}$

From equation 4.10;

$$
\begin{aligned}
& a_{1}=\left(1+k^{2}\right) u+\left(k^{2}-1\right) v \\
& b_{1}=\left(1+k^{2}\right) v+\left(k^{2}-1\right) u \\
& c_{1}=2 h^{2} w
\end{aligned}
$$

$$
\frac{\overline{e^{2}}(180,45)}{S^{2}}=\frac{\mathrm{i}}{4 Q^{2}}\left[a_{1}^{2} \overline{u^{\prime 2}}+b_{1}^{2} \overline{v^{\prime 2}}+c_{1}^{2} \overline{w^{\prime 2}}+2 a_{1} b_{1} \overline{u^{\prime} v^{\prime}}+2 a_{1} c_{1} \overline{u^{\prime} w^{\prime}}+2 b_{1} c_{1} \overline{v^{\prime} w^{\prime}}\right]
$$

or for 2-D flow;

$$
\frac{\overline{e^{2}}(180,45)}{S^{2}}=\frac{1}{4 Q^{2}}\left[a_{1}^{2} \overline{u^{\prime 2}}+b_{1}^{2} \overline{v^{\prime 2}}+2 a_{1} b_{1} \overline{u^{\prime} v^{\prime}}\right]
$$


35

Position 4 $\left(\psi=270^{\circ} . \alpha=45^{\circ}\right)$

From equation 4.8;

$$
\begin{aligned}
a & =\frac{1}{2}\left(1-k^{2}\right) \\
b & =h^{2} \\
c & =\frac{1}{2}\left(1-k^{2}\right) \\
d & =0 \\
f & =0 \\
g & =1-k^{2}
\end{aligned}
$$

$$
\frac{\bar{E}(270,45)}{S}=\left[\frac{1}{2}\left(1+k^{2}\right) u^{2}+h^{2} v^{2}+\frac{1}{2}\left(1+k^{2}\right) w^{2}+\left(1-k^{2}\right) u w\right]^{\frac{1}{2}}
$$

or for 2-D flow;

$$
\frac{\bar{E}(270,45)}{S}=\left[\frac{1}{2}\left(1+k^{2}\right) u^{2}+h^{2} v^{2}\right]^{\frac{1}{2}}
$$

From equation 4.10;

$$
\begin{aligned}
& a_{1} \doteq\left(1+k^{2}\right) u+\left(i-k^{2}\right) w \\
& b_{1}=2 h^{2} v \\
& c_{1}=\left(1+k^{2}\right) w+\left(1-k^{2}\right) u \\
& \frac{\overline{e^{2}}(270,45)}{S^{2}}=\frac{1}{4 Q^{2}}\left[a_{1}^{2} \overline{u^{\prime 2}}+b_{1}^{2} \overline{v^{\prime 2}}+c_{1}^{2} \overline{w^{\prime 2}}+2 a_{1} b_{1} \overline{u^{\prime} v^{\prime}}+2 a_{1} c_{1} \overline{u^{\prime} w^{\prime}}+2 b_{1} c_{1} \overline{v^{\prime} w^{\prime}}\right] \\
& \text { or for 2-D flow; : } \\
& \frac{\overline{e^{2}}(270,45)}{S^{2}}=\frac{1}{4 Q^{2}}\left[a_{1}^{2} \overline{u^{\prime 2}}+b_{1}^{2} \overline{v^{\prime 2}} * 2 a_{1} b_{1} \overline{u^{\prime} v^{\prime}}\right]
\end{aligned}
$$


Position $5\left(\psi^{-}=45^{\circ}, \alpha=45^{\circ}\right)$

From equation 4.8 ;

$$
\begin{aligned}
& a=\frac{1}{2}\left(1+k^{2}\right) \\
& b=\frac{1}{4}\left(1+k^{2}\right)+\frac{1}{2} h^{2} \\
& c=\frac{1}{4}\left(1+k^{2}\right)+\frac{1}{2} h^{2} \\
& d=0.707\left(1-k^{2}\right) \\
& f=-\frac{1}{2}\left(1+k^{2}\right)+h^{2} \\
& g=0.707\left(k^{2}-1\right) \\
& \frac{E(45,45)}{S}=\left[\frac{1}{2}\left(1+k^{2}\right) u^{2}+\left(\frac{1}{4}\left(1+k^{2}\right)+\frac{1}{2} h^{2}\right) v^{2}+\left(\frac{1}{4}\left(1+k^{2}\right)+\frac{1}{2} h^{2}\right) w^{2}\right. \\
&+0.707\left(1-k^{2}\right) u v+\left(-\frac{1}{2}\left(1+k^{2}\right)+h^{2}\right) v w+\left(0.707\left(k^{2}-1\right)\right) u w w^{\frac{1}{2}}
\end{aligned}
$$

or for 2-D flow;

$$
\frac{\bar{E}(45,45)}{S}=\left[\frac{1}{2}\left(1+k^{2}\right) u^{2}+\left(\frac{1}{4}\left(1+k^{2}\right)+\frac{1}{2} h^{2}\right) v^{2}+\left(0.707\left(1-k^{2}\right)\right) u v\right]^{\frac{1}{2}}
$$

From equation 4.10;

$$
\begin{aligned}
a_{1} & =\left(1+k^{2}\right) u+0.707\left(1-k^{2}\right) v+0.707\left(k^{2}-1\right) w \\
b_{1} & =\left(\frac{1}{2}\left(1+k^{2}\right)+h^{2}\right) v+\left(h^{2}-\frac{1}{2}\left(1+k^{2}\right)\right) w+0.707\left(1-k^{2}\right) u \\
c_{1} & =\left(\frac{1}{2}\left(1+k^{2}\right)+h^{2}\right) w+0.707\left(k^{2}-1\right) u f+\left(h^{2}-\frac{1}{2}\left(1+k^{2}\right)\right) v \\
\frac{e^{2}(45,45)}{S^{2}} & =\frac{1}{4 Q^{2}}\left[a_{1}^{2} \overline{u^{\prime 2}}+b_{1}^{2} \overline{v^{\prime 2}}+c_{1}^{2} \overline{w^{\prime 2}}+2 a_{1} b_{1} \overline{u^{\prime} v^{7}}+2 a_{1} c_{1} \overline{u^{\prime} w^{\prime}}+2 b_{1} c_{1} \overline{v^{\prime} w^{\prime}}\right]
\end{aligned}
$$

or for 2-D flow;

$$
\frac{\overline{e^{2}}(45,45)}{S^{2}}=\frac{1}{4 Q^{2}}\left[a_{1}^{2} \overline{u^{\prime 2}}+b_{1}^{2} \overline{v^{\prime 2}}+2 a_{1} b_{1} \overline{u^{\prime} v^{\prime}}\right]
$$


Position $6\left(\psi=0^{\prime 2}, \alpha=0^{\prime}\right)$ normal probe

From equation 8 ;

37
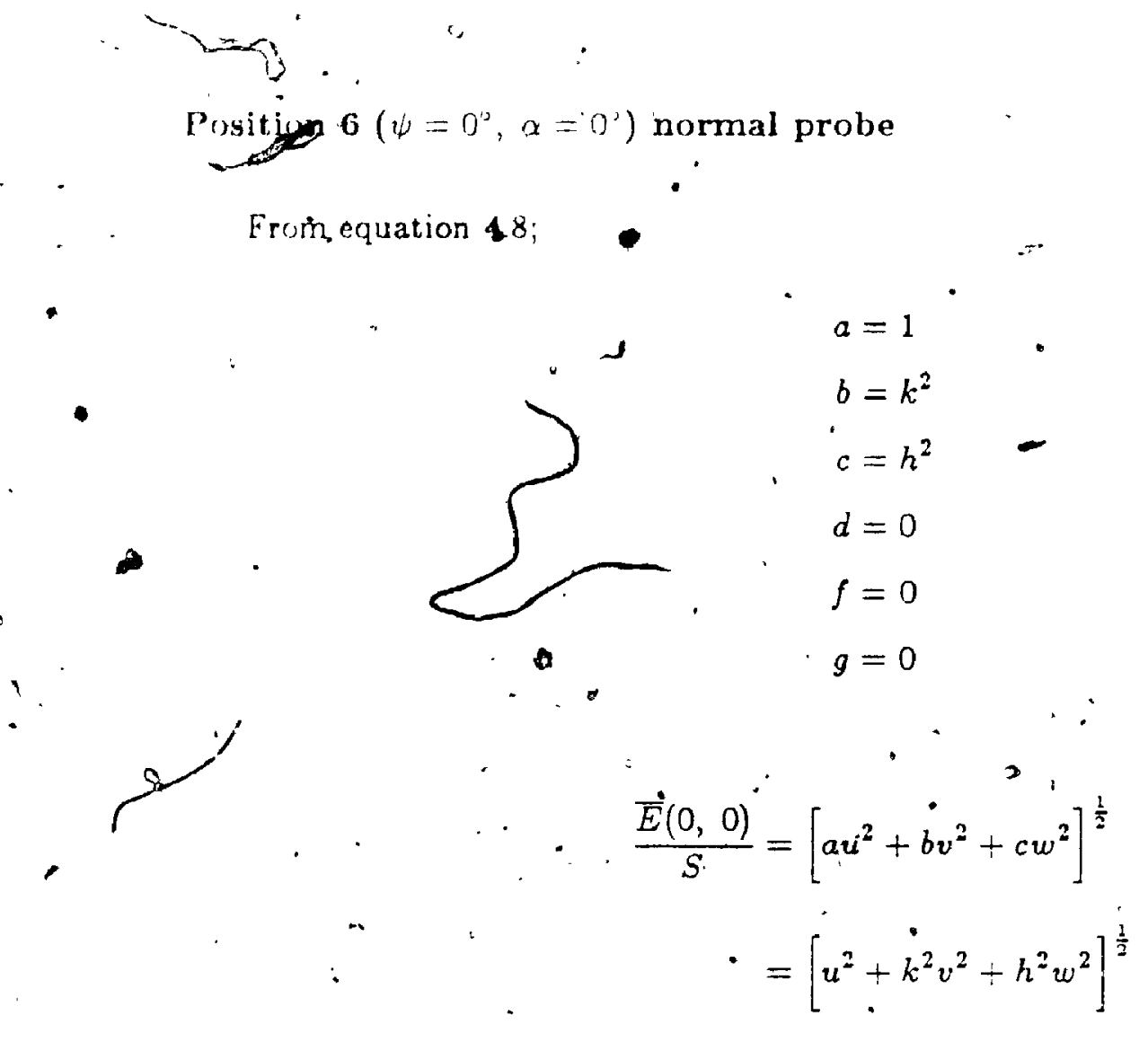

or for 2-D flow;

$\quad \frac{E(0,0)}{S}=\left[u^{2}+k^{2} v_{0}^{2}\right]^{\frac{1}{2}}$

Fromm equation 4.10;

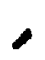

$$
\begin{aligned}
& a_{1}=2 u \\
& b_{1}=2 k^{2} v \\
& c_{1}=2 h^{2} w
\end{aligned}
$$

$$
\frac{\overline{e^{2}}(0,0)}{S^{2}}=\frac{1}{4 Q^{2}}\left[a_{1}^{2} \overline{u^{\prime 2}}+b_{1}^{2} \overline{v^{\prime 2}}+c_{1}^{2} \overline{w^{\prime 2}}+2 a_{1} b_{1} \overline{u_{v^{\prime}}^{\prime}}+2 a_{1} c_{1} \overline{u^{\prime} w^{\prime}}+2 b_{1} c_{1} \overline{v^{\prime} w^{\prime}}\right]
$$

for 2-D flow;

$$
\text { . . } \quad \frac{\overline{e^{2}}(0,0)}{S^{2}}=\frac{1}{4 Q^{2}}\left[a_{1}^{2} \overrightarrow{u^{\prime 2}}+b_{1}^{2} \overline{v^{\prime 2}}+2 a_{1} b_{1} \overline{u^{\prime} v^{\prime}}\right]
$$


Position $7\left(\psi=90^{\circ}, \alpha=0^{\circ}\right)$ normal probe

From equation 4.8;

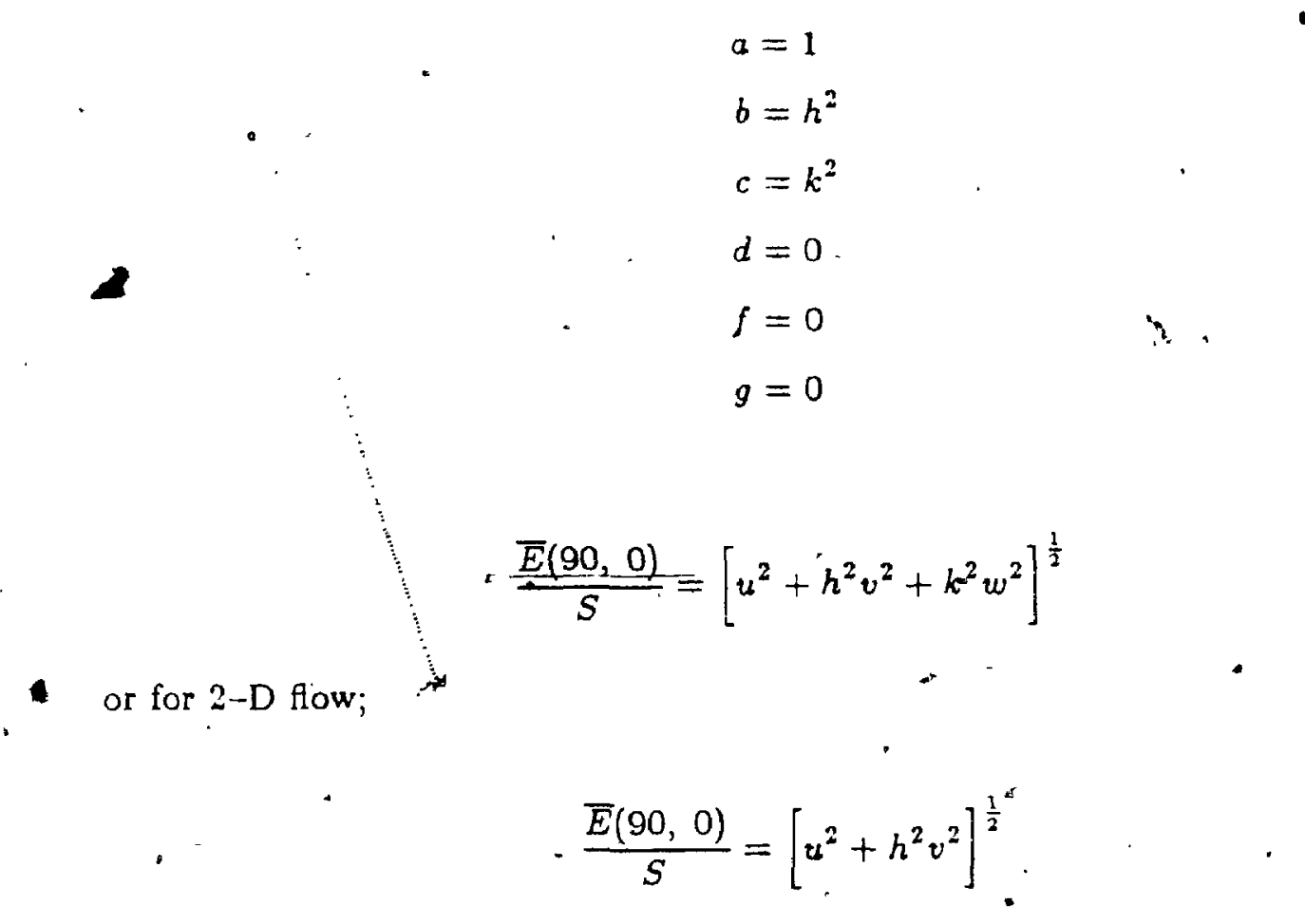

From equation 4.10;

$$
\begin{aligned}
& a_{1}=3 \\
& b_{x}=2 h^{2} v \\
& c_{1}=2 k^{2} w
\end{aligned}
$$

$$
\frac{\overline{e^{2}}(90,0)}{S^{2}}=\frac{1}{4 Q^{2}}\left[a_{1}^{2} \overline{u^{\prime 2}}+b_{1}^{2} \overline{v^{\prime 2}}+c_{1}^{2} \overline{w^{\prime 2}}+2 a_{1} b_{1} \overline{u^{\prime} v^{\prime}}+2 a_{1} c_{1} \overline{u^{\prime} w^{\prime}}+2 b_{1} c \overline{u^{\prime} w^{\prime}}\right]
$$

or for 2-D flo.s;

1. $\quad 2 \frac{\overline{e^{2}}(90,0)}{S^{2}}=\frac{1}{4 Q^{2}}\left[a_{1}^{2} \overline{u^{\prime 2}}+b_{1}^{2} \overline{v^{\prime 2}}+2 a_{1} b_{1} \overline{u^{\prime} v^{\prime}}\right]$

4 


\subsection{Evaluation of the Hot-Fire Signal Analysis}

In this study, an attempt has been made to obtain all mean velocity components and Reynolds stresses, using the analysis pxesented in section 4.2 and data from the wall-jet ipparatus. This also provides a check: of the' analysis.

\section{Mean Velocities:}

The equations derived in section 4.2 for determining the mean velocities $u, v$ and $w_{\text {, }}$. using a slanted probe at positions 1 to 5 , include products of the mean velocities such as $u v, v w$ and $u w$. Thus, six independent equations are needed to solve for the mean velocities at each point of the flow. These six equations can be obtained by a single slanted probe rotated to six different positions or by a combination of slanted and normal probes. Yowakim ${ }^{[55]}$ has concluded that it is preferable to use a slanted probe to obtain 5 equations and a normal probe to obtain the sixth equation. Acrivllelisi ${ }^{1]}$ also uses a normal probe for obtaining a sixth equation.

.The solution of the system of equations in their full form was first tried using an IMSL library routine to solve the matrix, on the University's main computer. The result otained - for $v$ and $w$ were unrealistic. By examining the terms of the equations it was realized that

- addition of equations for positions 1 with 3 and 2 with 4 eliminated the mean velocity products. Thus two equations were obtainged with the unknown terms $u^{2}, v^{2}$ and $w^{2}$ as follows;

$$
\begin{aligned}
\frac{\overline{E^{2}}(0,45)+\overline{E^{2}}(180,45)}{S^{2}}=\left(1+k^{2}\right) u^{2}+\left(1+k^{2}\right) v^{2}+2 h^{2} w^{2} \\
\frac{\overline{E^{2}}(90,45)+\overline{E^{2}}(270,45)}{S^{2}}=\left(1+k^{2}\right) u^{2}+2 h^{2} v^{2}+\left(1+k^{2}\right) w^{2}
\end{aligned}
$$

A third equation can be provided by position 6 using a normal probe as;

$$
\frac{\overline{E^{2}}(0,0)}{S^{2}}=u^{2}+k^{2} v^{2}+h^{2} w^{2}
$$

Solution of the above system of equations also resulted in unrealistic values of $v$ and $w$. Similar problems in determining $v$ and $w$ where encountered by Kelly ${ }^{[34]}$ who used the 
method described by Acrivllelisil and data from the same wall-jet apparafus. The sensitivity of the method in obtaining $v$ and $w$ may be explained as follows; if values vf $v$ and $u$ are small then $\overline{E^{2}}(0,45) \approx \overline{E^{2}}(0,180)$ or $\overline{E^{2}}(0,90) \approx \overline{E^{2}}(0,270)$. When the signals are very

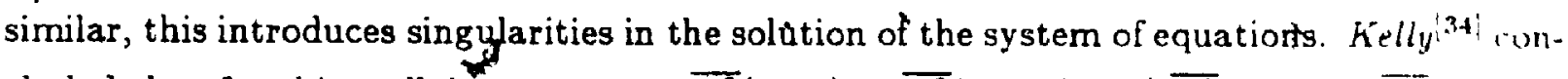
cluded that for this wall-jet apparatus $\overline{E^{2}}(0,45) \approx \overline{E^{2}}(0,180)$ and $\overline{E^{2}}(0.90) \approx \overline{E^{2}}(0,270)$. was indeed the case. Since the flow in the wall-jet achieved good two-dimemsniluliti (Sthanthiran $\left.{ }^{[50]}\right), w$ was dropped from equations 4.11 and 4.12 , and the efforts w.... rected towards the determination of $v$ alone.

Adding equation 4.11 to 4.12 we obtain;

$$
\frac{\overline{E^{2}}(0,45)+\overline{E^{2}}(180,45)+\overline{E^{2}}(90,45)+\overline{E^{2}}(270,45)}{S^{2}}=2\left(1+k^{2}\right) u^{2}+\left(2 h^{2}+\left(1-k^{2}\right)\right):^{2}
$$

Equation 4.14 and equation 4.11 (with $w=0$ ) were solved by an iterative. process as outlined below. First, $v^{2}$ was assumed to be zero and equation 4.14 was solved for $u^{2}$. Usingr this value of $u^{2}, v^{2}$ was calculated using equation 4.11 . The iterations were repeated until the values of $u^{2}$ and $v^{2}$ converged (after about twenty iterations). The values of $v$ obtained by this approach showed a large scatter and they were about ten percent of the values of $u$. All calculations were performed using a computer program, "TEST" shown in Appendix E. If $v$ and $w$ are eliminated from the equation for position 6 then;

$$
\frac{\overline{E^{2}}(0,0)}{S^{2}}=u^{2}
$$

Equation 4.15 provides a check on the analysis. Although $v$ and $w$ were not determued accurately by the analysis, $u$ was within $\pm 3 \%$ of the value obtained by equation 4.15 . Figure 20 shows a good agreersent in the determination of $u$ with a normal and a slanted probe. It is believed that the azalysis can provide more/accurate estimations of $v$ and $w$, if their magnitudes are larger and $\overline{E^{2}}(0,45) \neq \overline{E^{2}}(180,45), \overline{E^{2}}(90,45) \neq \overline{E^{2}}(270,45)$. Due to time limitations, it was not possible to test the analysis further using data from other flow fields.

\section{Reynolds Strésses:}

At first, attempts were made to determine all six components of the Reynolds stresses by solving the system of six equations derived in Section 42 for positions 1 to 6 . The resilts obtained were not realistic mainly because the values of $v$ and $w$ were not determined accurately and they influenced the calculations. Yowakim ${ }^{[55]}$ has found that elimination of $v$ from the Reynolds stress calculations provided better results. 
If the equation for position 3 is subtracted from the equation for position $1, u, \overline{v^{\prime 2}}$ and $\overline{u^{\prime 2}}$, are eliminated giving a direct relationship for the shear stress $\overline{u^{\prime} v}$ as follows;

$$
\begin{aligned}
& \therefore \quad \frac{\overline{e^{2}}(0,45)-\overline{e^{2}}(180,45)}{S^{2}}=\frac{\left(1+k^{2}\right)^{2} u^{2} \overline{u^{\prime 2}}+\left(1-k^{2}\right)^{2} u^{2} \overline{v^{\prime 2}}+2\left(1+k^{2}\right)\left(1-k^{2}\right) u^{2} \overline{u^{\prime} v^{\prime}}}{2\left(1+k^{2}\right) u^{2}} \\
& -\frac{\cdot\left(1+k^{2}\right)^{2} u^{2} u^{\prime^{2}}\left(k^{2}-1\right)^{2} u^{2} \overline{v^{\prime 2}}-2\left(1+k^{2}\right)\left(k^{2}-1\right) u^{2} \overline{u^{\prime} v^{\prime}}}{2\left(1+k^{2}\right) u^{2}} \\
& =2\left(1-k^{2}\right) \overline{u^{\prime} v^{l}} \\
& \overline{u^{\prime} v^{\prime}}=\frac{\overline{e^{2}}(0,45)-\overline{e^{2}}(180,45)}{2\left(1-k^{2}\right) S^{2}}
\end{aligned}
$$

Equation 4.16 is in agreement with that derived by Acrivllelis $[1]$ and Rodi $[47]$. Similarly it can be shown that;

$$
\overline{u^{\prime} w^{\prime}}=\frac{\overline{e^{2}}(90,45)-\overline{e^{2}}(270,45)}{2\left(1-k^{2}\right) S^{2}}
$$

. For $v=w_{1}=0$ the equations for positions 6 and 7 give a direct relation for the longitudinal turbulence, $\overline{u^{\prime 2}}$, as follows;

$$
\frac{\overline{e^{2}}(90,0)}{S^{2}}=\overline{u^{\prime 2}} ; \quad \frac{\overline{e^{2}}(0,0)}{S^{2}}=\overline{u^{\prime 2}}
$$

Equation 4.18 is in agreement with that of Rodi ${ }^{[4]}$ and it provides a check on the method.

Addition of the equations of positions 1 and 3 eliminates $\overline{u^{\prime} v^{\prime}}$ and the resulting equation is in terms, of $\overline{u^{\prime 2}}$ and $\overline{v^{\prime 2}}$. Thus;

$$
\begin{aligned}
& \frac{\overline{e^{2}}(180,45)+\overline{e^{2}}(0,45)}{S^{2}} \\
& =\frac{\left(1+k^{2}\right)^{2} u^{2} \overline{u^{\prime 2}}+\left(1-k^{2}\right)^{2} u^{2} \overline{v^{\prime 2}}+2\left(1+k^{2}\right)\left(1-k^{2}\right) u^{2} \overline{u^{\prime} v^{\prime}}+\left(1+k^{2}\right)^{2} u^{2} \overline{u^{\prime 2}}}{2\left(1+k^{2}\right) u^{2}} \\
& \quad+\frac{\left(k^{2}-1\right)^{2} u^{2} \overline{v^{\prime 2}}+2\left(1+k^{2}\right)\left(k^{2}-1\right) u^{2} \overline{u^{\prime} v^{\prime}}}{2\left(1+k^{2}\right) u^{2}} \\
& =\left(1+k^{2}\right) \overline{u^{\prime 2}}+\frac{\left(1-k^{2}\right)^{2} \overline{v^{\prime 2}}}{\left(1+k^{2}\right)}
\end{aligned}
$$

By substituting equation 4.18 into $4.19, \overline{v^{\prime 2}}$ can be obtained as follows; 


$$
\overline{v^{\prime 2}}=\frac{\left[\overline{e^{2}}(180,45)+\overline{e^{2}}(0,45)\right]\left(1+k^{2}\right)^{2}}{S^{2}\left(1-k^{2}\right)^{2}}-\frac{\left(1-k^{2}\right)^{2} \overline{e^{2}}(90,0)}{S^{2}\left(1-k^{2}\right)^{2}} .
$$

Similarly, addition of the equations for position 1 and 3 eliminates $\overline{u^{\prime} w^{\prime}}$ and the resulting, equation is in terms $\overline{u^{\prime 2}}$ and $\overline{w^{\prime 2}}$. Thus;

$$
\begin{aligned}
& \frac{\overline{e^{2}}(90,45)+\overline{e^{2}}(270,45)}{S^{2}} \\
& \quad=\left(1+k^{2}\right) \overline{u^{\prime 2}}+\frac{\left(k^{2}-1\right)^{2}}{\left(1+k^{2}\right)} \overline{w^{\prime 2}}
\end{aligned}
$$

By substityting equation 4.18 into $4.21, \overline{w^{\prime 2}}$ can be obtained as follows;

$$
\overline{w^{\prime 2}}=\left(1+k^{2}\right) \frac{\left[\overline{e^{2}}(90,45)+\overline{e^{2}}(270,45)\right]}{S^{2}\left(k^{2}-1\right)^{2}}-\frac{\left(-1+k^{2}\right)^{2} \overline{e^{2}}(90,0)}{S^{2}\left(k^{2}-1\right)^{2}}
$$

where " $*$ " indicates values for normal probes.

The Reynolds stresses were calculated according to equations 4.16 to 4.20 using the computer program. "TEST" listed in Appendix E. The results obtained for mean velocity and Reynolds stress components, using this simplified vèrsion of the analysis, are shown in Figures 20 to 26 . The hot-wire signal analysis presented in this thesis has shown sensitivity in calculating $v, w$ and all six Reynolds stress terms when used in its full form. Further evaluation of the analysis-is necessary before any concrete conclusions are made about its accuracy and applicability.

\subsection{Error Analysis • . ".}

\subsubsection{Effects of " $y$ " on Reynolds Stress Calculations.}

As discussed in Section 4.3, $v$ was not included in the Reynolds stress calculationis because it could not be estimated accurately. In this sub-section, an attempt will be madr. to determine the effect of $v$ on the calculation of the shear stress, $\overline{u^{\prime} v}$.

The shear stress, $\overline{u^{\prime} v^{\prime}}$, was first calculated by equation 4.16 , and it was compared $\left.t\right)$ the shear stress, $\overline{u^{\prime} v^{\prime}}$, obtained from the solution of the system of six equations for positions 1 to 6 . In the second method the value of $u$ was that obtained by a single normal probe and $v$ was first assumed to vary linearly with $Y$ as follows; 


$$
v=k \dot{Y}
$$

where $k$ is a constant. For $k=1$ in equation 4.23 the difference, in $\overline{u^{\prime} v^{\prime}}$ obtained by the two methods, increased from $1 \%$ at $Y \approx 0$ to about $10 \%$ at $Y=Y_{m / 2 m}$ and $40 \%$ at $Y>Y_{m / 2}$. When $k=0.1$, the differences in $\overline{u^{\prime} v^{\prime}}$ were $10 \%$ lower as might be expected. It was realized that the assumption of $v=k Y$ was not appropriate and that it introduced substantial differences into calculations of $\overline{u^{\prime} v^{\prime}}$. It was then decided to use the values for $v$ reported by Iruin ${ }^{[32]}$ for Calculation of $\bar{u}^{\prime} v^{2}$ by the six equation analysis. The differences in $\overline{u^{\prime} v^{\prime}}$ values given by the two methods were of the order of $3 \%$ up to the position $Y=Y_{m / 2}$, and increased to about $10 \%$ at $Y>Y_{m / 2}$.

\subsubsection{Effects of Probe Spatial Resolution}

The effect of spatial resolution of the slanted probes on the determination of mean and turbulence quantities was estimated as follows; the $\bar{E}_{b r}$ and $\sqrt{\overline{\bar{e}}_{b r}}$ signals obtained at the point of the maximum shear stress $(Y=0.6 \mathrm{~mm})$, were replaced with those obtained at a distance $\hat{Y}=0.7 \mathrm{~mm}$. The results when compared showed that the change in $u$ was about $1.5 \%, \sqrt{\overline{u^{\prime 2}}}$ changed about $5 \%, \sqrt{\overline{v^{\prime 2}}}$ also changed about $5 \%$ and $\overline{u^{\prime} v^{\prime}}$ changed about $;$ $8 \%$. These results show that spatial resolution has only a small influence on the calculation of the mean velocity, $u$, but substantially: affects the values determined for the Reynolds stresses. Bearing in mind that the $45^{\circ}$ slanted probe spans a distance, $\Delta Y \approx 1 \mathrm{~mm}$ when $\psi=0^{\circ}$ or $180^{\circ}$, the errors on Reynolds stresses due to this effect could easily be of order of $\pm 20 \%$, at the station where these 'measurements were taken.

\subsubsection{Effects of Signal. Sampling Errors}

Although both $\bar{E}_{b r}$ and $\sqrt{e^{2}}$ were sampled with an accuracy of about $0.5 \%$ as described in Section 3.1.2, a test was carried out to determine the errors introduced by signal changes. When $\bar{E}_{b r}$ values were intentionally changed by $0.7 \%$, the mean velocity, $u$; changed by

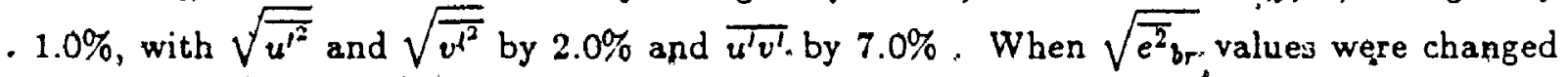
by $1.2 \%, \sqrt{\overline{u^{\prime 2}}}$ and $\sqrt{\overline{v^{\prime 2}}}$ changed by $1 \%$ and $\overline{u^{\prime} v^{\prime}}$ changed by $4.0 \%$.

This test indicates that the Reynolds stress results depend more on changes in the $\bar{E}_{b r}$

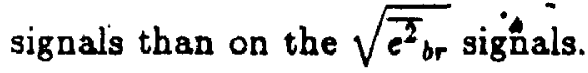

To determine the effect of sampling frequency on the turbulence measurements, the following relation was used to determine the smallest eddy size that could be estimated 
from this sampling technique;

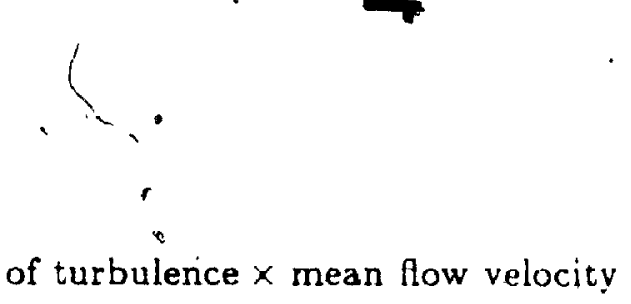

$$
\text { eddy size }=\text { frequency of turbulence } \times \text { mean flow velocity }
$$

For a given sampling frequency, fluctuation frequencies up to one fifth of the sampling, frequency can be measured. For a typical mean flow velocity of about $25 \mathrm{~m} /$ s the eddy size was estimated tc be of the order of one thousand smaller than the distance $Y_{m, 2}$, This indicates, that at the "optimum sampling rate", it is possible to measure almust all $\mathrm{l}_{\mathrm{h}}$. turbulence in the flow. The effect of sampling frequency on the shear stress is not expectul to be large since the contribution of small eddies to shear stress is small ${ }^{\mid 57}$
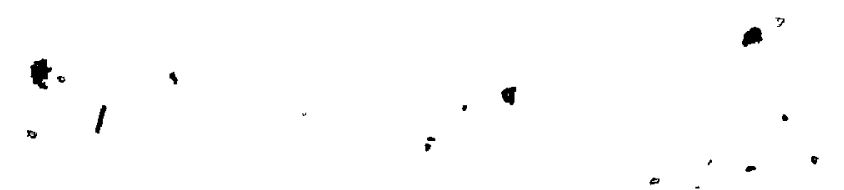


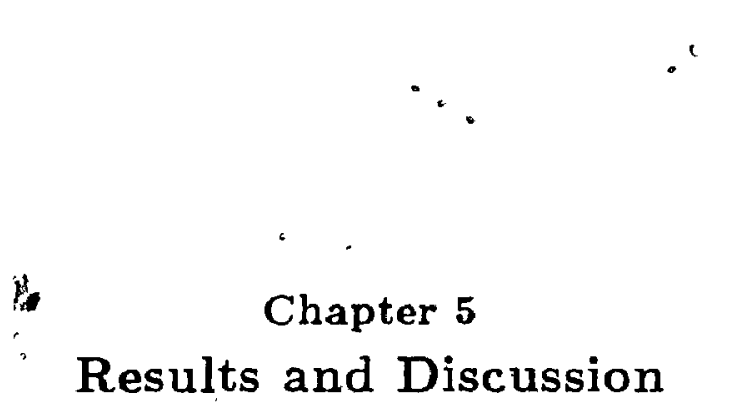

\subsection{Results from the Plane Still Air Wall-Jet Apparatus}

The plane still air wall-jet apparatus results were non-dimensionalized in a similar - fashion as the results of other investigators of wall-jet flows. Figure 20 shows mean velocity profiles obtained with normal and a slanted probe. These profiles are asymmetric with respect to the point of maximum velocity, a result typical of wall-jet flows ${ }^{[30]}$. It is evident from the graph that slanted probes are not suitable for measurements near the walls because the probes must be positioned far enough from the wall to alfow them to be rolled through 360 degrees. As a result, the analysis presented in Section 4.2 is not suitable for near the wall measurements, Figure 21 shows a mean velocity profile obtained with a normal probe at $x / t=93.23$. This profile agrees well with those obtained by Kind and Suthanthiran ${ }^{[39]}$ and Kelly $\left.\right|^{34 !}$ for the same wall-jet apparatus, and that of Irvin $[32]$ obtained for a wall-jet in a moving stream. The agreement of the non-dimensionalized mean velocities obtained at different distances downstream of the wall-jet slot exit indicates that the flow reaches a self preserving state.

Results for longitudinal turbulence obtained at $x / t=93.23$, are plotted in Figure 22 , and agree well with those obtained by Kind and Suthanthiran ${ }^{[39]}$ and Kellyi34]. The longitudinal turbulence is plotted in Figure 23, non-dimensionalized with the maximum mean velocity, $u_{m}$. The curve peaks at the correct disiance from the wall, but has a maximum value of $\sqrt{\overline{u^{\prime 2}}} / u_{m}$, about $15 \%$ higher than the results obtained by Wilson and Goldstein ${ }^{[33\}}$. Kelly ${ }^{\{34}$ ascribes that the difference in the turbulence. intensities may be related to the fact that the wall-jet growth rate for this apparatus is higher than tba conventionally accepted $d Y_{m / 2} / d x$ values of about $0.07[9]$. Figure 26 shows that the results' for the wall-jet growth rate obtgined in this study fall in the same line with the resul is obtained by Kelly [34]. The value of $d Y_{m / 2} / d x$ is about 0.88 which is close to the value of 0.85 obtained by Wilson and Goldstein ${ }^{[53]}$. The fact that the results obtained for the wall-jet growth rate gree with those of Kelly, indicates that $Y_{m / 2}$ values were determined correctly,

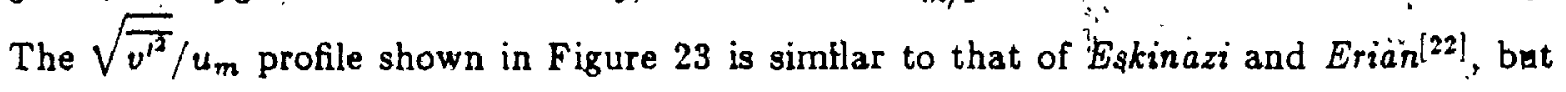




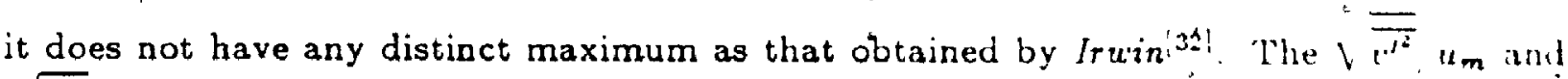
$\sqrt{\overline{u^{\prime 2}}} / u_{m}$ curves meet at a distance of about $Y / Y_{m / 2}=1.6$ as those of $I_{r u z n^{\prime}} 32 !$. -

Figure 25 shows a shear stress profile obtained at $x / t=93.23$ plotted with profiles of other investigators. All plots are in fair agreement in the region below the point of maximum shear stress, but have di.Ferent maxima and drop differently in the region beyond the pum, of maximum shear stress., Launder and. Rodi ${ }^{\{3\}}$ have identified that among the avilianil. sets of data there is a considerable scatter in the shear stress poofiles. In a comparison of th" shear stress profiles of Wilson and Goldstein ${ }^{[5]}$, Guitton [28], Giles et al [26] and Bradshiz:" and $G e e^{[9]}$, they show that the differences in the shear stresses are large at the reigun. beyond the point of maximum shear stress, but they do not discuss this effect. The' fart that the maximum shear stress point obtained in this study does not coincide with thist if Kelly ${ }^{[34}$, may be explained as follows; according to Wilson and Goldstein ${ }^{[53 \mid}$, slot Reynolds number effects alter the viscous dissipation rates of the turbulence affecting the value of the maximum shear stress to about $15 \%$. Thus, differences in the slot Reynolds number achleved in this set of data, with the corresponding slot 'Reynolds number achieved by Kelly ${ }^{[34}$, may have caused the difference in the value of the maximum shear stress. The point of $\overline{u^{\prime} v^{\prime}}=0$, extrapolated from the plot of Figure 25, does not coincide with the point where $d u / d y=0$. A result typical of non-symmetric mean velocity profile flows ${ }^{|30|}$. In this set of data, the point of $\overline{u^{\prime} v^{\prime}}=0$ lies closer to the wall than the point where $d u / d y=0$. The same result has been described by Kelly ${ }^{[34]}$ for the same wall-jet apparatus. Irwin ${ }^{32\}}$, Wilson and Goldstein[53], Eskinazi and Erian[22], Tailland and Mathieut51] and Bequier ${ }^{[4]}$ among others, reported the same result for wall-jet flows. Results obtained for $\overline{u^{\prime} w^{\prime}}$ are" plotted and shown in Figure 24 along with the results of $\overline{u^{\prime} v^{\prime}}$. The fact that $\overline{u^{\prime} w^{\prime}}$ is not zero, as is the case for two-dimensional flows, shows that some flow three-dimensionalities were present.

The results obtained" for the wall-jet apparatus provided confidence for carrying out further measurements for the B.L.C. apparatus. 


\subsection{R.esults from the B.L.C. Apparatus}

\subsubsection{Flow-Quality}

\section{Still Air Measurements: -}

The maximum total pressure distribution of the cylinder jet flow under still air conditions, exhibited random short wave length fluctuations along the span. Kind et al. ${ }^{[38 \mid}$ have reported similar measurements for the same cylinder and they have concluded that these fluctuations in total pressure are an unavoidable consequence of the inferent instability of the flow in the outer region of a wall-jet with convex curvature. Guitton and Newman ${ }^{[29]}$ also report similar fluctuations of the total pressure and they concluded that they are due to slot lip imperfections. The results obtained at $\theta=32^{\circ}, 62^{\circ}$ and $92^{\circ}$ are shown in Figure 27. The cylinder surface static pressure distribution in still surroundings is shown in Figure 28. The results are in good agreement with the results of Fekete $e^{[23]}$ for the region from $\theta=40^{\circ}$ to $110^{\circ}$. The range variation in the pressure near the slot in the present data may be due to slot irregularities. Feketel 23$]$ reports a number of pressure distributions depicting similar variations of the pressure near the slot, when he varied the slot thickness and the Reynolds number. .

It is suspected that the presence of the forebody may be the reason why there is no - pressure drop in the region of $\theta^{\prime}=110^{\circ}$ to $\theta=150^{\circ}$. Furthermore the flow will not separate after $\theta=150^{\circ}$ and pressure dróp will not occur..

\section{'Static Pressure Measurements:}

The static pressure distribution over the cylinder surface and the forebody for $C_{\mu c}=$ $0.1,0.2,0.22 ; C_{f w}=0.11$ and $U_{\infty}=21 \mathrm{~ms}^{-1}$ are shown in Figure 29. The pressure , distributions over the forebody for all $C_{\mu c}$ values show that the forebody design requirement

" "for about zero pressure gradiept near the slot is met. The pressure distribution also shows that at higher $C_{\mu c}$ valures, the flow is accelerated around the cylinder and forebody because of an increase in the circulation around the forebody. At the injection slot the flow is reenergized and it separates at $\theta=62^{\circ}$ for $C_{\mu c}=0.2,0.22$. It also evident from the graph that the pressure distributions for $C_{\mu c}=0.2,0.22$ are very similar. This indicates that relatively small changer of $C_{\mu c}$ do not influence the flowfield significantly. For $C_{\mu c}=0$, the flow separated at about $\theta=32^{\circ}$. 
Static Pressure Distribution on the Plane Wall:

The static pressure distribution obtained at the $x=7$ in and $x=11$ in statuons downstream of the slot in the plane wall-jet and at sfanwise distances of $z=-7$ in to $z=+7$ in are shown in Figure 30 for $C_{\mu c}=0.1,0.22$. Results show that the flow on the rear wall is accelerated near the ceiling and the floor, and it is two-dimensionial uver about the central six inches. At $x=11$ in, the conditions are more severe as the thw approaches separation. This suggests that the flow over the side wall does not achire............. good two-dimensionality as that reported by Gooden ${ }^{[27]}$. His results have shown that f1: $C_{\mu c}=0.3, C_{\mu w}=0.0$ and with suction on, the spanwise pressure distribution variend i., about $2 \%$. In the present study, it is evident that the presence of the forebody increas's the bound vorticity which is shed downstream, introducing flow deterioration. Moreover, the suction is not adequate to completely elimirate three-dimensional effects in the region away from the cylinder.

Flow Visualization:

The effect of end-wall suction is siown in six photographs taken of the tuft study; for $C_{\mu c}=0.1,0.2,0.22$, shown in Figures 31,32 and 33. The tufts show clearly that endwall suction improves the flow two-dimensionality on the cylinder and on the wall. For $C_{\mu c}=0.1$, the tufts show best alignment since the shedding yorticity is less for lower $C_{\mu c}$ values. The tufts show that the flow is two-dimensional for $x \leq 7$ in on the plane wall.

The surface flow visualization study results are shown in Figures 34 to 37 . It is evident from the photographs that the point of separation is not uniform spanwise. For $C_{\mu c}=0.1$, the separation point spanwise uniformity is best. This visuatization scheme suggests that the flow away from the mid-span region has higher momentum and it separates later than the flow in the mid-span. Gravity effects have certainly influenced the quality of the surfacr visualization study.

Mean Velocity Profile Measurements:

Mean velocity profiles downstream of the cylinder slot exit are shown in Figures 38 to 40 , for three different spanwise stations. These profiles show fair agreement for $\widetilde{C}_{\mu c}=0.1,0.22$ and at stations close to the separation point, more departure from two-dimensionality is evident. At the separation point, the profiles show a large departure from two-dimensionality. Figure 39 indicates reasonable two-dimensionality for a span of about 6.7 in $(17 \mathrm{~cm})$ : Further away from mid-span, the profiles are distinctly fuller. This agrees with the surface" 
visualization study which showed that, separation occurs somewhat later at the region away from the mid-span.

The flow quality checks have demonstrated that the flow is two-dimensional for about . 6.7 in in the mid-span of the cylinder. The nature of the flow is such that it is difficult to obtain two-dimensional flow conditions at the cylinder separation region. Improvements of the two-dimensional characteristics of the flow are desirable.

\section{Temperature Effects}

The temperature difference between the cylinder jet air and the freestream air, measured using the cold-wire method described in Section 3.2.4 and the approximation described by equation 3.16, was no more than $6^{\circ} C \pm 8 \%$ for the most severe conditions of $C_{\mu c}=0.22$ at $\theta=2^{\circ}$ and at $Y$ of about $1 \mathrm{~mm}$. The temperature differences away from the cylinder wall and at all downstream measuring stations $\left(\theta>2^{\circ}\right)$ were less than $6^{\circ}$.

The fact that the temperatures measured were lower than the supply air temperature, measured at the inlet of the cylinder plenum, may be due to the rapid mixing of the cylinder wall-jet air with the freestream air. An estimate of the maximum percentage error introduced by temperature variation is about $4 \%$ based on the following relationship $[56]$. (see nomenclature, Section 3.2.4);

$$
\% \text { error }=\frac{2 \Delta T}{T_{s}-T_{e}} \times 100
$$

\subsubsection{Mean Velocity and Reynolds Stress Measurements}

The mean velocity component, $u$, and the Reynolds stresses in this study have been calculated according to the analysis described in Chapter 4 , using equations 4.15 to 4.21 . All the data were transfered to the University's main frame computer system for processing.' The computer program, DATARED, shown in Appendix D gas developed to perform the calculations and another program, SEP, was developed to prepare separate files for plotting of the results.

\section{Mean Velocities:}

The mean velocity profiles measured at seyen stations downstroam of the cylinder slot exit for $C_{\mu c}=0.1$ and at eight stations fof $C_{\mu c}=0.2,0.22$ are presented in Figures 41,42 and 43 respectively. The flow downstrdam of the cylinder slot does not achieve a 
self preserving state because the ratio of the maximum velocity, $u_{m}$, to the velocity of the external flow, $u_{e}$, varies along the flow. The mean velocity profiles are presented in a similar fashion to those of Kind et al. ${ }^{[38]}$. Mean velocity results at several measuring stations and at different cylinder momentum coefficients are tabylatinppendix G.

For all cylinder momentum coefficients and at $\theta=2^{\circ}, 12^{\circ}, 22^{\circ}$ and $32^{\circ}$, the sheak lityrer was not thick enough to enable measurements inside the inner layer, while from $\theta$ " 12 onward more points in the inner layer could be obtained. All mean velocity profiles sink: distinct maxima and minima until $\theta^{\circ}=52^{\circ}$ for $C_{\mu c}=0.2,0.22$. The velocity maxima anl minima tend to disappear as the shear layer approaches separation. The resultung shr.sr layers are similar to conventional turbulent boundary layers. The mean velocity protiles have a flatter maximum point region than the the profiles obtained in the plane-wall jet apparatus. Dvorak ${ }^{[17]}$ explains that mean velocities profiles can exhibit both a maximum and minimum because the injected fluid cannot immediately entrain the entire upstream boundary layer fluid. The mean velocity results show that the ininged fluid loses its momentum as it flows downstream of the cylinder wall, due to wall viscous effects and due to mixing with the slower moving external flow. In wall-jet flows over convex surfaces, such as the present; the outer flow is unstable causing rapid mixing.of the jet fluid with the external flow ${ }^{[35]}$. This results in a rapid decay of the velocity maximum and increase the growth rate of the wall-jet. Giles et al. ${ }^{[36]}$, Guitton and Newman ${ }^{[29]}$, Wilson"and Goldstein ${ }^{[53]}$ and Kind et al. ${ }^{[38]}$ have demonstrated that streamwise curvature has a large effect on gfowth rate of wall-jets: This effect, although desiable, because the velocity minimum does not persist, deepens and promotes separation as hllustrated in Figure 4, results in a relatively rapid decaying of the maximum velocity. With rapidly decaying maximum velocity of the walljet, the flow separates relatively early from the cylinder surface. The foint of separation occirs at about $\theta=62^{\circ}$ for $C_{\mu c}=0.1$ and at about $\theta=72^{\circ}$ for $C_{\mu \mathrm{t}}^{\prime}=0.2,0.22$.

The mean velocity profiles measured with $C_{\mu c}=0.22$ are quite similar to those obtainerd at $C_{\mu c}=0.2$. The comparison shows that small $C_{\mu}$ changes only affect the flow in the region close to separation. At upstream station $\$$, the flow is almost unaffected by small changes in $C_{\mu c}$. The small differences in the mean velocity measurements at $C_{\mu c}=0.2,0.22$ also. indicate that consistent results may be obtained from present B.L.C. apparatus with good repeatability. Figure 44 shows that for $C_{\mu c}$ at $\theta-12^{\circ}$, the variation of $C_{\mu w}$ has very little effect on the cylinder mean velocity profile. This, result agrees with $\mathscr{G}_{\text {ooden }} n^{27 \mid}$ who concluded that the variation of $C_{\mu \omega}{ }^{\circ}$ did not have significant effects on the mean velocity profiles ineasured on the cylinder; and external flow is essentially the same for different $C_{\mu w}$ 
vitues.

The results obtained in this study cannot be compared in detail to results reported by Kind et al. ${ }^{38 !}$, because the flow conditions and geometry are different in the present study, i.e. the angular position of the- linder jet plane with respect to the mean flow direction is $90^{\circ}$ in this study and the couresponding angular position was $32^{\circ}$ in their ${ }^{[38]}$ st Morenver, the presence of the forebody has introduced more differences in the flowfield.

\subsection{Disçussion.}
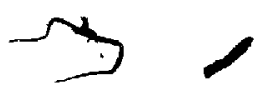

\subsubsection{Reynolds Normal Stresses}

The normal Reynolds stress profiles. measured at seven stations downstream of the cylinder slot exit for $C_{\mu c}=0.1$ and at eight stations for $C_{\mu c}=0.2$ and 0.22 are presented in Figures 45 to 54 . The maximum mean velocity, $u_{m}$, has been used as a non-dimensionalizing velocitymarameter while the distance from the wall, $Y$, has been kept dimensional, as in the mean velocity results.

At the measuring station of $\theta=2^{\circ}$, results for $\overline{v^{\prime 2}}$ were not obtained because the thickness of the jet is very small and it was not possible to obtain results for the jet flow region using the slanted probe. The normal Reynolds stress, $\sqrt{v^{\prime 2}} / u_{m}$, profiles presented here shọw significant differences from those obtained in the plane wall-jet apparatus. The $\sqrt{\overline{v^{\prime 2}}} / u_{m}$ profiles presented here are significantly larger than that obtained in the plane walljet apparatus. Esk ${ }_{2} i$ and $Y_{e h} h^{[21]}$ state that the wall curvature infuences the turbulence quantities to a significant extent. Moreover, Guitton and Newman ${ }^{299}$ have concluded that wall curvature dramatically increases the turbulence component, $\overline{v^{\prime 2}}$, as compared with $\overline{u^{\prime 2}}$. This may be explained in terms of the instability to radial fluctuations of the flow in the outer region of wall-jets having convex currature.

The turbulence intensity, $\sqrt{v^{\prime 2}} / u_{m}$ is larger thap the longitudinal turbulence intensity, $\sqrt{u^{12}} / u_{m}$ in the shear layer at all measaring stations upstream of the separation point and at all cylinder momentum coefficients. Both $\sqrt{\overline{v^{12}}} / u_{m}$ and $\sqrt{\overline{u^{12}}} / u_{m}$ intensities become progressively larger as the flow develops downstream of the slot, because the mean velocity is decreasing in the adverse pressure gradient downstream of the slot. Wilson and Goldstein ${ }^{[53]}$ " also report $\sqrt{v^{12}} / u_{m}$ intensities larger than $\sqrt{u^{12}} / u_{m}$ in a wall-jet over a circular cylinder flow and Guitton and Newman ${ }^{[29]}$ report some deviation in $\sqrt{v^{\prime 2}} / u_{m}$ and $\sqrt{u^{\prime 2}} / u_{m}$ for a jet over logarithmic spiral surfaces. 


\section{.}

At stations $\theta=2^{\circ}, 12^{\circ}, 22^{\circ}$ and $32^{\circ}$, both turbulencé intensities $v_{u^{\prime 2}} u_{m}$ and $v \overline{u^{\prime 2}}$ are very similar for all $C_{\mu c}$ values. At measuring stations close to the separation point, th: turbulence intensities depend significantly on the $C_{\mu c}$ value. Thus, at $C_{\mu c}=0.1$ and at $\theta=42^{\circ}, 52^{\circ}, 62^{\circ}$, the turbulence intensities are considerably larger than the "rrresponding intensities for $C_{\mu c}=0.2$ and 0.22 . The turbulence intensities, $v u^{\prime 2}, u_{n \rightarrow}^{-}$ami $\sqrt{\overline{v^{\prime 2}}} / u_{m}$ obtained for $C_{\mu c}=0.2,0.22$ are in good agreement at the measuring stalus: $\theta=12^{\circ}, 22^{\circ}, 32^{\circ}$ and $42^{\circ}$. At $\theta=52^{\circ}, \sqrt{\overline{u^{\prime 2}}} / u_{n}$ profiles, are similar while the $、 v^{\prime \prime}$.. profile for $C_{\mu c}=0.22$ is slightly larger. At $\theta=62^{\circ}$, it is shown clearer that $v \overline{\overline{v^{\prime 2}}}$, "m $m 1 \cdot n$ sity is larger for $C_{\mu c}=0.22$. This indicates that at stations close to the flow separaum, ih, turbulence intensities are more reliant on small variations of $C_{\mu c}$. The $v \overline{u^{t 2}} / u_{m}$ inturuspu....

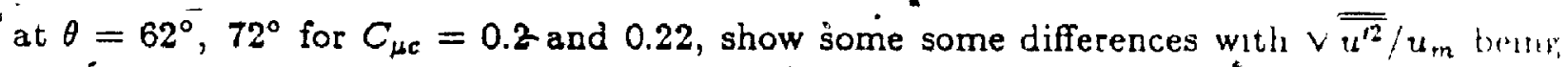
lower at $C_{\mu c}=0.22$, presumably because the flow is slightly farther from the separiaun The turbulence intensity profiles do not show any large differences in the region outside the shear layer at measuring stations before the separation point. The turbulence intensities, $\sqrt{\sqrt{u^{12}}} / \mu u_{m}$ and $\sqrt{v^{12}} / u_{m}$ of the external flow for $C_{\mu c}=0.1$ reaches a congtant value of $1.5 \%$. at $\theta=2^{\circ}, 1.9 \%$ at $\theta=22^{\circ}, 2.8 \%$ at $\theta=42^{\circ}$ and $15 \%$ at the separatjon point of $\theta=62^{\circ}$ When $C_{\mu c}$ is increased to 0.2 , the corresponding intensities are lower, i.e. $1.3 \%$ at $\theta=2^{\circ}$, $1.6 \%$ at $\theta=22^{\circ}$ and about $10 \%$ at the separation point. The large increase in the turbulence intensities in the region close to separation may reflect fluctuation of the external inviscid flow. The maxima of the turbulence intensity profiles occur at approximately the,

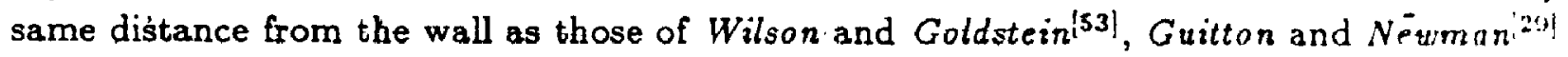
and Irwin ${ }^{[32]}$.

Quantitatively, the results for the turbulence intensities are fower than thrse avialabli: in the literature for wall-jets over convex surfaces in still air surroundings. This may be due to the fact that mixing is less vigfous in wall-jet flows with the external flow, than in wall-jet flows in still surroundings.

Figures 52, 53 and 54 show results of $\sqrt{\overline{u^{\prime 2}}} / u_{m}, \sqrt{\overline{v^{\prime 2}}} / u_{m}$ and $\sqrt{\overline{w^{\prime 2}}} / u_{m}$ for thrie different flow conditions. These results show large differences between $\overline{u^{\prime 2}}$ and $\overline{v^{\prime 2}},\left.\overline{w^{\prime 2}}\right|_{w h l}$, the differences between $\overline{v^{\prime 2}}$ and $\overline{w^{\prime 2}}$ are smaller. The deviation of these results is attributed partly to three-dimensional effects and partly to inaccuracies in the measuremerits The larger deviation of $\overline{u^{\prime 2}}$ from $\overline{v^{\prime 2}}$ and $\overline{w^{\prime 2}}$ may be due to the fact that $\overline{v^{\prime 2}}$ and $\overline{w^{\prime 2}}$ were calculated using signals from both a normal and a slanted probe, according to equations 4.20, 4.21 of Chapter 4 whereas $\overline{u^{\prime 2}}$ was measured with a normal probe. Moreover, spatial resolution effects are more pronounced when a slanted probe is used, because the probe 
was perpendicular to the wall as shown in Figure 18.

The results presented in Figures 52,53 and 54 indicate that $\overline{u^{\prime 2}}, \overline{v^{\prime 2}}$ and $\overline{w^{\prime 2}}$ are approxamately equal,indicating that the turbulence is definitely not isotropic. The longitudinal turbulence profile, $\sqrt{\overline{u^{12}}} / u$, obtained at $\theta=12^{\circ}, C_{\mu c}=0.1$ and at $C_{\mu w}=0.11$ and 0.2 are presented in Figure 50. These results show that variation of $C_{\mu \nu}$ does not affect the urbulence intensities downstream of the cylinder slot exit, as was the case for the mean valocity profiles discussed previously.

Tabulated results for both $\sqrt{\overline{u^{\prime 2}}} / u_{m}$ and $\sqrt{\overline{v^{\prime 2}}} / u_{m}$ obtained in this study are included in Appendix G.

\section{5,3.2 Reynolds Shear Stresses}

The Reynolds shear stress profiles measured at six stations downstream of the cylinder slot exit for $C_{\mu c}=0.1$ and at seven stations for $C_{\mu c}=0.2,0.22$ are presented in Figures 55 to 60 . The square of the maximum velocity, $u_{m}^{2}$, has been used as a non-dimensionalizing parameter for the shear stresses and $Y$ has been kept dimensional as in the turbulence and mean velocity graphs. Shear stresś profiles fơr all $C_{\mu c}$ values show no significant scatter between successive measuring points at all measuring stations upstream of the separation point. This is because the turbulence intensities before the point of separation were small, thus, it is expected that the hot-wire signals are sampled more accurately. At $\theta=52^{\circ}, 62^{\circ}$ for $C_{\mu c}=0.1$ and at $\theta=62^{\circ}+72^{\circ}$ for $C_{\mu c}=0.2,0.22$, the scatter 'in the data increased significantly. It is evident from the graphs that it was not possible to obtain many data points in the negative shear stress region near the wall, especially at measuring stations. close to the slot exit. This is because the shear layer being very thin, did not allow for measurements close to the wall using a slanted probe. More points could be obtained in the negative shear layer at measuring stations downstream of the slot exit, because the shear layer progressively thickened farther away from the slot exit.

- The shęar stress profiles obtajned, for $C_{\mu c}=0.2,0.22$ are in fair agreement with each other. At $\theta=12^{\circ}$ to $52^{\circ}$, the profiles for $C_{\mu c}=0.2$ and 0.22 are virtually the same, with slight differences in their maxima. As the separation point is approached at measuring stations of $\theta=62^{\circ}, 72^{\circ}$, the two profiles show some differences. Thus, at $\theta=62^{\circ}$ for $C_{\mu c}=0.22$ the shear stress profiles has a larger maximum than the profile of $C_{\mu c}=0.2$. The profile for $C_{\mu c}=0.2$ shows that the shear stress is close to zero near the wall, indicating that the now separates earlier for $C_{\mu c}=0.2$ than f.rr $C_{\mu c}=0.22$. At $\theta=72^{\circ}$, the profiles 
for $C_{\mu c}=0.2$ and $C_{\mu c}=0.22$ both show a significant increase in shear stress some distance from the wall. As expected, the values near the wall are fairly close to zero.

The shear stress profiles obtained for $C_{\mu c}=0.1$ are similar to those obtained for $C_{\mu c}=0.2$ with the profiles for $C_{\mu c} \doteq 0.2$ having slightly higher maxima, at measurung stations of $\theta=12^{\circ}$ to $42^{\circ}$. At $\theta=52^{\circ}, 62^{\circ}$, the shear stress profiles for $C_{\mu L^{\circ}}-01.02$ i $2 r^{\prime \prime}$ considerably different because the flow separates earlier at lower $C_{\mu c}$ values. . Ill the whe.u

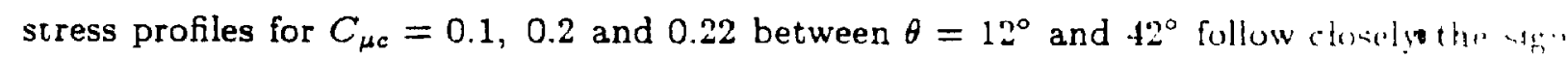
of the meapvelocity gradient as expected. Thus, the shear stresses cross the $z$. $r 6$, wis is

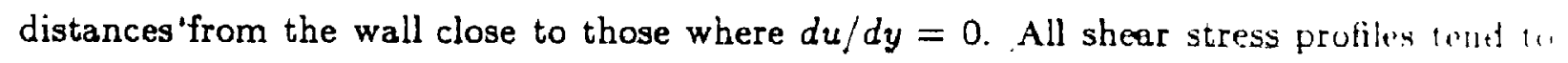
follow the same general trend which can be described as follows.

The profiles cross the zero axis at approximately the same point where $u=u_{\text {mux }}$, they are positive for the wall-jet flow region. They cross the zero axis again at the point. where $u \approx u_{\min }$ and have a short negative shear stress region. The zero axis is again crossed at - approximately the point of $d u / d y=0$ for the external flow. This can be seen better in profiles for $C_{\mu c}=0.1$ at $\theta=12^{\circ}, 22^{\circ}$ and for $C_{\mu c}=0.2$ at $\theta=12^{\circ}$. The points were. $\overline{u^{\prime} v^{\prime}}=0$ lie further from the surface than the points where $d u / d y=0$. Bequier $|4|$ also demonstrates that the shear stress profiles follow closely the sign of $\dot{d}^{\prime} / d y$ for both the shear layer and the external flow region, but in his case the positions of $\overline{u^{\prime} v^{\prime}}=0$ lie closer to the wall with respect to the positions of $d u / d y=0$. Erian ${ }^{|20|}$ describes that $\overline{u^{\prime} v^{\prime}}=0$ does net necessarily occur closer to the wall with respect to the position of $d u / d y=0$ and has demonstrated experimentally that for wall-jet flows which experience adverse pressure gradients, the location of $\overline{u^{\prime} v^{\prime}}=0$ is further from the wall with respect to the $d u / d y=0$ position. The present flow experiences a strong streamwise curvature and this mak explain the fact that $\overline{u^{\prime} v^{\prime}}=0$ lies further from the wall with respect to the $d u / d y=0$ position.

As the wall-jet gradually loses its momentum, the $\overline{u^{\prime} \nu^{\prime}}=0$ points lie considerably. "further from the wall with respect to the positions of $d u / d y=0$. Eskinazi and Erian ${ }^{22 \mid}$ refer to the region between, $\overline{u^{\prime} v^{\prime}}=0$ and $d \dot{u} / \dot{d y}=0$ as areas of negative turbulence production or zones of opposing shear. Hinze $e^{[30]}$ explains that in these regions $\overline{u^{\prime} u^{\prime}}$ and $d u / d y$ have opposite signs and their product yields a negative contribution to the rate of change of the kinetic energy of the turbulence. The negative contribution does not necessarily imply a gain of energy by the mean motion at the cost of the energy of the turbulence.

Figures 61,62 and 63 show measurements of $\overline{u^{\prime} w^{\prime}}$ for $C_{\mu c}=0.1$ at $\theta=32^{\circ}, 42^{\circ}$ and for $C_{\mu c}=0.2$ at $\theta=42^{\circ}$ together with the corresponding measurements of $\overline{u^{\prime} v^{\prime}}$. All profiles 
Show that $\overline{u^{\prime} w^{\prime}}$ is close to zero outside the shear flow. Inside the shear flow region, the measurements give non-zero values for $\overline{u^{\prime} w^{\prime}}$; this indicates imperfect two-dimensionality of the flow. Note however that the $\overline{u^{\prime} w^{\prime}}$ values are substantially less than the $\overline{u^{\prime} v^{\prime}}$ values at corresponding positions in the shear flow.

Figure 64 shows shear sțress profiles obtained in the present study, non-dimensionalized with $u_{m}^{2}$ and $Y$ with $Y_{1 / 2 m}$, (see Nomenclature in Figure 5). These non-dimensionalized shear stress profiles do not show a good agreement, for $C_{\mu c}=0.2$ at $\theta=22^{\circ}$ and for $C_{\mu c}=0.1,0.2$ for $\theta=42^{\circ}$. This may be explained by the fact that the flow is not reaching,

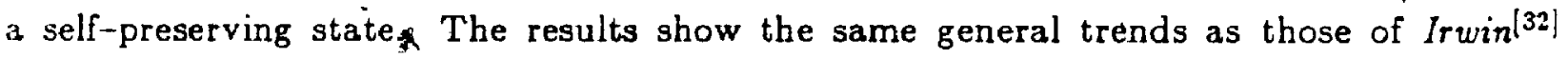
for a wall-jet in a freestream and those of Wilson and Goldstein [53] in a plane wall-jet flow in still air surroundings. The results for the shear stresses obtained in this study, although having demonstrated the same trends as those of previous works, are subjected to errors due to probe spatial resolution. Errors due to probe spatial resolution were unavoidable in the present flow because the shear layer is thin. Certainly more information is needed to assess the magnitude of the errors introduced by the probe spatial resolution effects in the measurements of the shear stresses. Due to the time limitations, it was not possible to investigate further the effect of the probe spatial resolution on the shear stress measurements.

\subsection{Conclusions}

The hot-wire signal analysis derived in this study has shown sensitivity in calculating the mean velocity components $v, w$ in a plane wall-jet flow in still surroundings. Its derivation involves the assumption of turbulence levels of equal to or less than twenty percent. The sensitivity of the analysis in calculating $v, w$ has forced simplifications on the analysis in the calculation of the Reynolds stresses. The analysis in itomplified form agrees with the analyses of other investigators. Further testing of the analysis is necessary before concrete concllusions are drawn about its applicability. The analysis when tested with data for a plane wall-jet flow has produced similar results with those of other investigators.

The modifications designed for the B.L.C. apparatus have produced a thicker bound-, ary layer downstream of the cylinder slot, which facilitates measurements of both mean velocities and Reynolds stresses. The measurements of $\overline{v^{\prime 2}}$ and $\overline{u^{\prime} v^{\prime}}$ are subjected to probe spatial resolution errors because the probe is oriented normal to the surface of the cylinder. In the mean velocity and longitudinal turbulence data, probe spatial resolution effects are 
not significant because the probe was oriented parallel to the surface of the cylinder The flow quality experiments have shown that the flow two-dimensionality has been affected by the presence of the forebody and improvements are desirable. The flow quality experiments have shown that the flow is two-dimensional for the central 6.7 in of the cylinder spin and $18 \mathrm{~cm}$ span of the plane wall downstream of the plane wall-jet. The flow also shows a spanwise non-uniform separation.

Temperature effects have not been considered in the analysis of the result:s. 1)..inlle the errors that may have been introduced in the results, it has been demonstrated thist repeatable measurements can be obtained for the B.L.C. apparatus. Wean velocty and Reynolds stress results are insensitive to small cylinder momentum coefficient changes at stations close to the slot exit, but are sensitive at stations close to the separation. 'Th' shear stress results follow the same trends as those of other investigators and compare well with wall-jet results available in the literature.

So far as the author is aware, the present experiment provides the first mean velocity and Reynolds stress data for different momentum coefficients, for cases where flows consisting of a wall-jet mixing with an upstream boundary layér in a strong adverse pressure gradient, develop into a conventional boundary layer. The results presented in this work, provide data for an important and complex flow, which can be used in testing of prediction methods.

\subsection{Recommendations for Future Work}

One can easily check the derivation of the analysis by following the step by step detailed derivation included in Appendix F. The analysis may be tested in its full form with results obtained from the simplified equations, 4.15 to 4.21 . The computer program, TFST, listed in Appendix $\mathrm{E}$ can be followed for these comparisons.

Uniless the mean velocities, $v, w$, can be computed and checked, they should not be included in the Reynolds stress calculations because they introduce large errors. The now quality in the B.L.C. apparatus may be improved to some extent if more suction is applied through the porous end plates. With the forebody already mounted in the B.L.C. apparatus and by using the $A D A C S$ computer programs listed in Appendix D, it is a simple matter to proceed with further experimentation. The results obtained in this study may be compared to similar computational schemes as those of Kind et al. ${ }^{[38\}}$. 


\section{References}

1 ACRIVLELLIS, M.

2. ACRIVLELLIS, M.

3. ACRIVLELLIS, M.

4. BEGUIER, C.

5. BRADSHAW, P. .

6. BRADSHAW, P.

7. BRADSHAW, P.

8. BRADSHAW, P.

9. BRADSHAW, $P$. GEE, M.T.

10. BRITISH STANDARD 1042
An Improved Method for Determining the Flowfield of Multi-dimensional Flows of any Turbulence Intensity, DISA Information Series, No. 17, 1978.

Flow Field Dependence on Hot-wire Probe Cooling Law and Probe Adjustment, DISA Information Series, No. 23, 1978.

"Some Remarks on Methods of Evaluating Hot-wire Output, using Squared Signals, DISA Information Series, No. 24, May 1979.

Mesures des Tensions de Reynolds dans un Écoulement Dissymétrique en Régime

Turbulent Incompressible, Journal de Mechanique, Vol. 4 No. 3, Septembre 1965

AG ARDograph No. 1991973.

An Introduction to Turbulence and its Measurement. : Pergamon Press, Oxford, $197 i$, pp. 106-132.

Experimental Fluid Mechanics, Pergamon Press, . 1970.

The Analogy between Streamline Curvature and Buoyancy in Turbulent Shear Flows, Journal of Fliud Mechanics.

Vol. 36, Part 1, pp. 177-191, 1969

Turbulent Wall Jets with and without an External Stream, Aeronautical Research Conncil, R\&M 3252, 1960.

Methods for the Measurement of Flow in Pipes, Part 1, Orifice-Plates, Nozzles, and Venturi Tubes, British Standard Institution, 1964. 

21. ESKINAZI, S. YEH, $\mathrm{H}$.

22. ESKINAZI, S. ERIAN, F.F.

23. FEKETE,G.I.

24. FERNHOLZ, H.H. VAGT, J.D.

25. GARTSHORE, I.S. NEWMAN, B.G.

26. GILES, J.A

HAYS, A.P SAWYER, R.A

27. GOODEN, R.K

28. GUITTON, D.E

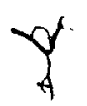

29. GUITTON, D.E. NEWMAN, B.G.
An Investigation on Fully Developed Turbulent

Flows in a Curved Channel, Journal of the

Aeronautical Sciences, Vol. 23, January 1956.

Energy Reversal in Turbulent Flows, The Physics of Fluids, Vol. 12, No. 10, October 1969.

Coanda Flow of a Two-Dimensional Wall Jet on the Outside of a Circular Cylinder, Mech. Engineering Research Laböratory Report No. 63-11, McGill University, Mòntreal, August 1963.

Turbulence Measurements in an Adverse Pressure Gradient S-D Turbulent Boundary Layer along Circular Cylinder, Journal of Eluid Mechanics, Vol. 111, pp. 233-269, 1981.

The Turbulent Wall Jet, in an Arbitrany Pressure Gradient, Aerónautical Quarterly, February 1969.

Turbulent Wall Jets on Logarithmic Spiral Surfaces, The Aeronautical Quarterly, Vol. 17, August 1966

Experimental Investigation of Boundary Layer

- Control by Tangential Injection, Master's

Thesis, Carleton University, Ottawa, July 1977.

Twö-Dimensional Turbuilent Wall Jets Over Curved Surfaces, Mech. Engineering Research Laboratory Report No. 64-7, McGill University, Montreal, June 1964.

Self-Preserving Wall Jets over Convex Surfaces, Journal of Fluid Mechanics, Vol. 81, pp. 155-185, 1977 . 
30. HINZE, J.O.

31. IRWIN, H.P.A.H.

32. IRWIN, H.P.A.H.

33. JøRGENSEN, F.E.

34. KELLY, W.J.

35. KIND;, R.J.

36. KINंD, R.J. MAULL, D.J.

37. KIND, R.J. $\therefore$

38. KIND, R.J. GOODEN, K. DVORAK; F.A.
Turbulent Flow Regions with Shear Slress and : Hean Velocity Gradient of Opposite Sign, Applied Science Research, No. 22, May 1970

Applications of a Reynolds Stress Model to :- L) Incompressible Flows, Proceedings of the Fifth Canadian Conaress of Applied Mechanucs, Fredericton, May 26-30, 1975.

Measurements in a Self-Preserving Plane: "Iall Ir: in a Pasitive Pressure Gradient, Journal of Flill: Mechanics, Vol. 61, Part I, pp. 33-63, 1973.

Directional Sensitivity of Wire and Fiber. Film Probes, DISA Information Sertes, No. 11, Noviember 1970.

An Investigation of Hot-wire Anemometer Techrulu." in a Turbulent Highly Sheared Flow, Master's Thesis. C,arleton University, Ottawa, December 198 i.

A Calculation Method for Boundary Layer Control by Tangential Blowing, CASI Transactions, Vol. 4, No. 2, September 1971.

A Experimental Investigation of a Low Speed Circulation Controlled Aerofoil, The Aeronautical Quarterly, May 1968.

Calculation of the Normal Stress Distribution in a Curved Wall Jet, The Aeronautical Journal of the Royal Aeronautical Society, May 1971

Megsurement of Flows with Tangential Injection and Comparison with Prediction Methods, AIAA Journal, Vol. 17, No. 7, July 1979. 
39. KIND, R.J.

SUTHANTHIRAN, K.

40. KRUKA, V.

ESKINAZI, S.

41. KUETHE, A.M.

CHOW, C.Y.

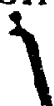

42. LAUNDER, B.E.

RODI, W.

43. LAUNDER; B.E.

RODI, W.

44. McGAHAN, W.A.

45. MOJOLA, O.D.

47.

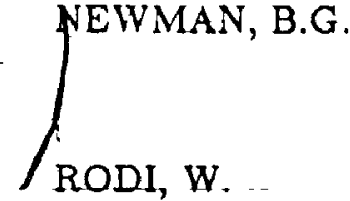

48. SIURU, W.D.

LOGAN, E.
The Interaction of Two Opposing Plane Turbulent

Wall Jets, Journal of Fluid Mechanics,

Vol. 58, Part 2, pp. 389-402, 1973.

The Wall-Jet in a Moving Stream, Journal of

Fluid Mechanics, Vol. 20, Part 4, pp. 555-579, 1964.

Foundations of Aerodynamics: Bases of

Aerodynamic Design, Third Edition,

John Wiley \& Sons Inc., 1976.

Turbulent Wall Jet Flow,

Complex Turbulent Flows, Vol. 1, The 1980-81

AFOSR-HTTM-Stanford Conference on Complex

Turbulent Flows Comparison of Computation and

Experiment.-

The Turbulent Wall Jet Flow,

Progress in Aerospace Science, Vol. 19. pp. 81-128,

\section{1}

Th Incompress ble Turbulent Wall Jet in an Adverse,

Pressure Gradient, $\mathrm{PhD}$ Thesis, MIT, 1965.

A Hot-wire Method for 9-D Shear Flows, .

DISA Information Series, No. 16, July 1974.

The Prediction of Turbulent Jets and Wall Jets, CASI Journal, Vol. 15, No. 8, pp. 288-305.

A New-Methad of Analysis of Hat-wire Signals in Highly Turbulent Flow and Its Evaluation in a Round Jet, DISA Information Series, No. 17, February 1975.

Use of Slanting Hot-wire to make Measurements in an Artificially Roughened Tube, DISA

Information Series, No. 21, April 1977. 
49. SMITH, A.M.D.

50. SUTHANTHIRAN, K.

51. TAILLAND, A. MATHIEU, J.

52. WAGNER, R.D FISCHER, M.C

53. WILSON, D.J. GOLDSTEIN, R.J.

54. WYGNANSKI, I NEWMAN, B.G.

55. YOWAKIM, F.M.

56. THERMO-SYSTEMS, INCे.

57. TOWNSEND, A.A
High-Lift Aerodtinamics, Journal of Aircralt, Vol. 12, No. 6, June 1975.

The Interaction of Two Opposing Plane Turbulent Wall Jets, Master's Thesis, Carleton Lniversity, Ottawa, March 1971.

Jet Parietal, Journal de Mecanique, 6, 103. 1!):

Fresh Attack on Laminar Flow, Aerospace America, March 1984.

Turbulent Wall Jets with Cylindrical Streamurie Surface Curvature, Journal of Fluids Engfneering, Vol. 98, Sept. 1976.

The Effect of Jet Entrainment on Lift and Moment for a Thin Aerofoil With Blowing, The Aeronautical Quarterly, May 1964.

Experimental Investigation of Turbulent Swirling Flow in an Annulus, PhD Thesis, Carleton Ùniversity, Ottawa, April 1985 Operating Manuat, 1054 A Hot-Wire Anemometer Minnesota, 1968.

The Structure of Turbulent Shear Flow. Cambridge University Press, 1956.

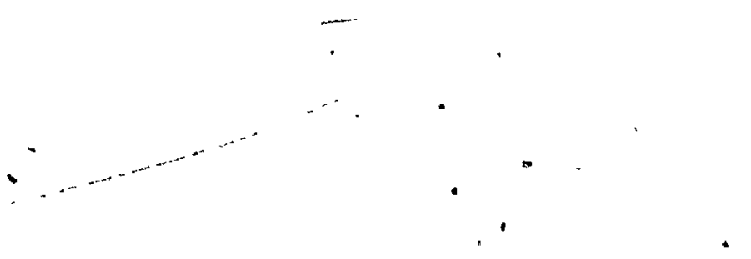




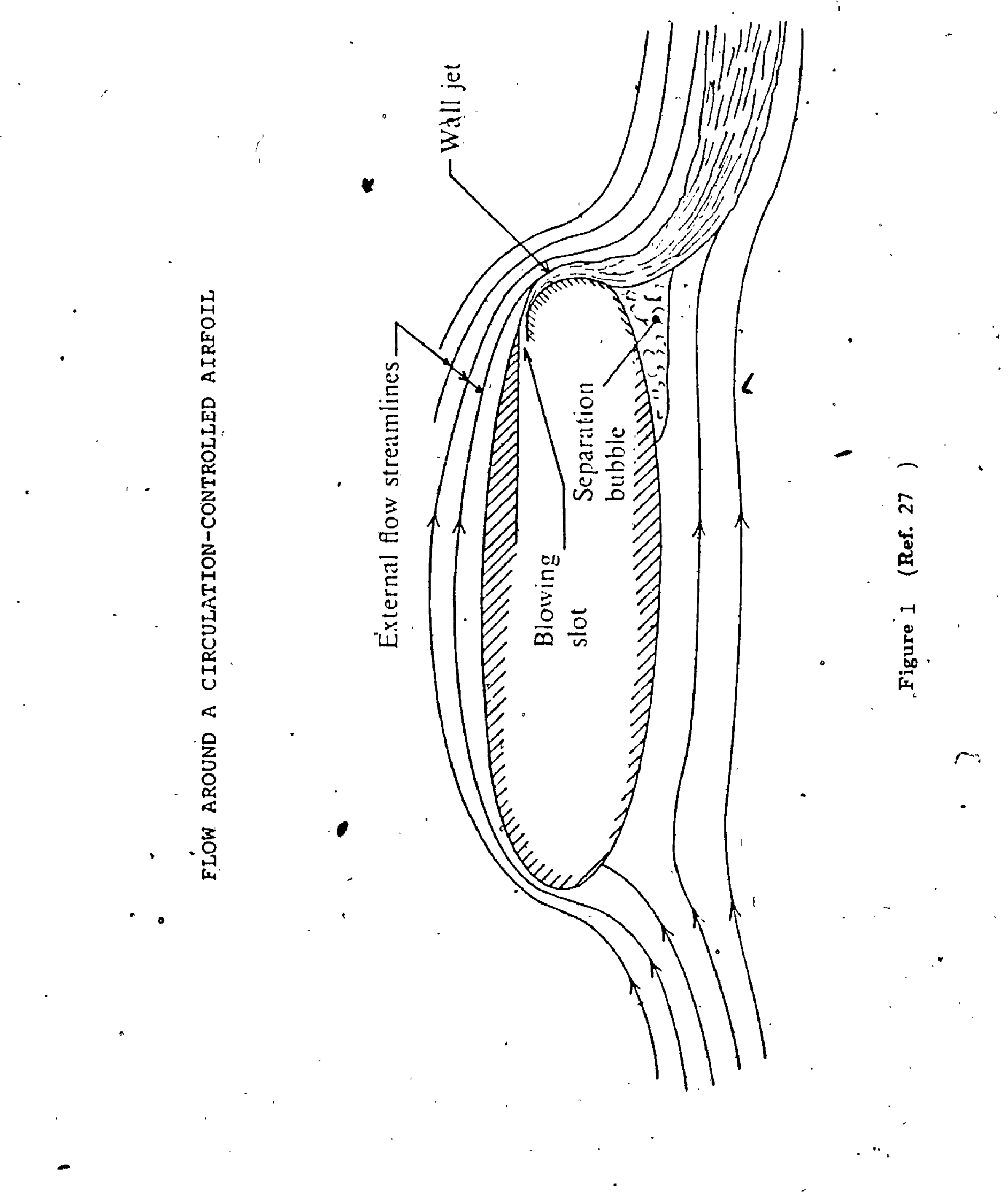


:

1

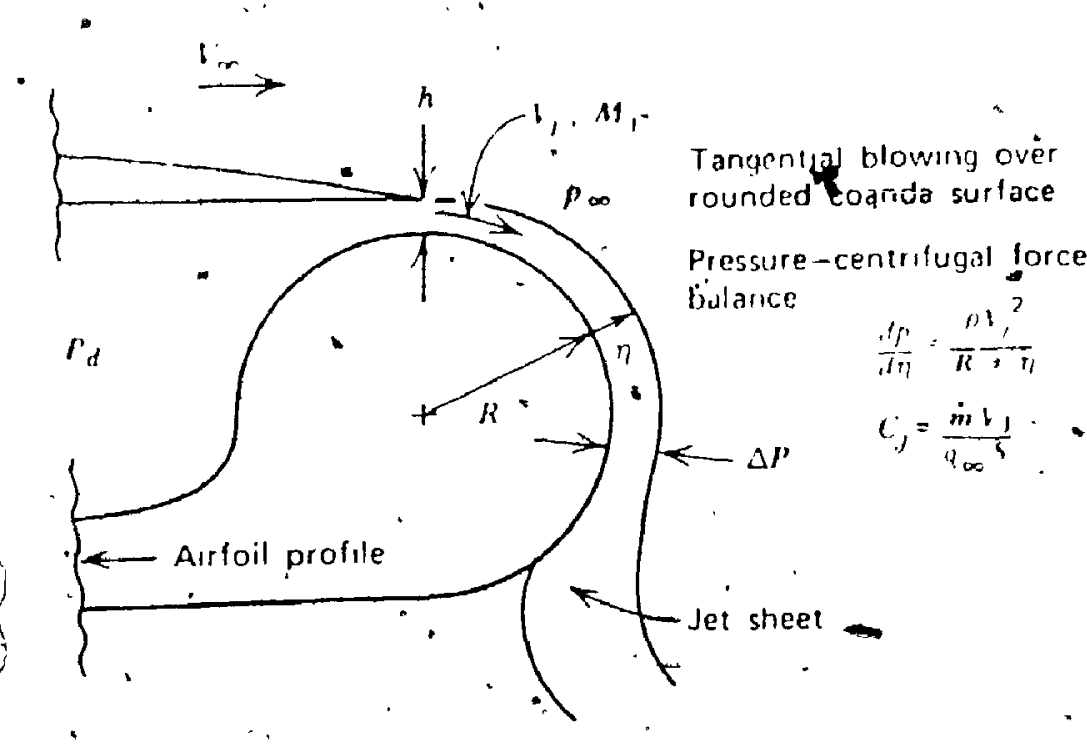

Figure 2 : Tangential Blowing over a Rounded Trailing Edge Airfoil (Ref. 18 - )

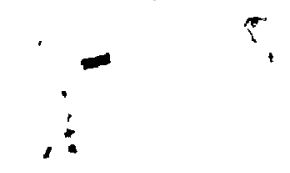



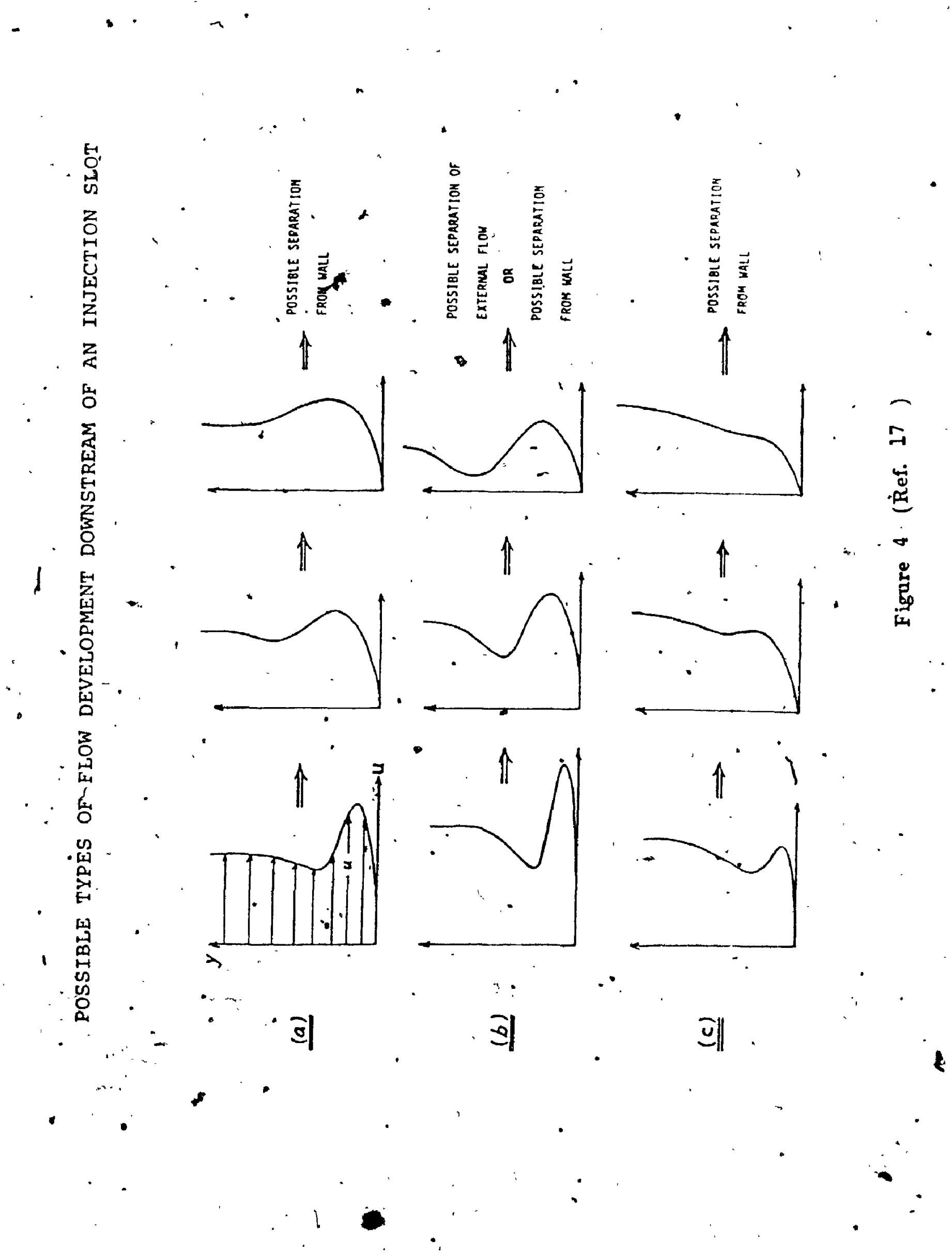
Nomenclature for Cylinder Wall-Jet Velocity Profle

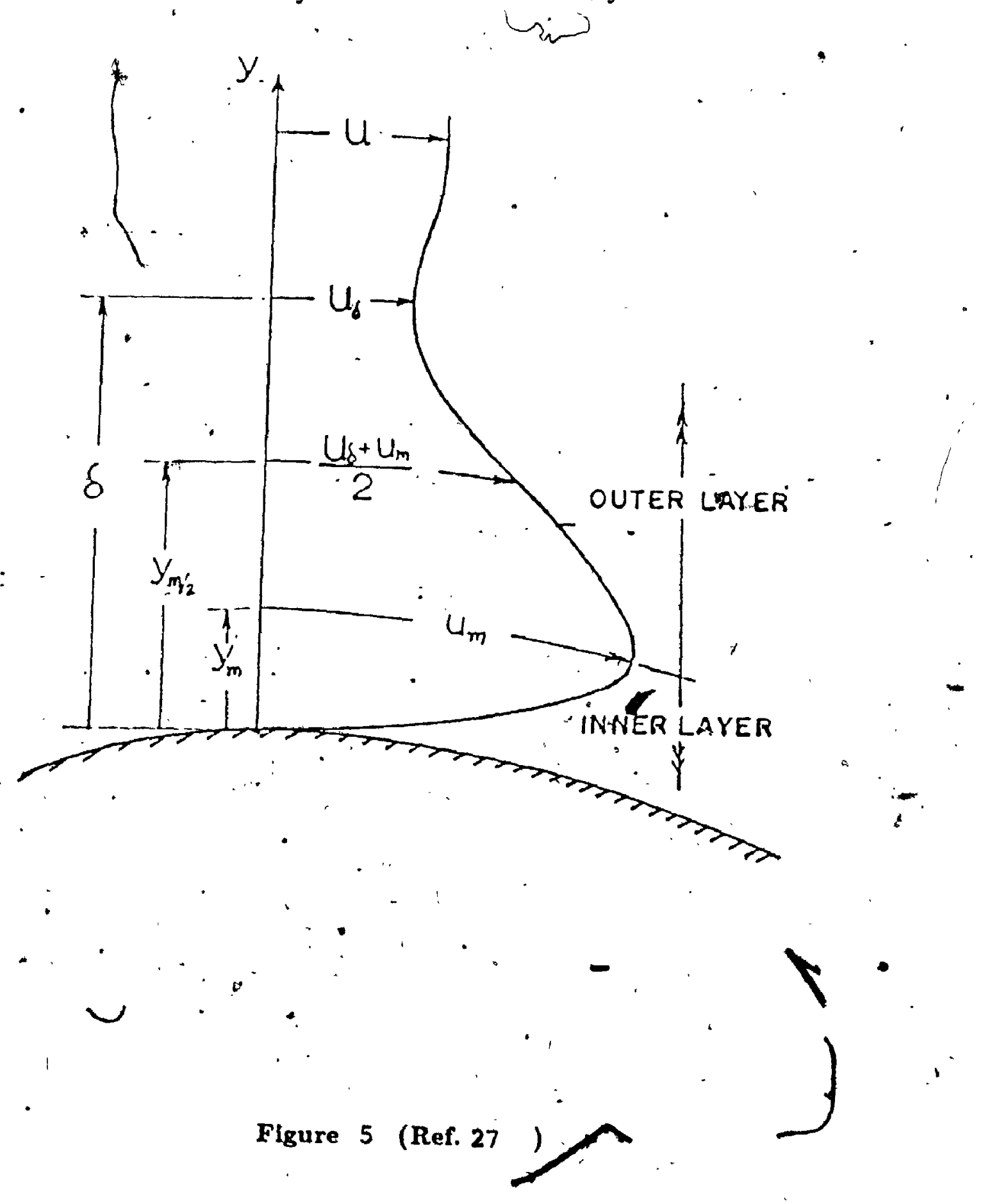




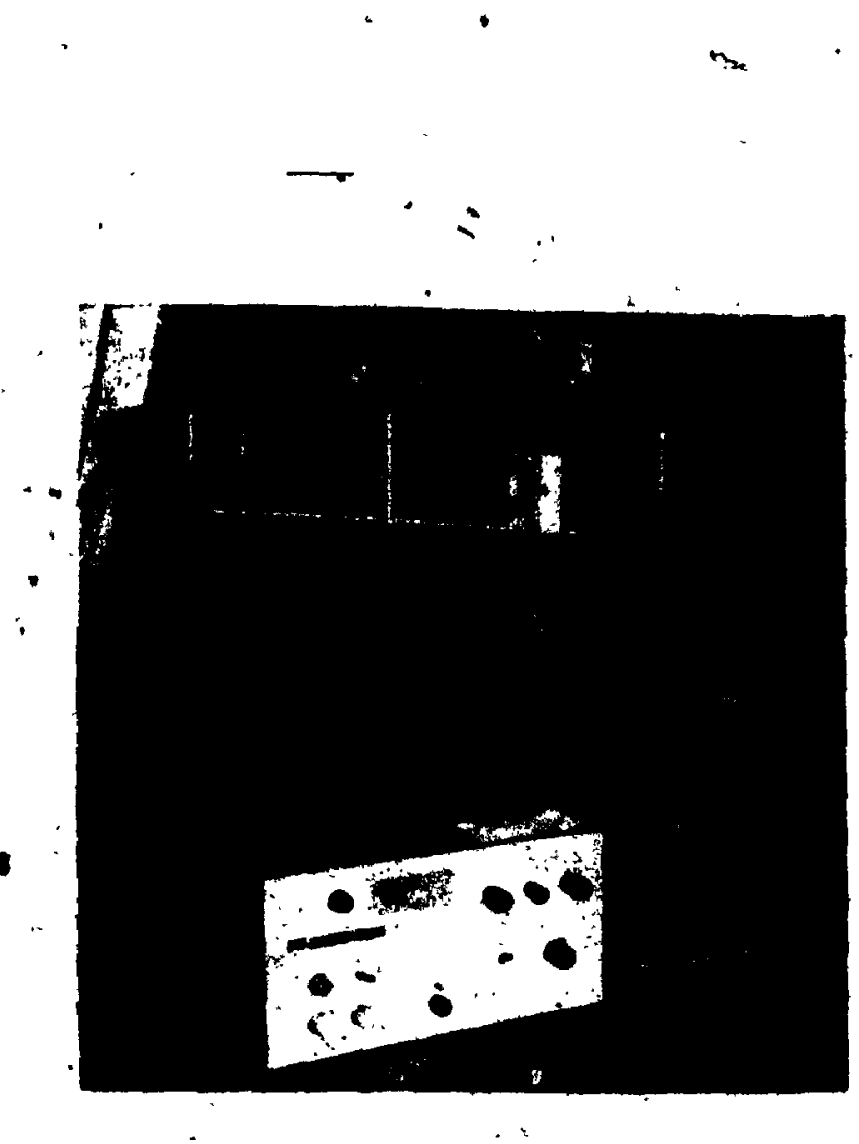

Figure " 6 : Photograph of the Plane Wall-Jet Apparatus

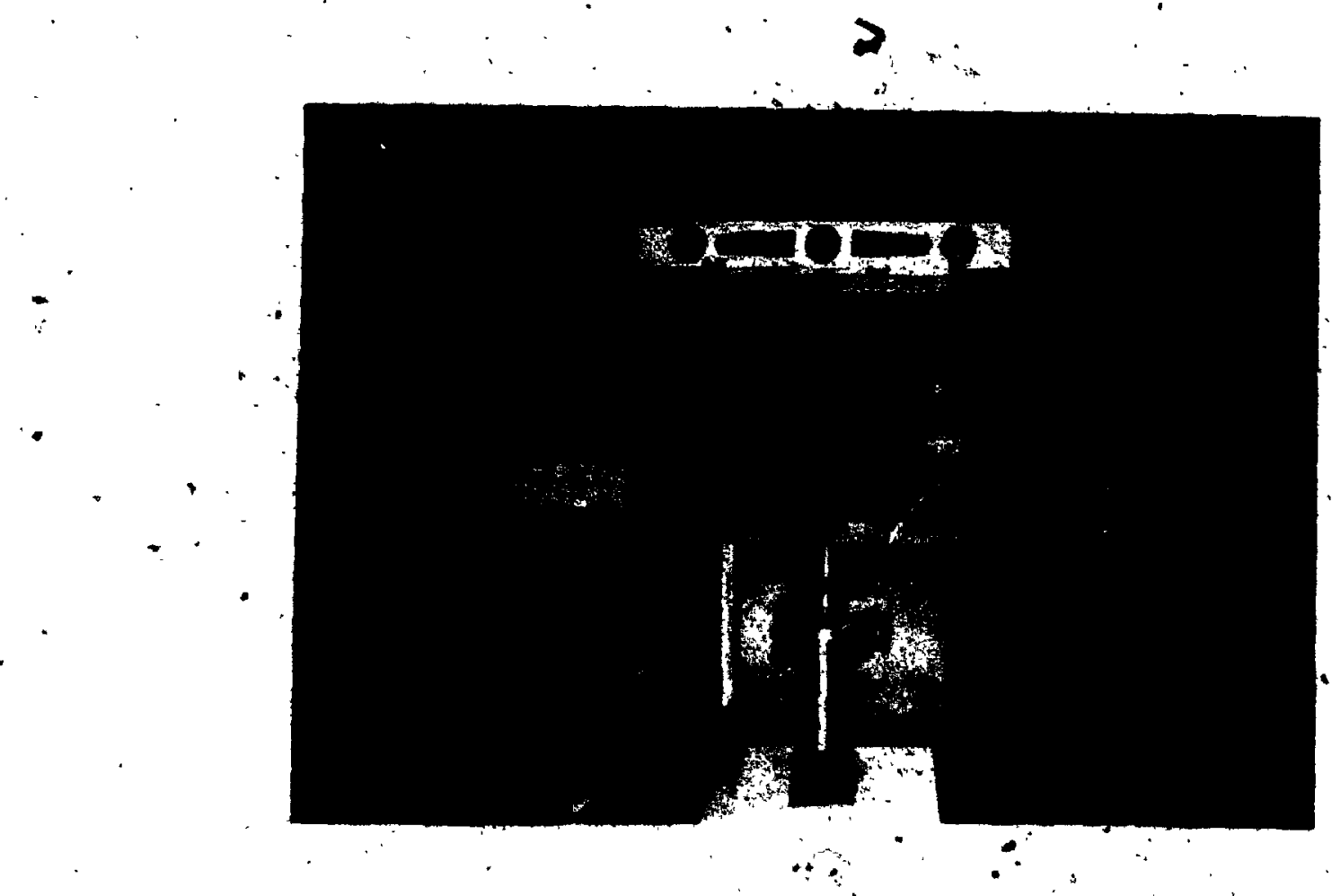

Figure 7 : Photograph of the Callbratlon Apparatus 


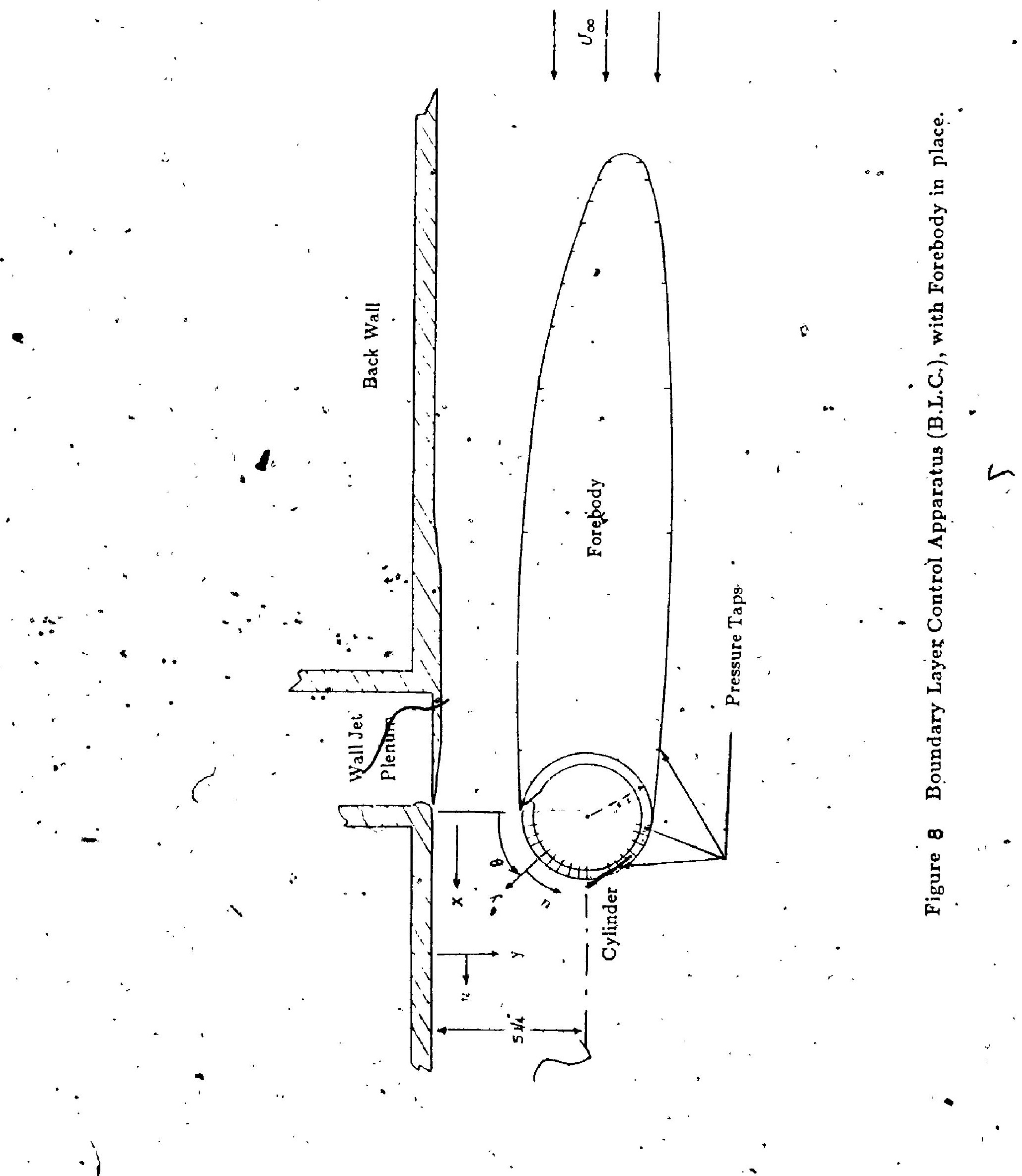




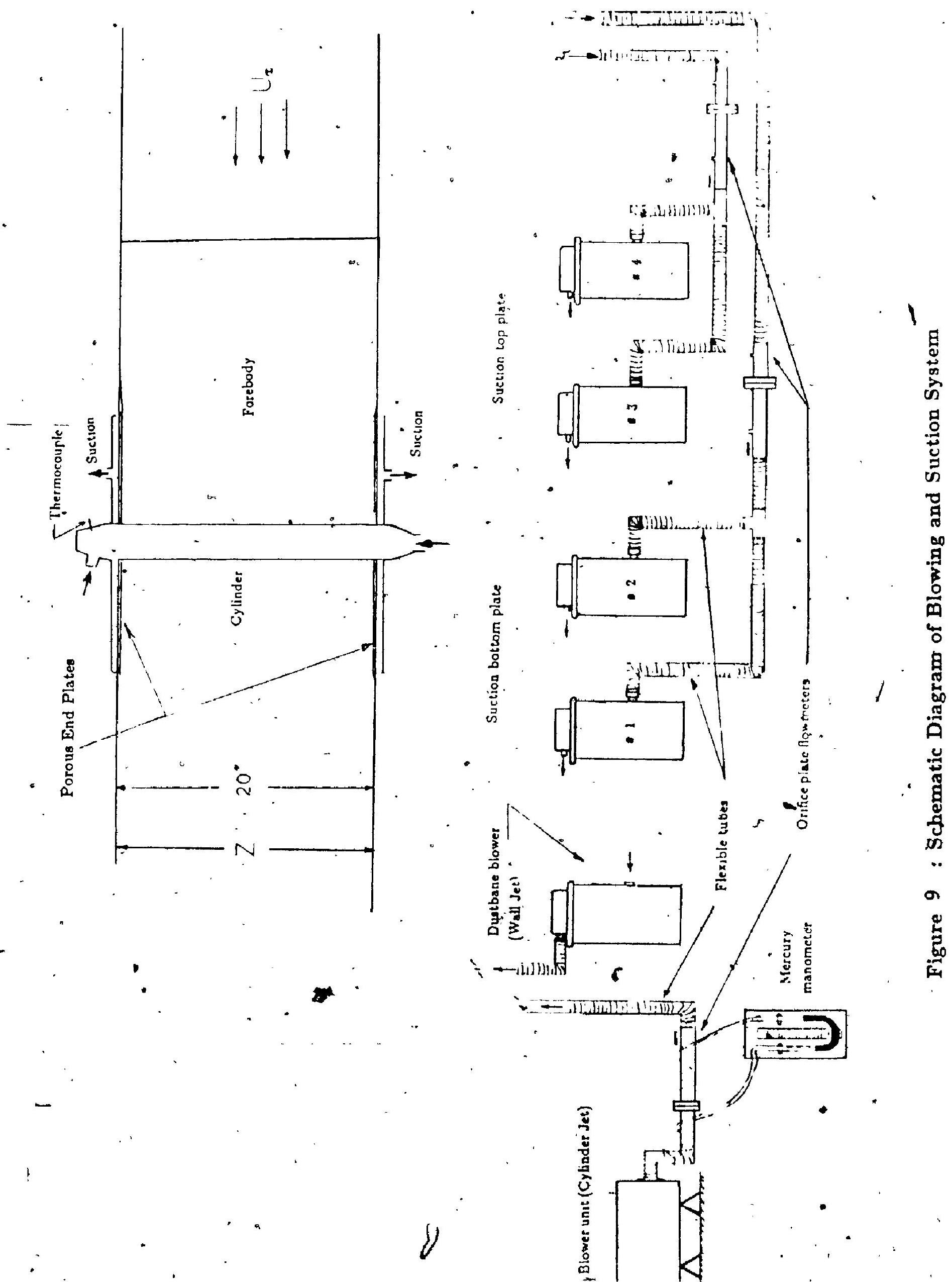




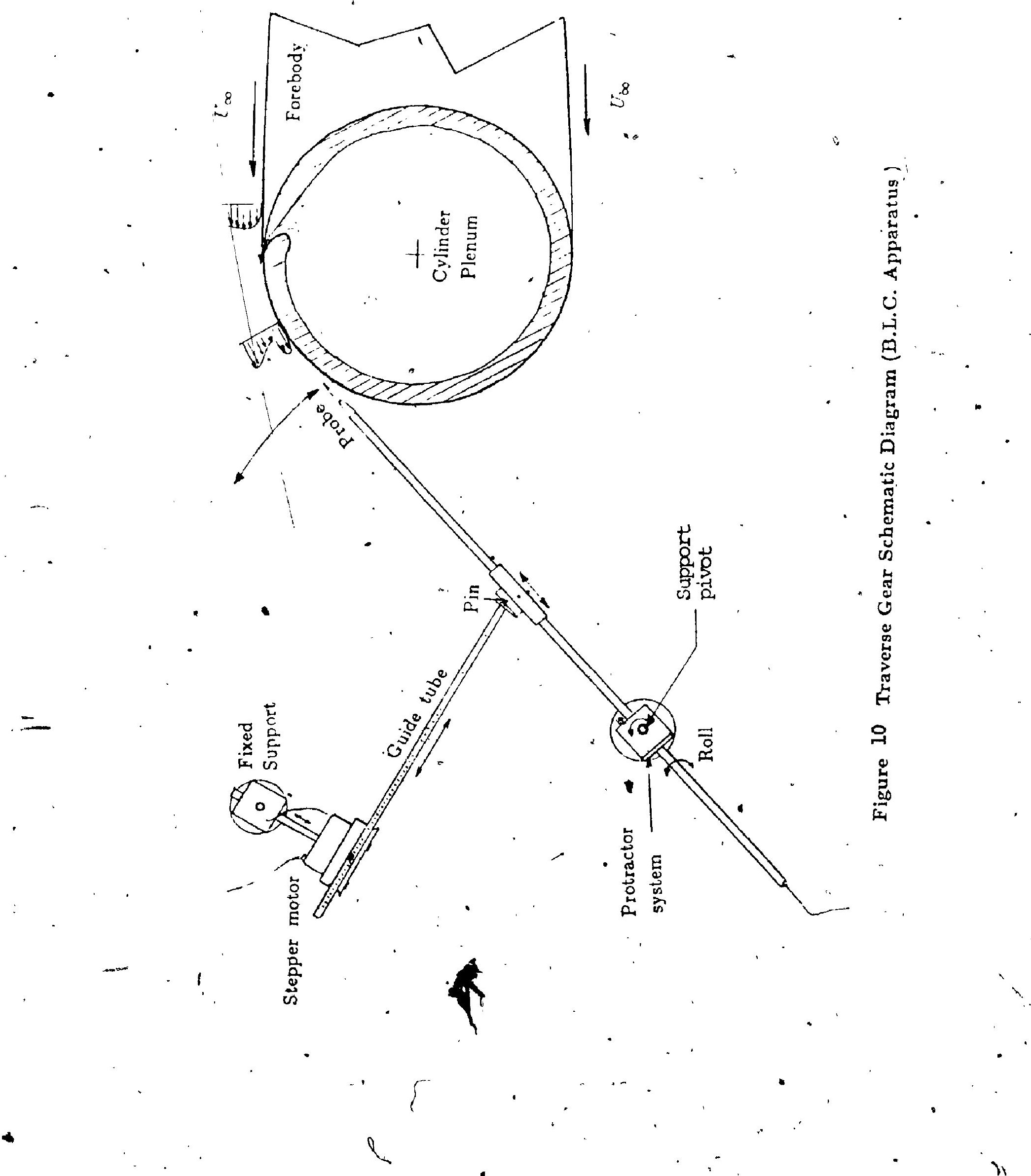




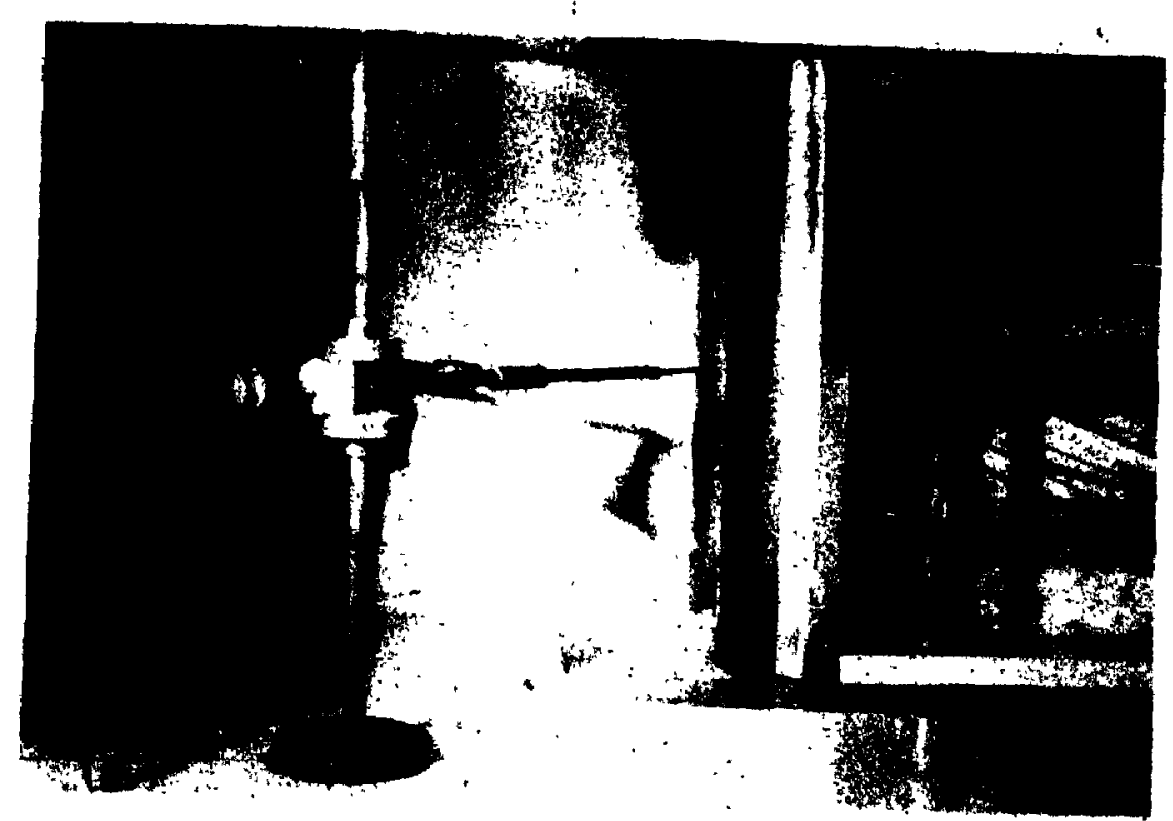

Figure 11: Photograph of the Traversing Gear in Place

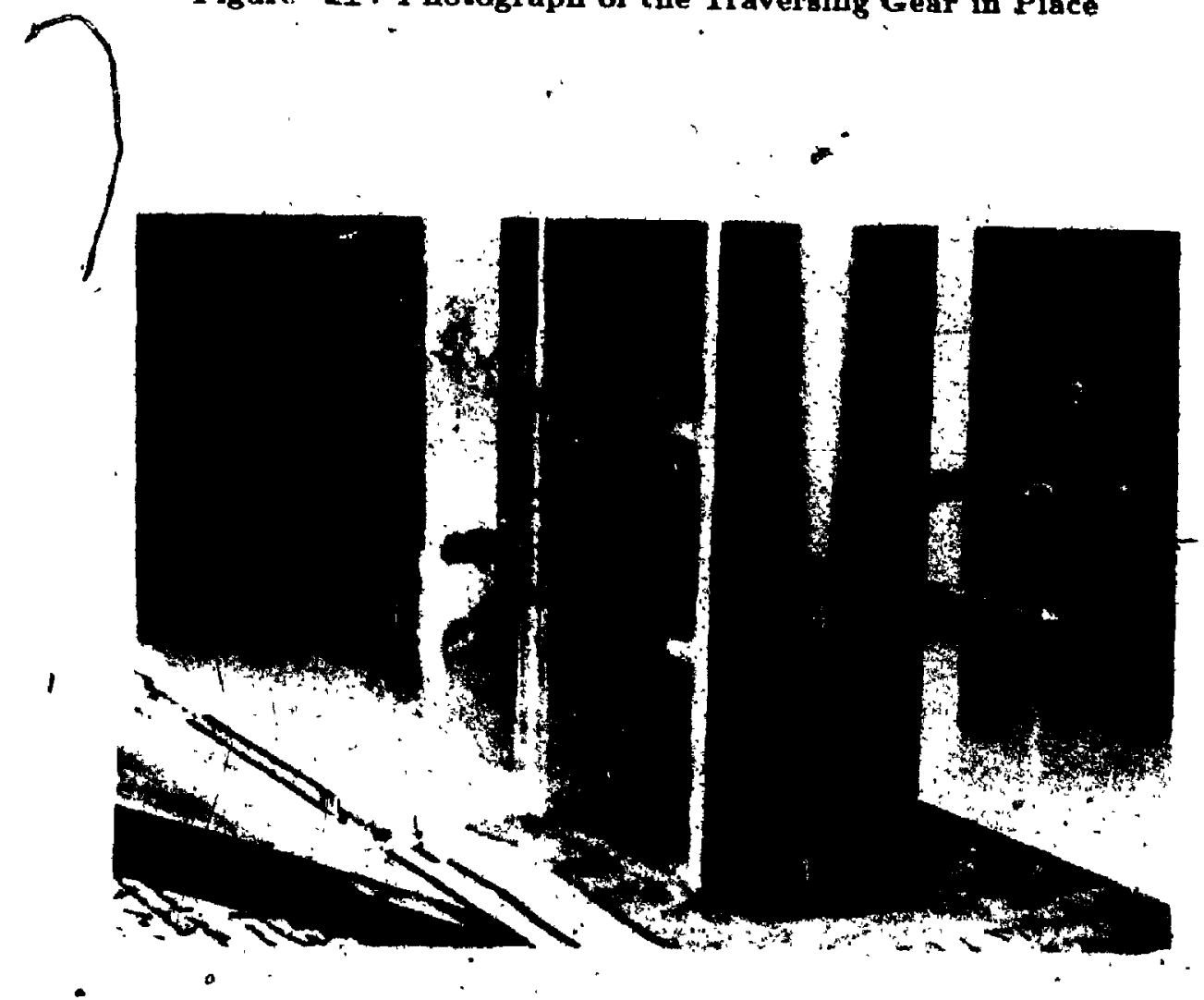

Figure 12: Photograph of the Rear-side of B.L.C.Apparatus 


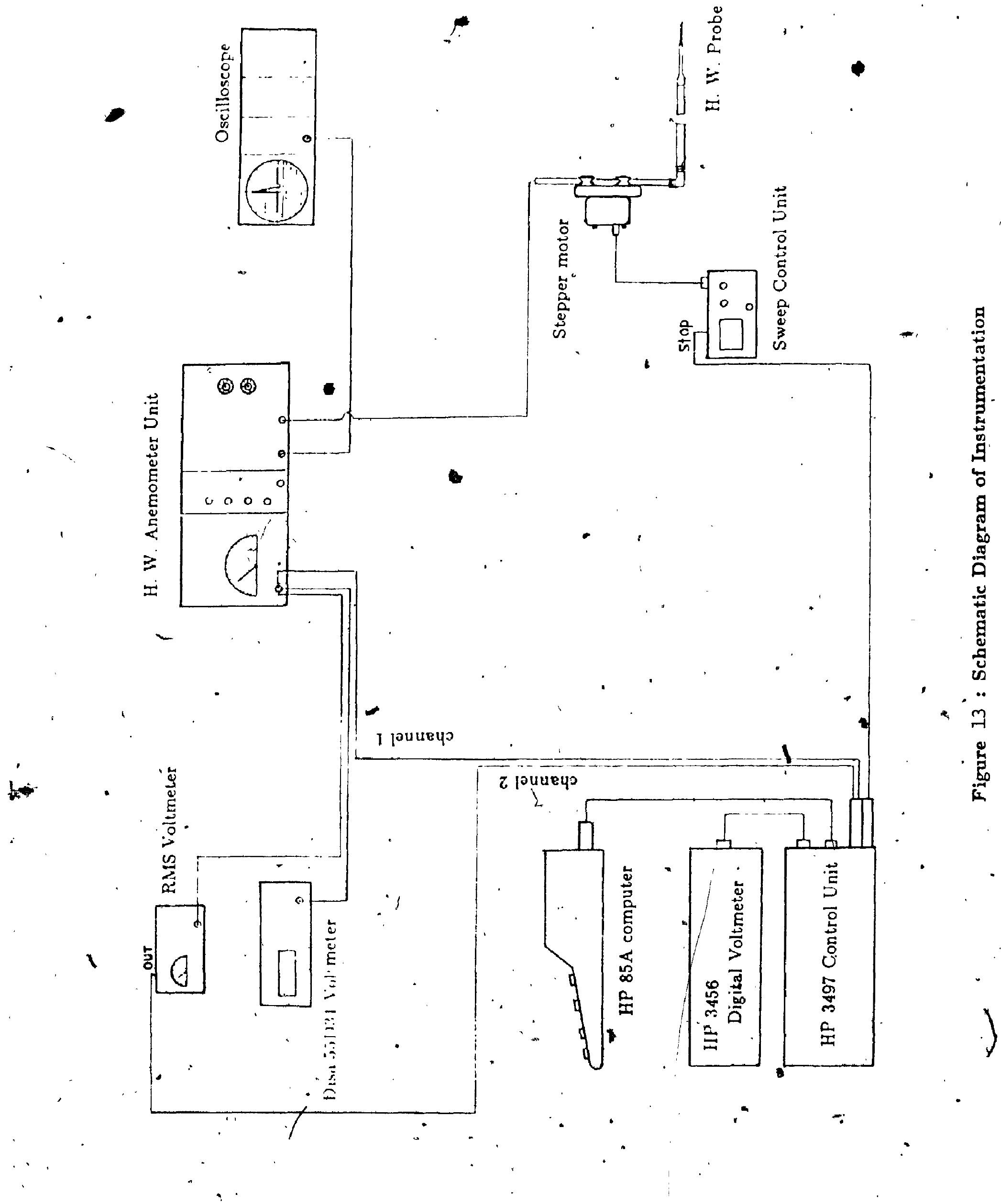



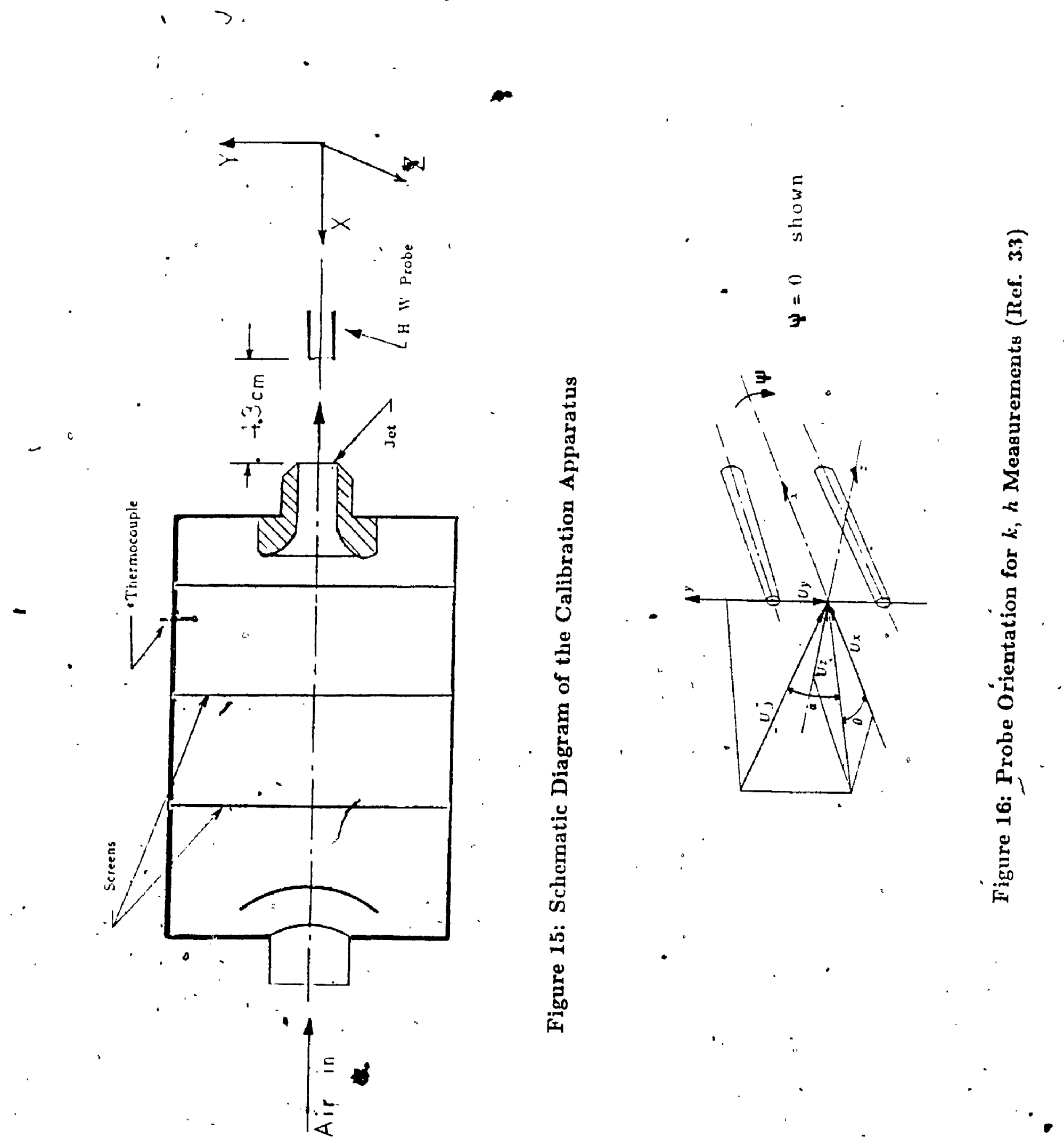


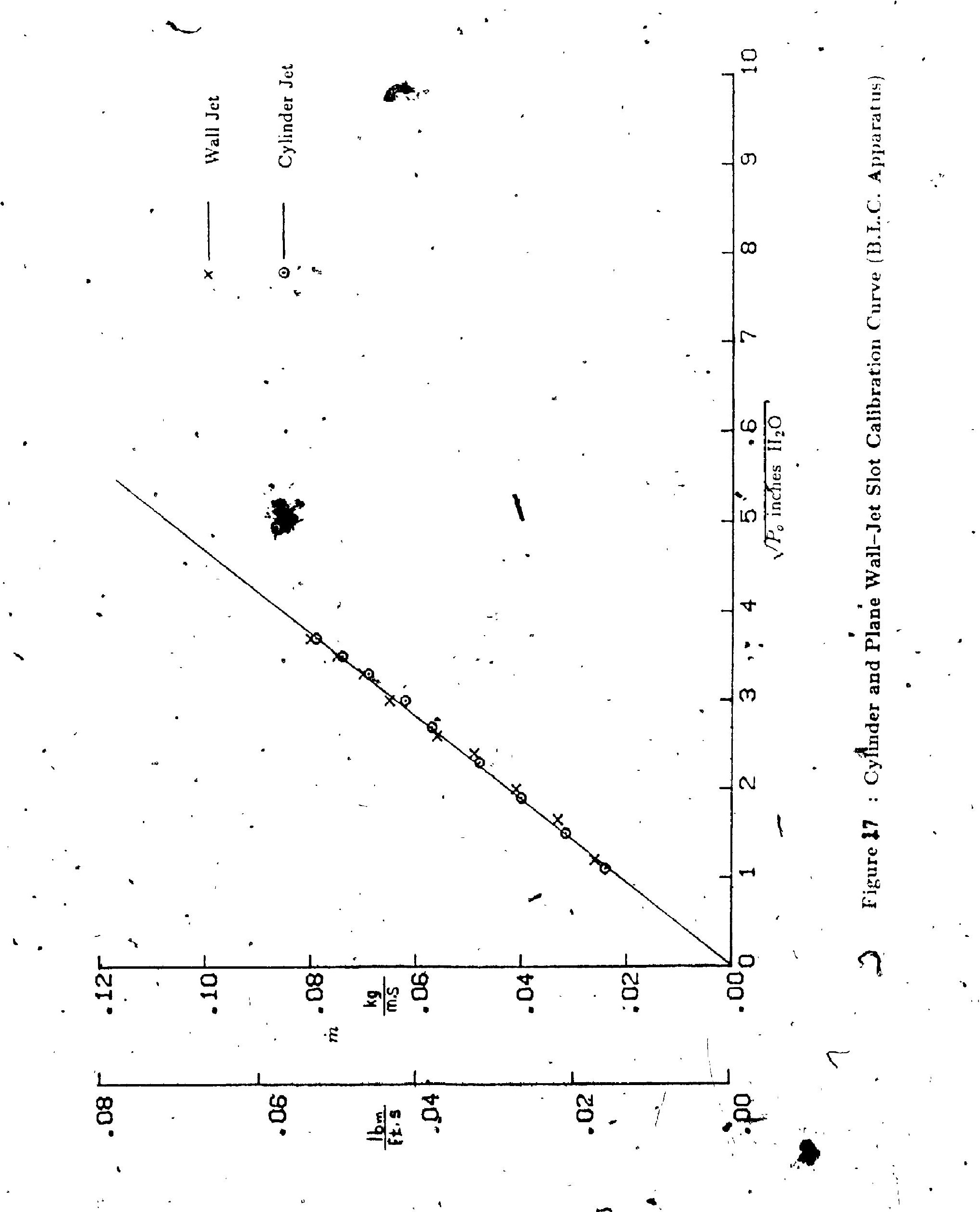


5

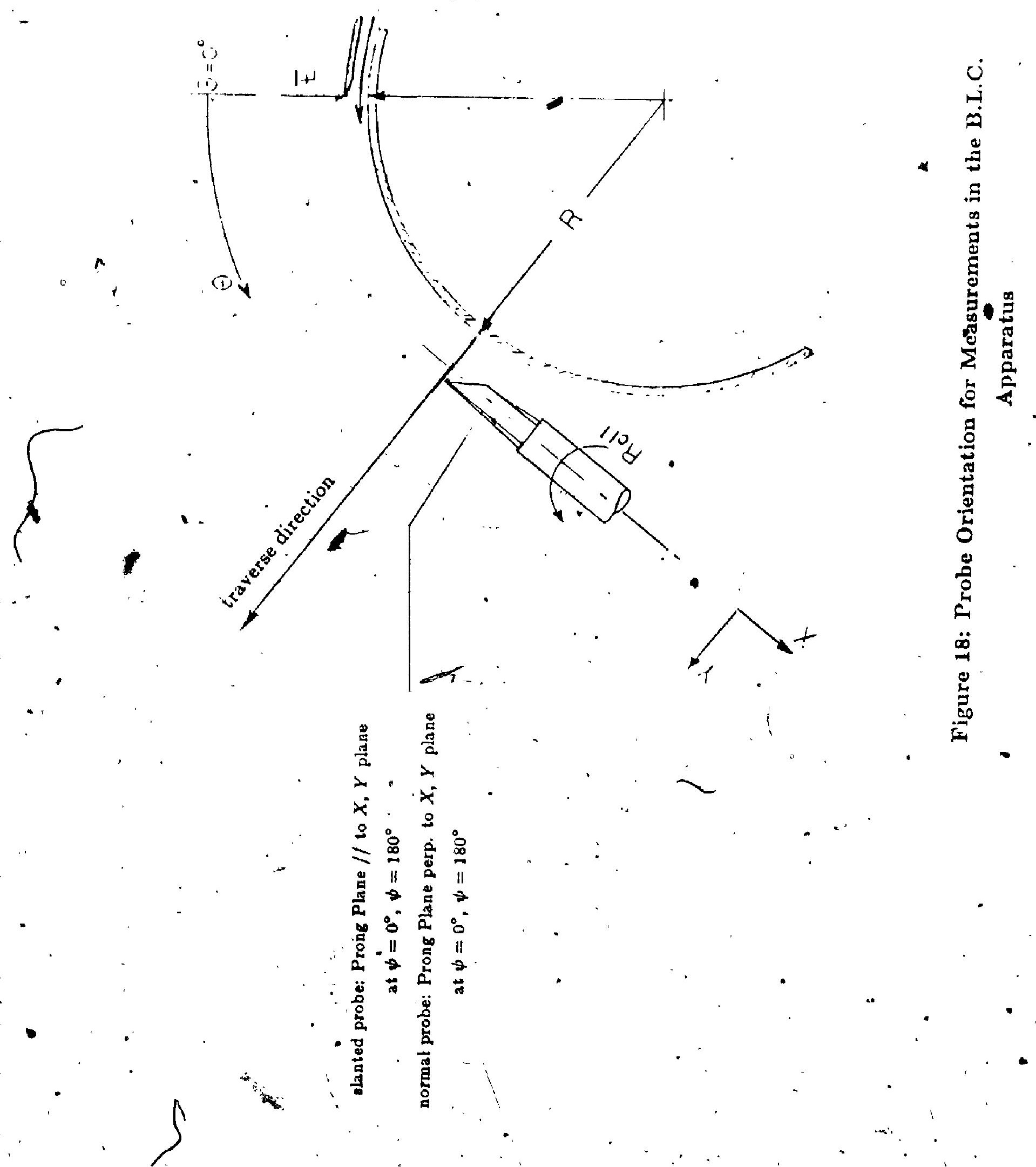




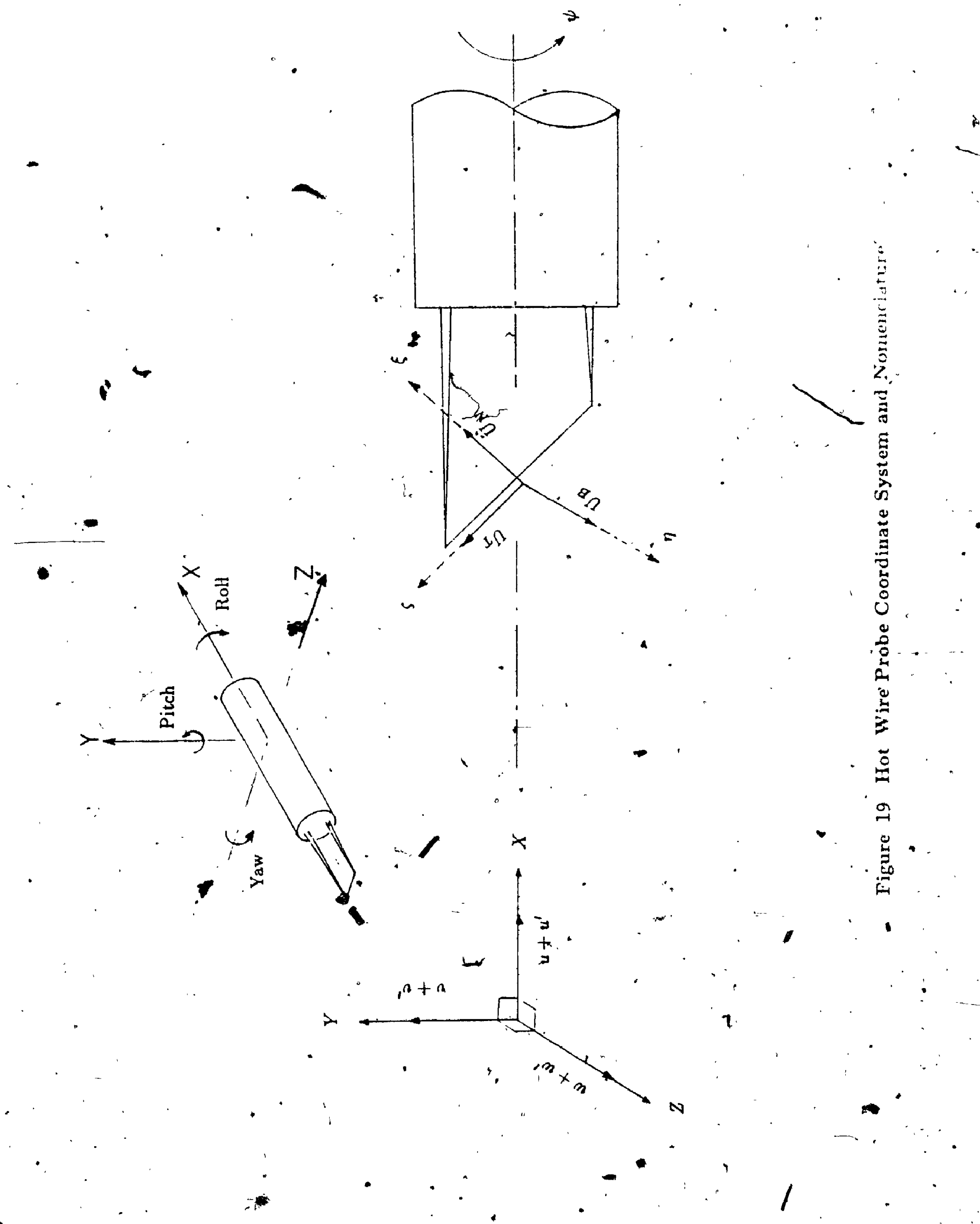



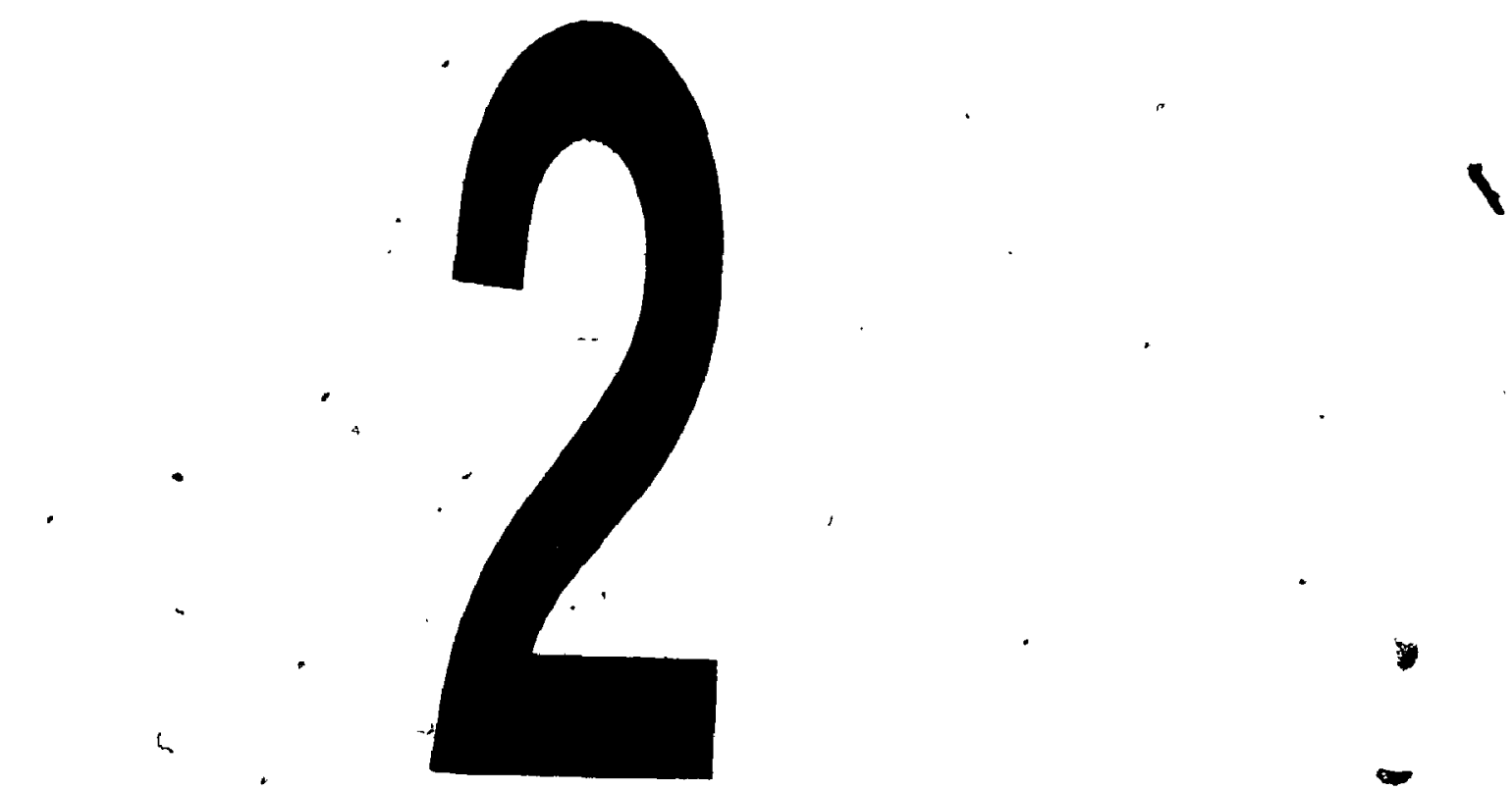

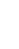
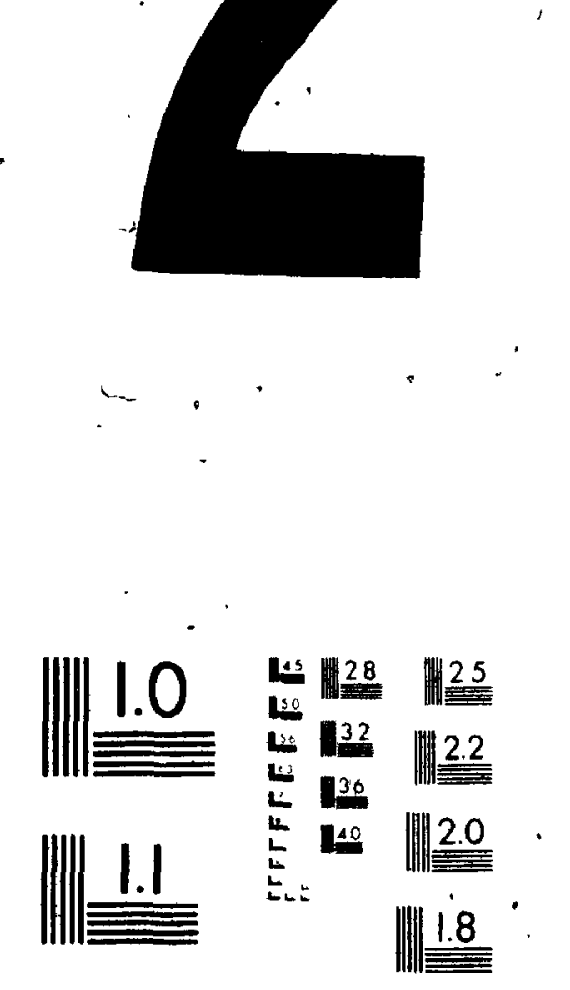

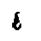

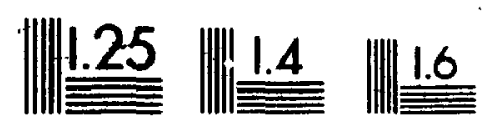

\author{
MICROCOPY RES̄OLUTION TEST CHART \\ NATIONAL BUREAU OF STANDARDS \\ STANDARD REFEAENCE MATERIAL 1010a \\ (ANSI and ISO TEST CHART No 2)
}




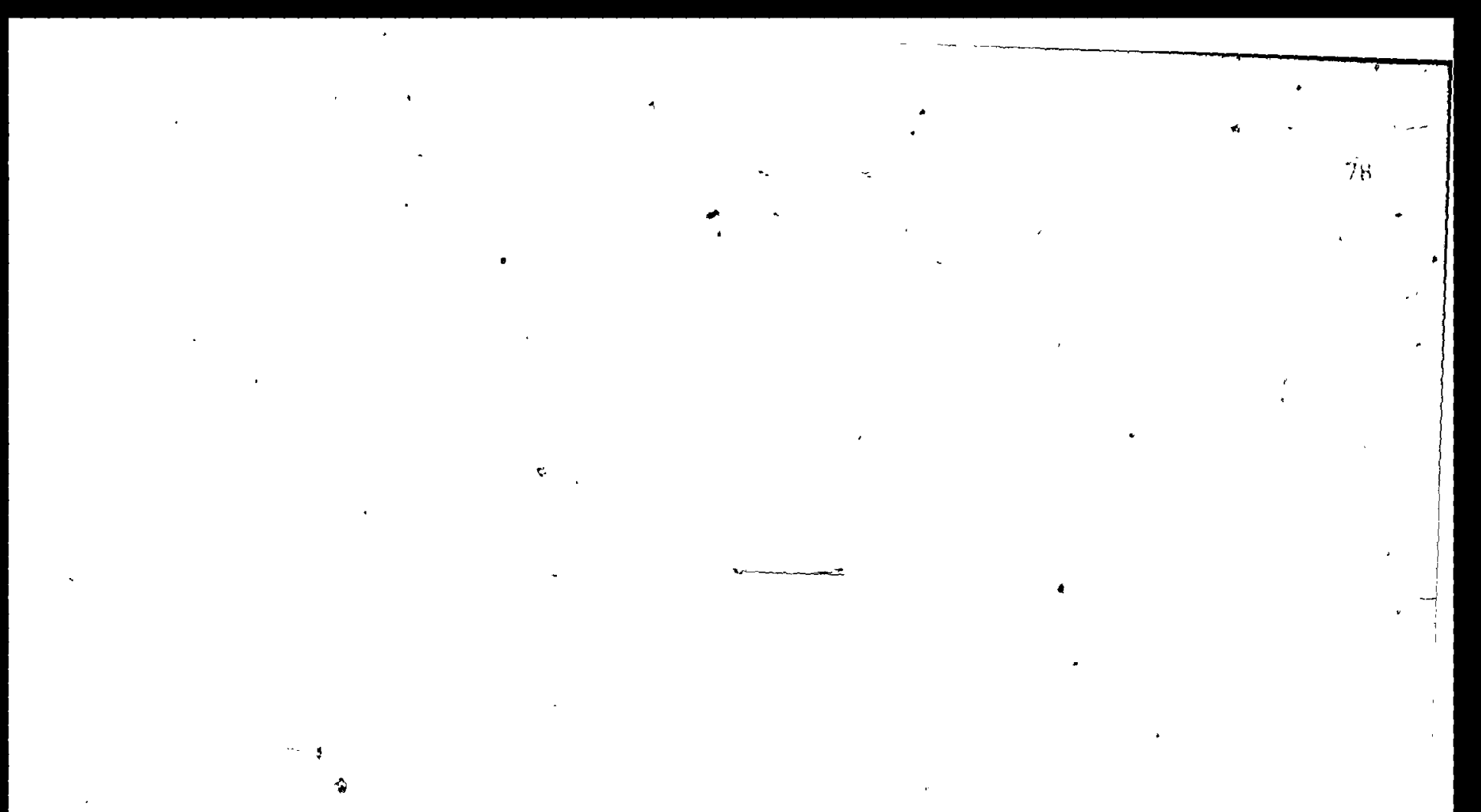

Results for the Plane Wall-Jet Apparatus . (Still Surroundings) 


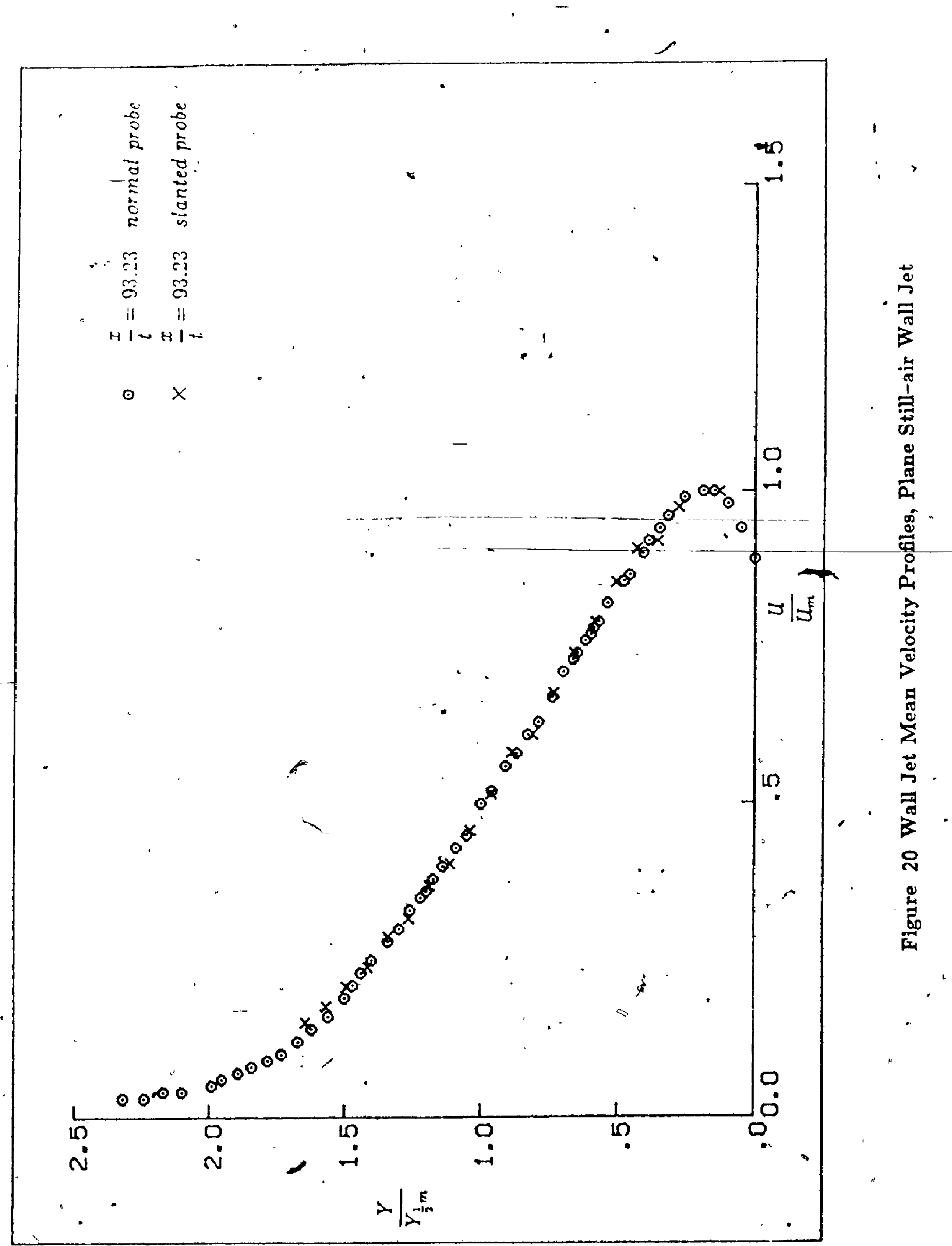




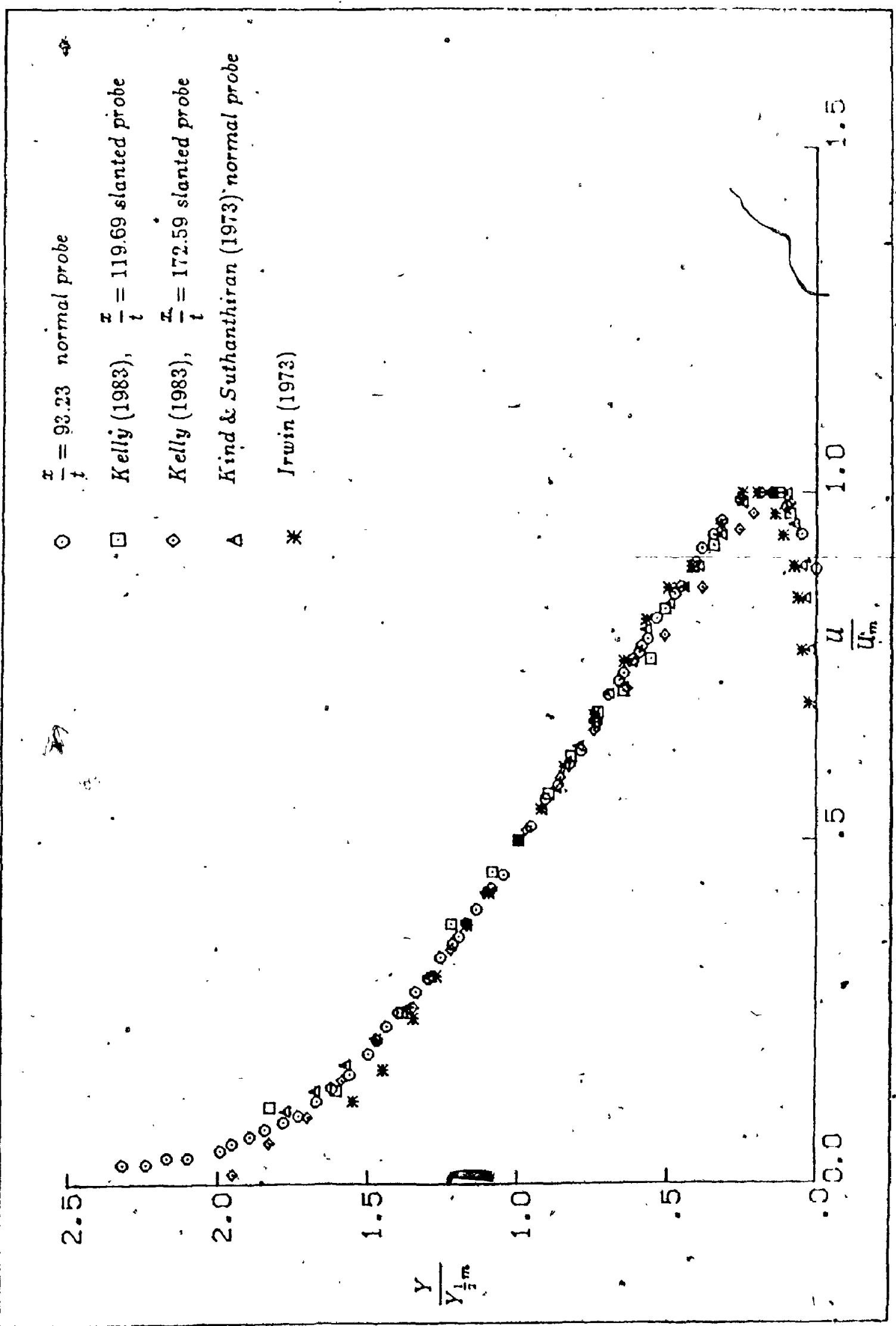




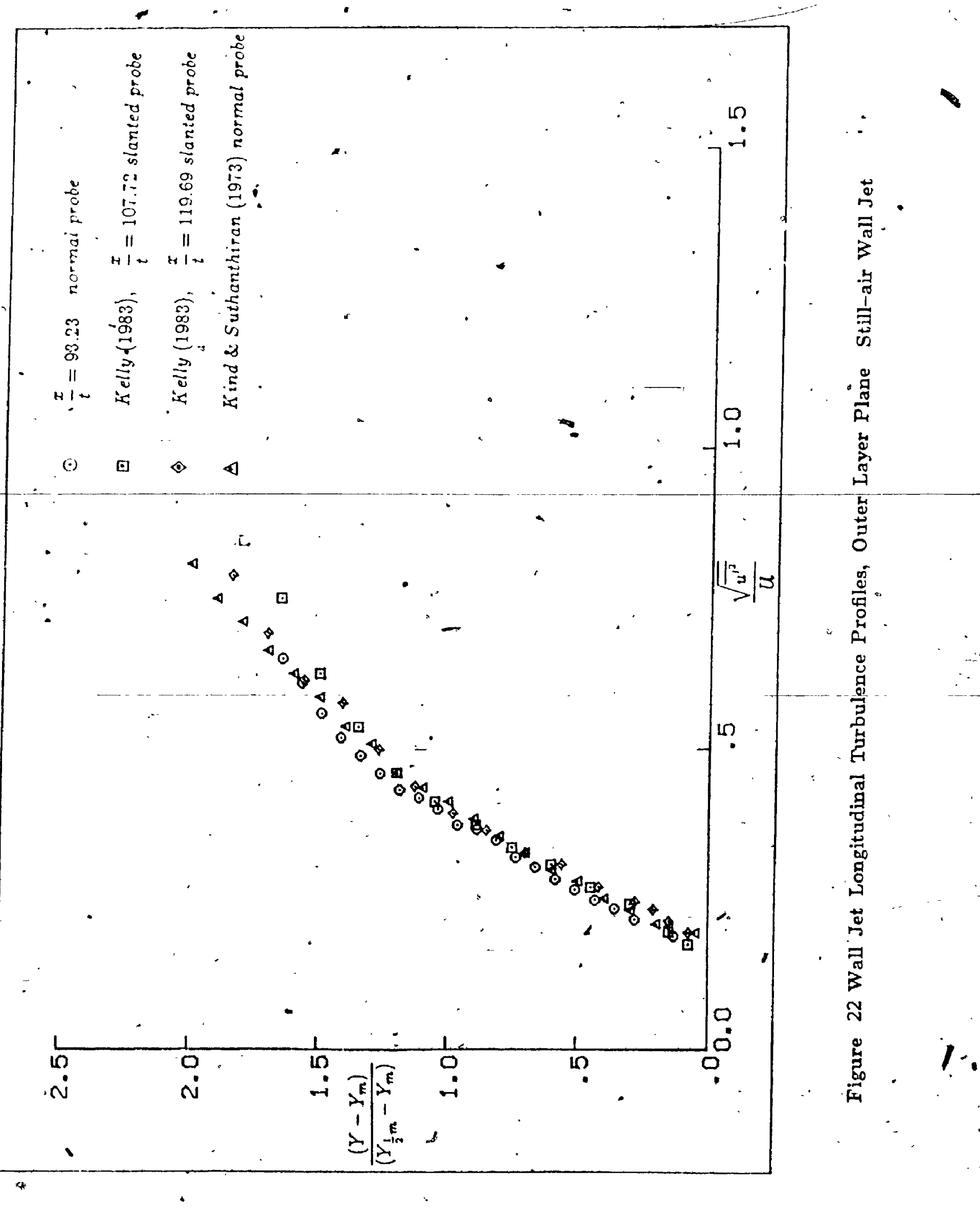




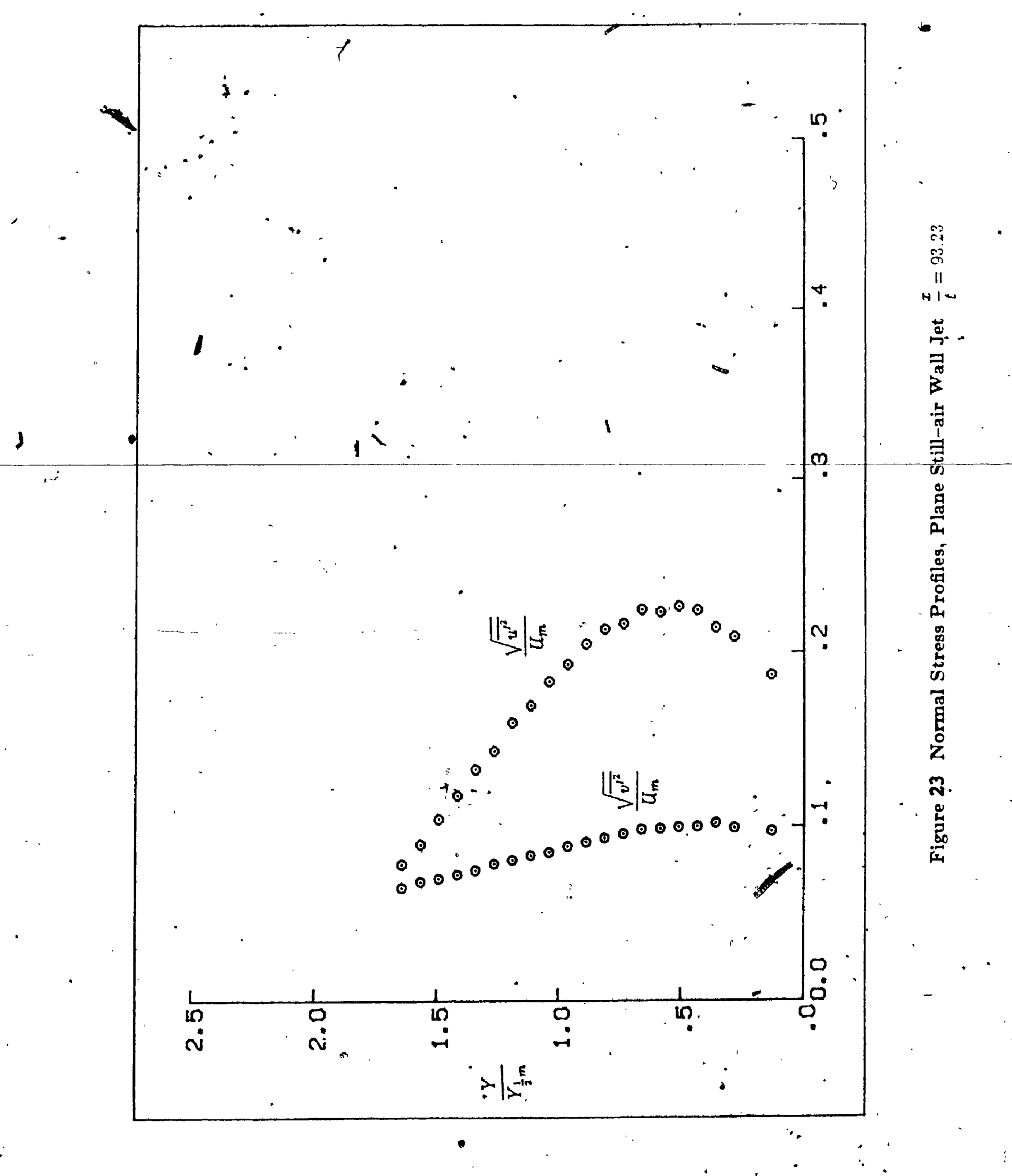




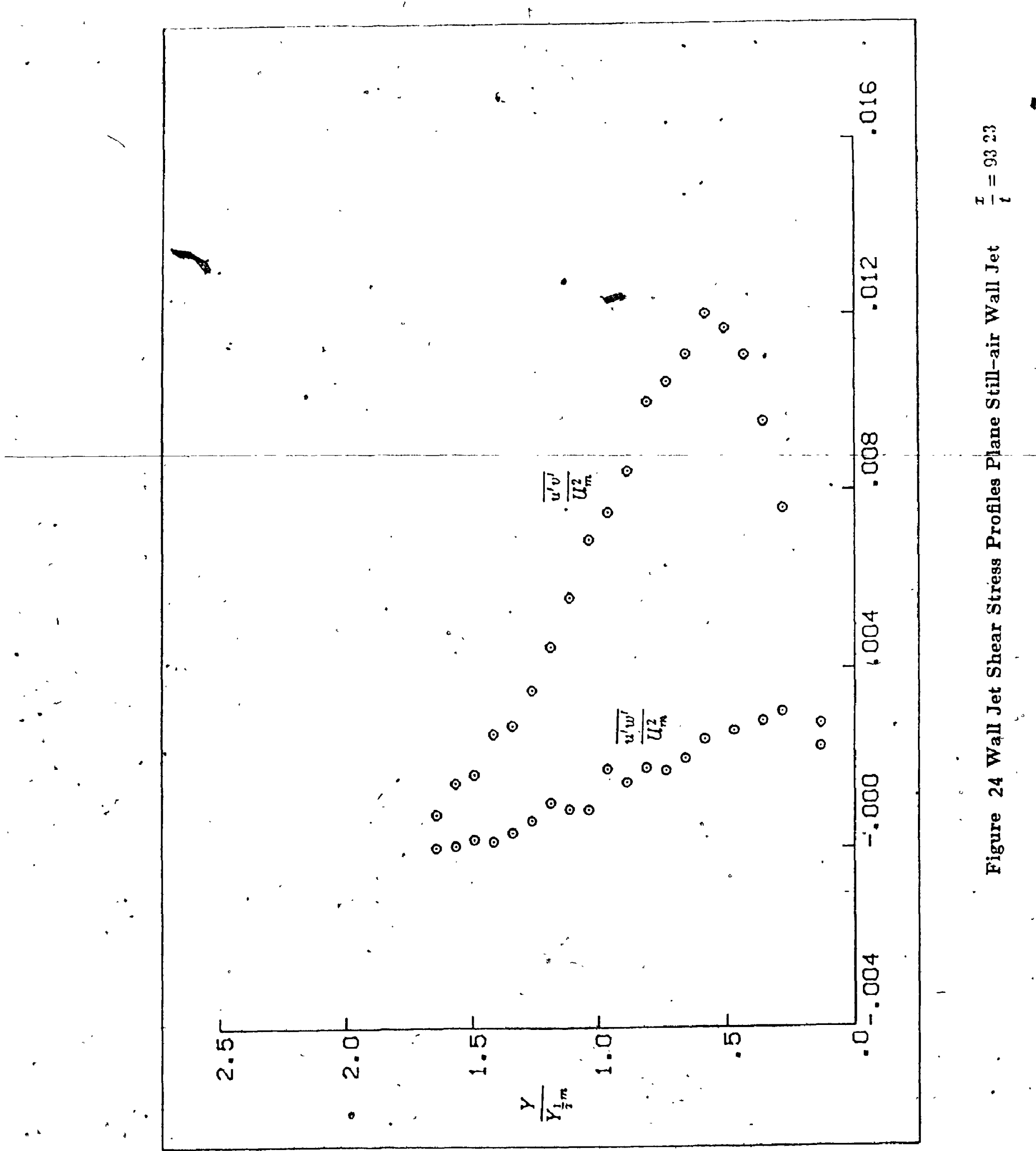




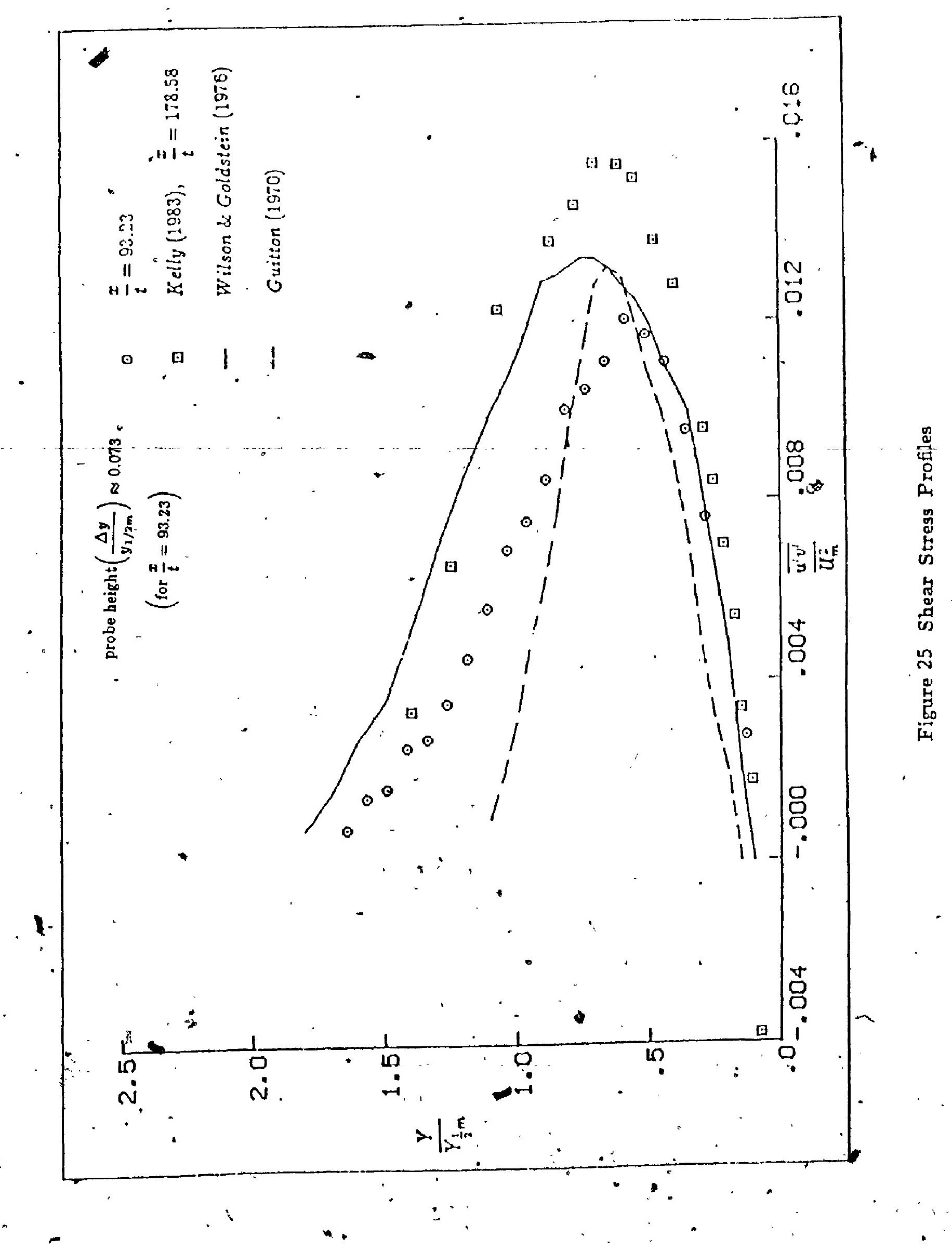





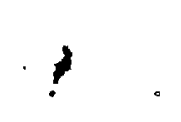

$\therefore$

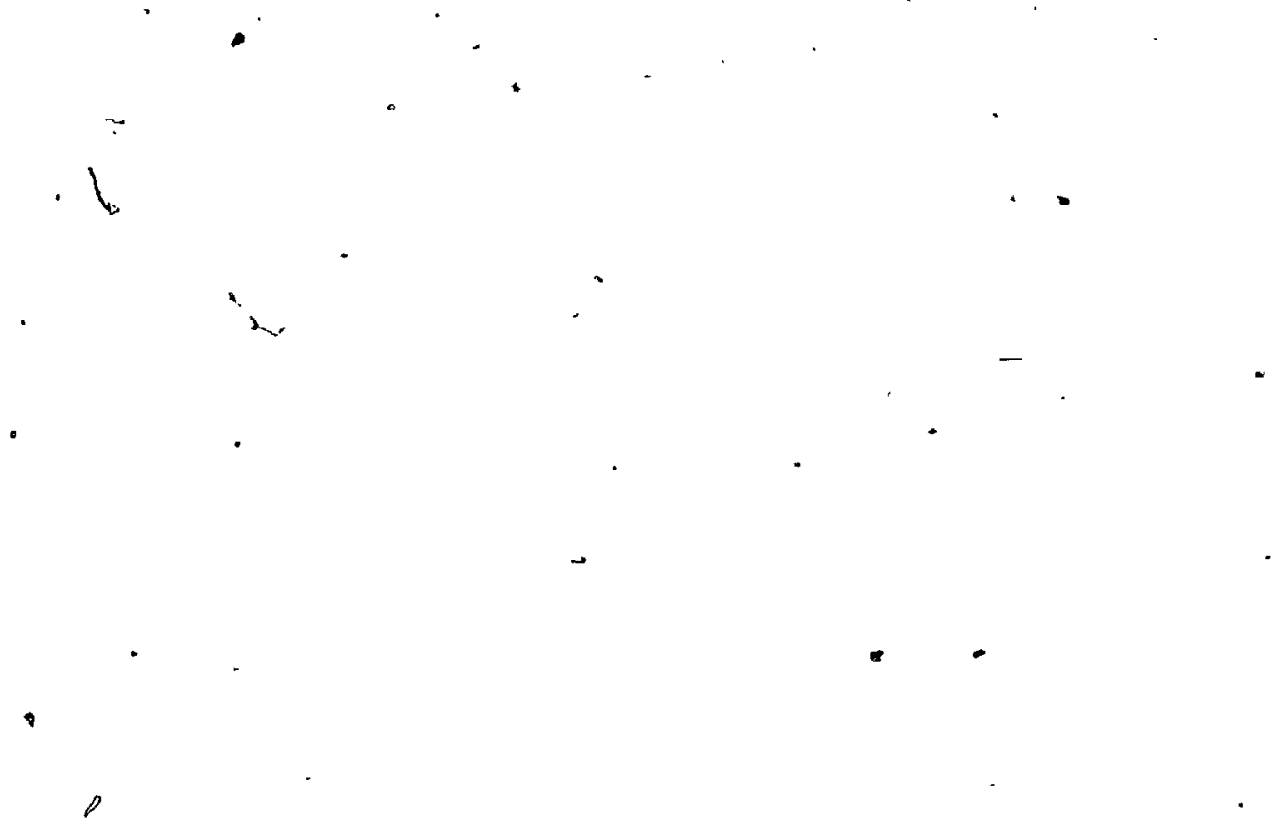

\section{Results for the B.L.C. Apparatus}

$+\ldots$

,

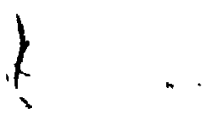




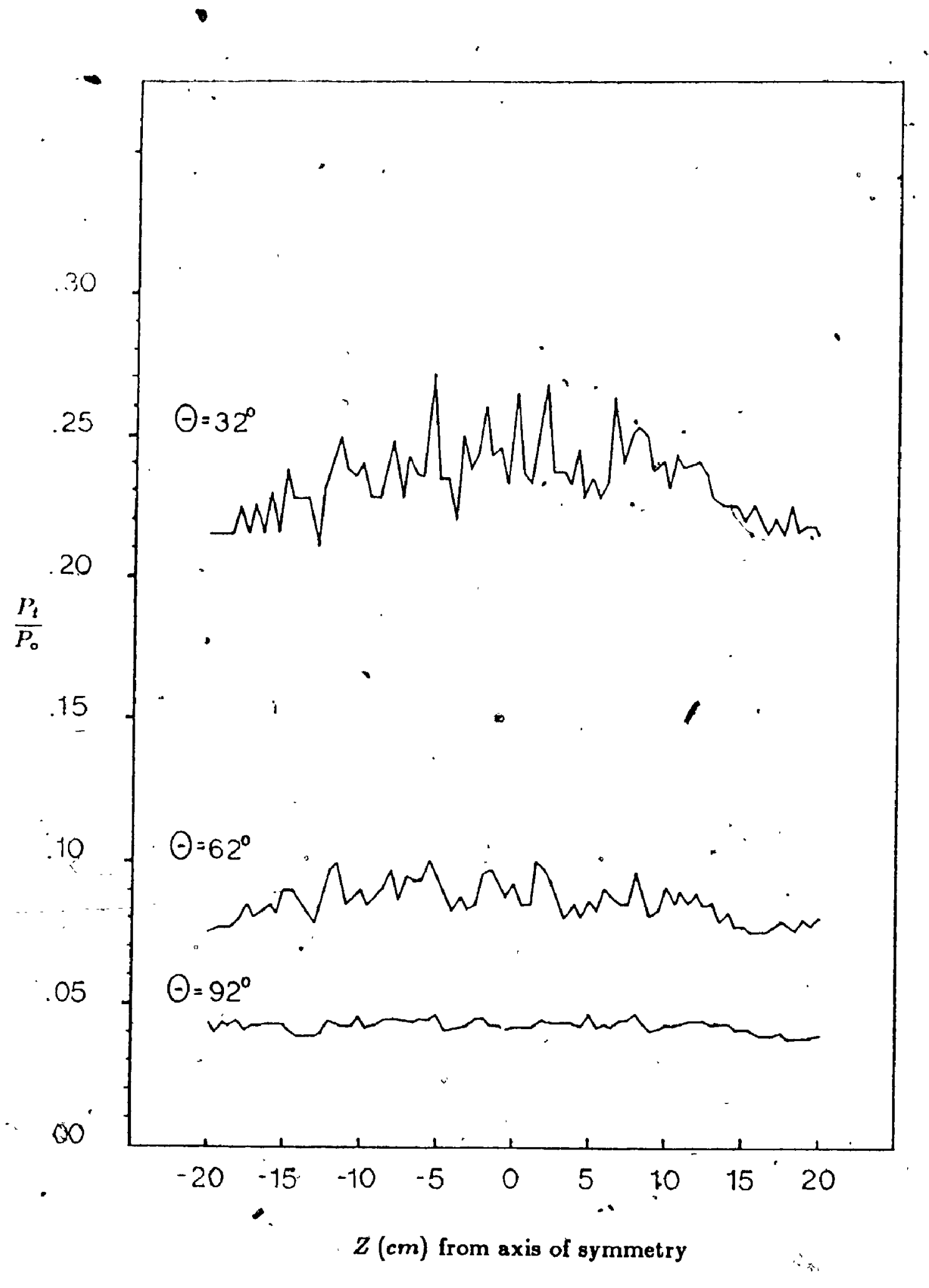

Figure 27: Spanwise Distribution of Maximum Total Pressure in Wall-Jet on Cylinder in Still Alr 


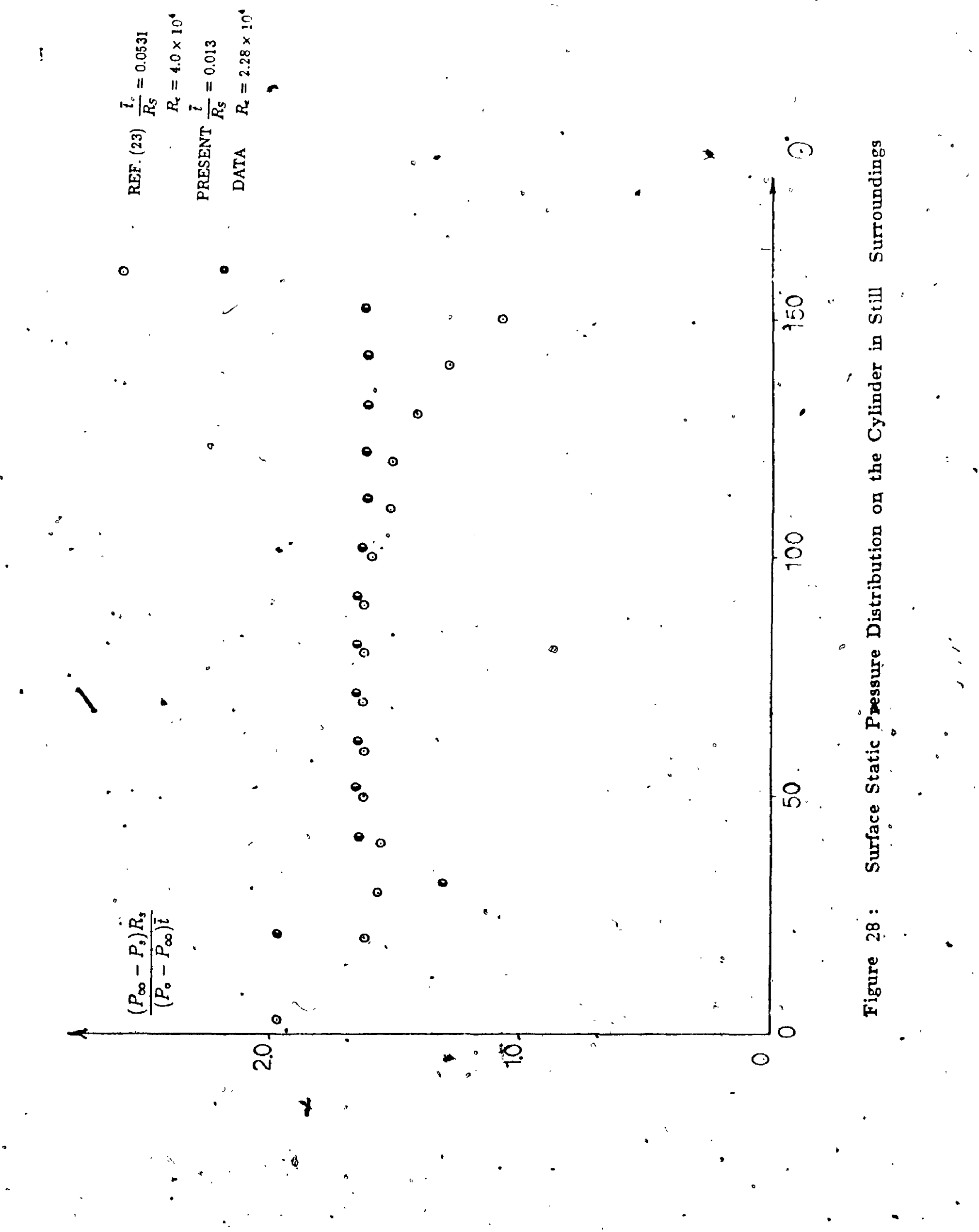




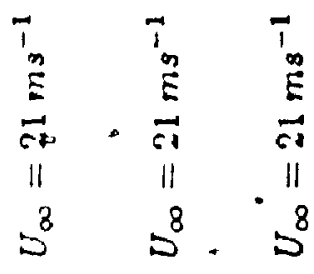

$\begin{array}{lll}\ddot{\Xi} & \ddot{\Xi} & \Xi \\ 0 & \| & \|\end{array}$

$u^{i}=u^{i} u^{i}$

$\begin{array}{ccc}\dot{0} & \dot{0} & \dot{0} \\ 0 & 0 & 0 \\ 11 & 11 & 11 \\ 0 & 0^{\dot{3}} & 0^{\dot{j}}-\end{array}$

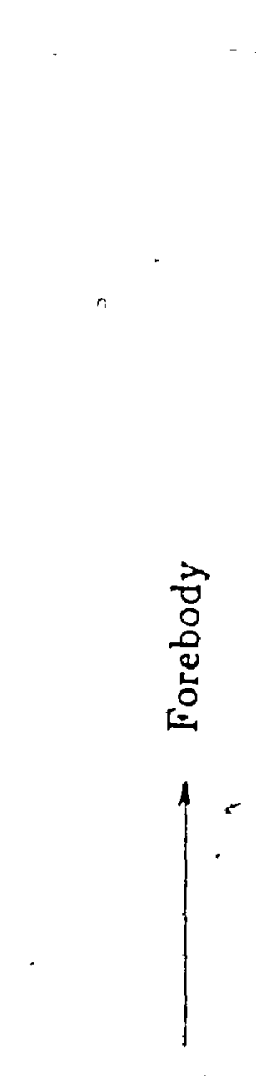

$\phi \uparrow . \phi$.
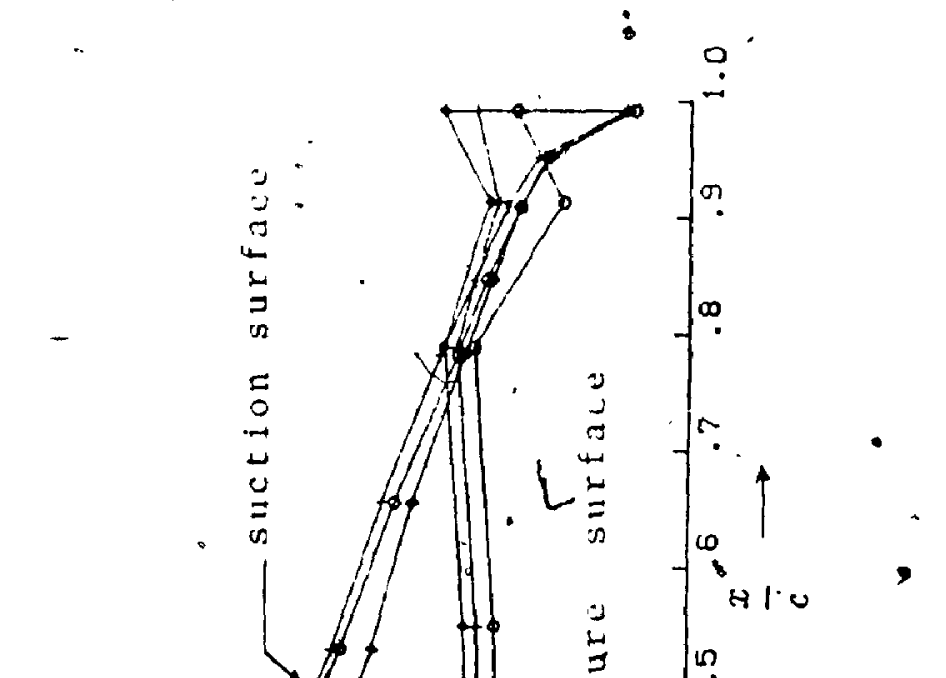

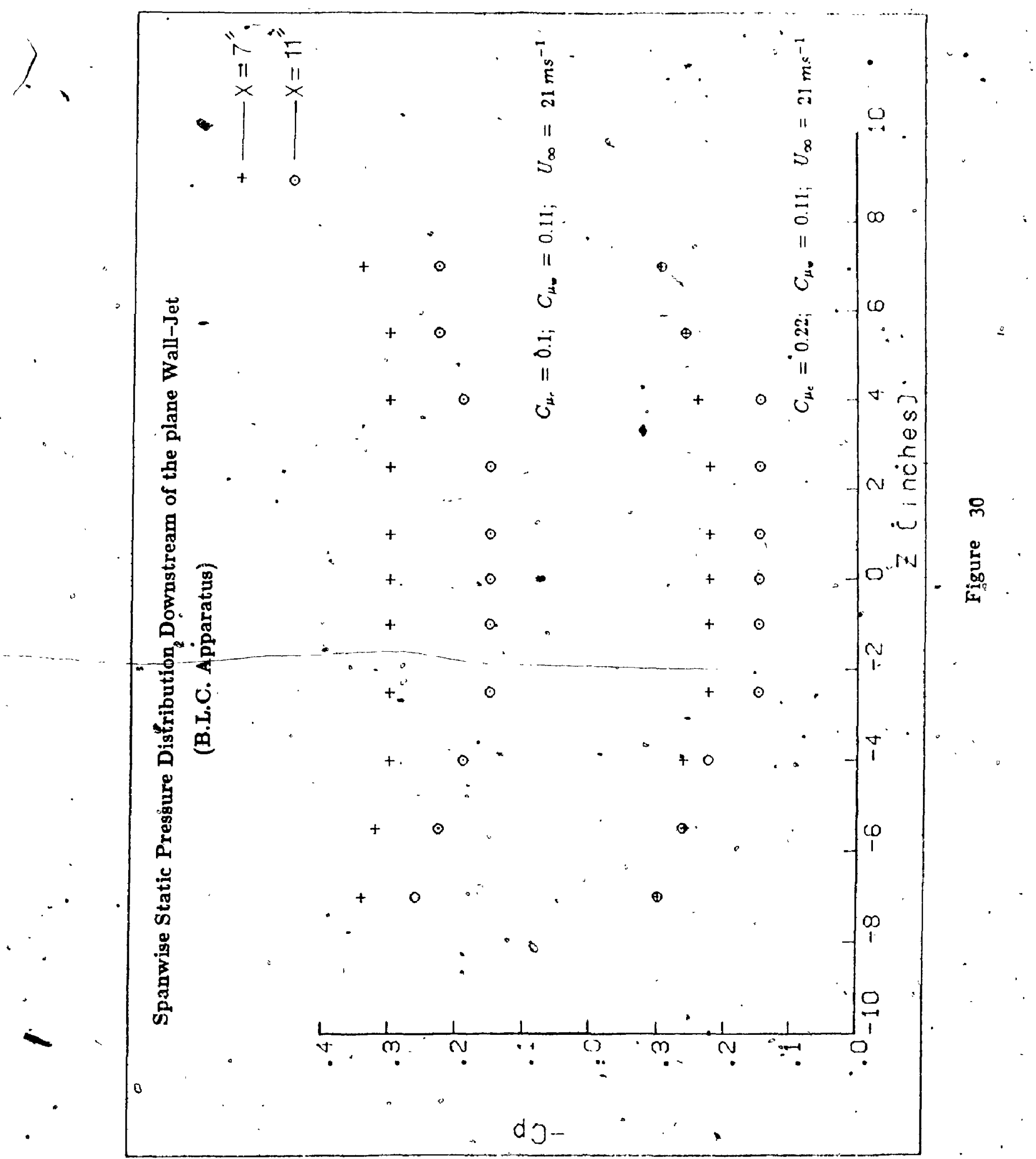



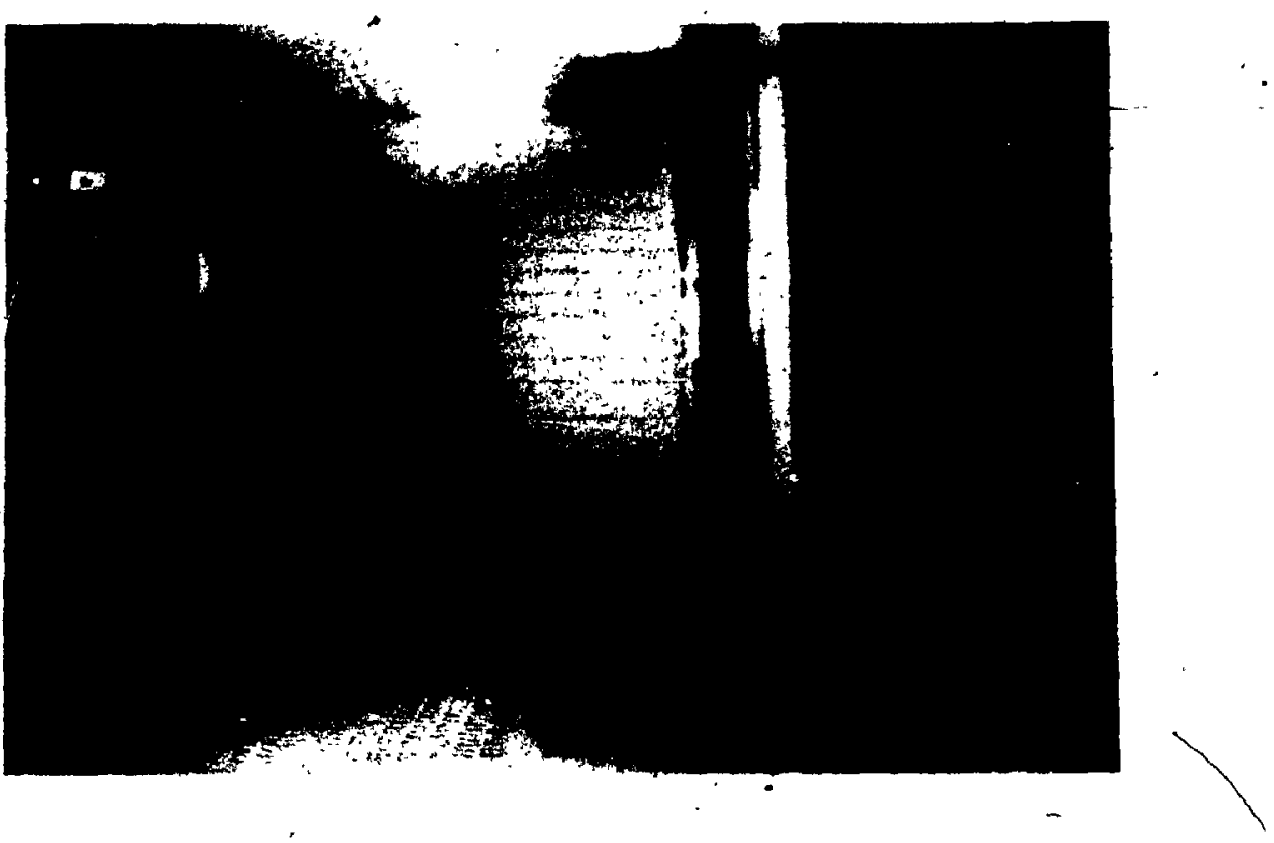

SUCTION OFF

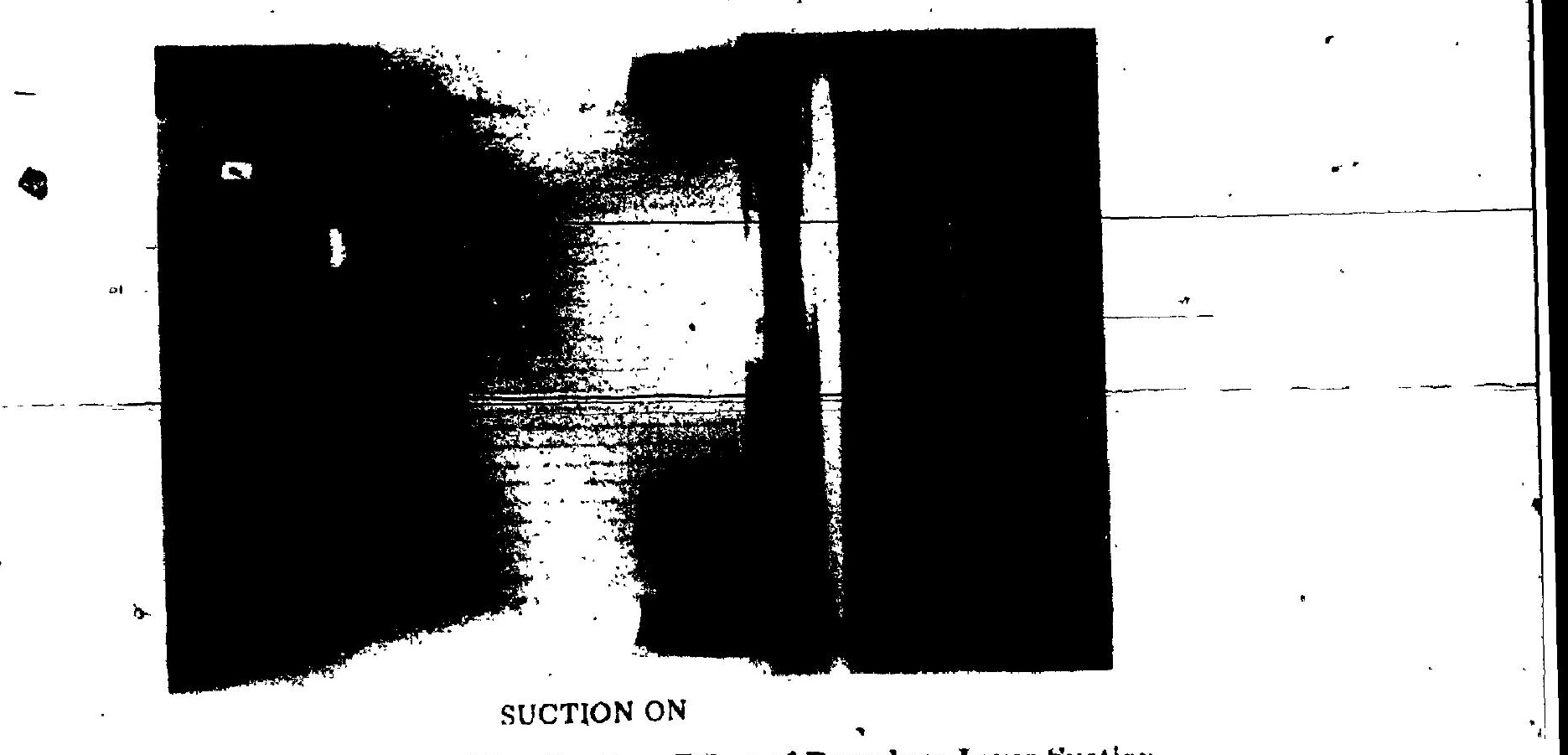

Figur. 32 : Flow Vlaualization, Effect of Boundary Layer Suction $C_{\mu}=020, \quad C_{\mu_{-}}, 011 ; U_{\infty}-21 \mathrm{~ms} \cdot$ 


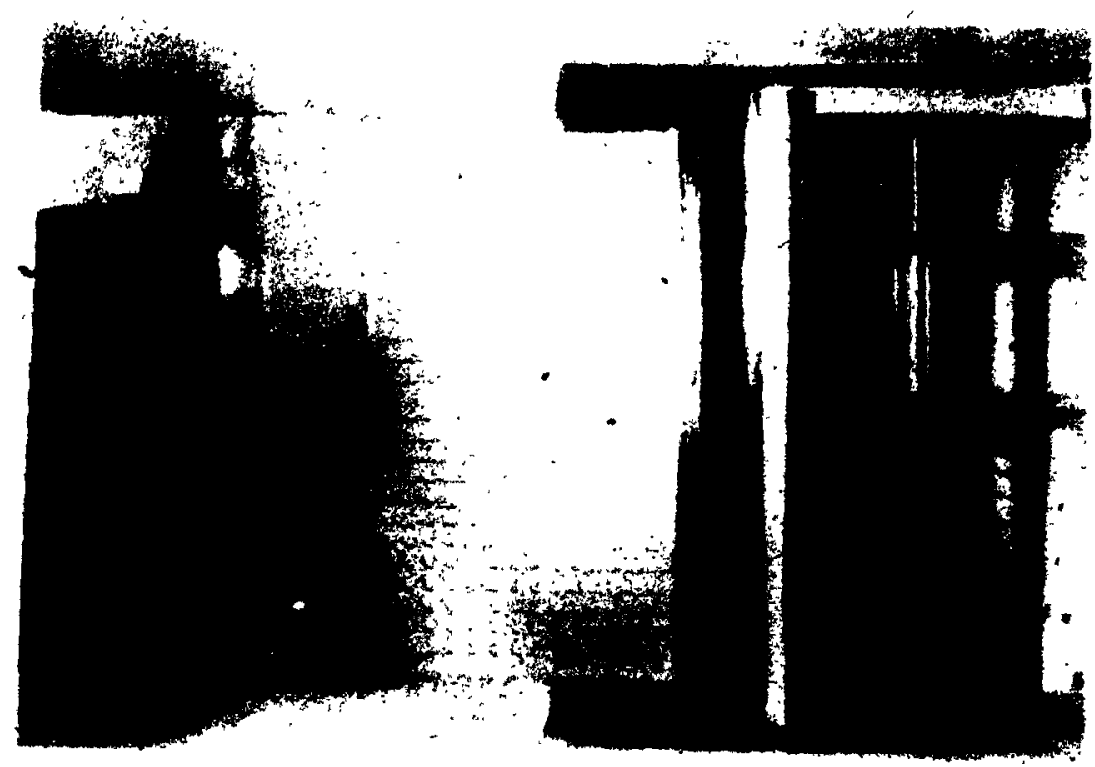

SUCTION OFF

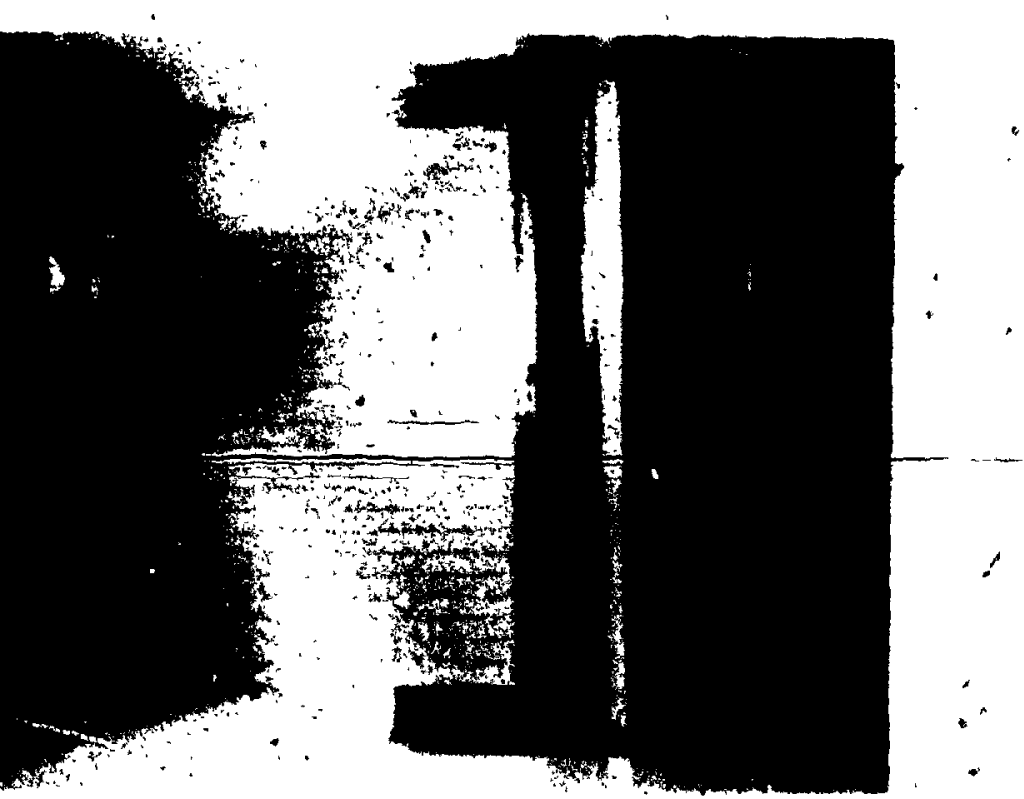

SUCTION ON

Figurv 33 : Flow Visualization, Effect of Boundary Lnyor Sirction

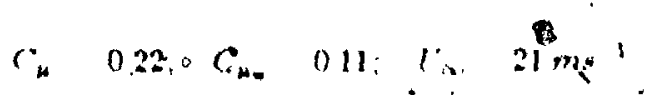




$$
E
$$


$\gamma$

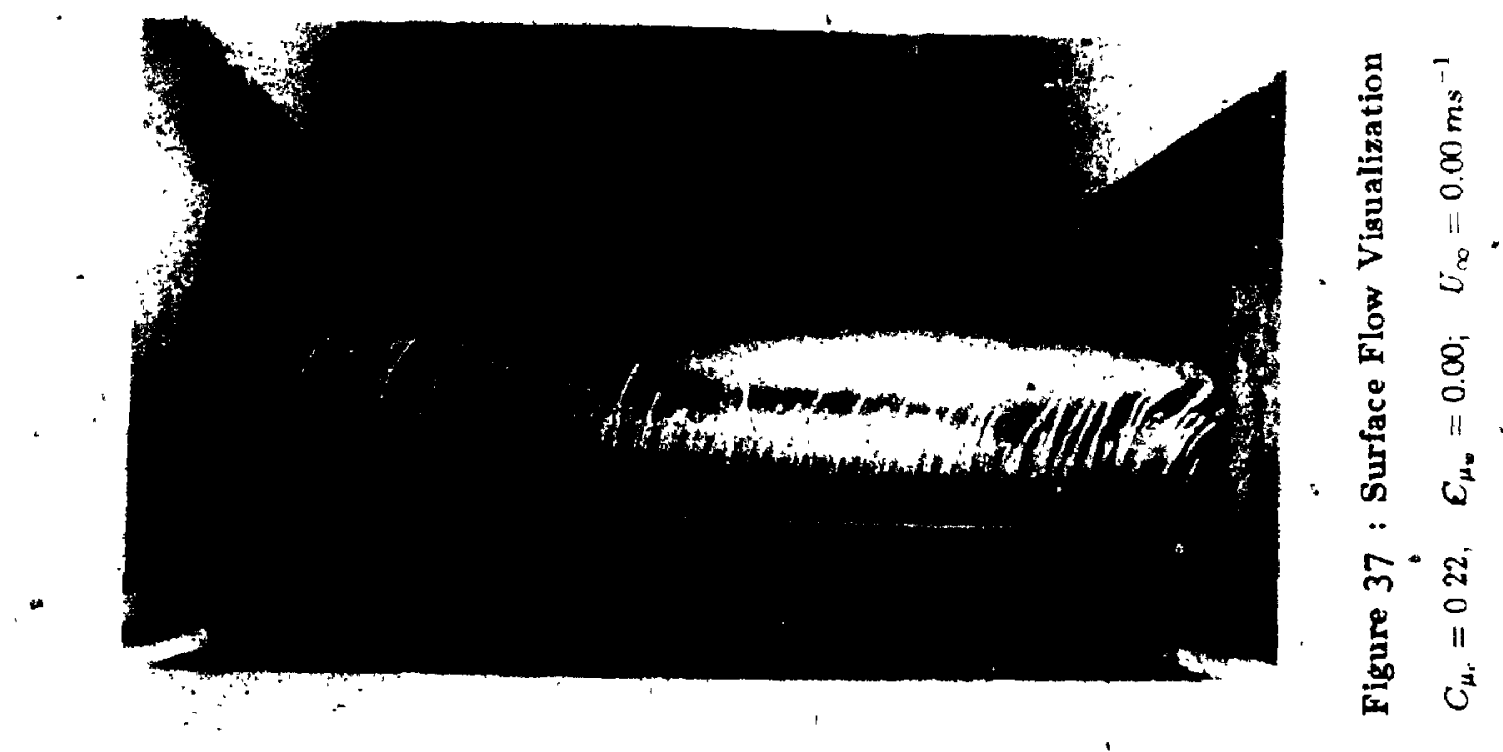

4

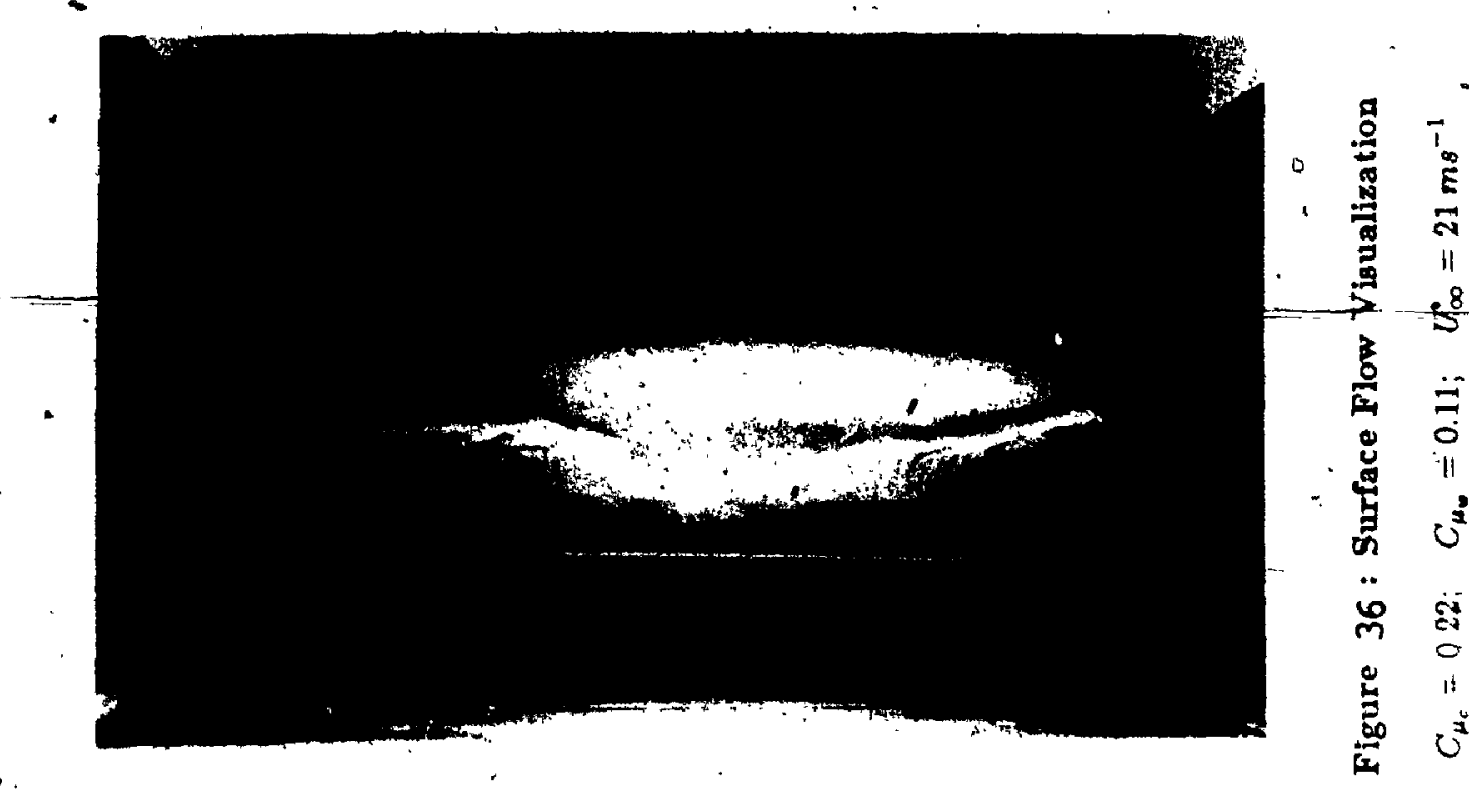




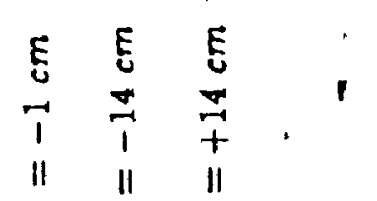

$$
\begin{aligned}
& \text { N. N N. iั } \\
& \odot<+
\end{aligned}
$$

5
I
11

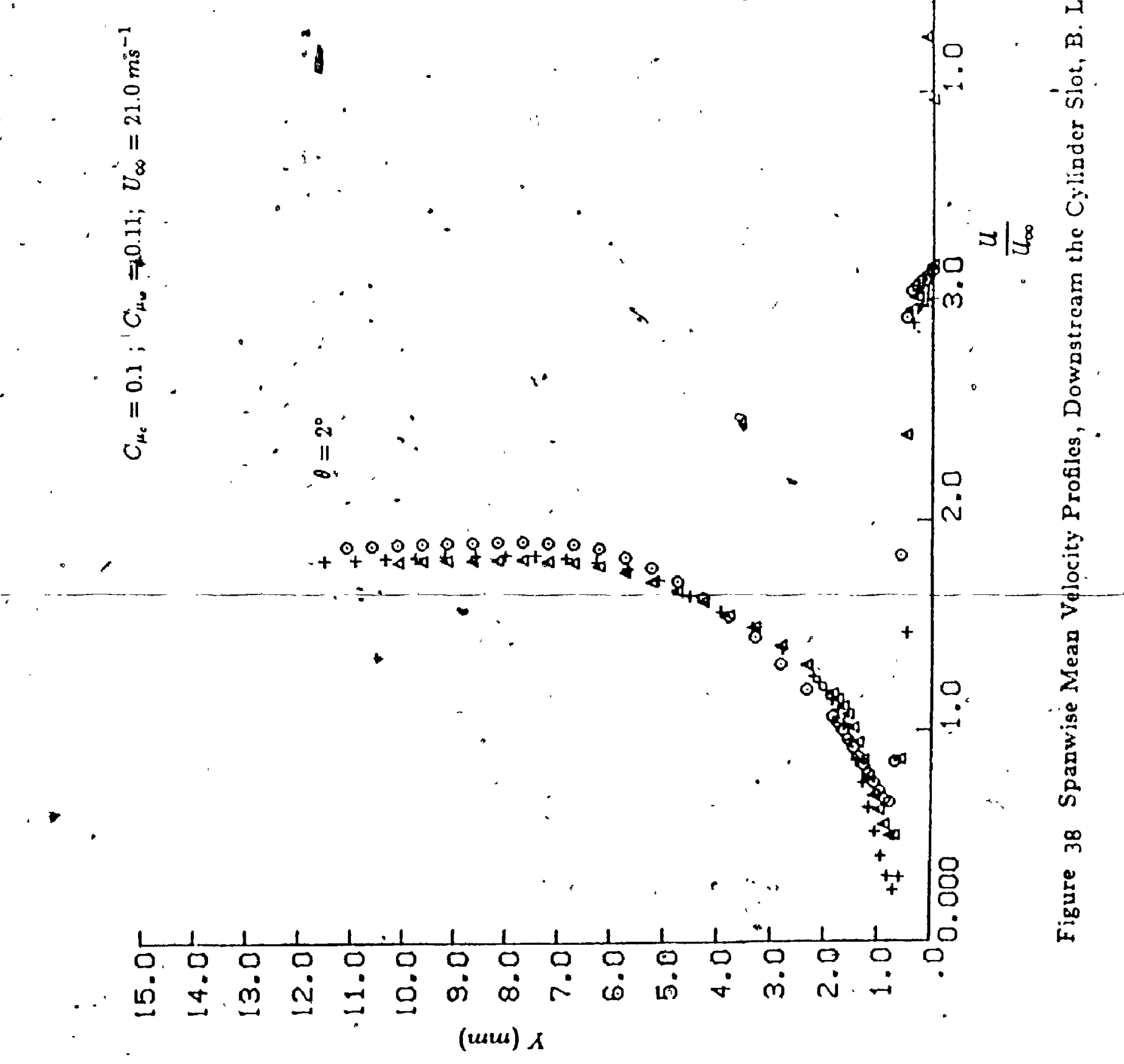




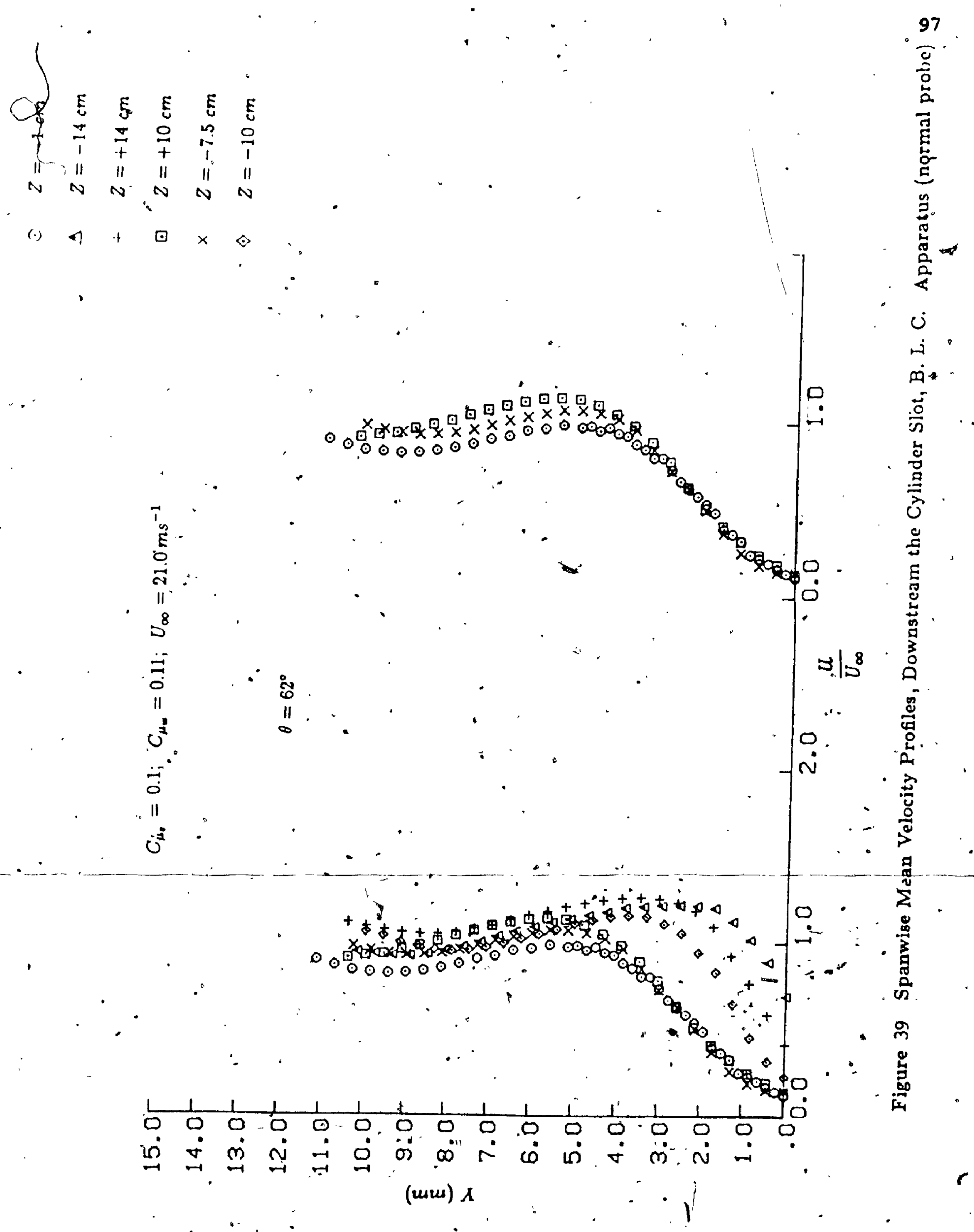




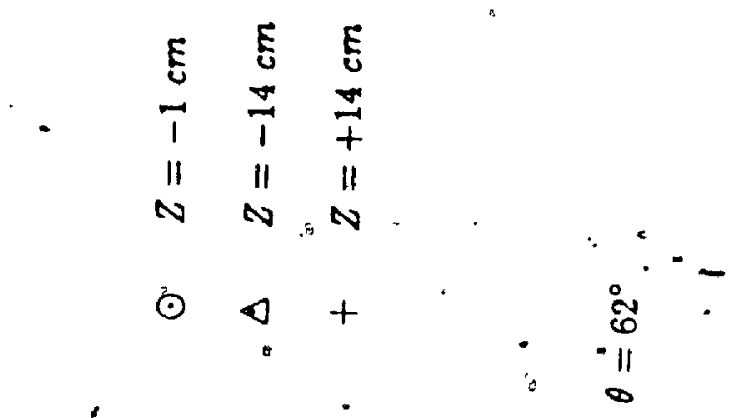

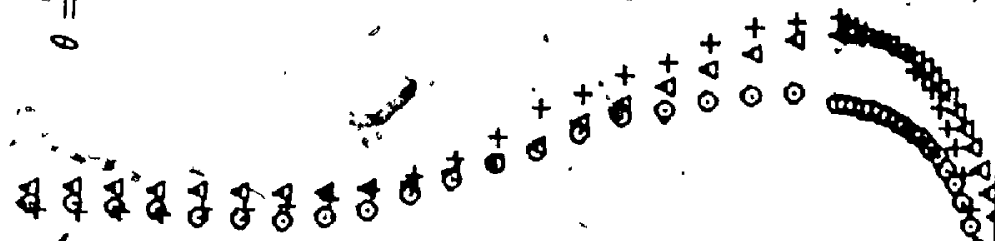
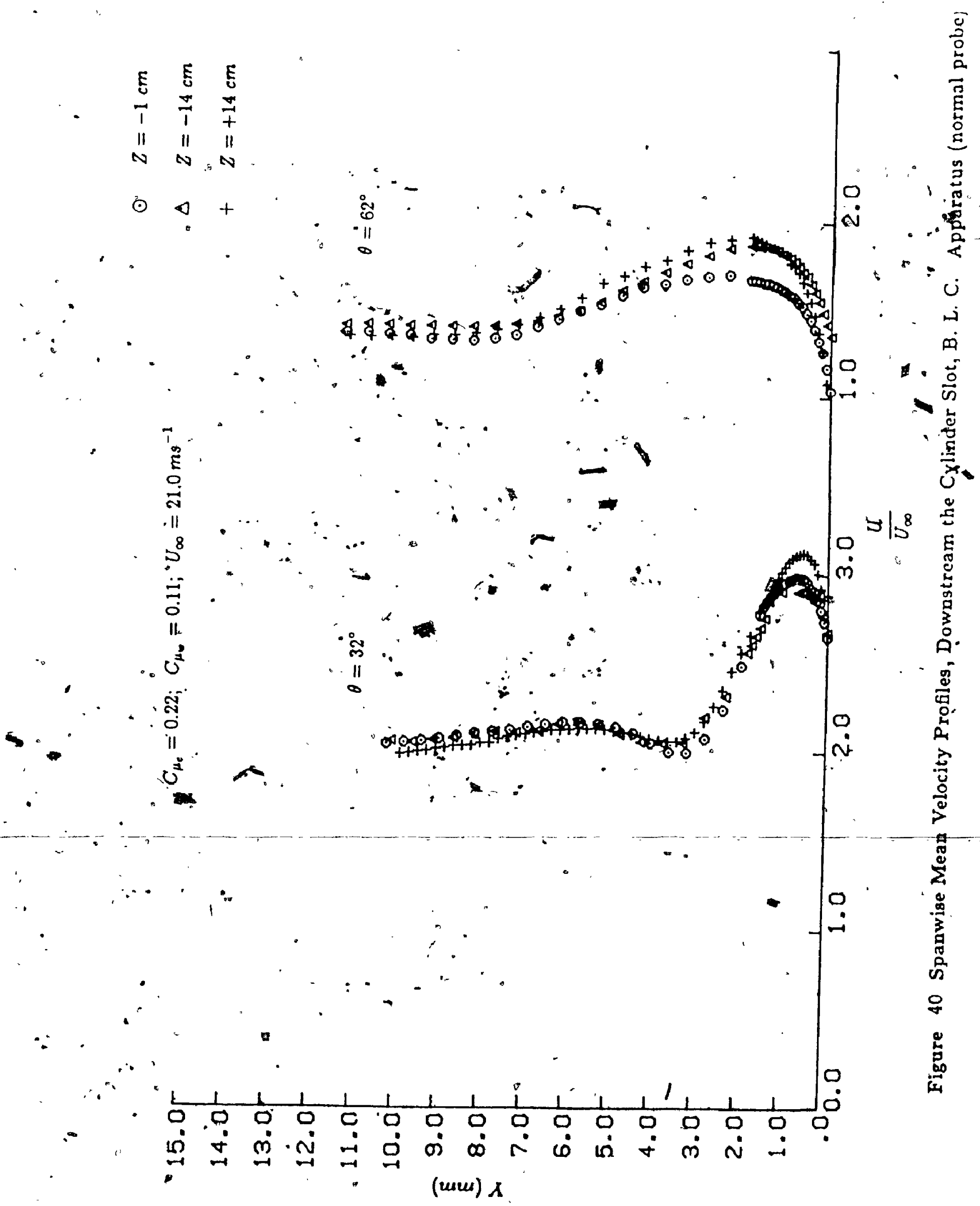


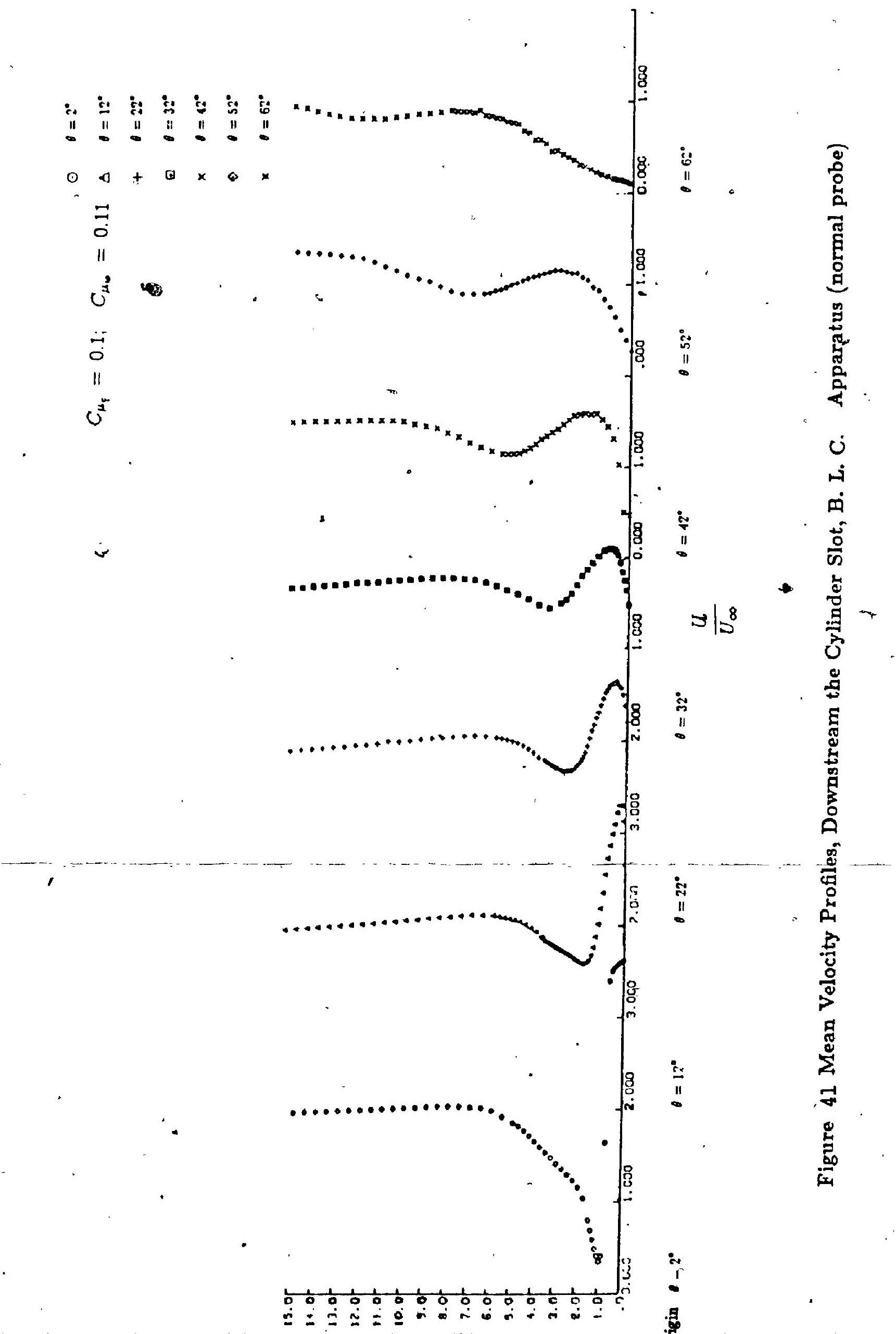




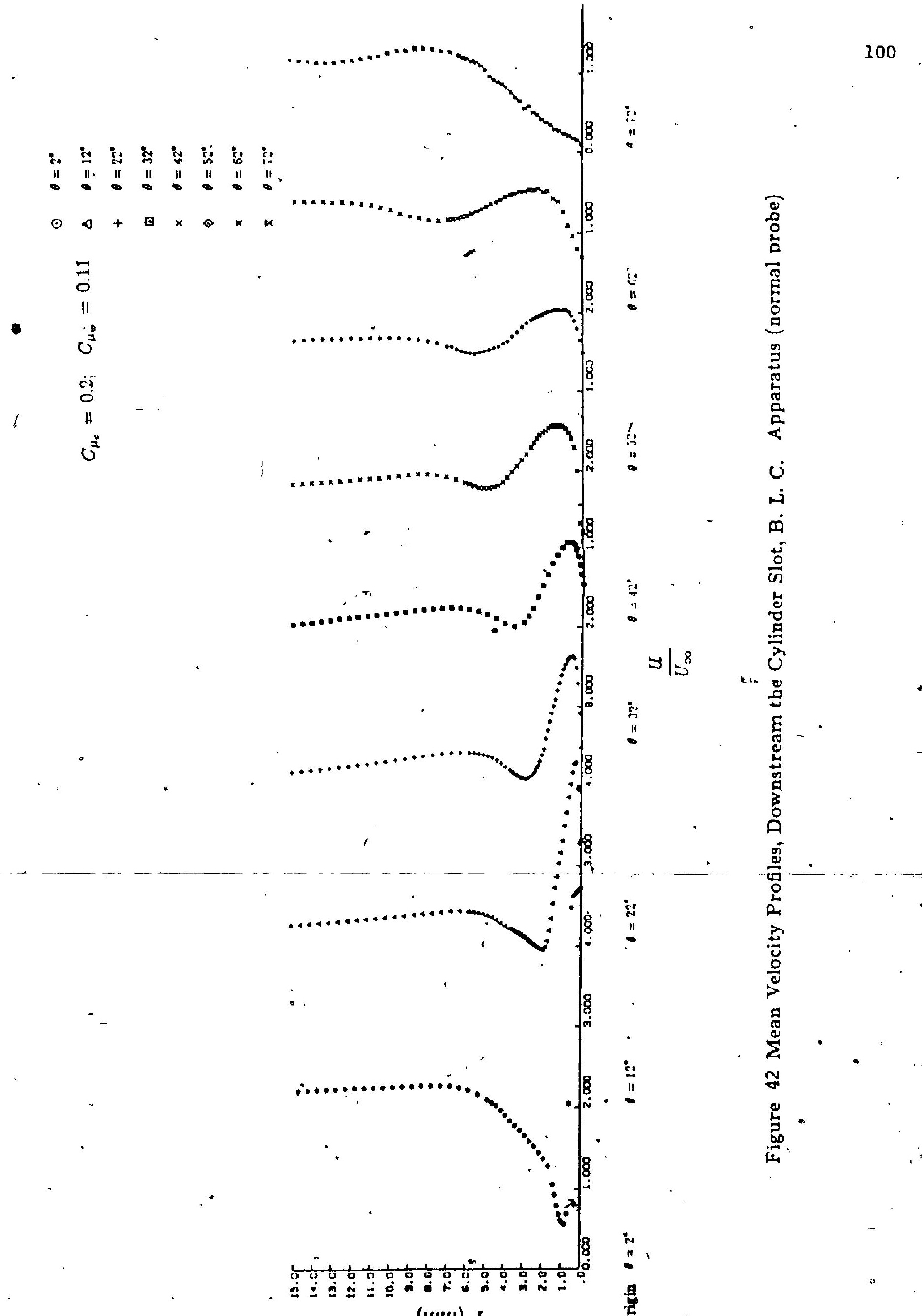




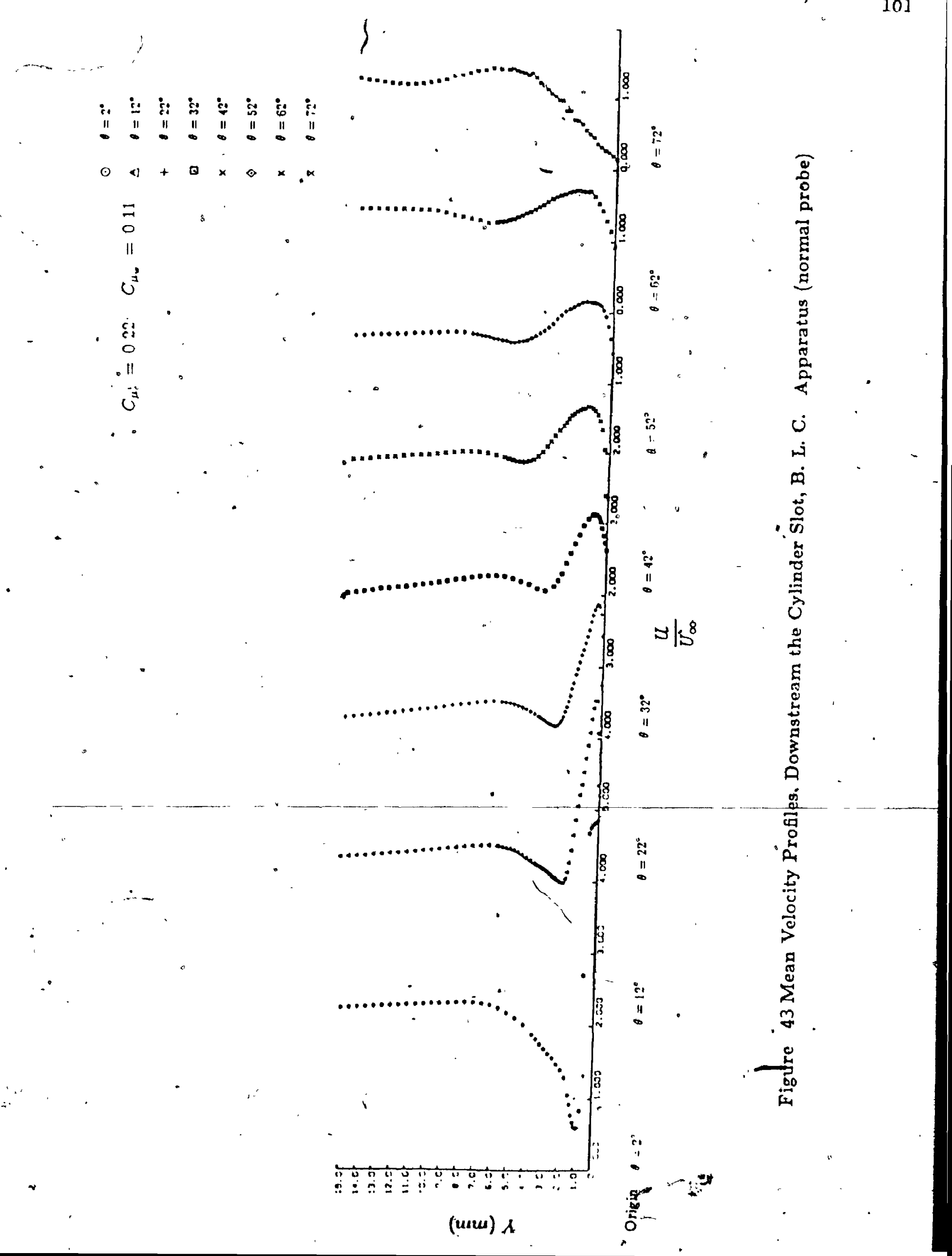




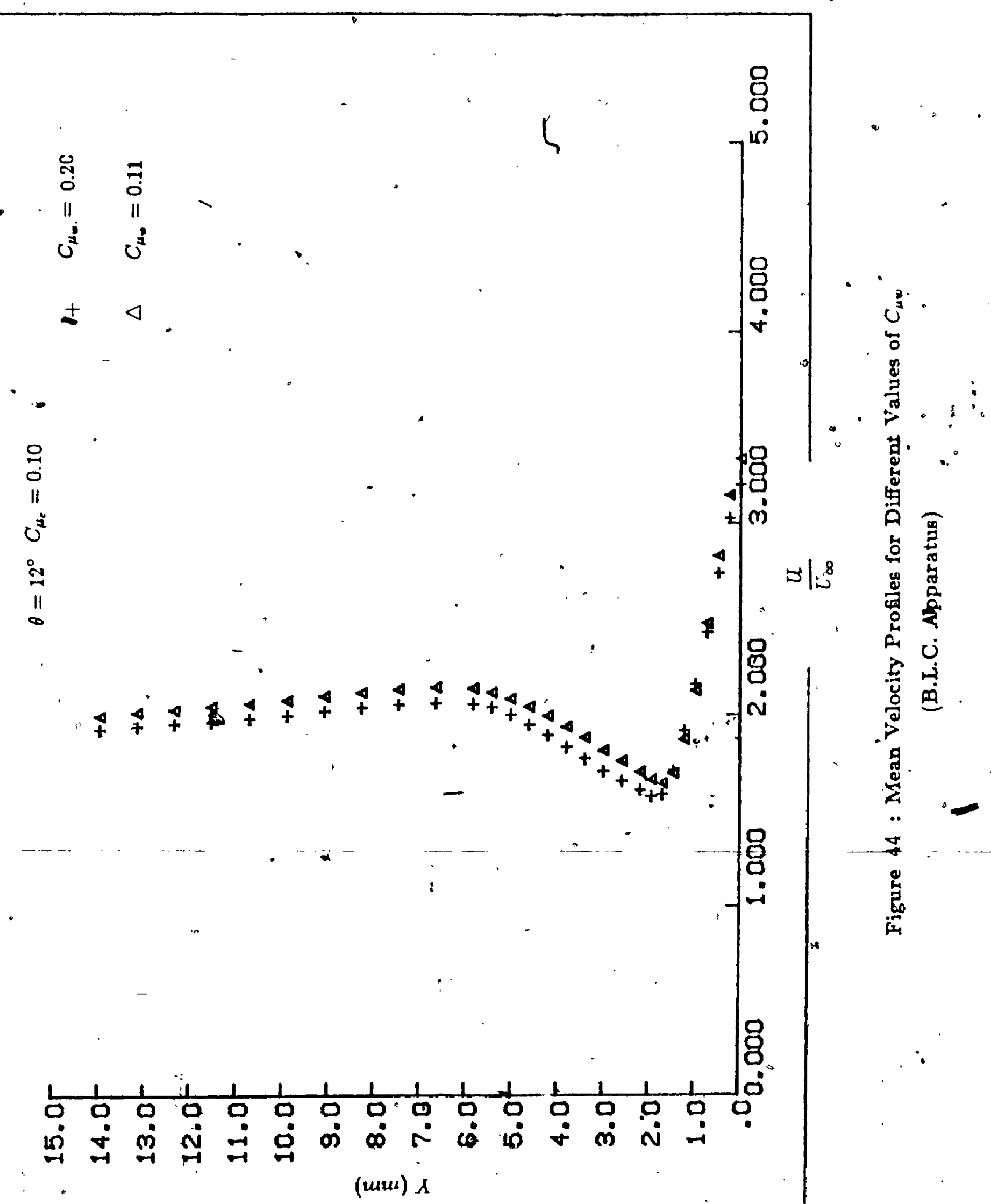




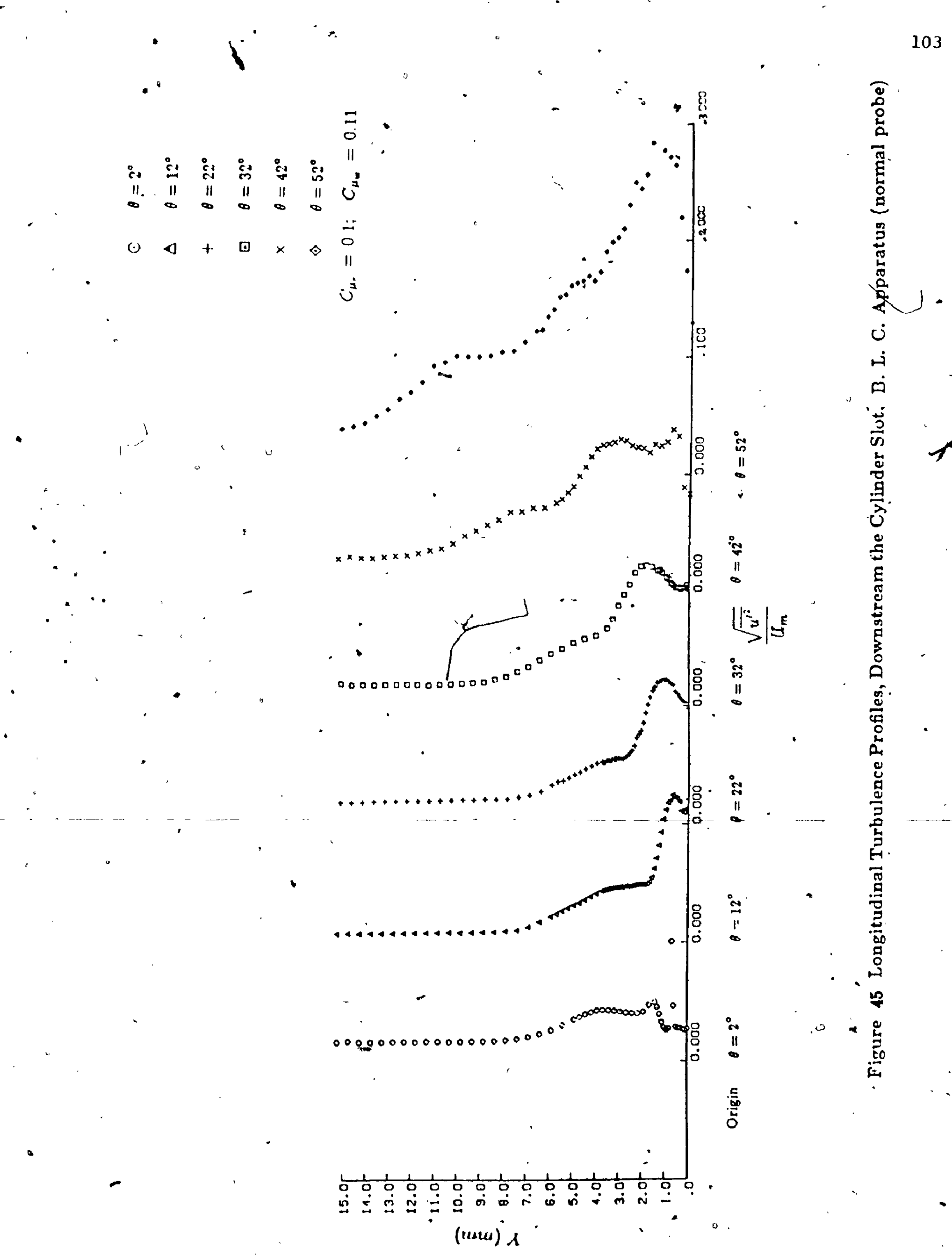


0

อ

$11=$
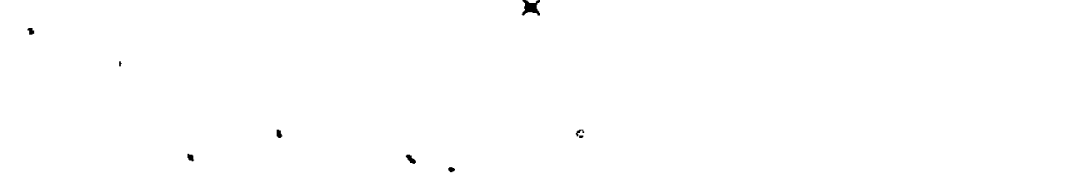

1
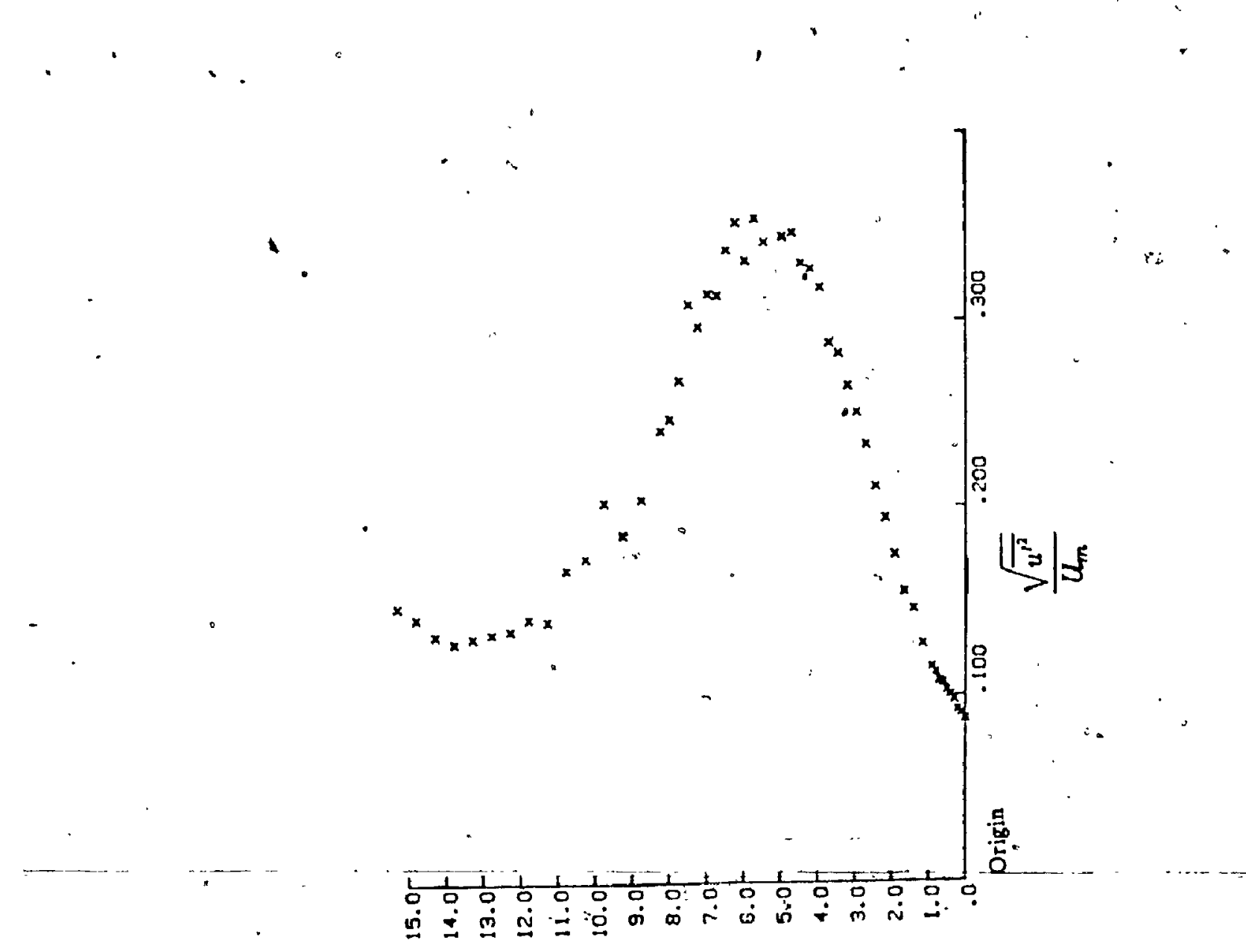

"(uाк) $\lambda$ 


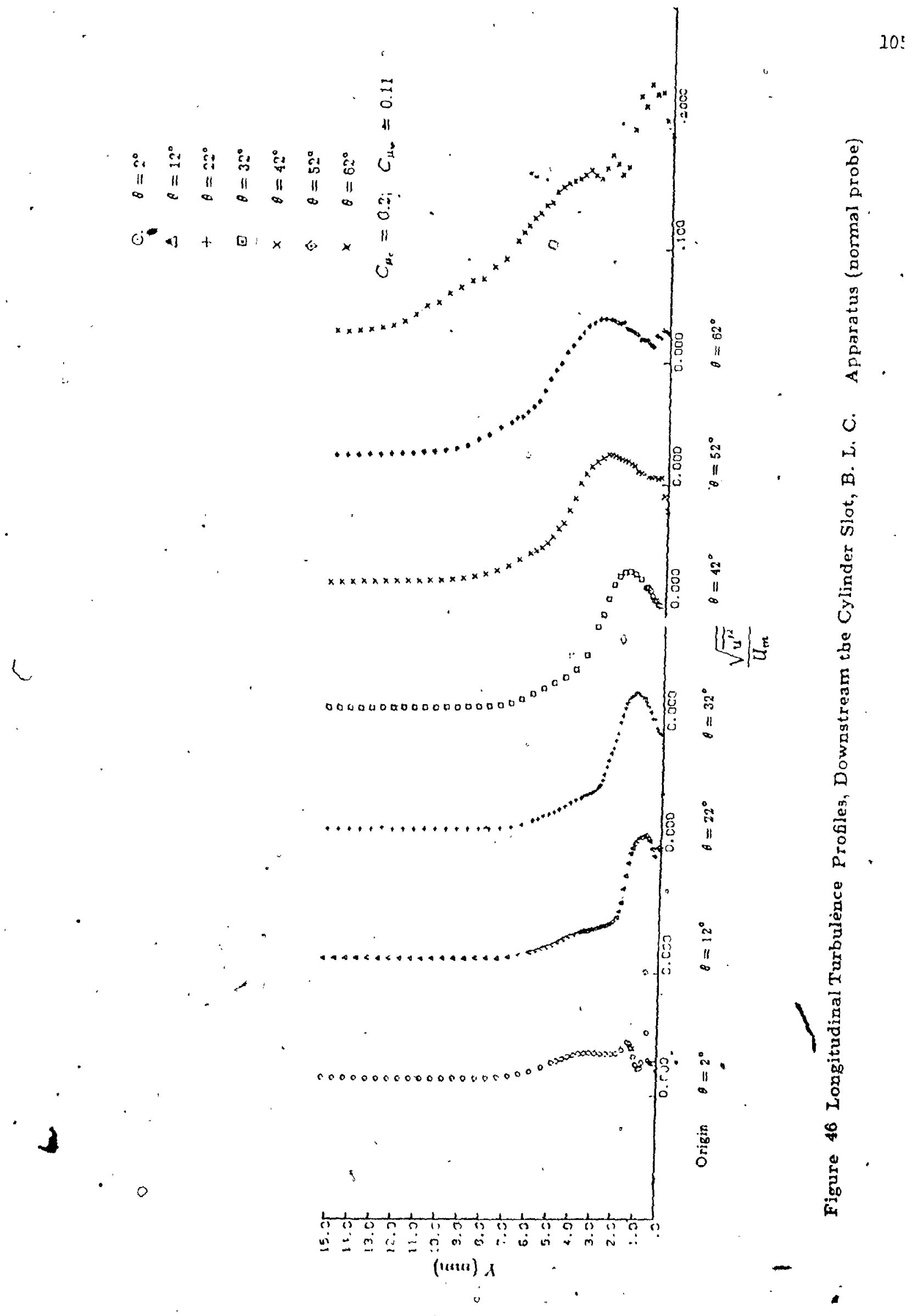




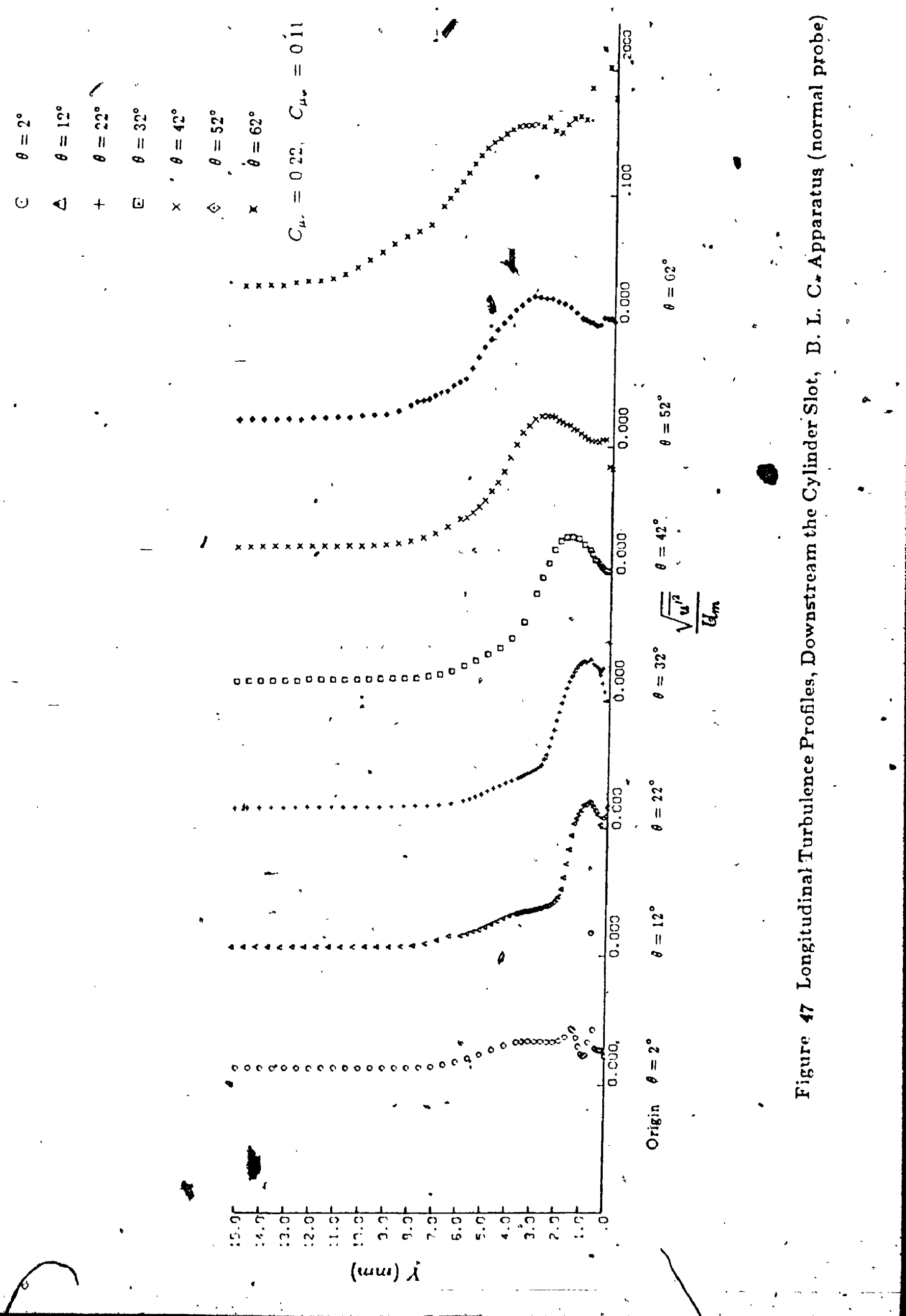




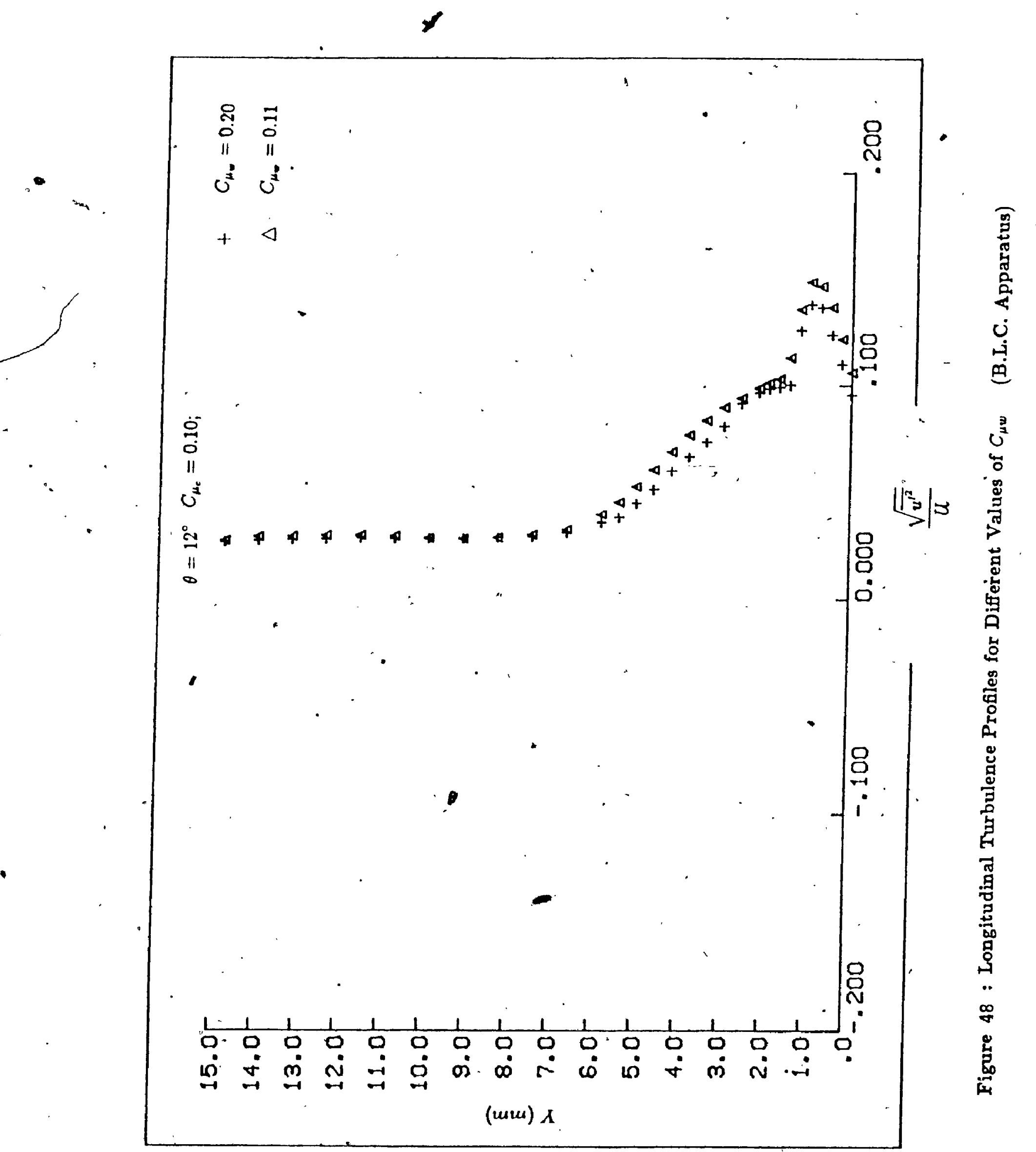




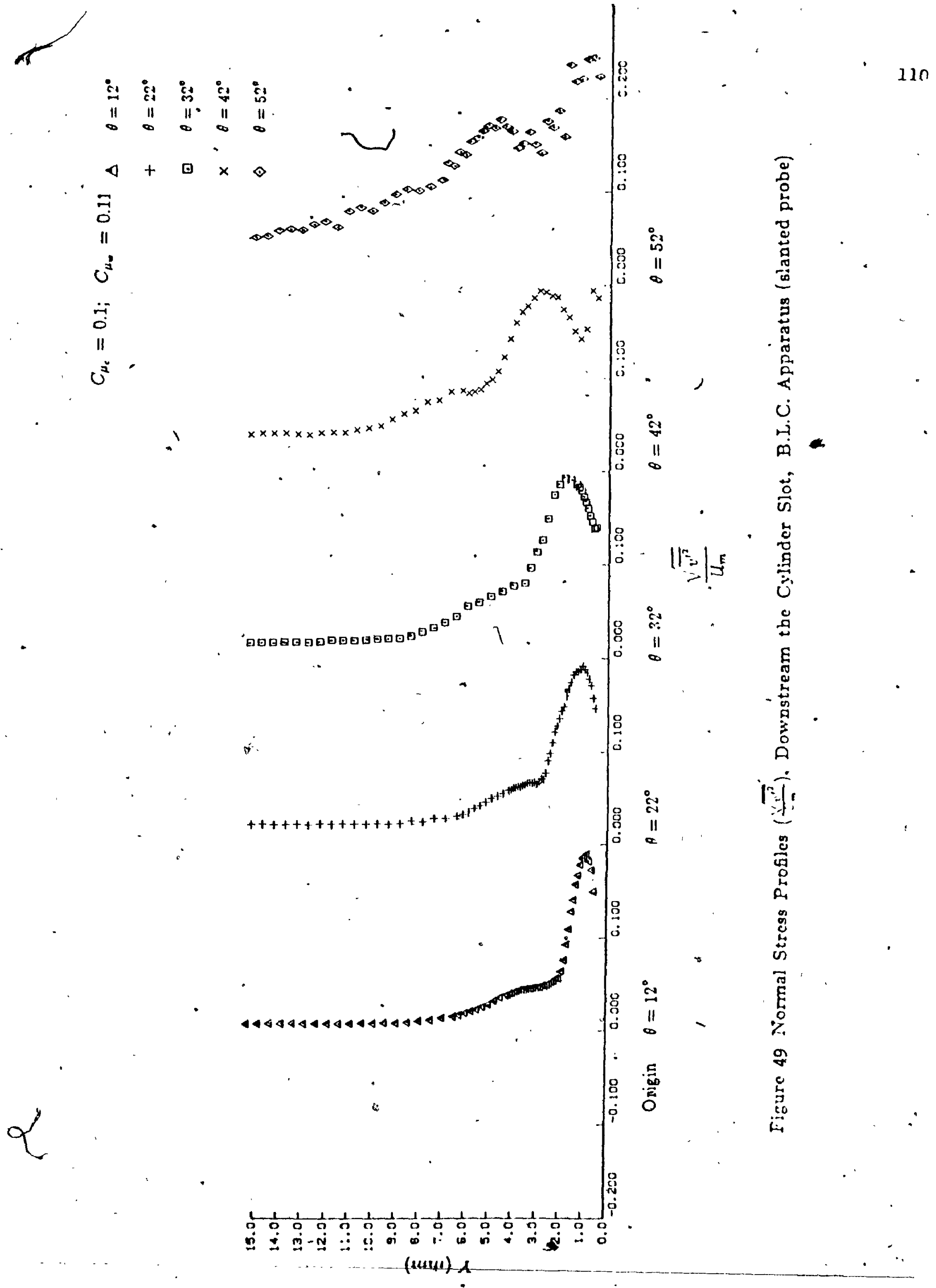




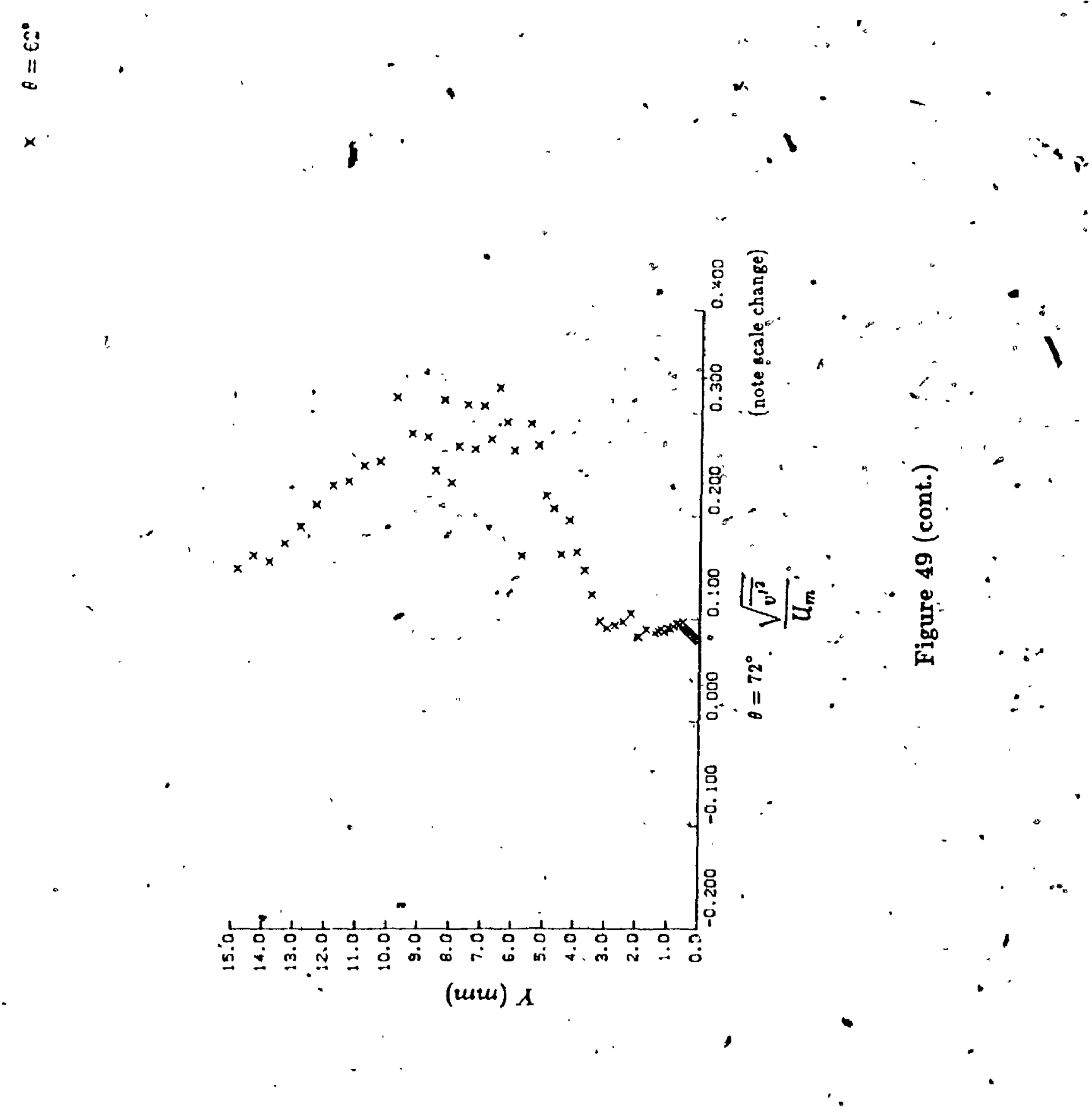




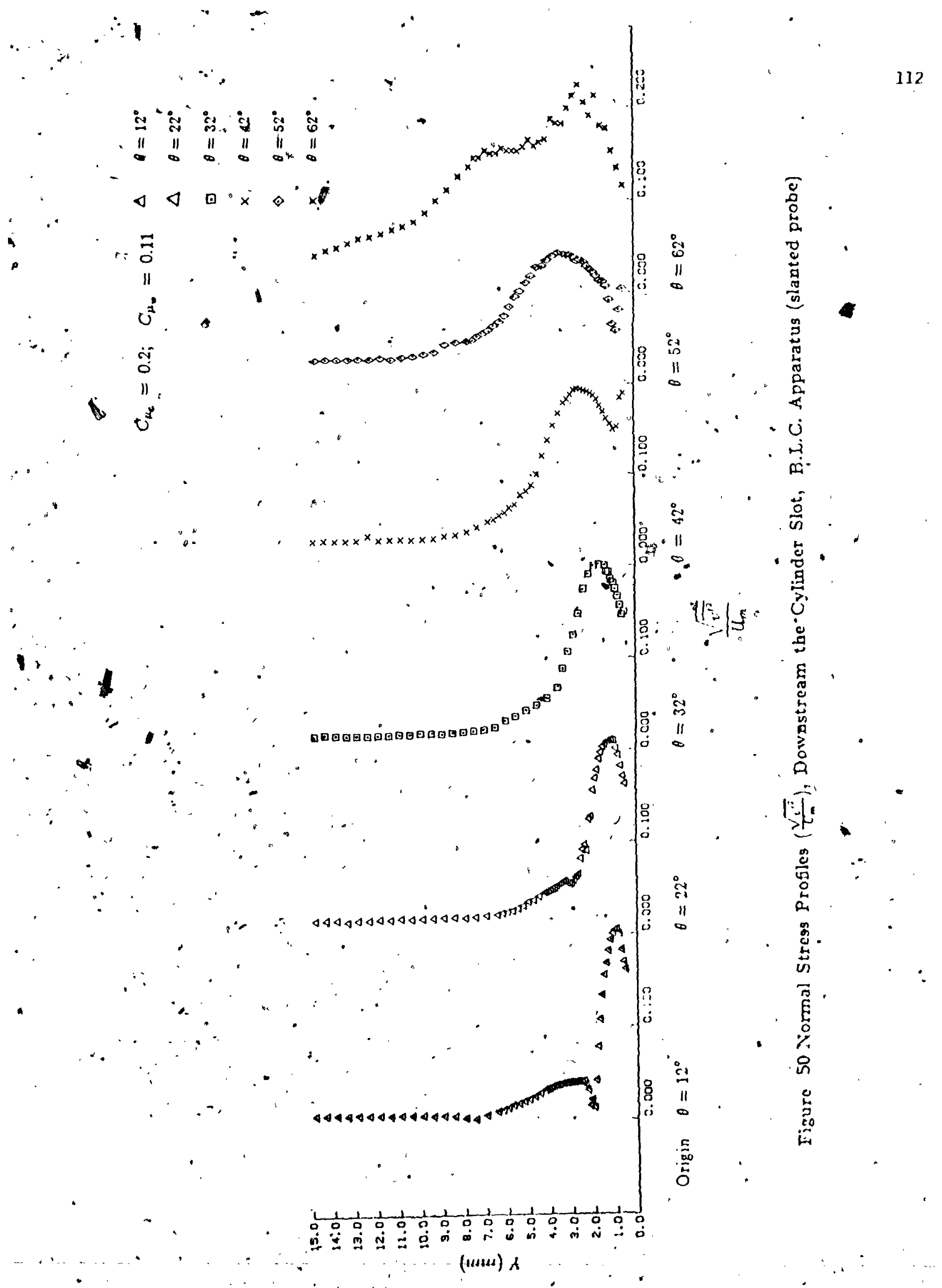



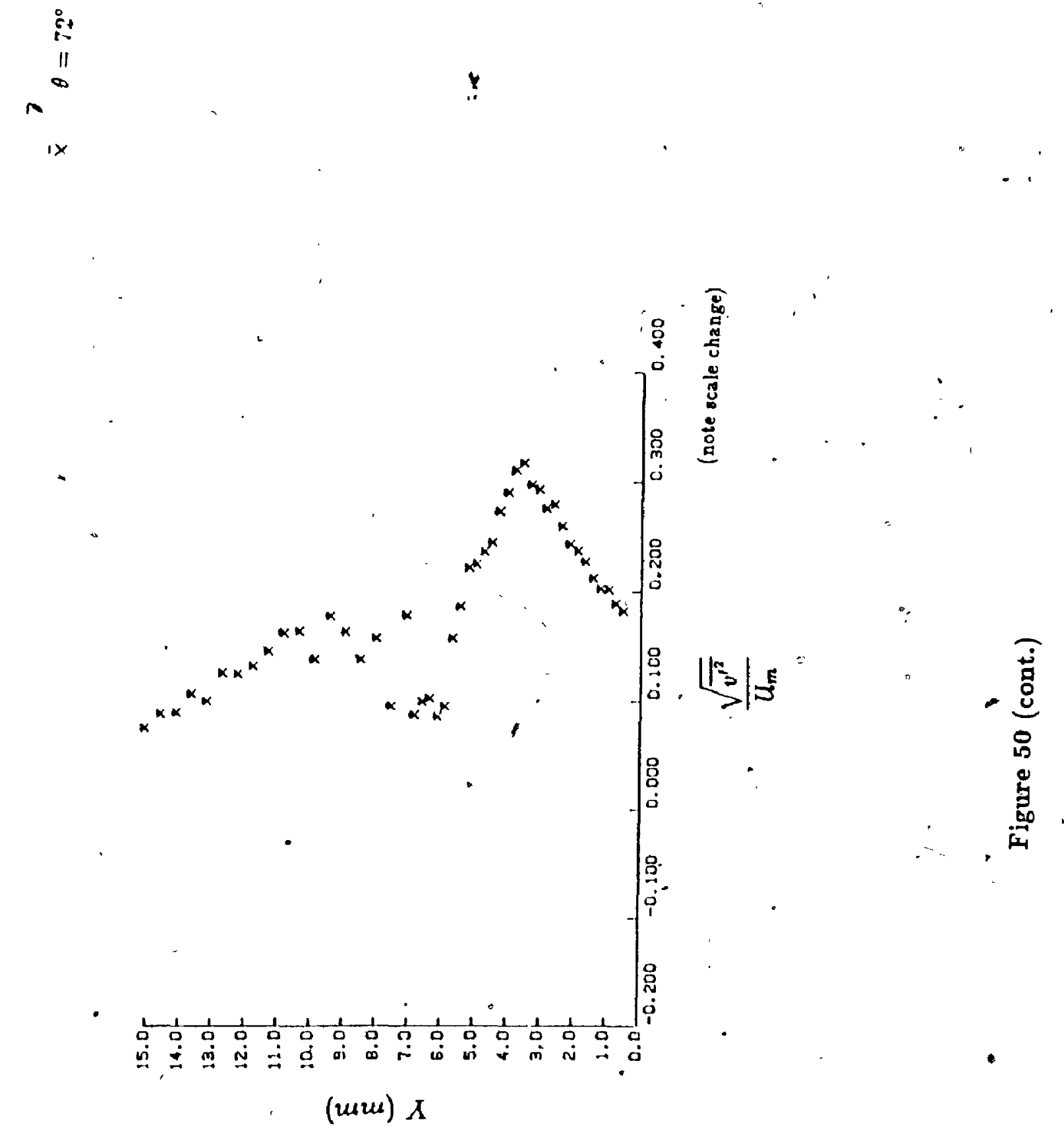


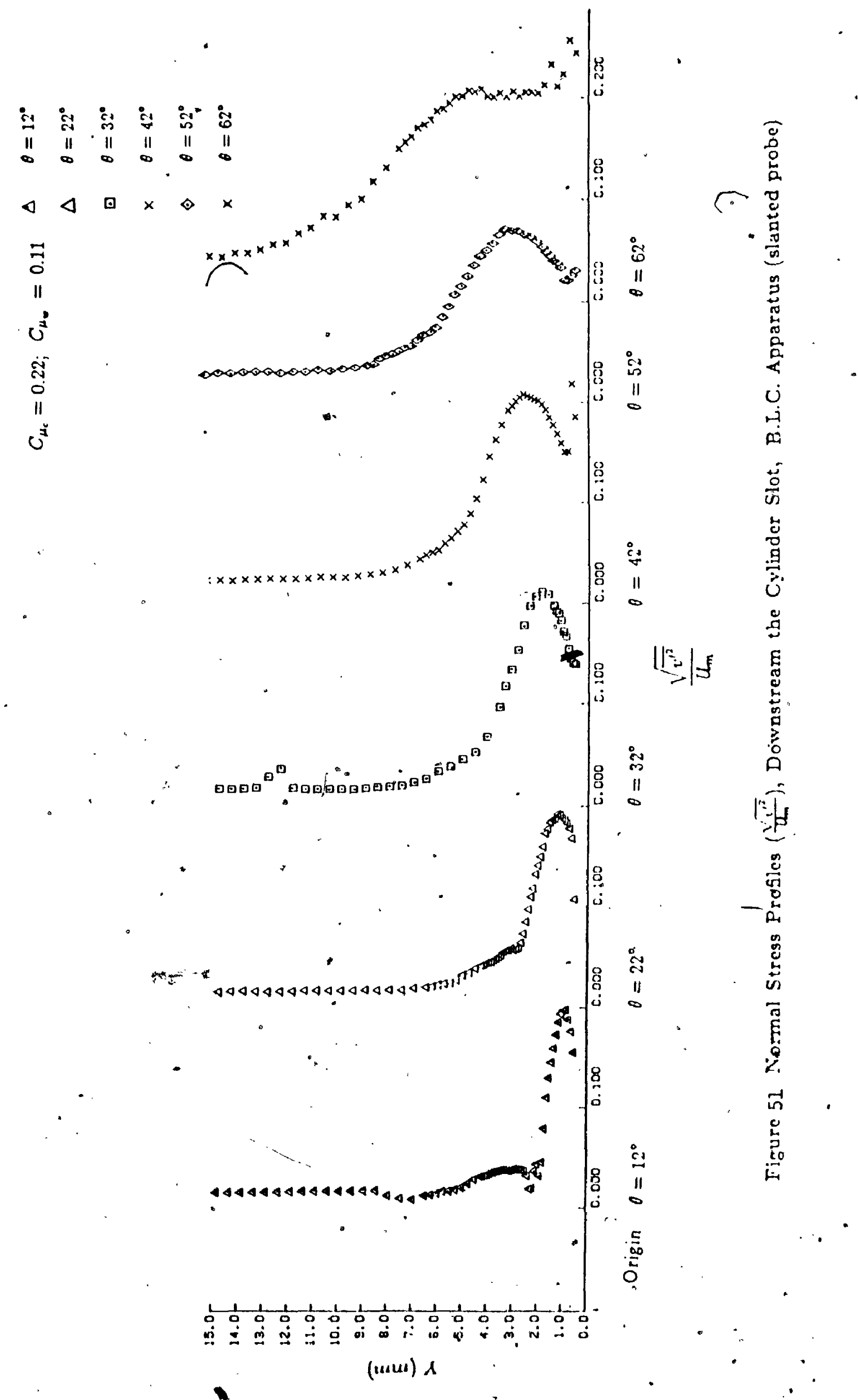



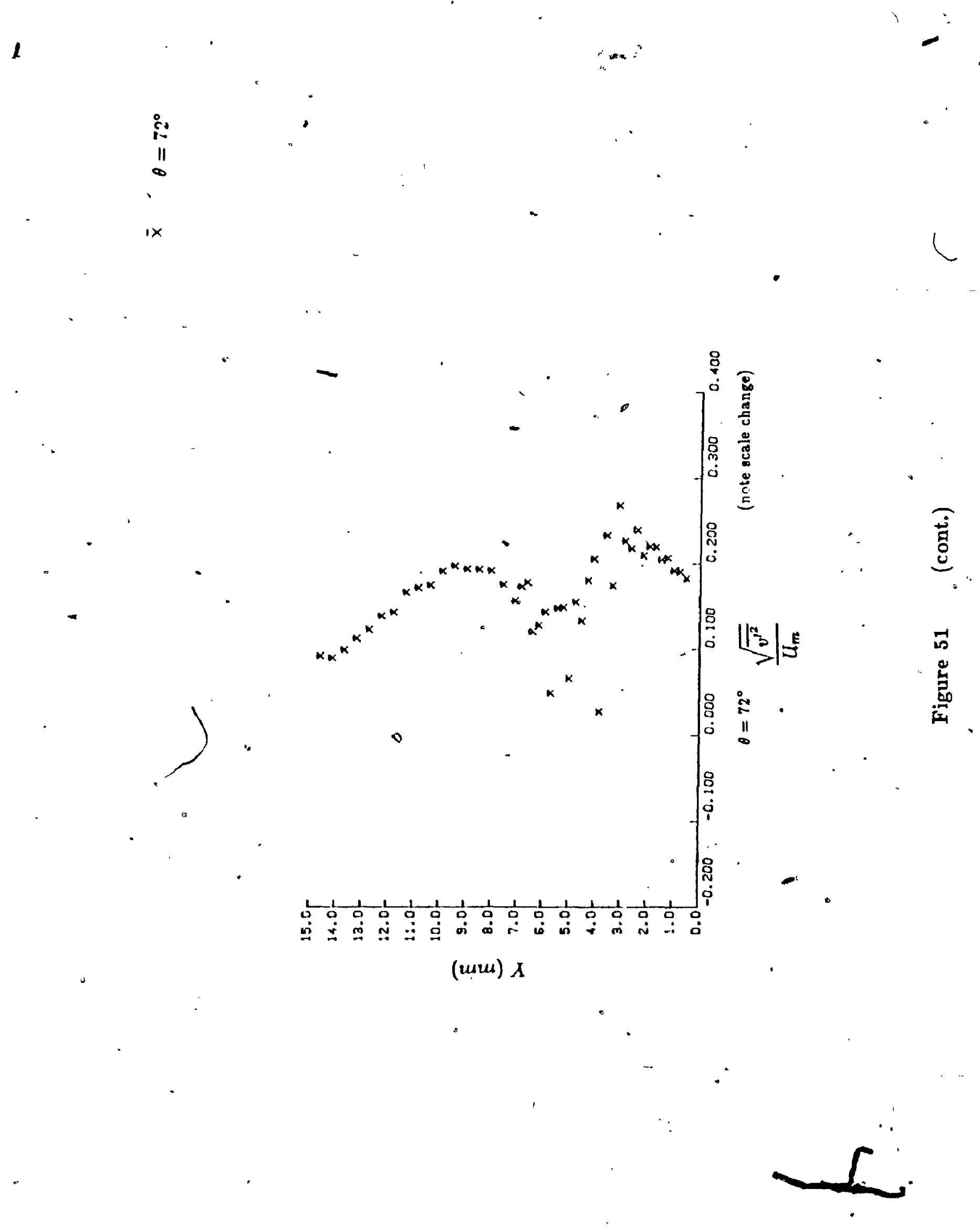


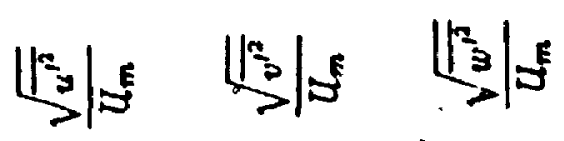

i

ב.

$u^{11}$

- ت্்

$\bullet^{.}$

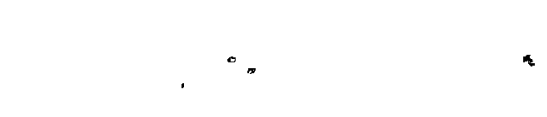

II

$u^{i}$

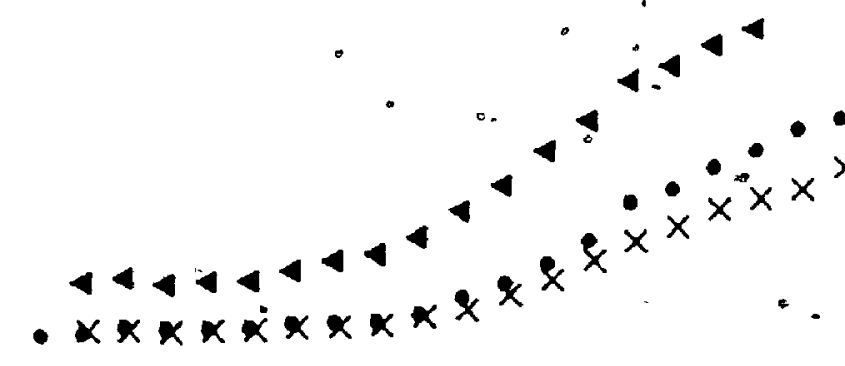

ำ

$-11$

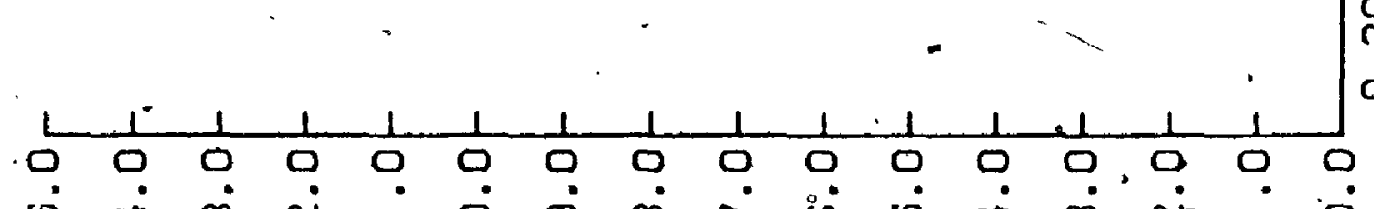

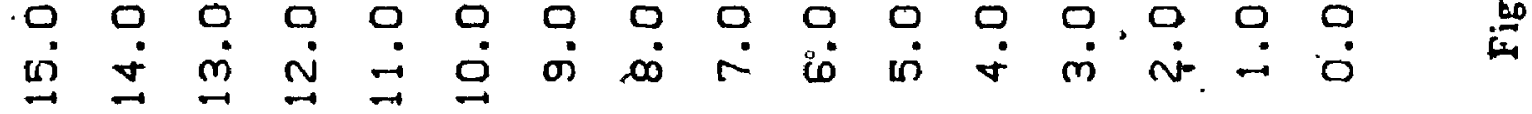

[usu ] 


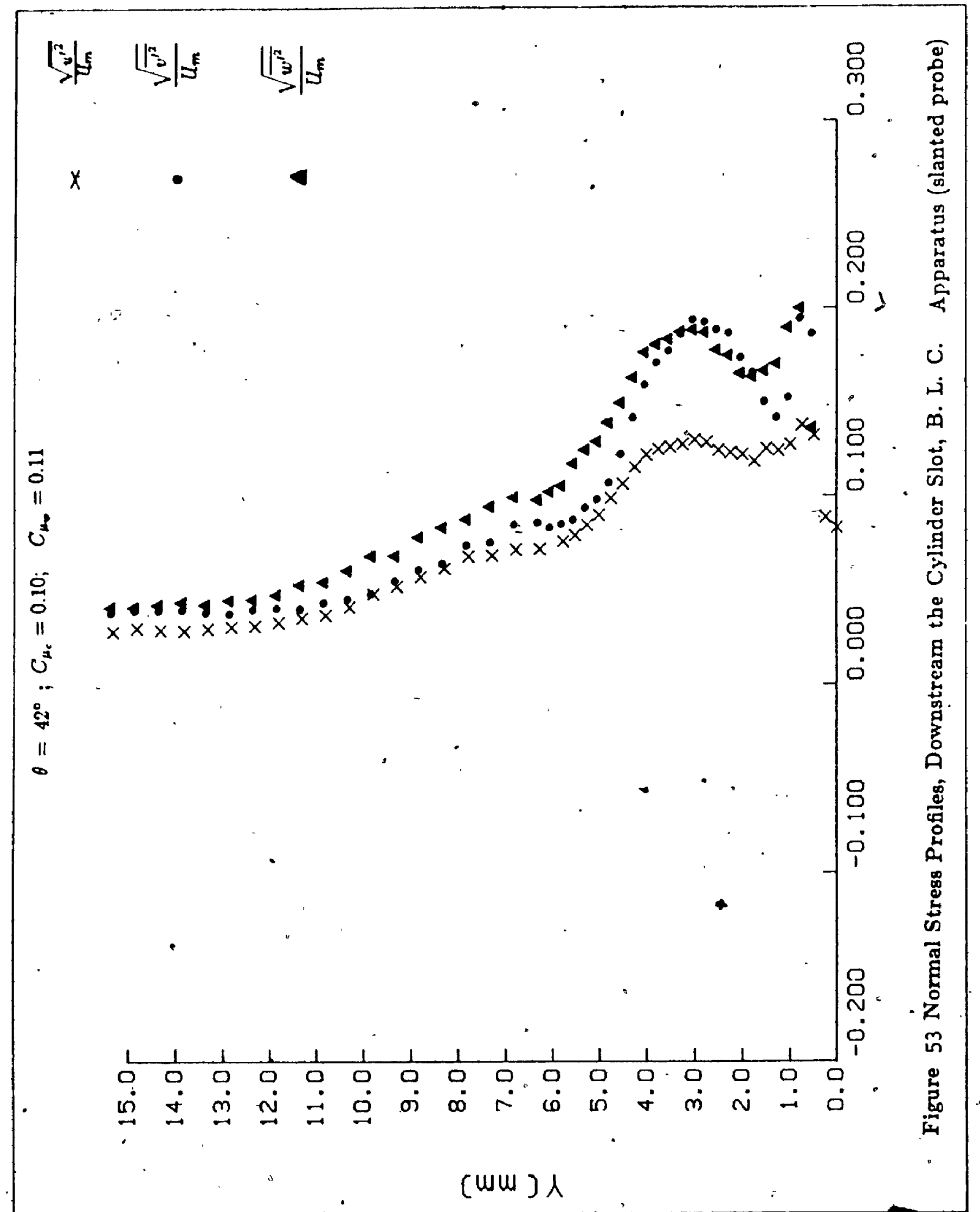


sis

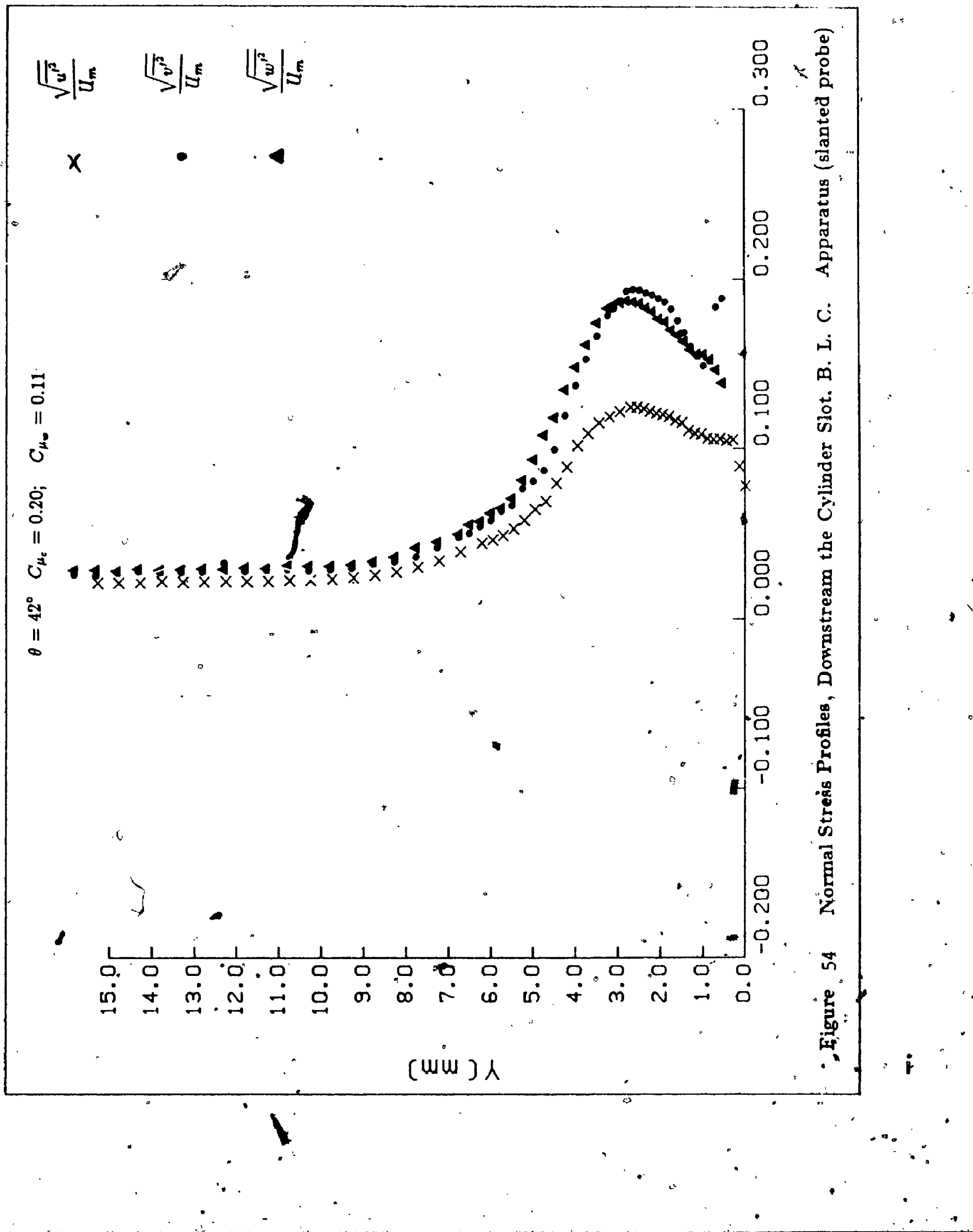




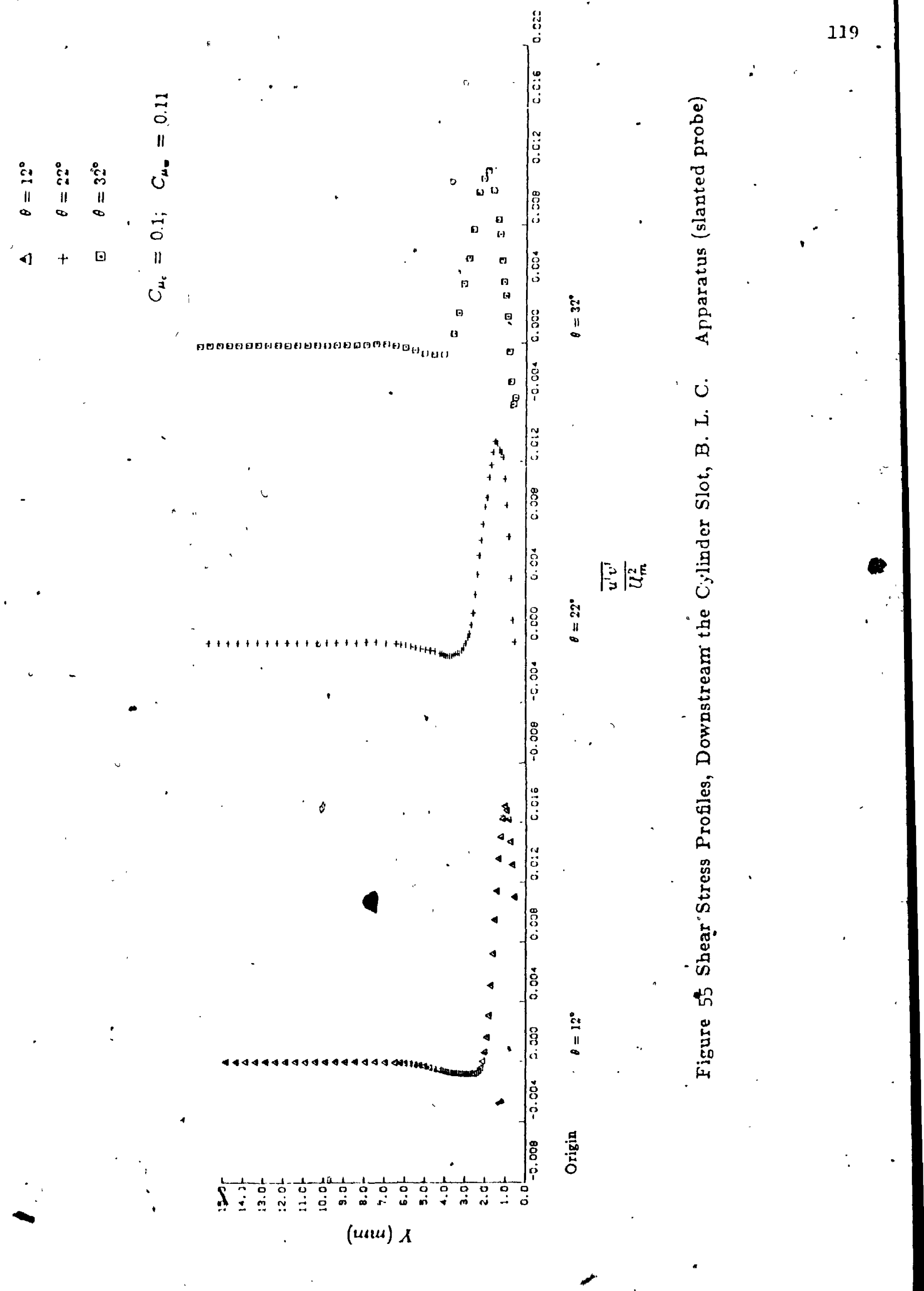




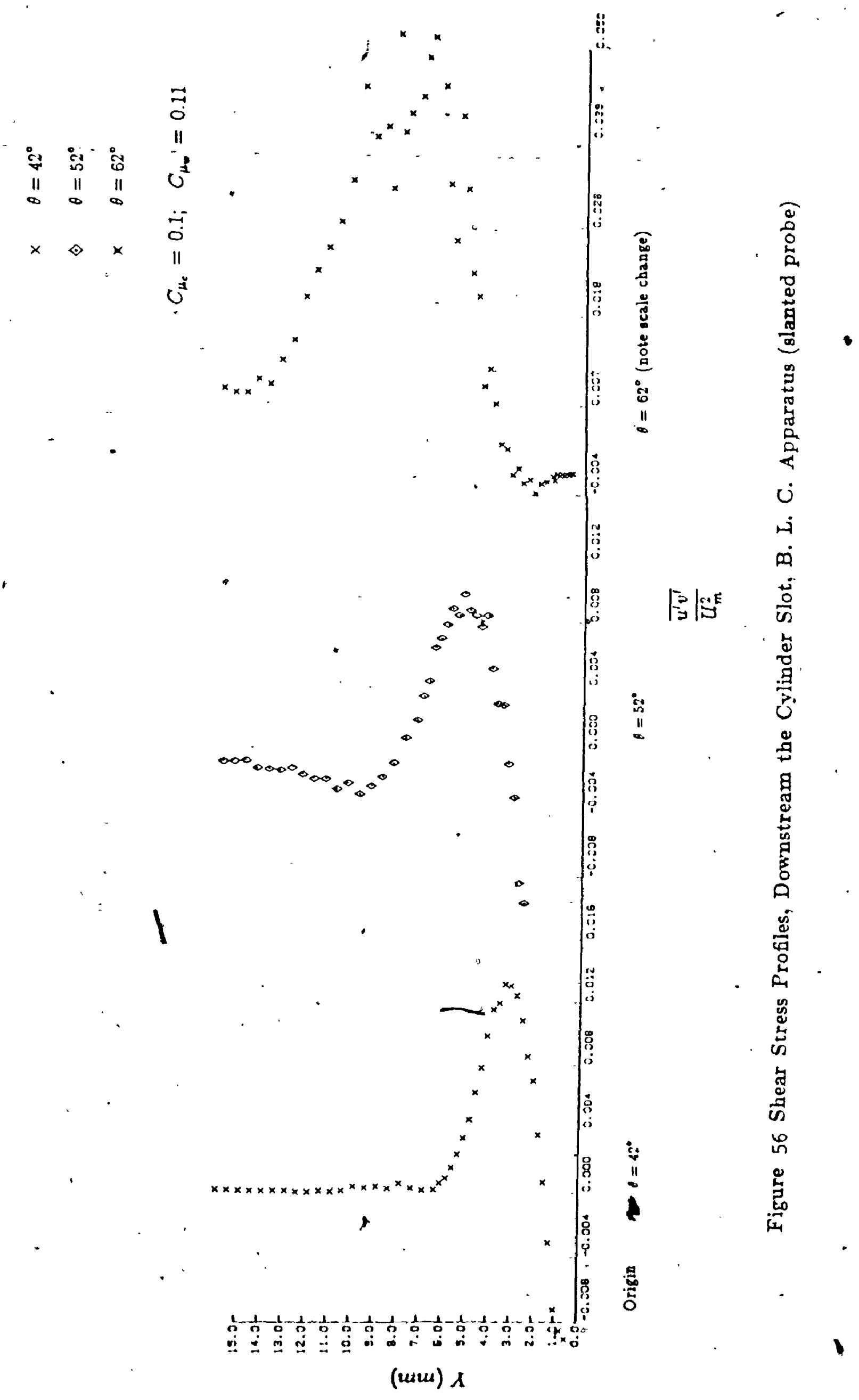




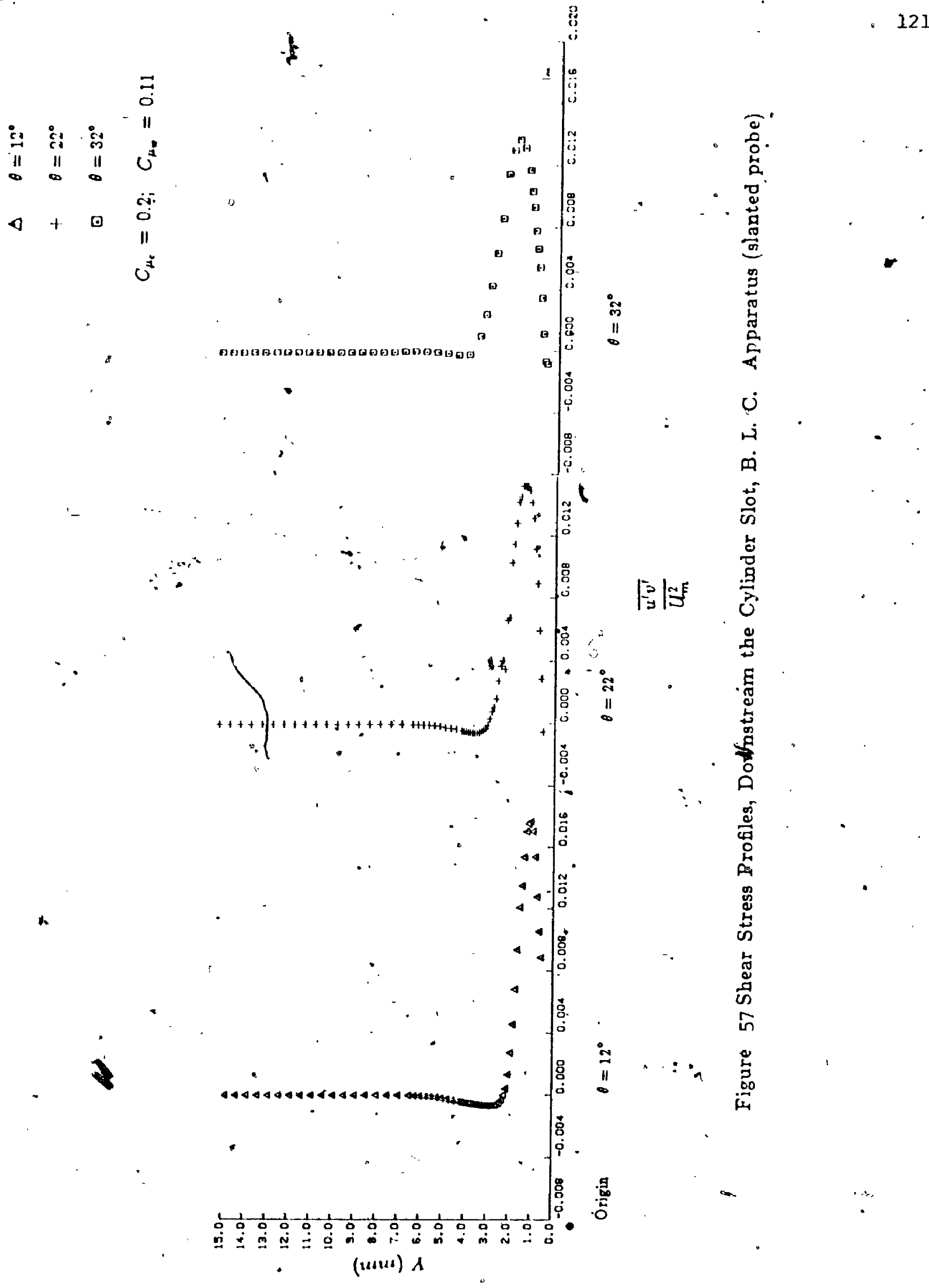




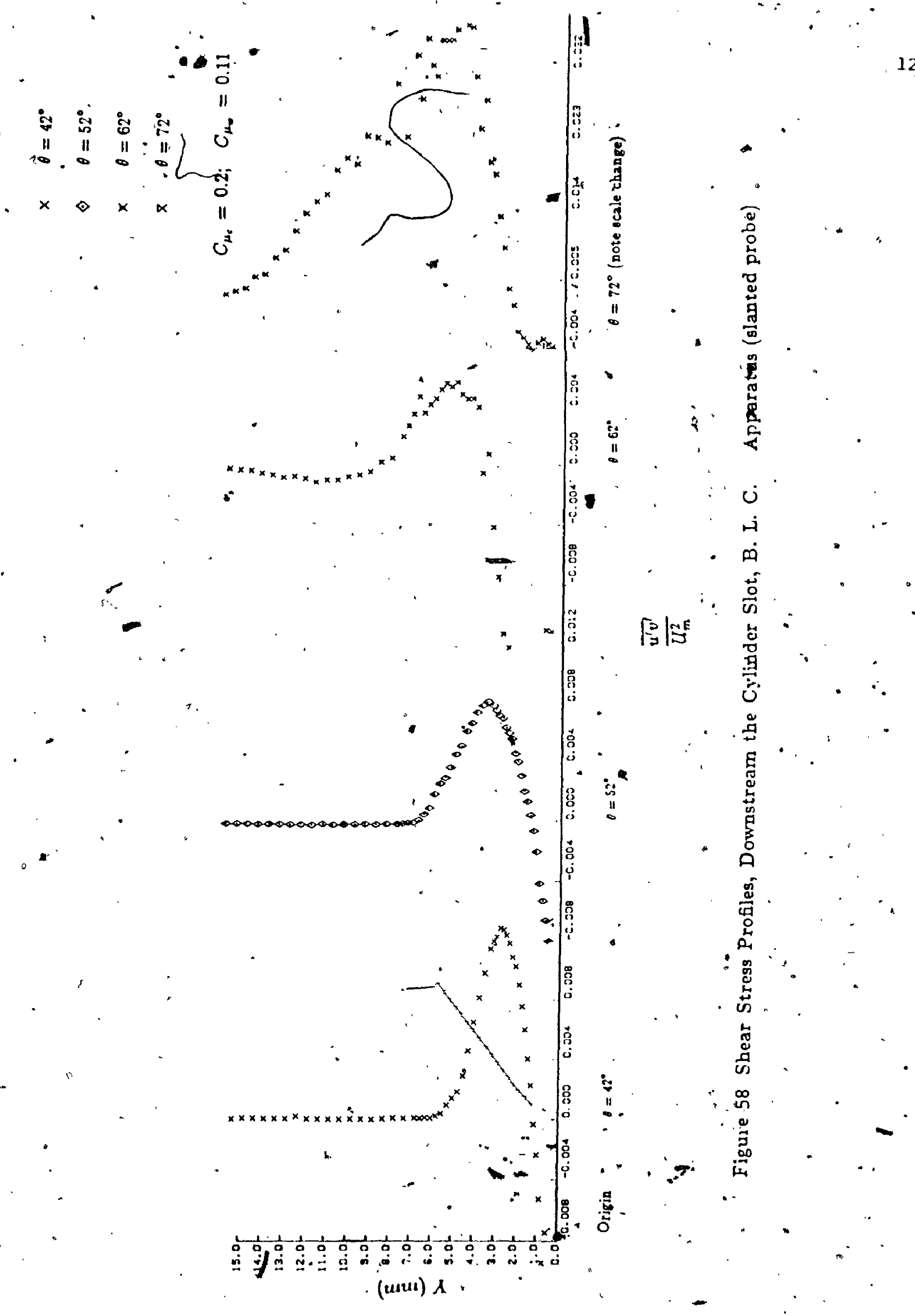




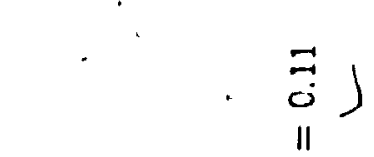

\begin{tabular}{ccc}
0 & 0 & 0 \\
\hdashline & 9 & 5 \\
11 & $\| 1$ & $\| 1$ \\
0 & 0 & 0
\end{tabular}

$1+0$

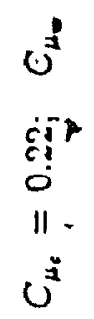

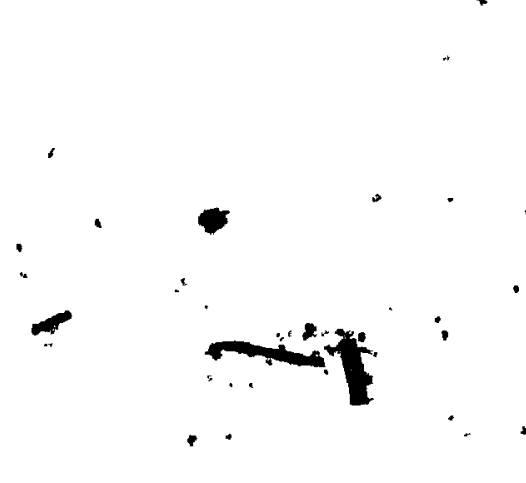

-<smiles>Ic1ccccc1</smiles>
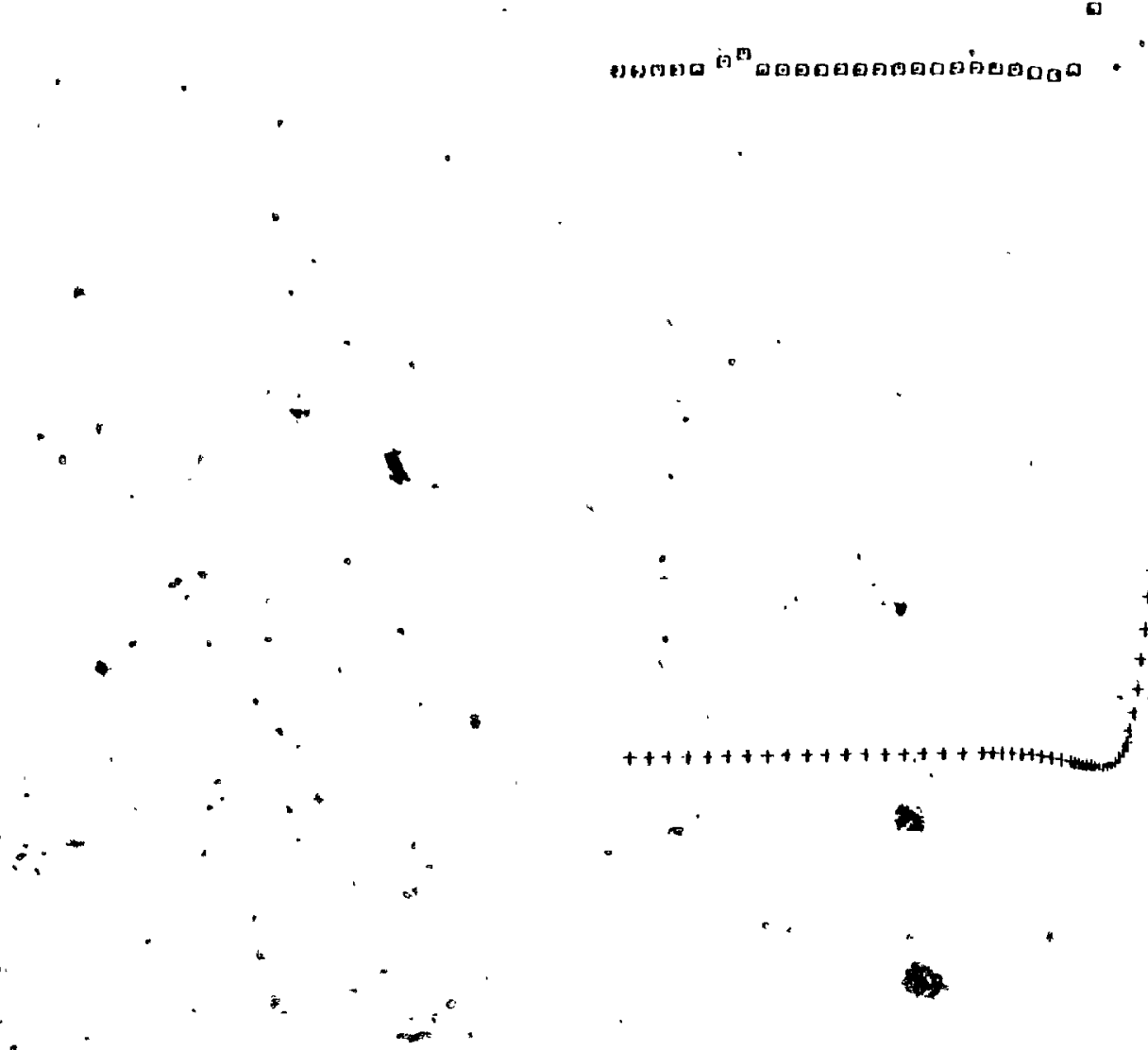

$\lambda$
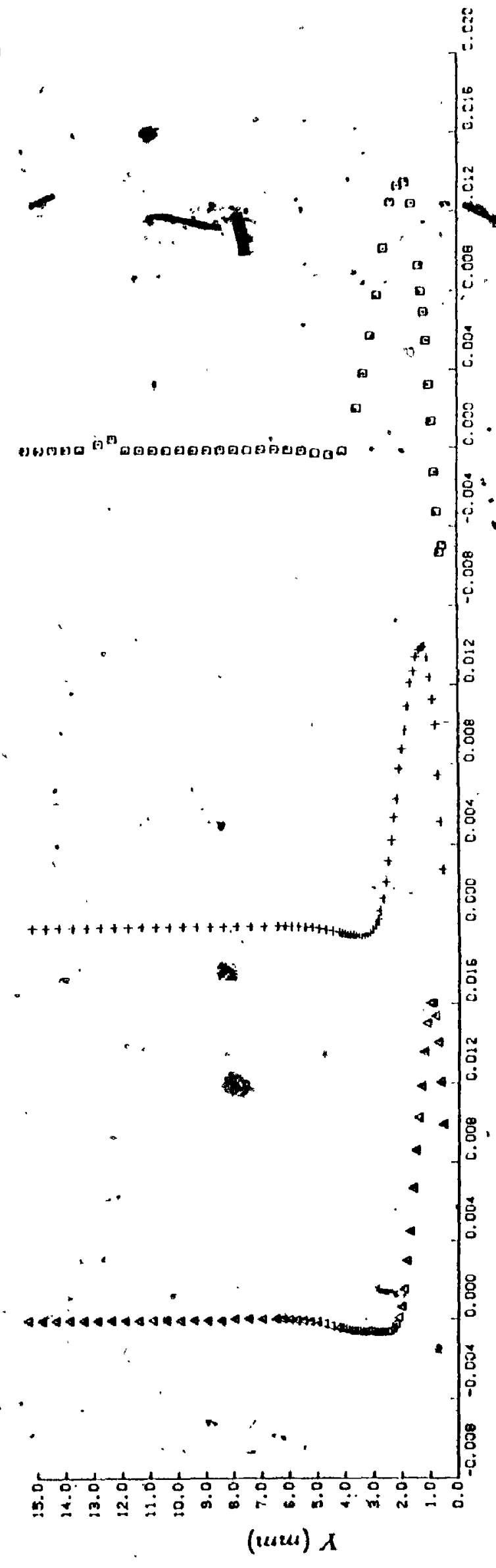


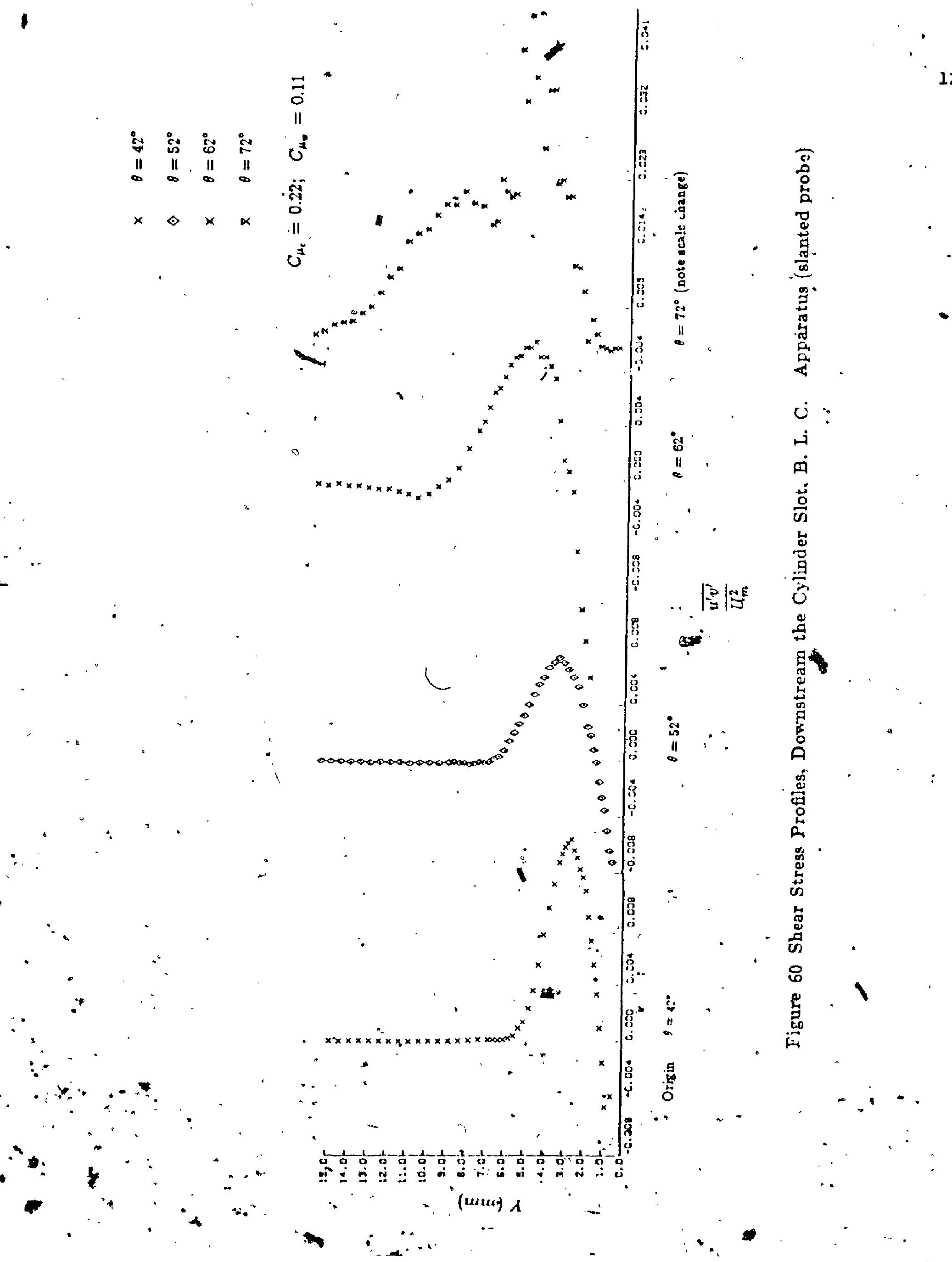



Fily

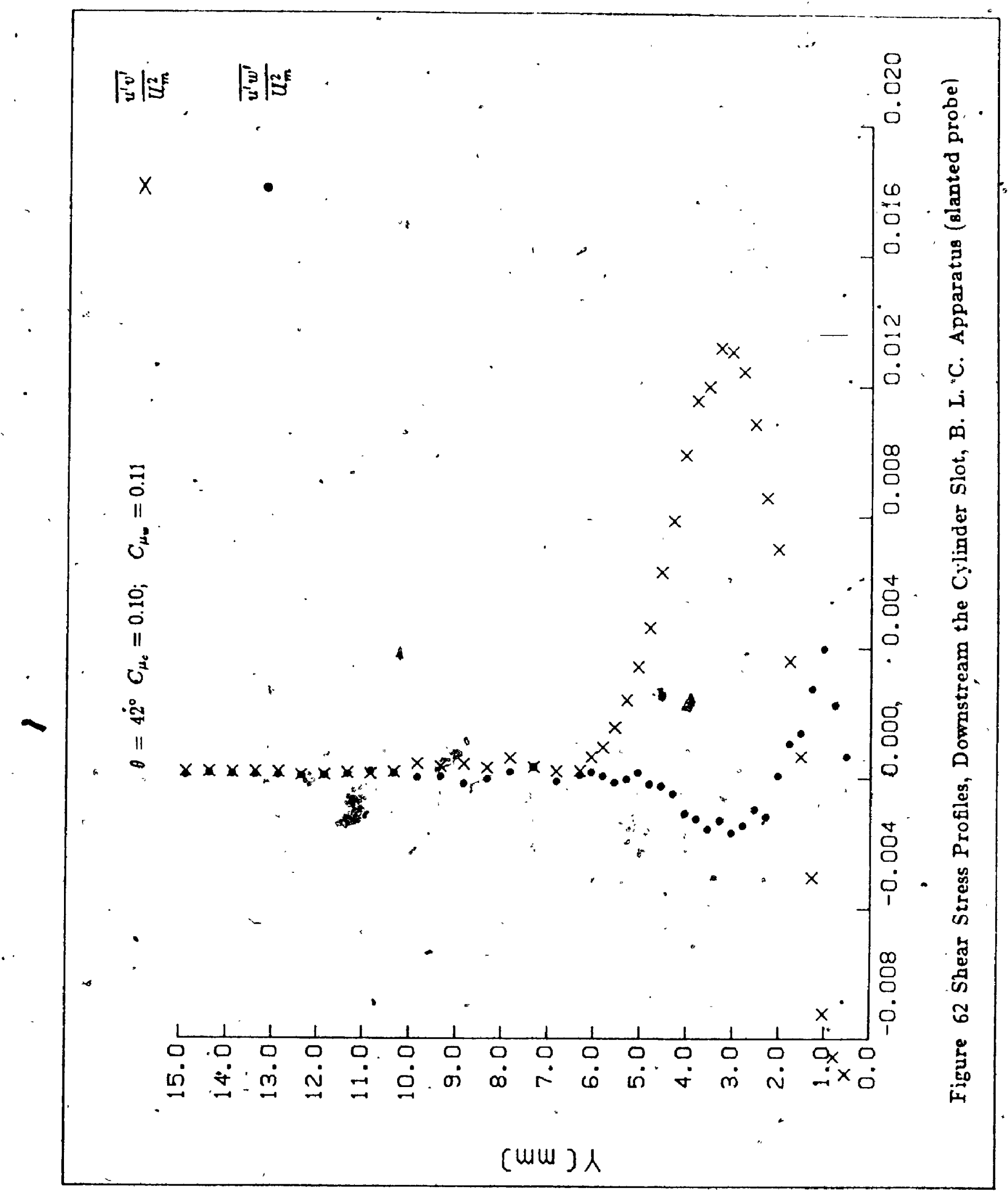




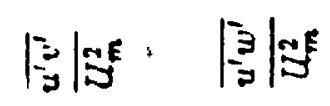

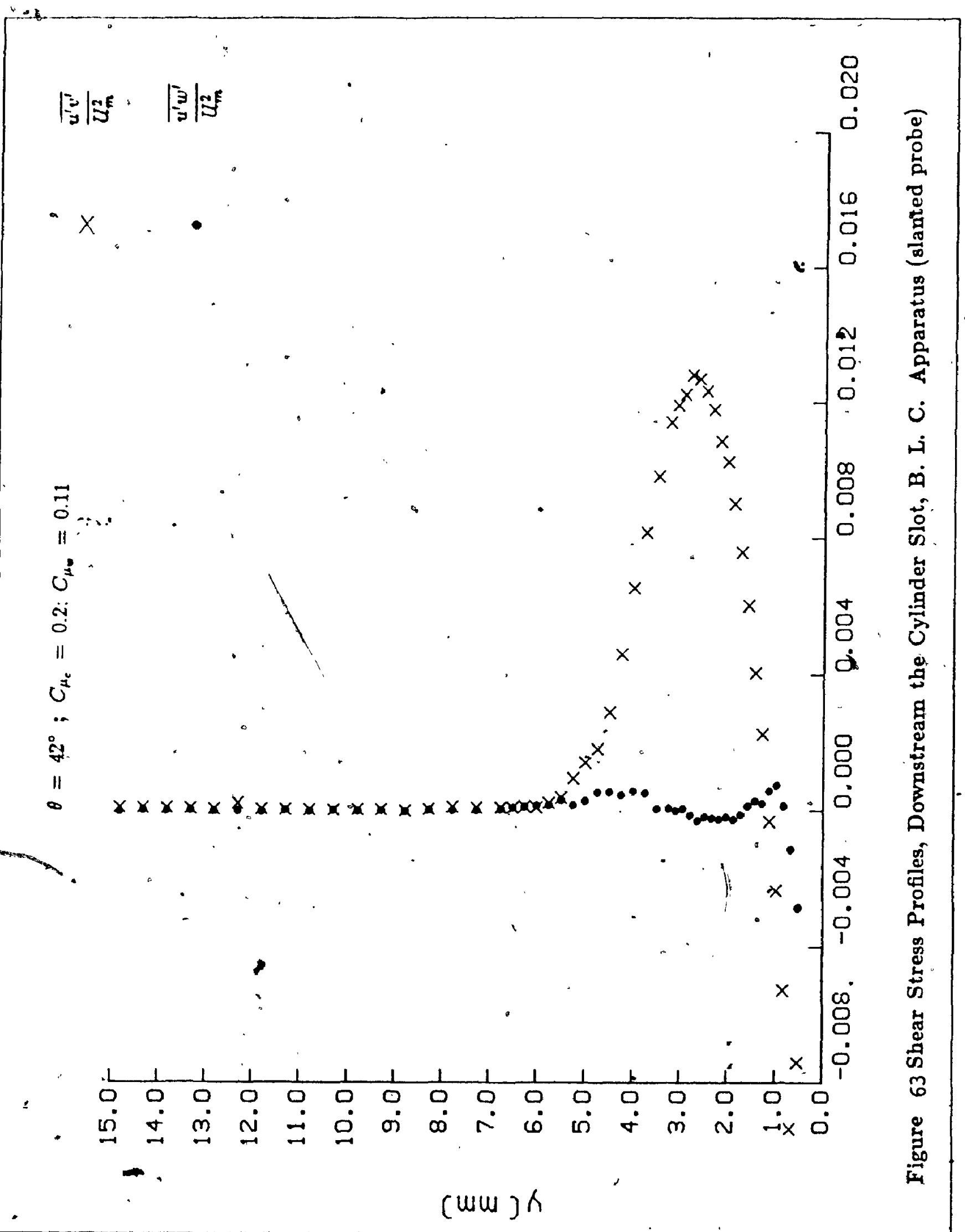




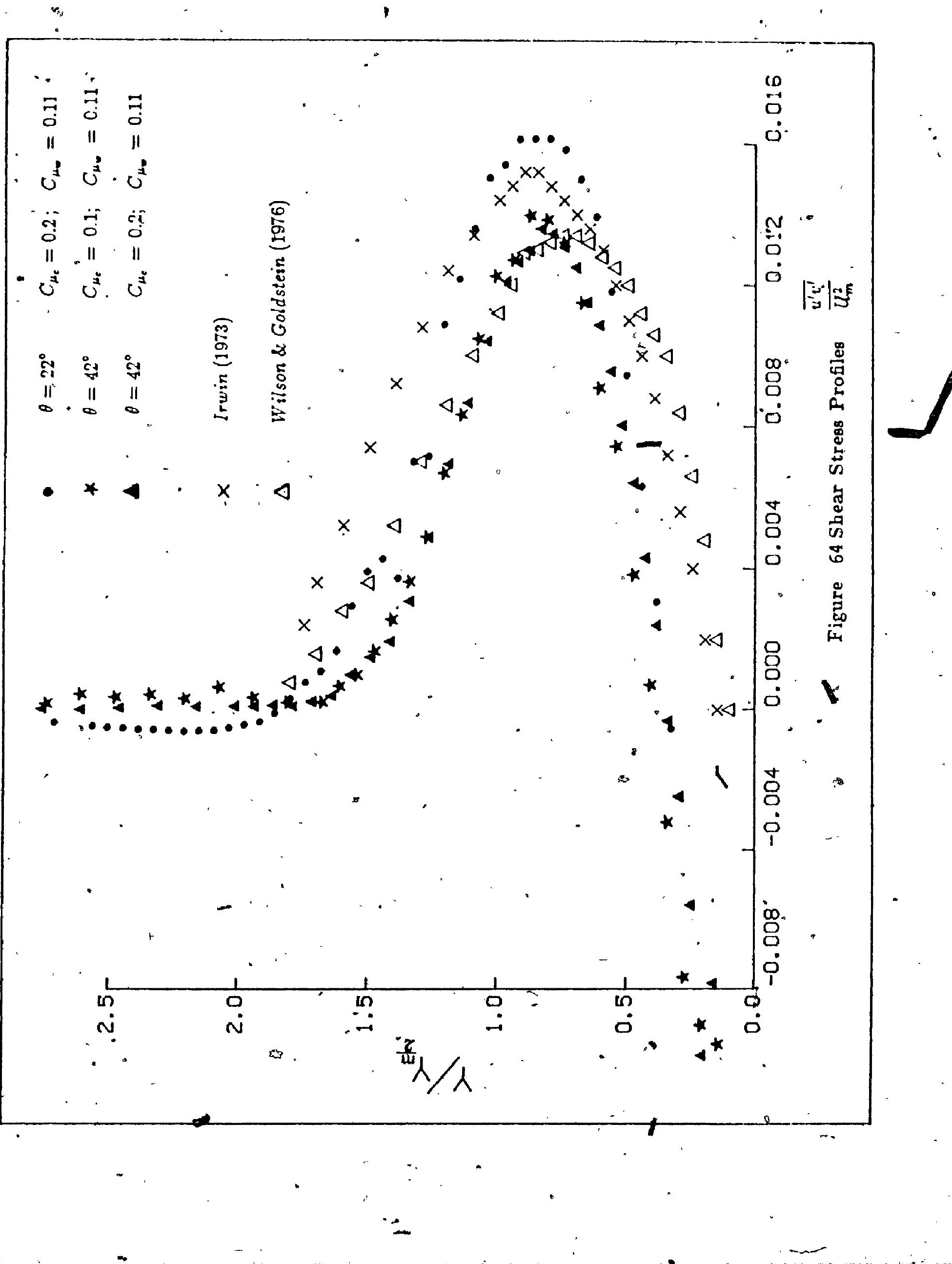




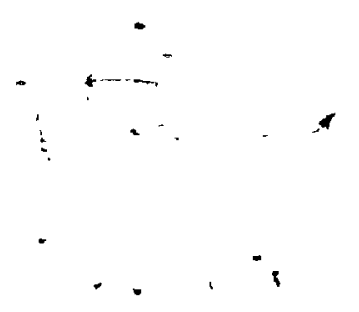




\section{A pipendix A}

\section{Traversing Gear_Design.}

\section{A.1 Traversing Gear Kinematic Analysis}

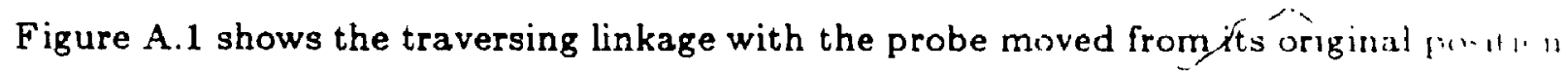
' $G$, to D. Point $W$ is the point that the pinion gear and guide tube connect when the pri 1 . is at zero (i.e. G) position. Point $O$ is the point around which the support tube $(1$, , pivots. Point $A$ is the point at which the guide tube is pinned to the support tube (1)ii) Distances $O W$, WA and $O G$ have to be measured before traversing. Thus, if 'attv' 1 ' : $: 11$ ' advancement of the guide tube during a traversing step;

$$
W N=W A+a d v
$$

From triangle $\mathrm{AOW}$, by the cosine rule;

$$
\begin{aligned}
W A^{2} & =O A^{2}+O W^{2}-2 O A O W \cos \phi \\
\phi & =\cos ^{-1}\left(\frac{O A^{2}+O \dot{W}^{2}-W A^{2}}{2 O A O W}\right)
\end{aligned}
$$

Applying the sine rule;

$$
\sigma=\sin ^{-1}\left(\frac{O A}{W A} \sin \phi\right)
$$

From triangle ONW,

by the cosine rule;

$$
O N^{2}=O W^{2}+W N^{2}-2 O W W N \cos \varepsilon
$$

by the sine rule

$$
\gamma=\sin ^{-1}\left(\frac{W N}{O N} \sin \varepsilon\right)
$$

and

$$
\delta=180-\gamma-\varepsilon
$$

and

$r$

$$
A N=W N-W A
$$


and from $A^{\prime} O N$ triangle

$$
\zeta=\sin ^{-1}\left(\frac{A N}{O A} \sin \delta\right)
$$

$$
\psi=90-\delta
$$

From triangle $O A^{\prime} N$

$$
k=\sin ^{-1}\left(\frac{O N}{O A^{\prime}} \sin \psi^{\prime}\right)
$$

and

$$
O A=O A^{\prime}
$$

and

$$
\begin{aligned}
\lambda & =180-\kappa-\psi \\
-\mu & =\lambda+5
\end{aligned}
$$

then from from triangle DOG, applying the cosine rulei

$$
G D^{2}=O D^{2}+O G^{2}-2 \dot{O} G O D \cos \mu
$$

and

$$
O G=O D
$$

then $\quad$ :

$$
\text { f } \quad G D=\sqrt{2 O G^{2}-2 O G^{2} \cos \mu}
$$

Therefore, by knowing the advancement of the guide at any instant we can find the actual ... - distance-of probe, (GD).

If the probe is traversed backwards the analysis is the same but $W_{N}=W_{A}-a d v ; \psi=$ $90+\delta$.

\section{A.2 Traversing Gear Error Analysis}

To determine the accuracy of the determination of the distance from the cylinder surface, $Y$, calculated following the analysis above, the results were compared to results from a graphical solution and all distances and angles were checked.

"Having ensured that the analysis gave correct results, the error analysis was performed. Thus, the measured distances $O W, W A$ and $O G$ were intensionaly, changed to determine 
the difference in $Y$ determined from the analyois. Thus, when $A W$ was changed $2 \%$ the distance $Y$ changed $0.5 \%(0.02 \mathrm{~mm})$, when $O W$ was changed by $1.5 \% Y$ changed $0.8 \%$ $(0.03 \mathrm{~mm})$, when $O A$ was changed by $1.5 \%$ the change in $Y$ was $1.8 \%(0.07 \mathrm{~mm})$ and an $O G$ change of $1.5 \%$ resulted in $Y$ changing by $1 \%(0.04 \mathrm{~mm})$.

Because the distances $O A, O G$ and $A W$ could be measured by a micrometer, the errors were minimized. The overall error in $Y$ is estimated to be less than $0.03 \mathrm{~mm}$. Effects of traversing pinion slip were minimized by using a 10:1 gear ratio as deacribed in Section. 2.2.6. Errors in $Y$ determination resulting from arc length, were minimized since $Y_{\max }$ was $1.5 \mathrm{~mm}$ and the support tube length, $O G$, was kept as long as possible, typically $40 \mathrm{~cm}$.

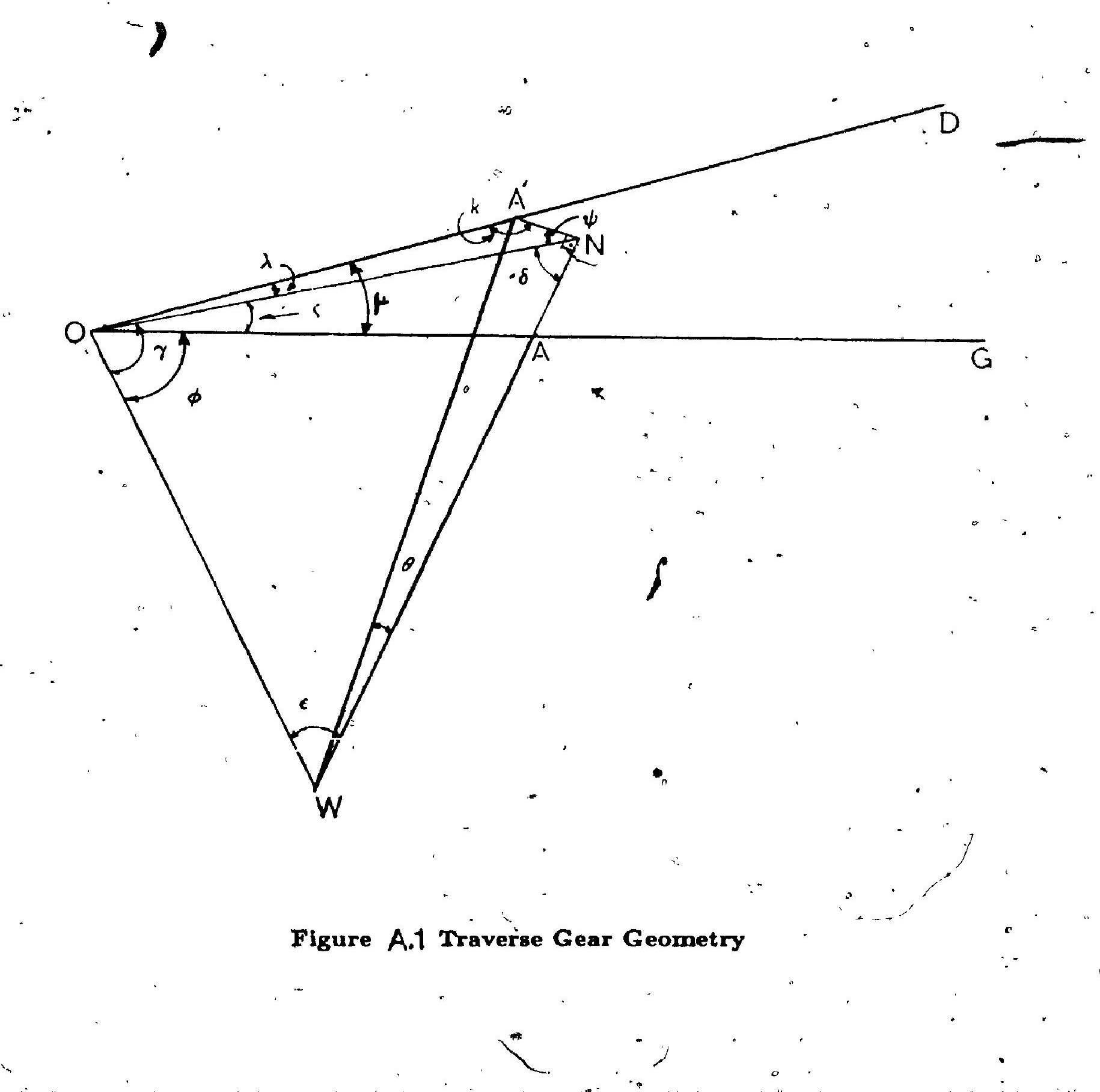




\section{- Appendix B \\ Compute Programs for Calibration of Hot-Wire Probes}

- B.1 Computer Program "CALIB*"

A brief description for the main parameters of the program and its statements are given below along with the flowechartand the program listing.

$\mathrm{CO}($ ) Coefficients uged for linearization of bridge mean and RMS signals.

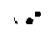

C1 ( )

Calculated linearized mean bridge voltage output, $\bar{E}_{\text {lin }}$.

E1 ( ) Percent érror in linearization plot.

Fo ( ) Average transducer output voltage.

F1 ( ) Flow velocity calculated in $\mathrm{ms}^{-1}$.

I0 ( ) Slope of transducer calibration curve; (i.e. $10=0.1626$ ).

KO ( ) Transducer voltage output, read by the HP 3456A voltmeter.

L1 (ג) Coefficient to be used in setting TSI electronic linearizer unit.

No Number of data pairs to be read; input $\equiv 20$.

N8 Normalized flow velocities.

$\mathrm{PO}()^{*}$ Average mean bridge output voltage, $\bar{E}_{b r}$.

S6

TO ( )

Slope of $E_{b r}$ vs $U$ curve.

Bridge mean output voltage, redd by the HP $3456 \mathrm{~A}$ voltmeter.

8
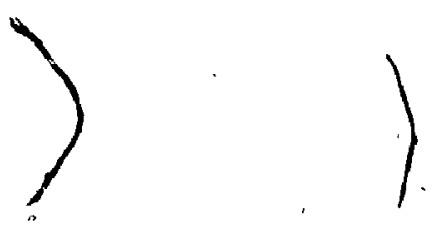
YO () Flow temperature; in put degrees Celsius.

Program Description

line 4 Description

$10-160$ Program title, function and date of experiment.

$170-240$ Deceleration variables and dimensioning of arrays.

$320=370$ Input number of data sets to be read and the slope of transducer calibration curve.

490-510 Initialization of ADACS system and set HP 3456A voltmeter to take readings.

$520-590 \quad$ Acquisition and averaging of mean bridge output signals, using ChanneF 1.

$600-790$ Acquisition and averaging of pressure transducer output voltage and calculation of flow velocity in $\mathrm{ms}^{-1}$.

800-820 Print $\bar{E}_{b r}$, flow velocity (ms $y$ and corresponding temperature in ${ }^{\circ} \mathrm{C}$.

Press "continue" key tip procede with next set of readings.

$910-1090^{\circ} \quad \bar{E}_{b r}$ v8 $U^{\prime}\left(m^{-1}\right)$ plotting routine.

$1160-2360$ Bödy of linearization routine, converted to BASIC from FORTRAN yersion of TSI manual.

2370

Print of coefficients to be used in latter linearization of mean and RMS bridge signals.

$a$

Print of coefficients for setting linearizer coefficients in TSI linearizer unit. 
3140

$\mathbf{3 2 3 0}-\mathbf{3 5 9 0}$

$3620-4540$

$4550-4800$

Print the slope of linearizer curve, $\bar{E}_{\text {lin }}$ vB $U$.

Plotting of $E_{\text {iin }}$ vo $U$.

Subroutine "init", to initialize ADACS system.

Subroutine "AVDCV", to average DC voltage from bridge output. 


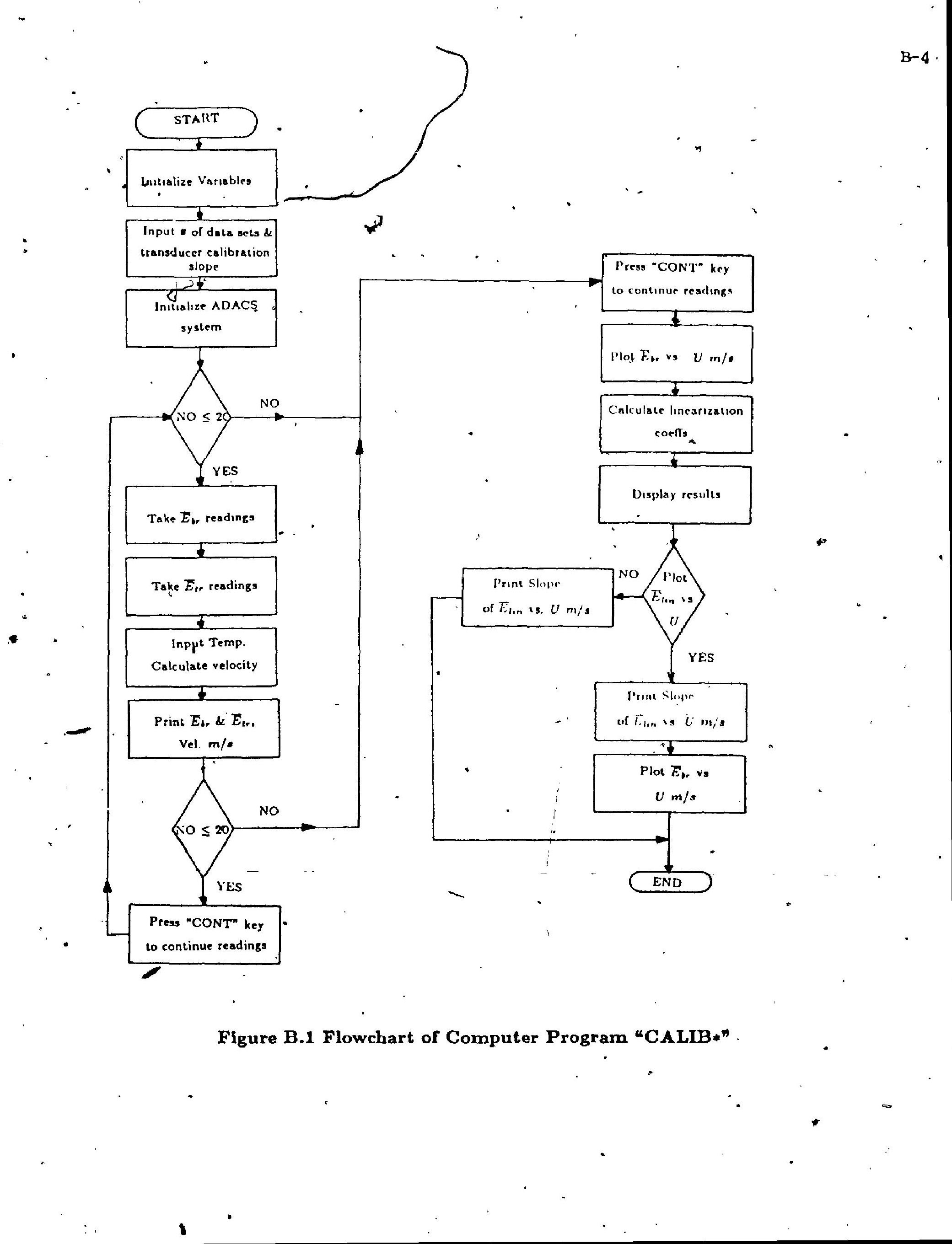




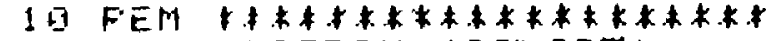

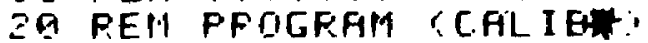

3D REM $* * * * * * * * * * * * * * * * * * * *$

4t FEH This program determines

59 REM the LIMEARIZER coet $f$ 's

BG REM tot HOT-WIRE ariemometer

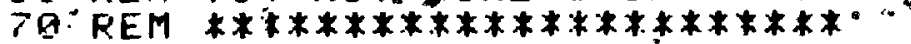

EIQ I

Qil DISP' "IHPUT DATE"

Q INFUT [IS

83 PFINT."

\$4 PFINT "CALIBFATION APR-":

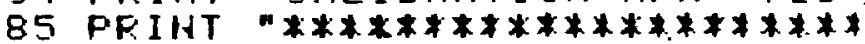

"P

BE PFIIHT

8. PRINT;

जe !

199 OPTIOH BASE \$ 1101$), C 0(8), E 1($

110 REFL Ar.8, 8 ), C1:2

129 REAL NE(21),PO(21)

139 REAL R( 21$), S(9), W(21), \times 5(8, g$ D. UG(21), T(21), FQ(21)

140 DIM XIर21), $8 \bar{r}(21), 70(39)$

159 REAL U?21).0(21). RQ(30)

15 G REL X $(21)$, WO 21$)$

$17 \mathrm{G}$ !

139 REM ***********************

19 G REM F1 = NELDCIT $r(m) s)$

209 REM $P G=B R I D G E$ VOLTAGE (volt)

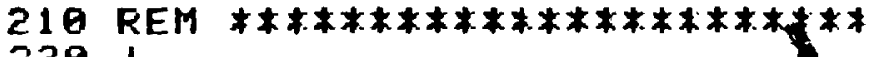

\section{-}

23

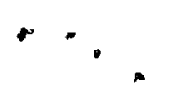

2490

250 DISP "Enter number of DATA P

200 INPUT $H B$

270 DISP "Enter slope of TRANSDU

CER calib. i i.e $=1025)^{\prime \prime}$

28日 INPUT IB

29日!

$390 \cdot$

319 PRINT LISING 320

320 IMAGE "VEL (m,s)",5X, "B. VOLT" IMAGE " $5 \times$ "TEMP(C)"

- 336 PFINT USING 340

349 IMAGE,

350 !

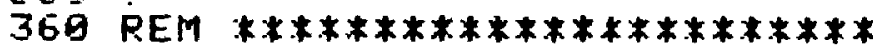

370 REM Main program (calib.)

389 REM USing DATA-ACQUISITION

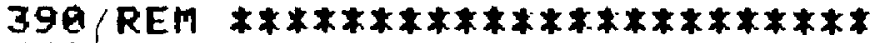

409 !

410 !

420 PI $=3456 !$ DIGITAL WOLTMETER.

$430 !$

440 G0SUB 3390 ' Subroutine "ini

45 FOR I $=1$ TO NO

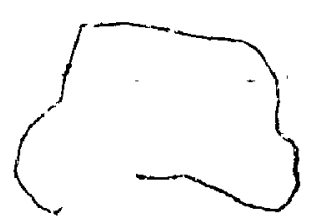


千こ !

$469 \quad P 1=1$

$479 \quad 5 a=8$

480 FOR I $1=1$ TO 20

490 GOSUB 4310 ! Subrt" "Fde."

$5 B \theta$ TO(I1)=P e $5 \theta=\$ \theta+T \theta(11)$ e HE

ins

$519 P_{1=2}$ ! CHANINEL ;

$511 Q 9=0$

512. FOR $M=1$ TO NÖ

513 !

$514 !$

529 Gosub-4310! Subrt "Avdcr"

- 521 Ka $1 M J=P$

$522 \mathrm{QB}=\mathrm{QB}+\mathrm{KB}(\mathrm{M})$

523 NEST $M$

- $524 F G(I)=12 B<29$

$539 F G(I)=F$

$549 \mathrm{FQ}(1)=F \theta(1) *(\vdots 1)$

$550 \quad Y=F Q(1)$

$56 \theta$ WE $(I)=F B(I)-Y$

501 IISP "InPU T TEMPERATURE (C)"

562 INPUT YO

57日 WG( 1$)=W 0(I) \neq 1000$

- $57101=353.266953 /(273.1+Y 9)$

$5 \% 2$ FI(I)=4:02486173875QR (HO(I) IB. 01$)$

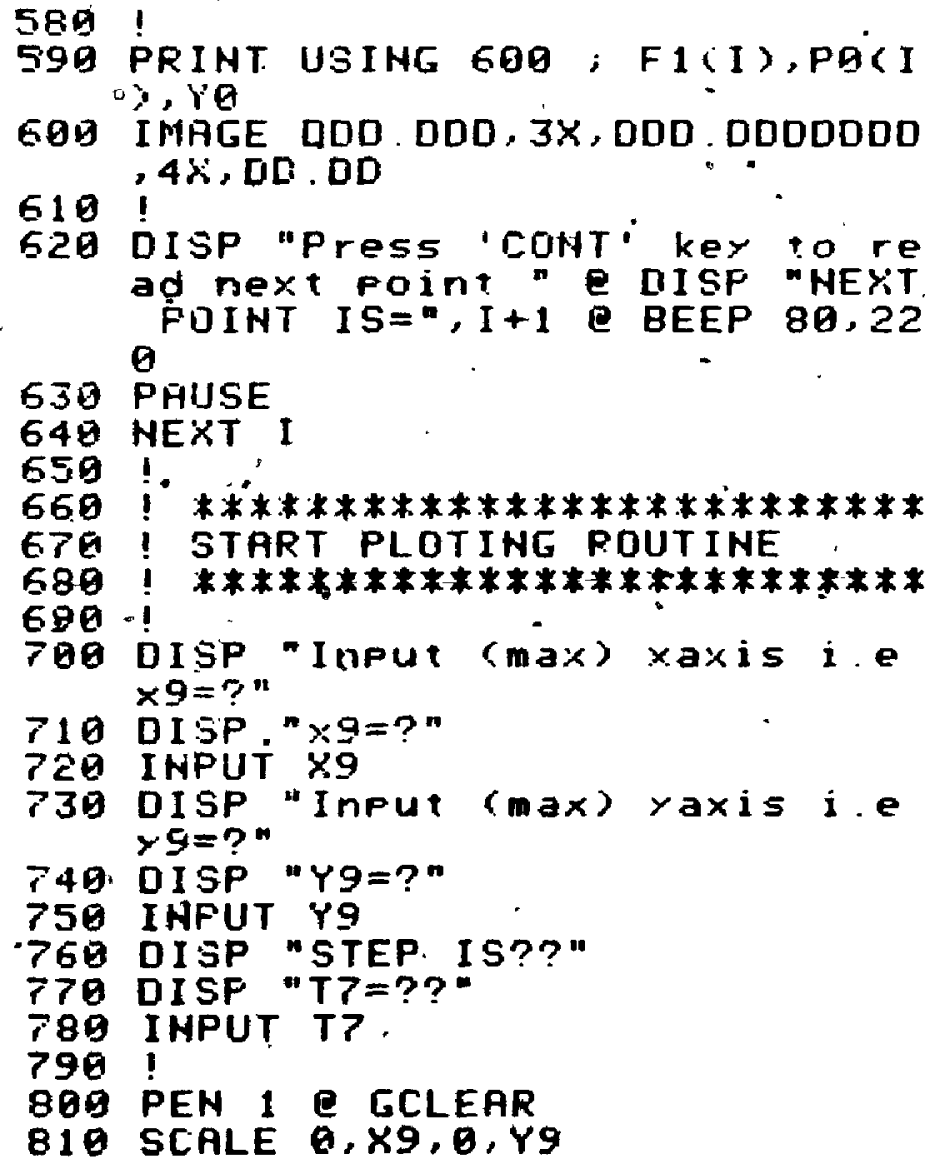




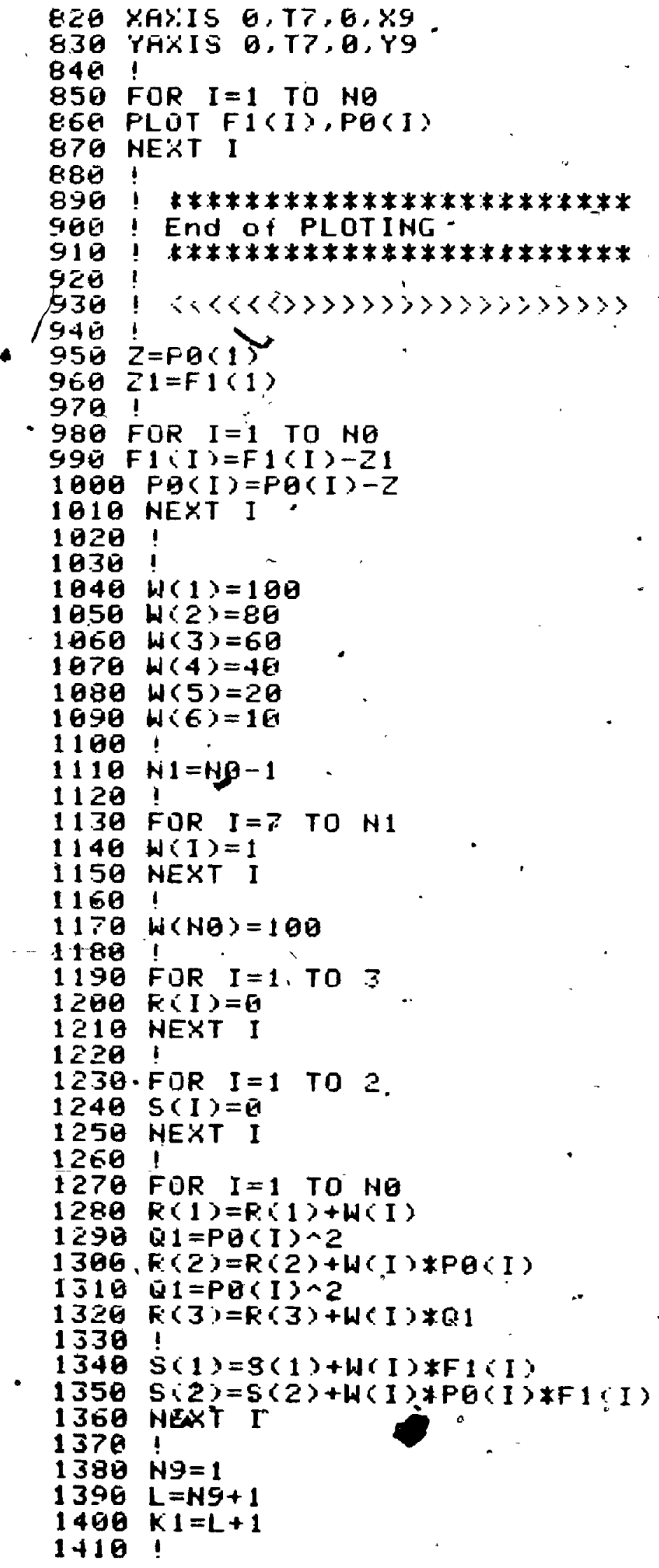


1420 FOF I $=1$ TO L.

1430 FUR $J=1$ TOL.

1440. I $2=\mathrm{J}-1+\mathrm{I}$

$1450 A(I, J)=R(12)$

1469 NEXT I

1470 !

$1480 \mathrm{~A}(\mathrm{I}, \mathrm{K} 1)=S(I)$

1490 NEXT I

1509

1510 FOR $I=1$ TO.L

$1528 f(k, 1, I)=-1$

$1530 \mathrm{~K} 2=\mathrm{I}+1$

1540

1550

1569

1579

1580

1590

?.

. 1

1609

1619

$1629 \mathrm{FDR} \cdot \mathrm{I} 1=2$ TO $\mathrm{KI}$

1630 FOR $J=K 2$ TO $K 1$

$1640 A(I 1, J)=A(I 1, J)-A(I 1, I) \star A(1$

$\left.1850^{\circ}, J\right) \neq C$

$F D R \quad J=K 2$ TO $K 1$

$A(K 1,1)=0$

NEXT J

$! \div$

$C=1 / A(1, I)^{\prime}$

1660 HEXT I 1

1670

1689

1696

1709 FOR $J=K 2$ TO $K 1$

i.

$F O R \quad I 1=1$ TO $L$

1710 A $(I 1, J)=A(I 1+1, J$;

1720 NEXT I

$\therefore 1730$ NEXT I 1

1740 NEXT 1 .

$1750 !$

1760

1779 FIR I=1 TO L

1780

1799

$x 5(1, K 1-Z)=A(1, K 1)$

1819

- 1820

NEXT I

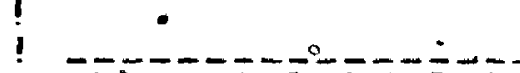

1830

1640

1850

1860

$18>0$

1889

1890

1990

1.910

1926

1930

1940

1950

1960

1970

IF

!

$!$

$\mathrm{NG}=\mathrm{H9}+1$

$j=2 * N 9$

$R(J)=0$

$R(1+1)=0$

$S(N 9+1)=9$

!

FOR: I-1 TO NO

Q2 $=P Q(1) \wedge(J-1)$

$R(J)=R(J)+Q 2 * w<. T$

Q3=PQ(i) N J

$R(J+1)=R(J+1)+Q 3 * H(I)$

Q4 =PQi(I) M 9 
$2010 S(N 9+1)=S(N 9+1)+F 1(I) * Q 4 * W($

1)

2029 NEST I

2630

2040

2050 GOTO 1390

2000 PRINT USING 2070

2061 PRINT

2065 PRINT USING 2070

2070 IHAGE "The 5 coefticients a

2671 PRINT

2 Q89 $M l=01+1$

2890 FOF $I=1$ TO $M I$

$2100 \cdot C \theta(I)=A(1, K 1)$

$2110 R=P \theta(N \theta) \wedge(I-1)$

$2120 \times 5(1,4)=\times 5(I, 4) * Q$

2130 PRINT CO(I)

2140 NEXT I

2150 !

2160

2170

2180 FOR $I=2$ TO 5

- $2190 . Q 5=P \theta(J 2) \sim(I-1)$

$22 \theta B(9=C 9+C B(1) * Q 5$

2210 MEXT I

2220

2239

$224 \theta^{\circ} \mathrm{C} 1(\mathrm{~J} 2)=\mathrm{C} 9 * 1 \mathrm{\theta}-\mathrm{F} 1\left(\mathrm{NG^{ \circ } )}\right.$

$2250^{\circ} E 1(J 2)=\left(C 9-F_{1}(J 2)\right) * 10 \theta / F_{1}(J$ 2)

$2260 \mathrm{H}$

$2270 !$

2280

2290

2390

2310

2320

2330

2340

2350

2360

2370

2380

2390

2400

2410

2420

2430

2440

2458

2460

2478

2489

2490

2590 NB(I) $=F 1$ ( I ) *56
2510 PRINT USING 2520

2520 IMAGE

2530

$S 6=10 / F 1(N O)$

FOR I =1 TO 4

$L I(I)=\times 5(I+1,4) * 56$

NEXT I

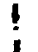

PRINT USING 2378

IMAGE

$!$

PRINT USING 2400

IMAGE "The 1 inearized coeff icients are:"

!

FOR I=1 TO 4

PRINT USING $2440 ; L 1$ (I)

I MAGE $3 \times, 000.0000$

NEXT I

!

!

FOR I $=2$ TO $\mathrm{NO}$

$M B(I)=F I(I) \star 56$

! 


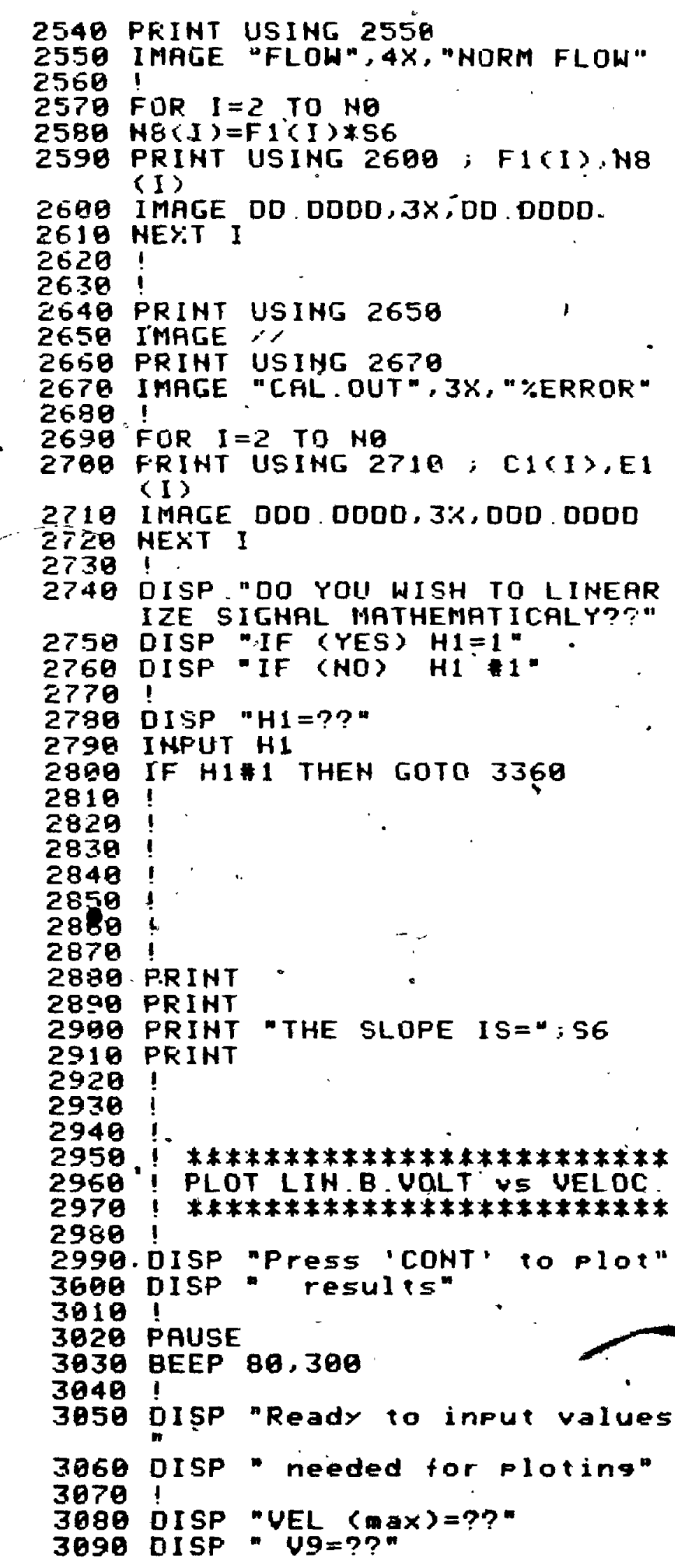




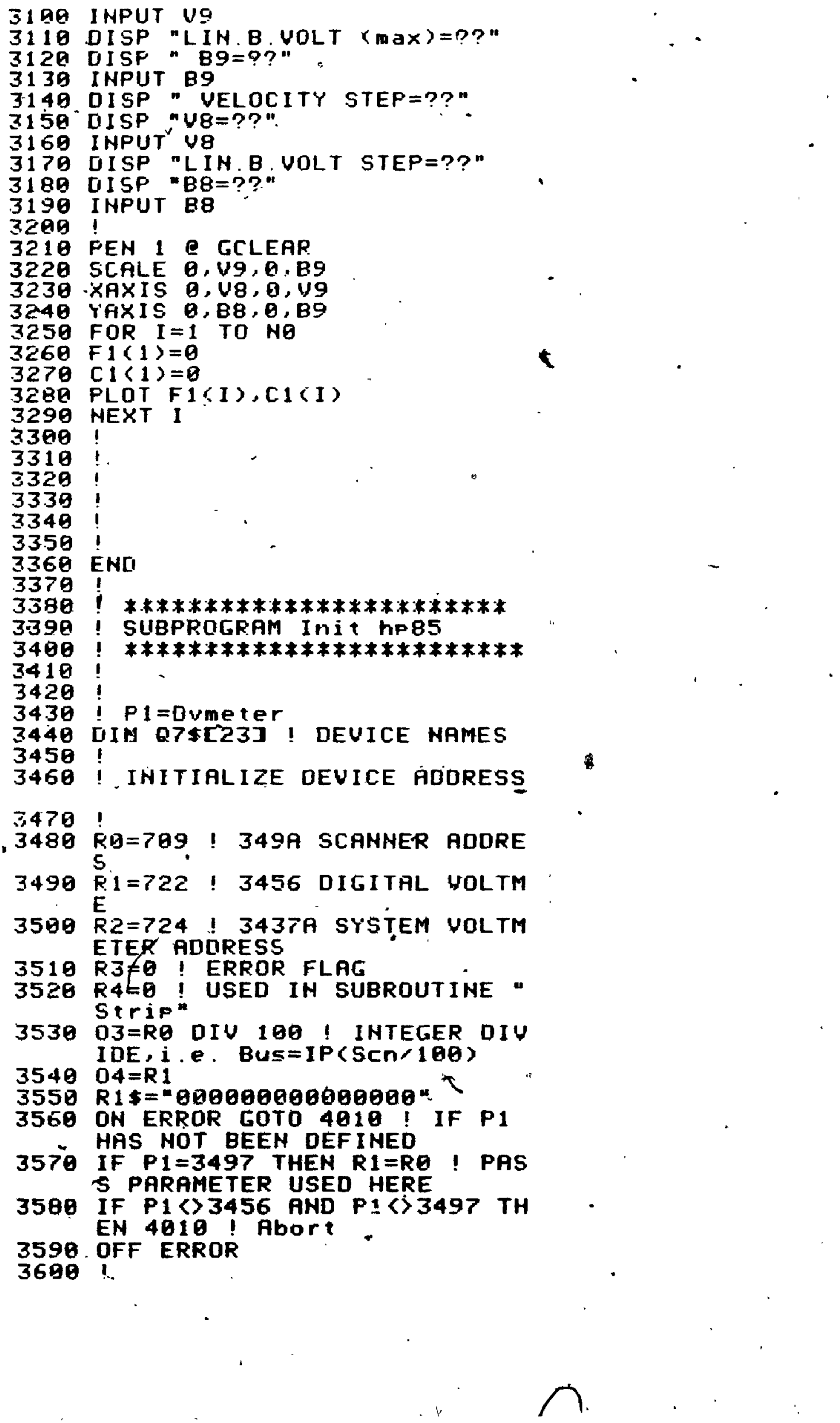




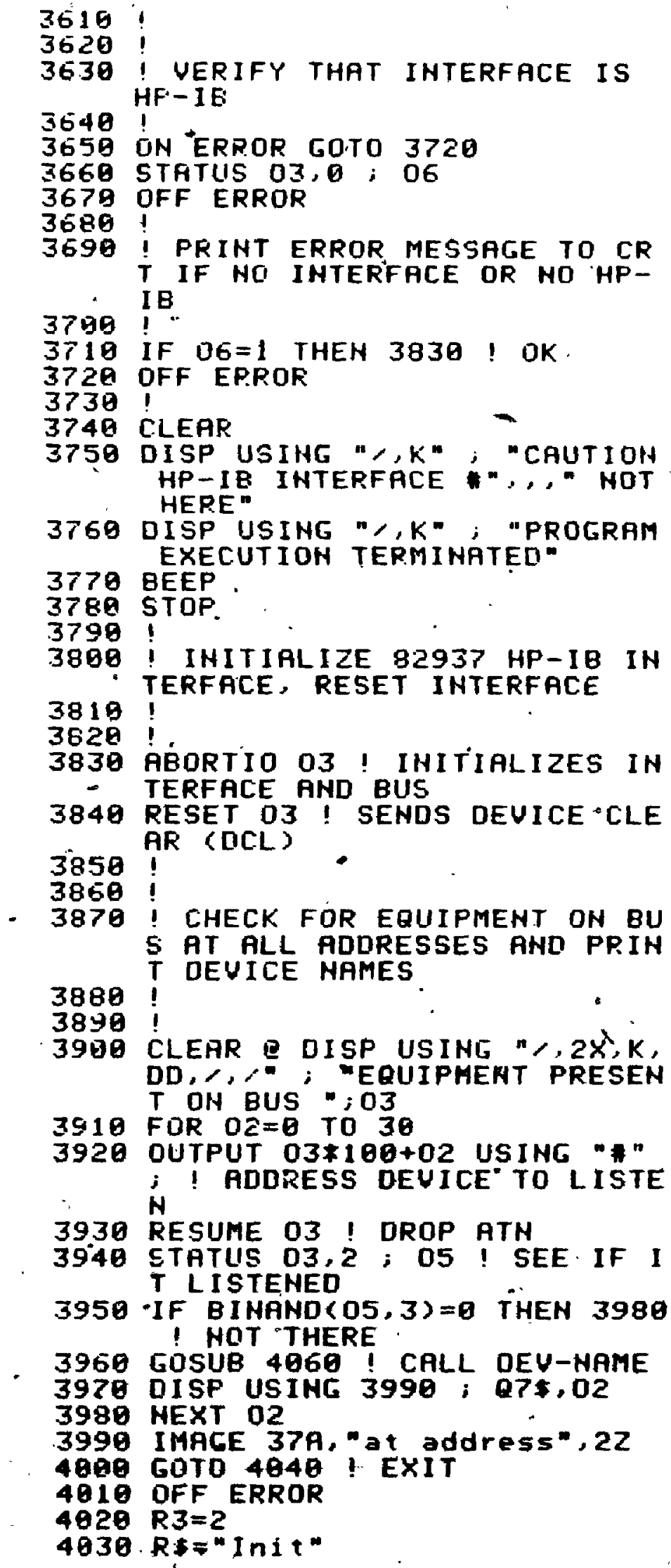




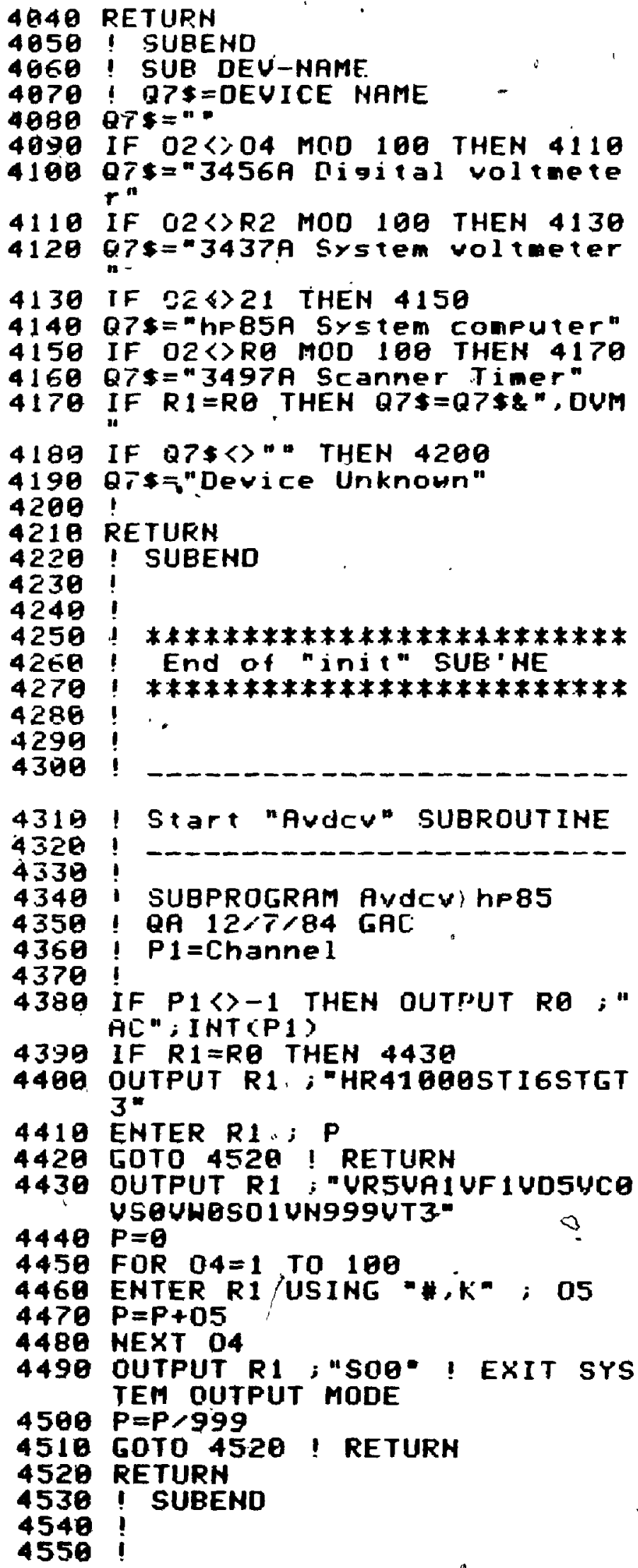




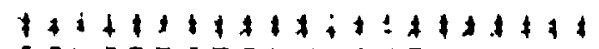

ERLIEFHTIBH HFE- 11

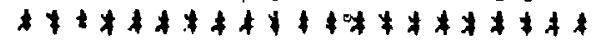

"EL' "I. $\equiv$,

E. WGLT

TEME: :-

5900

E1!

$\equiv$

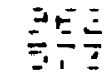

5 百

$\div \quad \overline{3}$

$\pm \frac{1}{4}=\frac{1}{4}$

$1 \sum_{10}^{-1}+\frac{1}{2}$

IE $3+5$

$50 \quad 3$

$=5$

34 irt

जT 4.

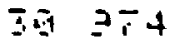

54,554

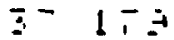

ID 45

4556

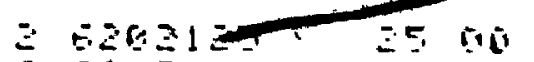

$=50$

$523+6$

5.50

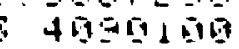

47198.19

56505

504545

7 105

$\Xi$ 是

$+640+5$

$4 \quad 2=14=5$

+ E17日2135

- 250245

$+2901515$

$4 \quad 5105$

4.4254685

4.506050

$+.55=250$

4. $590=5$

+. $535=30$

\begin{tabular}{ll}
50 \\
\hline 5
\end{tabular}

可

둥

$E 5 \quad 05$

E- 190

E

$\Xi 56$

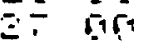

$\therefore \frac{1}{1}$

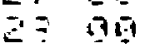

50

궁

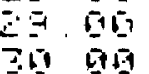
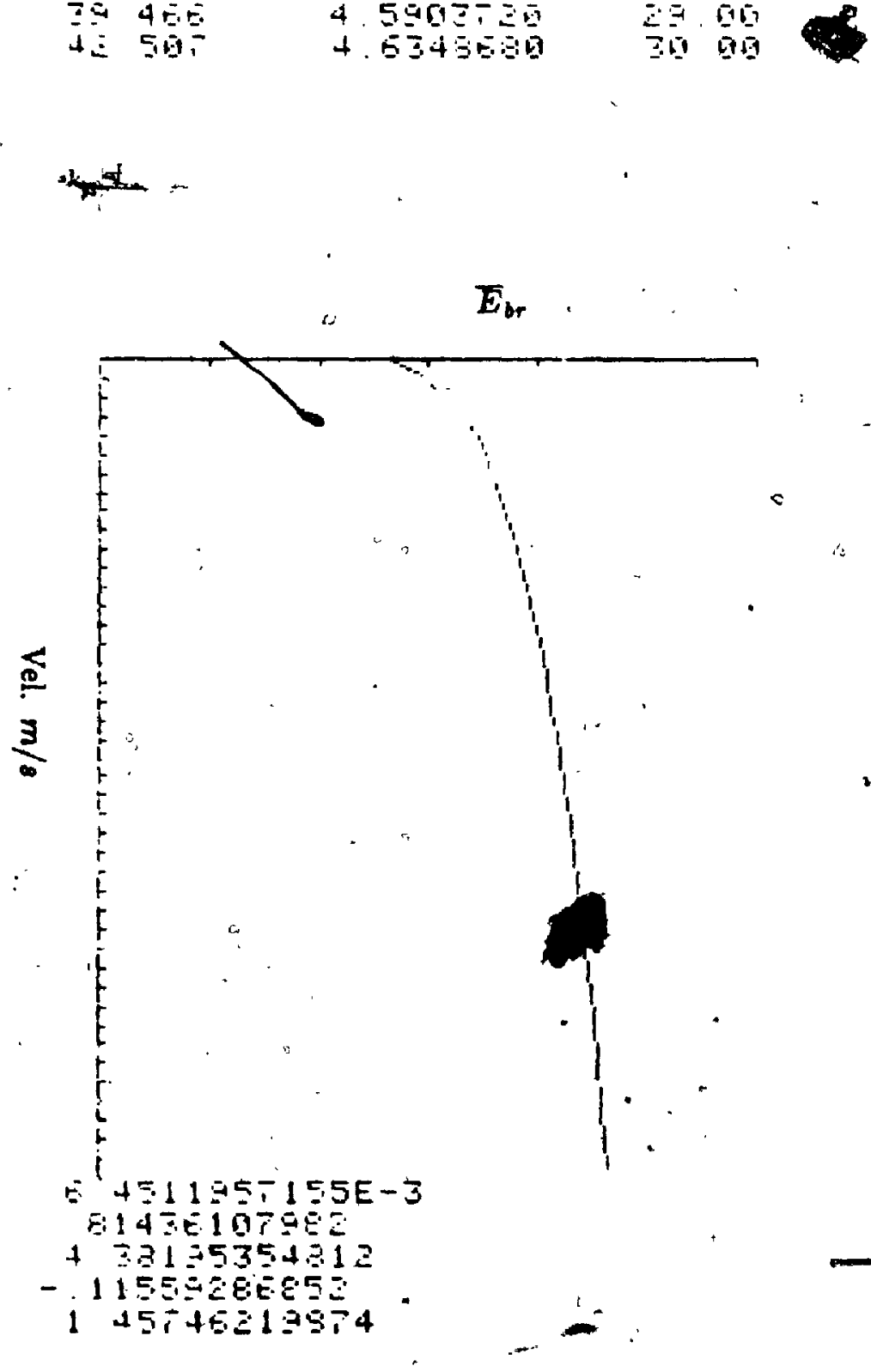


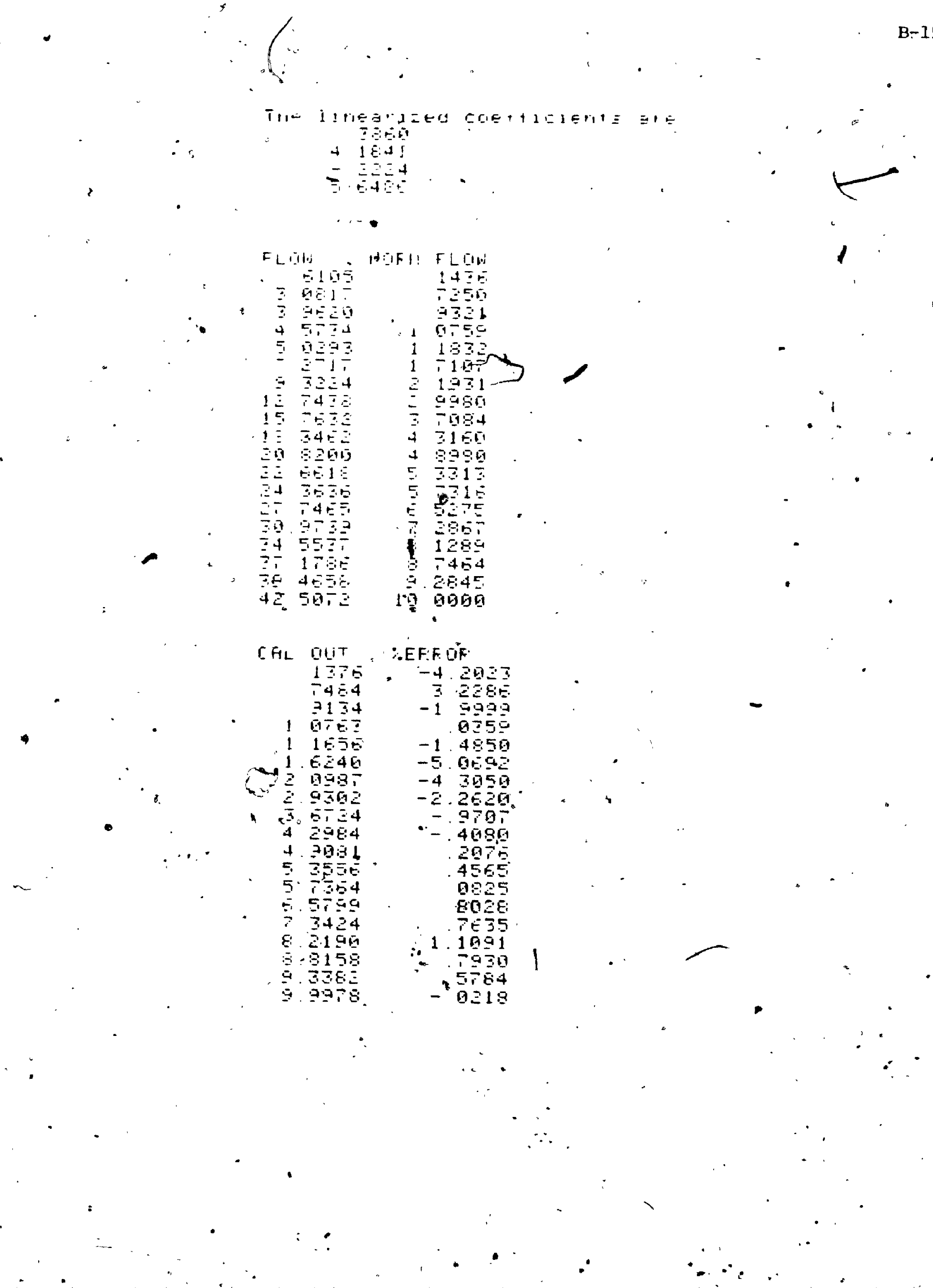



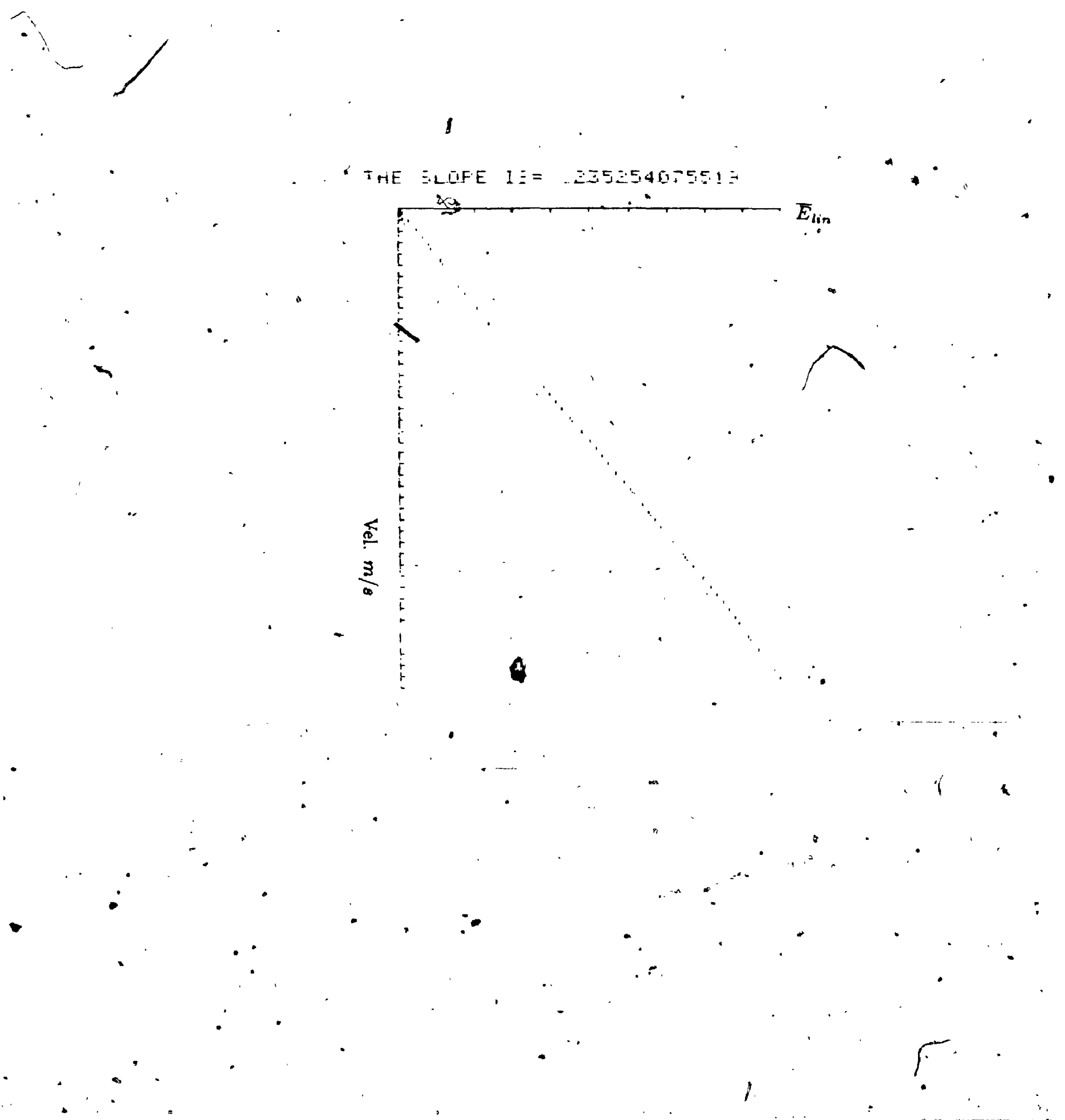


\section{B.2 TSI FORTRAN Calibration Program}

C

C

C

C

c

c:

C

REAL A (8,8), CALFL (21), COEFF (6) ERROR (21), FLOW(21), LINCOEF (4)

$\therefore$ REAL NORMFL (21), POWER (21), R(13), 8 (7), WEIGHT (21), X52 (8,8)

REAL VOLT (21), T(21), TREMP(21)

DIMENSTON $\mathrm{XX}(21), \mathrm{KXX}_{\bullet}(21)$

. C

C

ORDER $=4$

PRINT*, 'ENTER NUMBER OF DATA PAIRS (MAX=21)'

- READ* , NUNBER

PRINT*, NUMBER

C

C

$\operatorname{READ}(5, *)(\operatorname{VOLT}(I), I=1$, NUMBER)

$\operatorname{READ}(5, *)$ (POWER (I) $, \sqrt{I}=1, \mathrm{NUMBER})$

$\operatorname{REND}(5, *)(T E M P(I), I=1$, NOMBER)

IF (I:EQ.1) GO TO 6

IF (VOLT(I) . LT . VOLT (I-1)) PRINT* . ENTER DATA IN ORDER'

c

5 CONTINUE

c

c

c 
13. 18

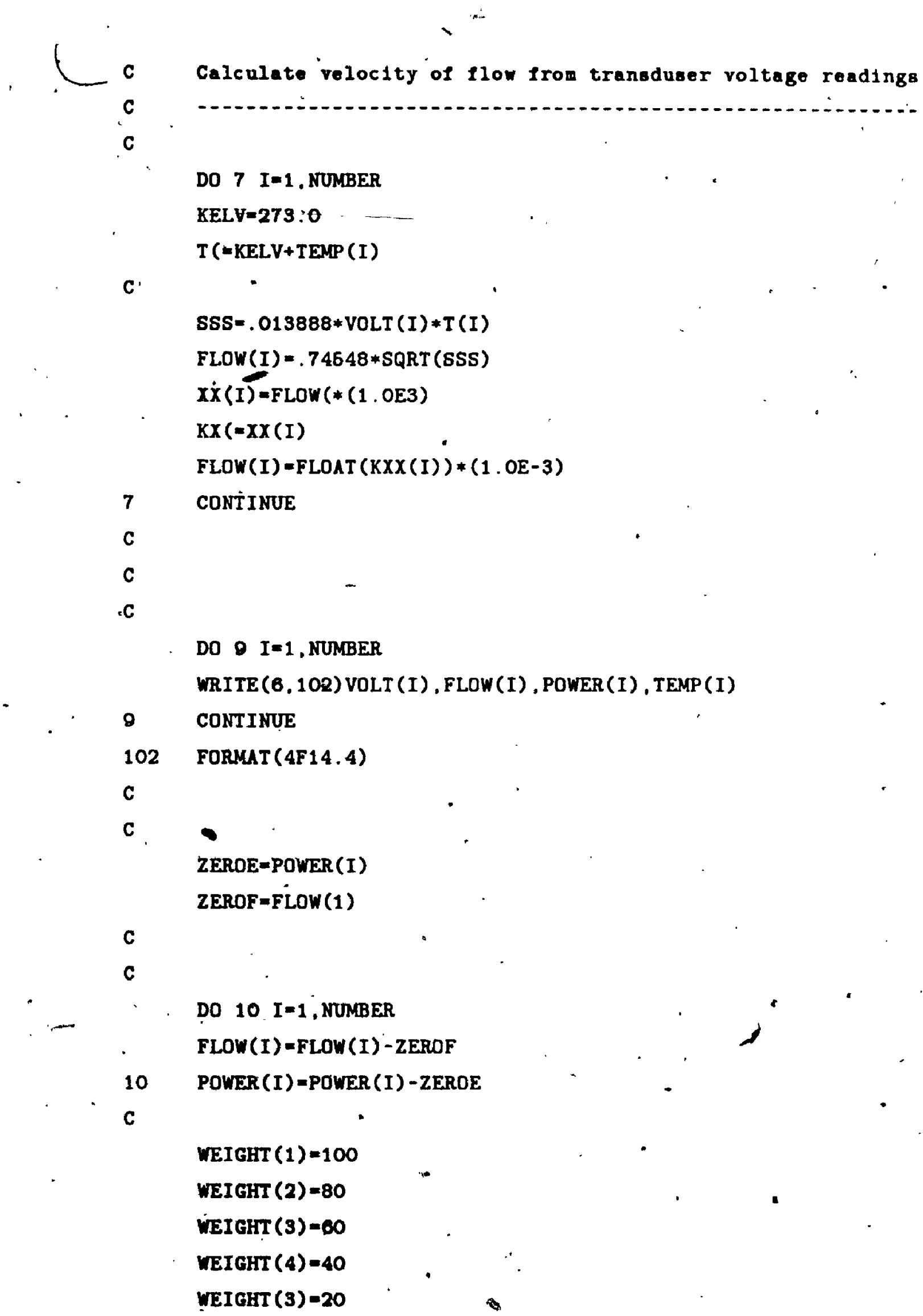




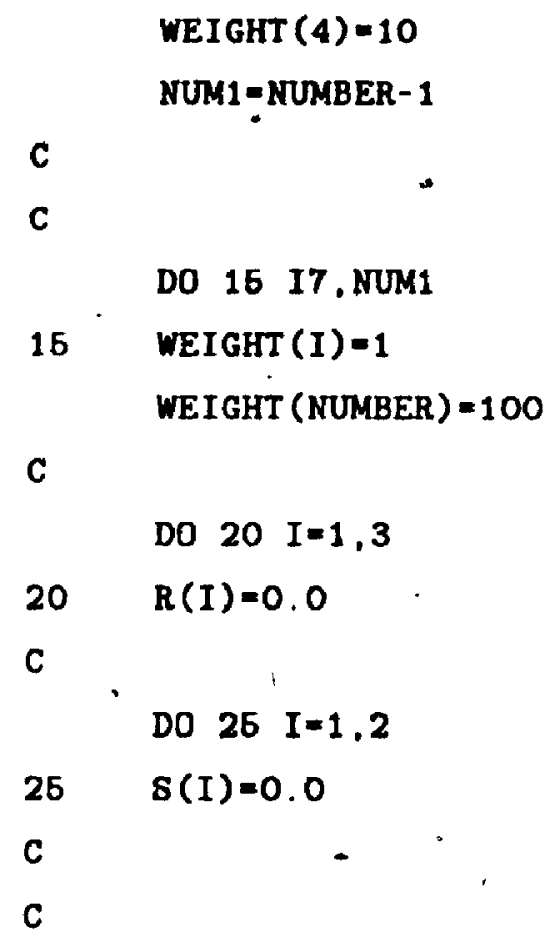




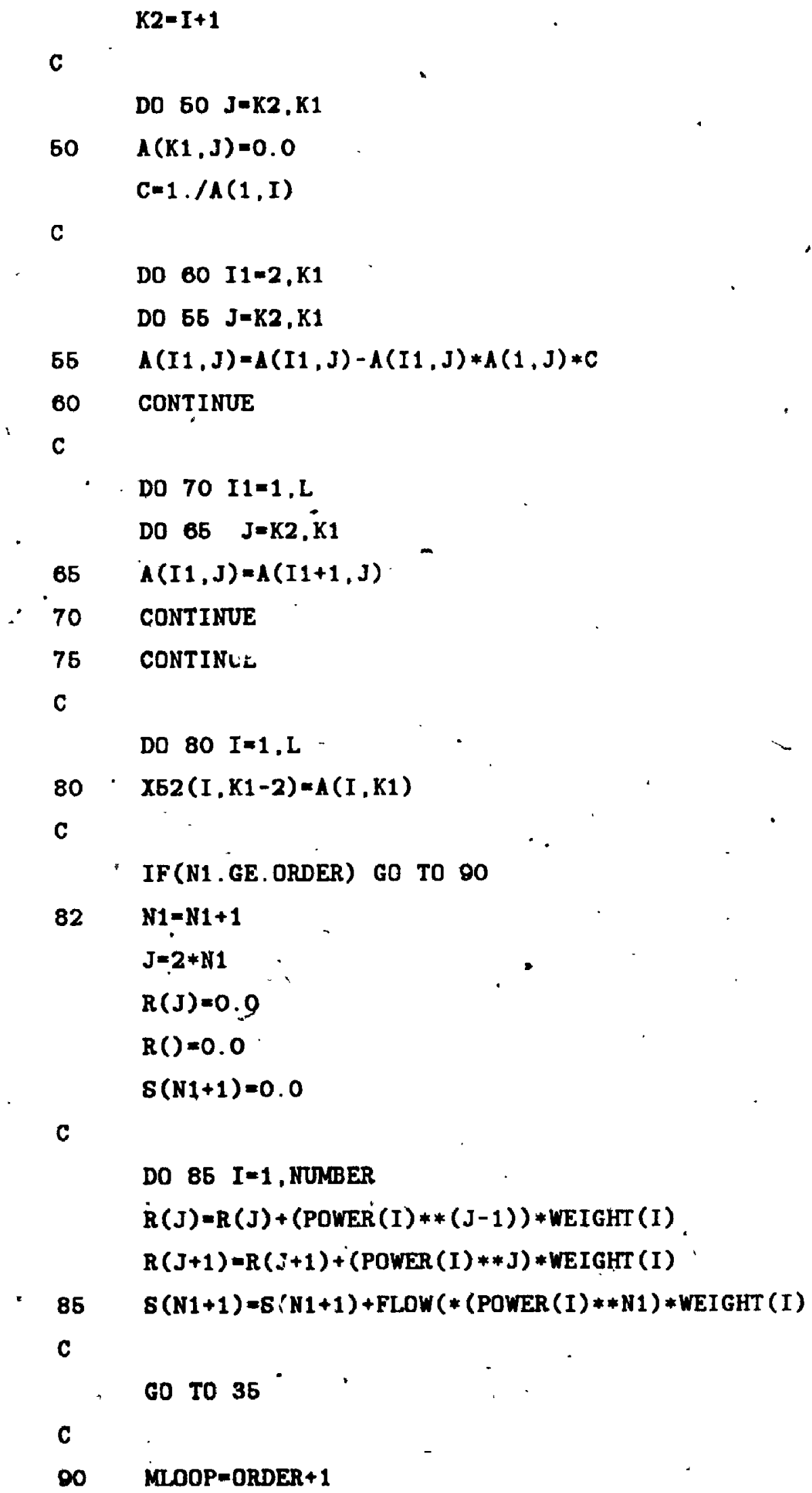


C

DO 06 I=1, MLOOP

$\operatorname{COEFF}(I)=\wedge(I, K 1)$

X62 $(I, 4)=X 62(I, 4) *($ POWER $($ NUMBER $) * *(I-1))$

95 CONTINUE

C

DO $106 \mathrm{JK}=2$. NUMBER

CALFLO $=\operatorname{COEFF}(1)$

C

DO $100 \quad I=2,5$

100 CALPLO=CALFLO+COEFF (*POWER (JK)** (I-1)

CALFL (JK) = CALFLO * 10/FLOW (NUMBER)

C

105 ERROR (JK) $=($ CALFLO - FLOW () *100/FLOW (JK)

SPAN $=10 /$ FLOW (NUMBERR)

C

D0. $110 I=1,4$

ᄀ $\operatorname{LINCOF}(I)=X 62((I+1), 4) * \operatorname{SPAN}$

110 CONTINUE

WRITE $(6,22)$

22 FORMAT ('LINEARIZED COEFFICIENTS')

WRITE $(6,103)(\operatorname{LINCOF}(I), I=1,4)$

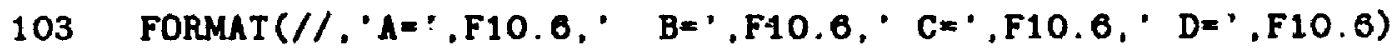

C

C

WRITE $(6,1000)$

1000 FORMAT $(/ .7 X$, 'FLOW', 8X, 'NORM FLOW',4X,3'CALC OUTPUT', 4X, 'PCT ERROR 1$.$) ; ;$

C

DO $115 I=2$, NUMBER

NORAFLOW $(I)=$ FLOW $(I) * S P A N$

WRITE (6, 1001) FLOW(I), NORMFL (I), CALFL(I), ERROR(I)

115 CLWTINUE

1001 FORMAT (F14.5, 2F14.3,F14.2)

C

\section{STOP}

END 


\section{Appendix C \\ Computer Program MFLOW}

The computer program "MFLOW" calculates the mass flow rate across the oritic. liy applying equation 3.11. In equation $3.11, h$ is measured directly while the two correctinn factor multiplier is obtained by interpolation between the values of Table C.1. The Hint density, $w$ is found from measurements of static head and temperature at the high r rensur: orifice tapping.

The velocity at the jet. exit was found by assuming isentropic expansion from plenum to atmospheric conditions. Thus;

$$
\frac{P_{0}}{P_{j}}=\left(1+\frac{\gamma-1}{\gamma} M_{j}^{2}\right)^{\frac{\gamma}{\gamma-1}}
$$

or

$$
\frac{P_{0}}{P_{j}}=\left(1+\frac{\gamma-1}{\gamma} \frac{V_{j}^{2}}{c_{j}^{2}}\right)^{\frac{\gamma}{\gamma-1}}
$$

or

$$
{\frac{P_{o}}{P_{j}}}^{\frac{\gamma-1}{\gamma}}=1+\frac{\gamma-1}{\gamma} \frac{V_{j}^{2}}{c_{j}^{2}}
$$

or

$$
\frac{V_{j}^{2}}{c_{j}^{2}}=\frac{\frac{P_{\mathrm{o}}}{P_{j}} \frac{\gamma-1}{\gamma}}{\frac{\gamma-1}{2}} \quad \text { and } c=\sqrt{\gamma R T}
$$

Then,

$$
V_{j}^{2}=\frac{2 \gamma R T\left[\left(\frac{P_{0}}{P_{j}}\right)^{\frac{\gamma-1}{\gamma}}-1\right]}{\gamma-1}
$$

where

$T=$ plenum temperature $\approx$ temperature of air through orifice

$P_{\mathrm{o}}=$ total plenum pressure

$P_{j}=$ pressure at jet exit

$T$ was recorded with a thermometer downstream of the orifice and $P_{j}$ is the statu pressure reading on the cylinder, $2 \mathrm{~mm}$ downstream of the cylinder jet exit plane. 
Table C.1

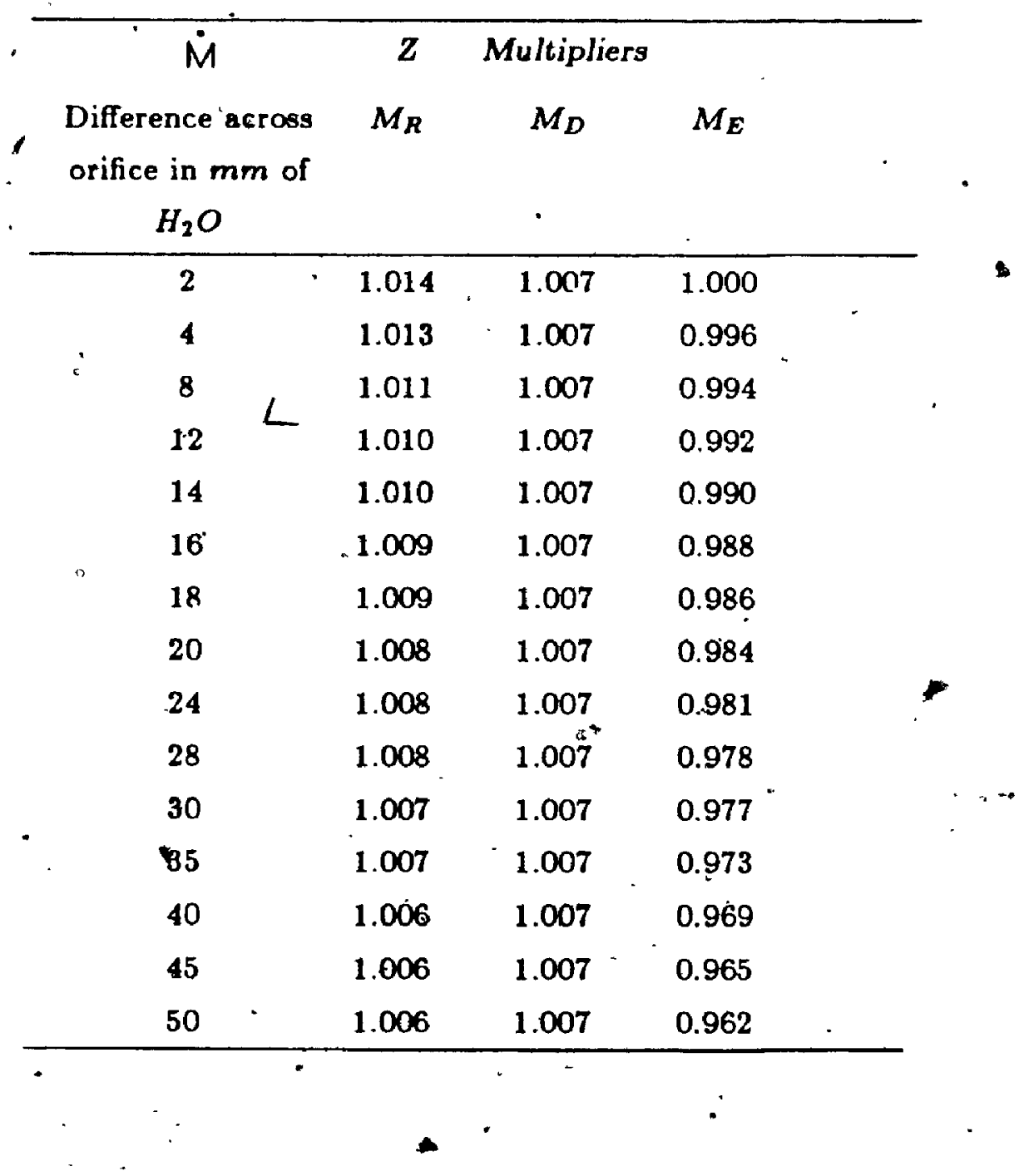

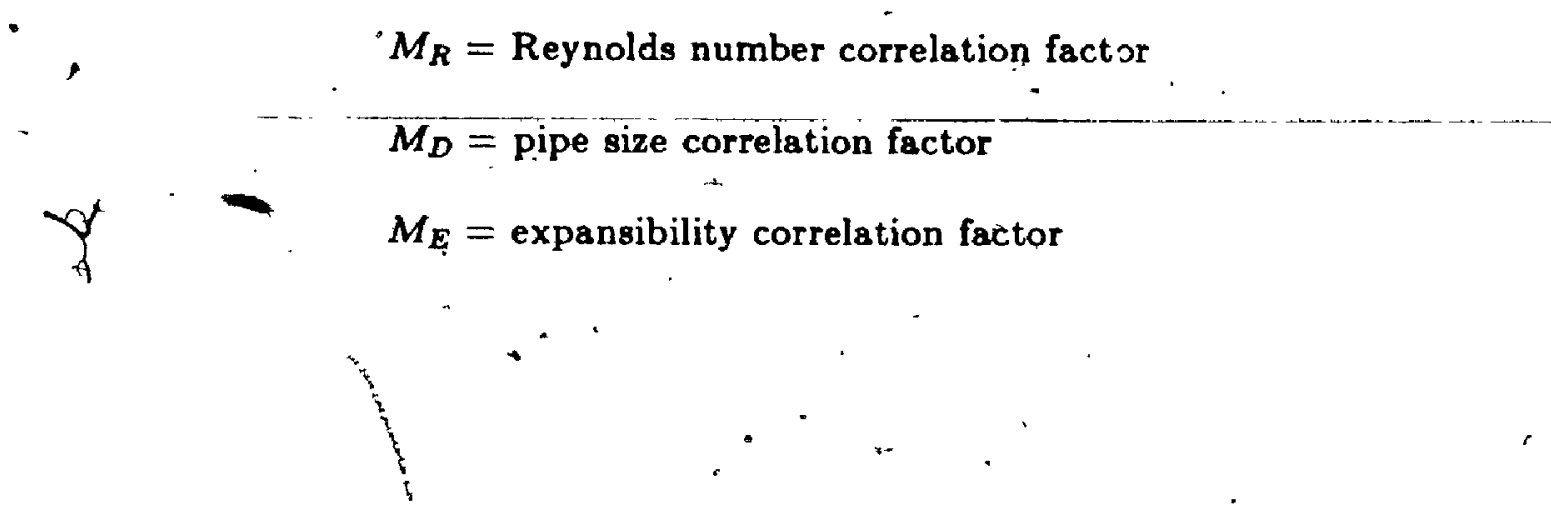


C This program cslculates the MASS-FLow rate through the

C orifice, the extt JET VELOCITY and cylinder SLOT THICKNESS

c

c

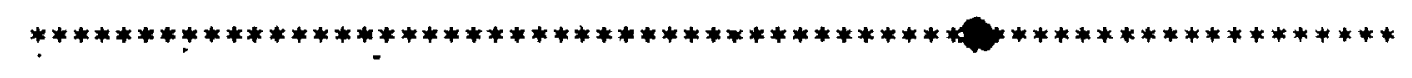

DH ..........head difference across orifice(in H2O)

C

c

$\mathbf{Z}$ Multipliers (from charts)

HHPR ............head at high press. side of orifice(in)

c $\mathrm{T} \quad \ldots \ldots \ldots \ldots \ldots \ldots$ Temp at orifice exit (F)

C $\quad$ HJ $\ldots \ldots \ldots \ldots \ldots$ Head at jet exit (in)

C

PPLEN . . . . . . . Plennum pressure (in).

PATM ............Atmospheric pressure $(\mathrm{Kg} / \mathrm{m} 2)$

c MFR ...............Mass flow rate through orifice $(1 \mathrm{bm} / \mathrm{min})$

C DENSJ ............Air density at jet exit

C Po $\ldots \ldots \ldots \ldots \ldots \ldots$ Plennum pressure

C VJ ............... velocity a't jet exit.

C

TMEAN Mean slot thickness

“ REAL DH, Z, HHPR, T, HJ , PATM, PPLEN, MFR, DENS J , PO , VJ

REAL DENS ; TMEAN, VEL, PTUB, FLAG, T JK, MFRM

FLAG $=1$

C

28 REÀD $(5, *) D H, Z, H H P R, T, H J, P A T M$, PPLEN , PTUB

C

c

TEMP $=T+459.69$

DENS $=(2, *$ HIPR $* 5.2+14.7 * 144) /(63.3 *$ TEMP $)$

$M F R=6.477768168 * Z * \operatorname{SQRT}(\mathrm{DH}) * \mathrm{SQRT}$. (DENS)

C $\quad$ MFRM $=M F R * .00756$

:PRESJ $=\mathrm{HJ} * 25.400+\mathrm{PATM}$ 


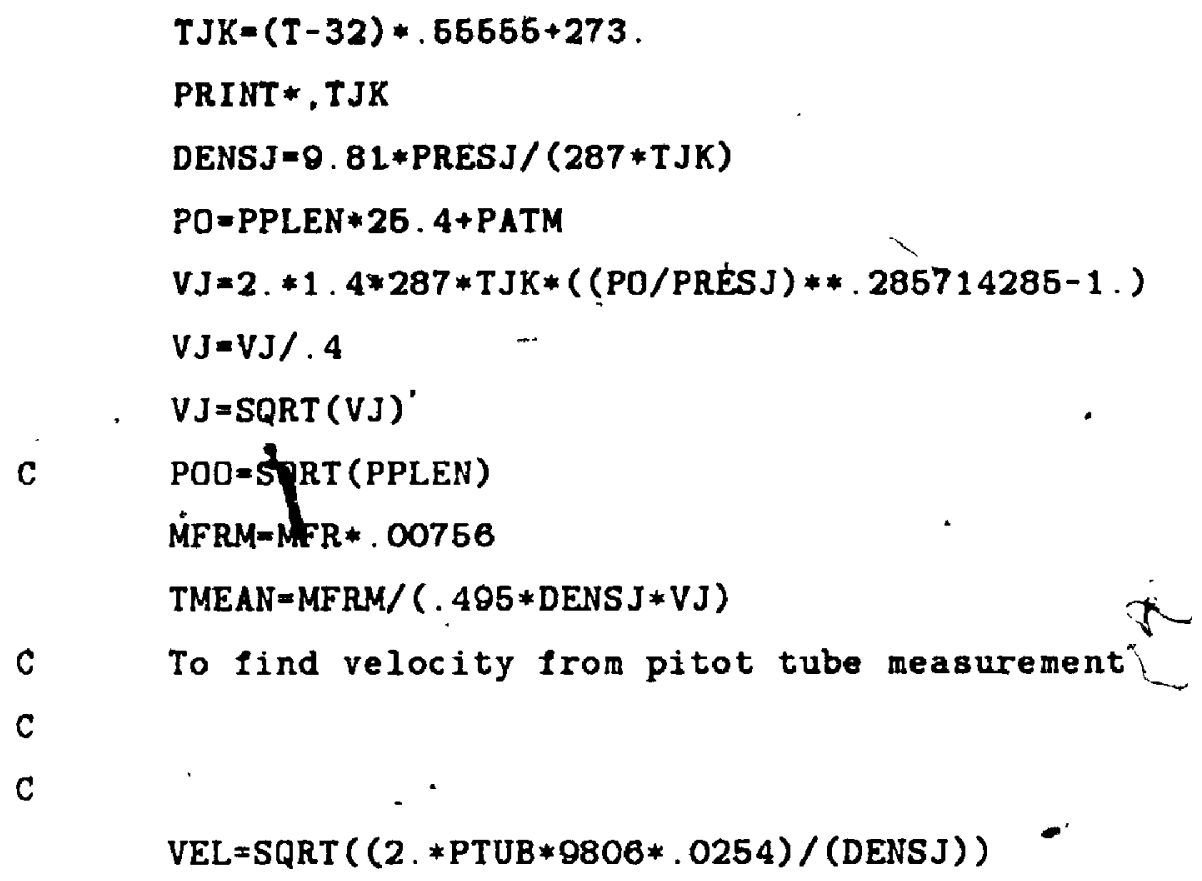

10 FORMAT('The HEAD difference across ortsice is:') WRITE $(6,20)$ AH

20 FORMAT (F10.4)

WRITE $(6,40)$

40 FORMAT $(/ /)$

WRITE $(6,1)$ DENS , MFR

1 FORYAT('DENSITY=',F10.6,6X, 'MASS FLOW=' F10.5) WRITE (6, 2) DENSJ, VJ

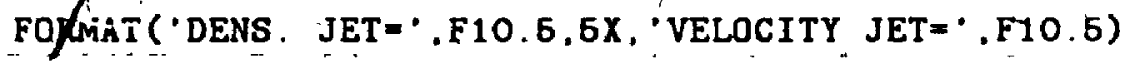
WRITE $(6,30)$ VEL

30 FORMAT ('VELOCITY P. TUBE=', F10.6) WRITE $(6,4)$ MEAN

4 FORMAT ('MEAN=', F10.6)

FLAG $=$ FLAG +1

IF (FLAG .LE. 9) COTO 28

STOP

END 
A brief description of the program, U\&RMS, and its statements are given below along with the flowchart and the program listing. The computer program, M\&RMS, is a slightly modified version of the program, U\&RMS. The M\&RMS program is used for sampling the hot-wire signals with a slanted probe and outputs the distance, $Y(\mathrm{~mm}), \bar{E}_{b r}$ and $\sqrt{\overline{e^{2}}}$. The program, U\&RMS, samples the hot-wire signals when a normal probe is used and outputs $Y(\mathrm{~mm})$, calculates the mean velocity, $u$, according to equation 4.15 of Chapter 4 and $\sqrt{u^{\prime 2}}$ according to equation 4.18 of Chapter, 4 .

Because the two programs are identical in all' otheraspects, listing of the program, M\&RMS, is not included.

\section{'Description of U\&RMS Program}

6

Parameter

Description

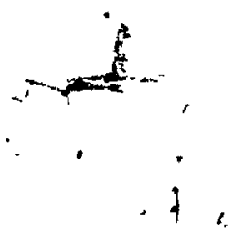

$w 9 ; w 1 ; w 3 ; w 2$ Distances $O G ; O A ; A W ; O W$ in $\mathrm{cm}$ to be used in traversing gear kinematic analysis

$k 1$

$A 9$ Initial number of traversing gear steps

No Actual guide tube advance for initiál gear steps specified

$N$ Initial number of data points to be taken

$A 2 ; A 1$ Total number of data sets

Input number of times that the HP $3456 \mathrm{~A}$ voltmeter will be triggered

- for RMS measurements; and the corresponding number for mean voltage measurements

$A O ; B ; C ; D ; C$ Coefficients determined from program "CALIB*n to be used for linearization of signal

$S$. Siupe of linearized $\bar{E}_{\text {lin }}$ v\$ $U$ curve, determined from program, "CALI\&"

\section{$P 6 ; T 6 \quad \psi$ and $\alpha$ angles of probe}

T5

\section{Measured angular distance from cylinder stot}

$B 5$ Cylinder momentum coefficient, $C_{\mu c}$

$P O(\cdot) ; F O\left(\right.$ ) $\bar{E}_{l i n ;} \sqrt{e^{12}}$ 


\begin{tabular}{|c|c|}
\hline $250-280$ & Date of experiment \\
\hline $320-390$ & Input traversing gear dime:nsions \\
\hline $430-610$ & Specify the initial traversing gear size \\
\hline $750 \cdots-840$ & Input the number of data points per set and number of sets \\
\hline $850--950$ & $\begin{array}{l}\text { Specify how many times the HP } 3456 \mathrm{~A} \text { voltmeter has to be triggered for } \\
\text { measuring RMS and mean voltages }\end{array}$ \\
\hline $980-1140$ & $\begin{array}{l}\text { Input coefficients and slope found from program, "CALIB*", to be used } \\
\text { to linearize signals }\end{array}$ \\
\hline $1170-1360$ & $\begin{array}{l}\text { Specify the probe orientation, the measuring station and the cylinder } \\
\text { momentum coefficięt }\end{array}$ \\
\hline $1420-1780$ & Loop to sample signals \\
\hline $1800-2050$ & $\begin{array}{l}\text { Traversing gear kinematic analysis to determine actual probe distance, } \\
Y(\mathrm{~mm}) \text {, at each point of the flow }\end{array}$ \\
\hline $2060-2360$ & Relay actuator software to control movement of probe \\
\hline $3390-3650$ & $\begin{array}{l}\text { Subroutine "Avdev" to sample mean voltages at the rate specified in } \\
\text { Chapter } 3\end{array}$ \\
\hline $3650-3850$ & $\begin{array}{l}\text { Subroutine "Acv" with modifications to sample RMS signal at the rate } \\
\text { specified in Chapter } 3\end{array}$ \\
\hline
\end{tabular}

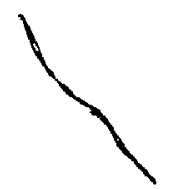




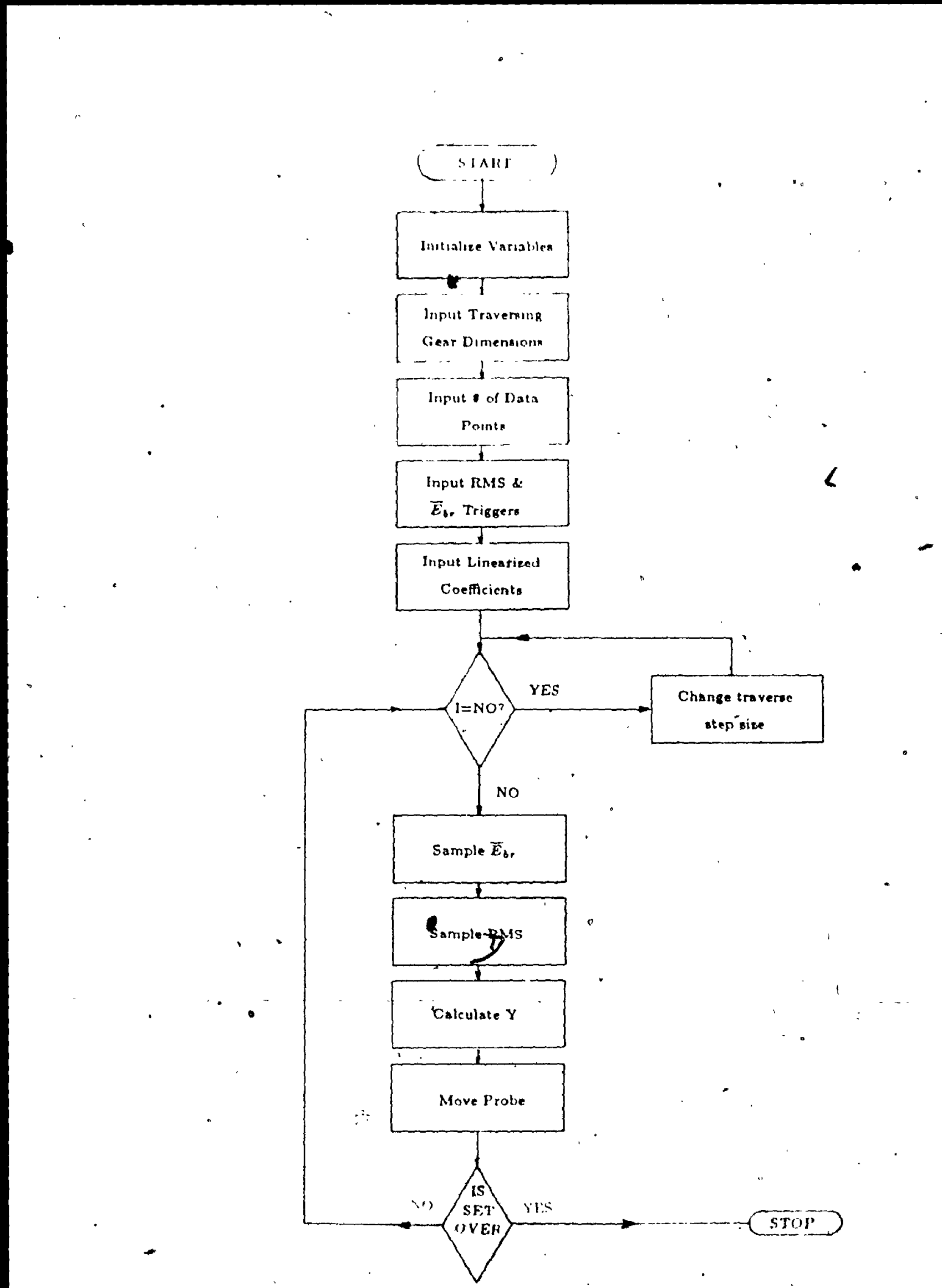

Figure D1: Flowchart of Computer Program U\& RMS 


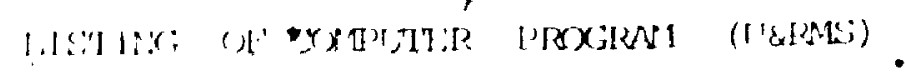

a

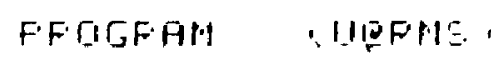

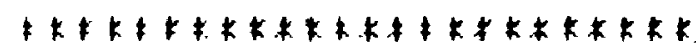

THIE PEOGFAM EFLEULATES

MEAII AND TURBULENT

[IJPHA T TES [ISINS

H PIJPIAL $H$. W PRDBE

Itume.25.55.

$1 \frac{1 \mathrm{~B}}{12}$

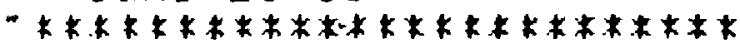

1 渵

$1+4$

159

156

$1 \div \overline{1}$

DHAHEL 2 MEASUIRES (FMS)

18.9

CHAHEL \& MEASURES (MEAH)

290

PEAL

Q1,09,W1, W2.W3,Pg.M1

$21 \overline{1}$

REAL H.4.QZ.FE.MG.AG.ME

2दि

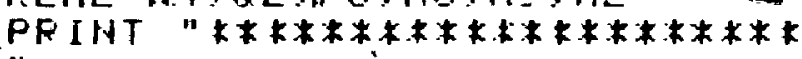

¿30 PFIHT

E49 FRINT "Mean E Long. Turbulenc E Frofile"

Eラ UIJP "DATE IS"

EQT INFIIT DIO

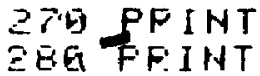

ZgG: PRINT

"IULr-":09;"-85"

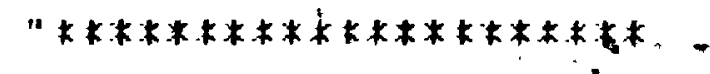

3Q1日 PFIHT

31 FFINT

32 PPINT "Trawersifíg Gejar Dime nEion三.cm;"

33 PRIHT

$3+9$ PRIMT

35 FFIIHT

35g PRINT "OA=" Q IHPUT. HIE PRIH

$"$

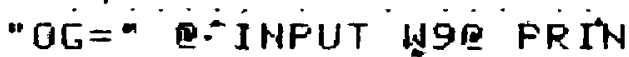
$T W 1$

- 370 PRIHT" "AW=" E IHPUT WJE PRIH 339 PRINT "OW=" Q INPIJT W2P PRIHA

$T$ HE

İG PRINT Q PRINT

AGE PFIHT

4131

$4 \bar{C} \quad 1$

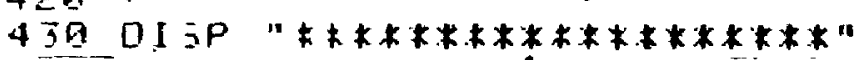

440 OISP "Fieadx to' input the ini

t al number of

45 DISP. "for each (Y. stef)"

tog DISP " $i \mathrm{i}$ e how mapo aejor ste

- FE rols want before you move"

$4750 I 3 p "$ to the next meastaring FOS1+10n,"

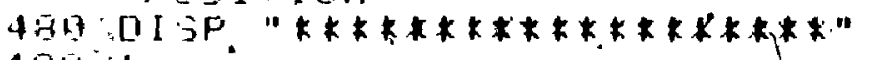
4 व的 


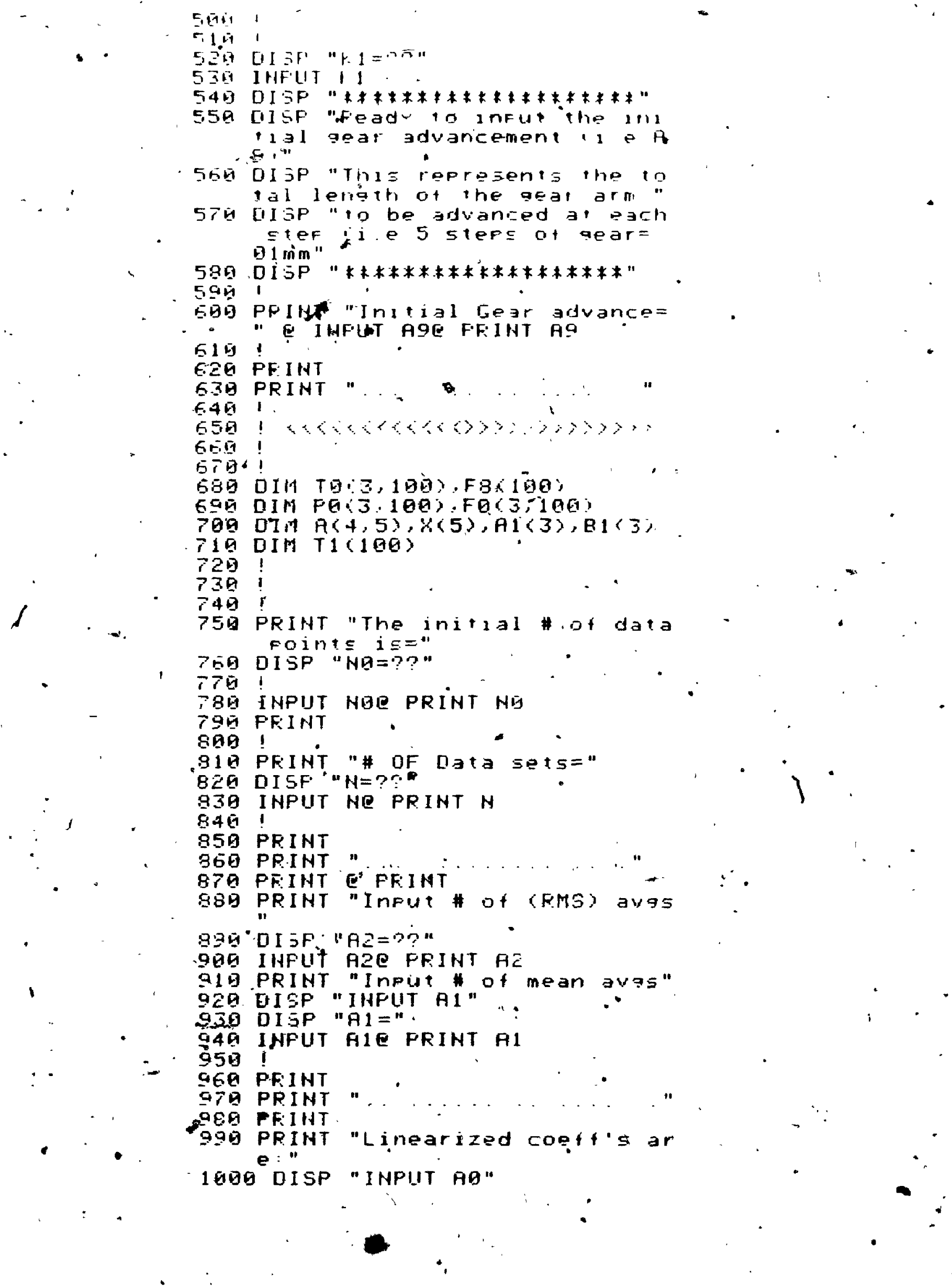




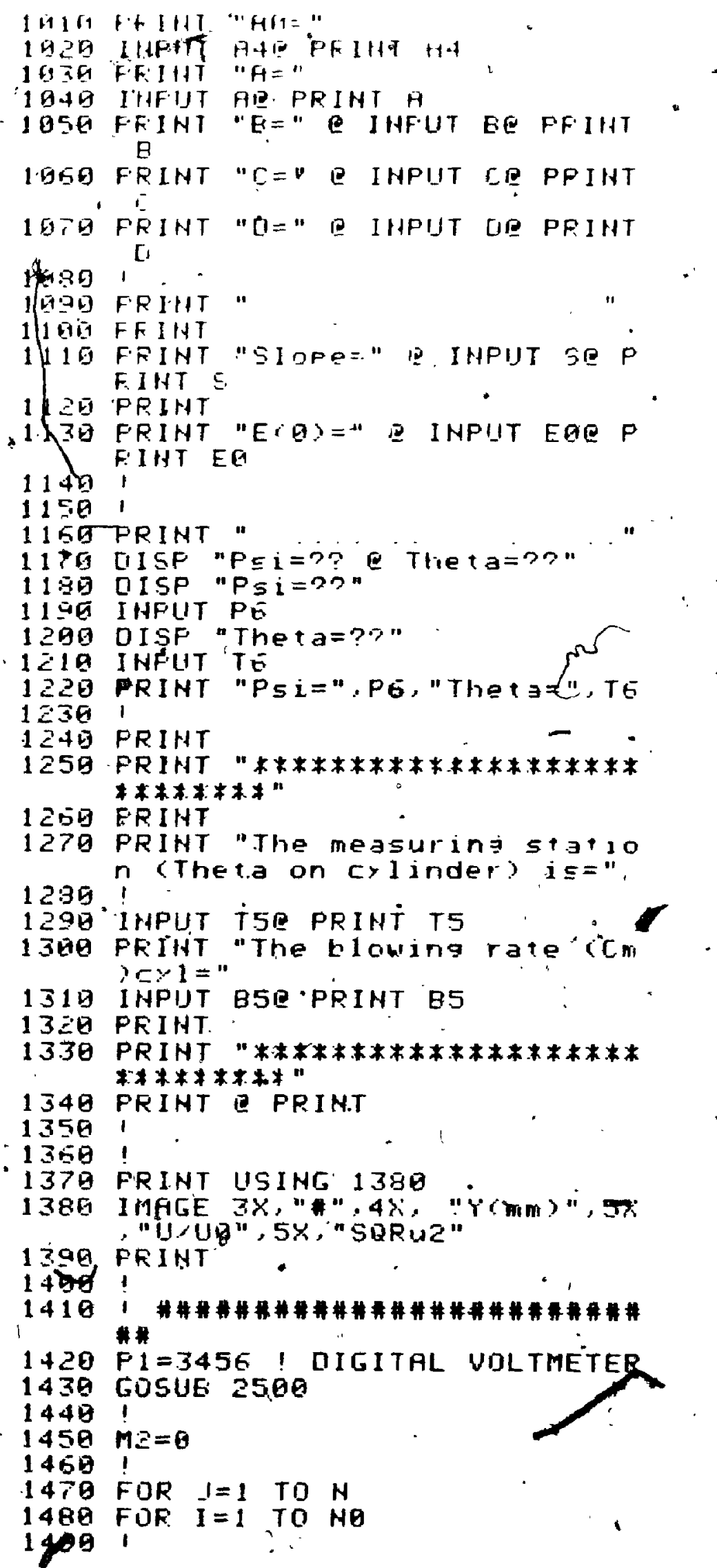




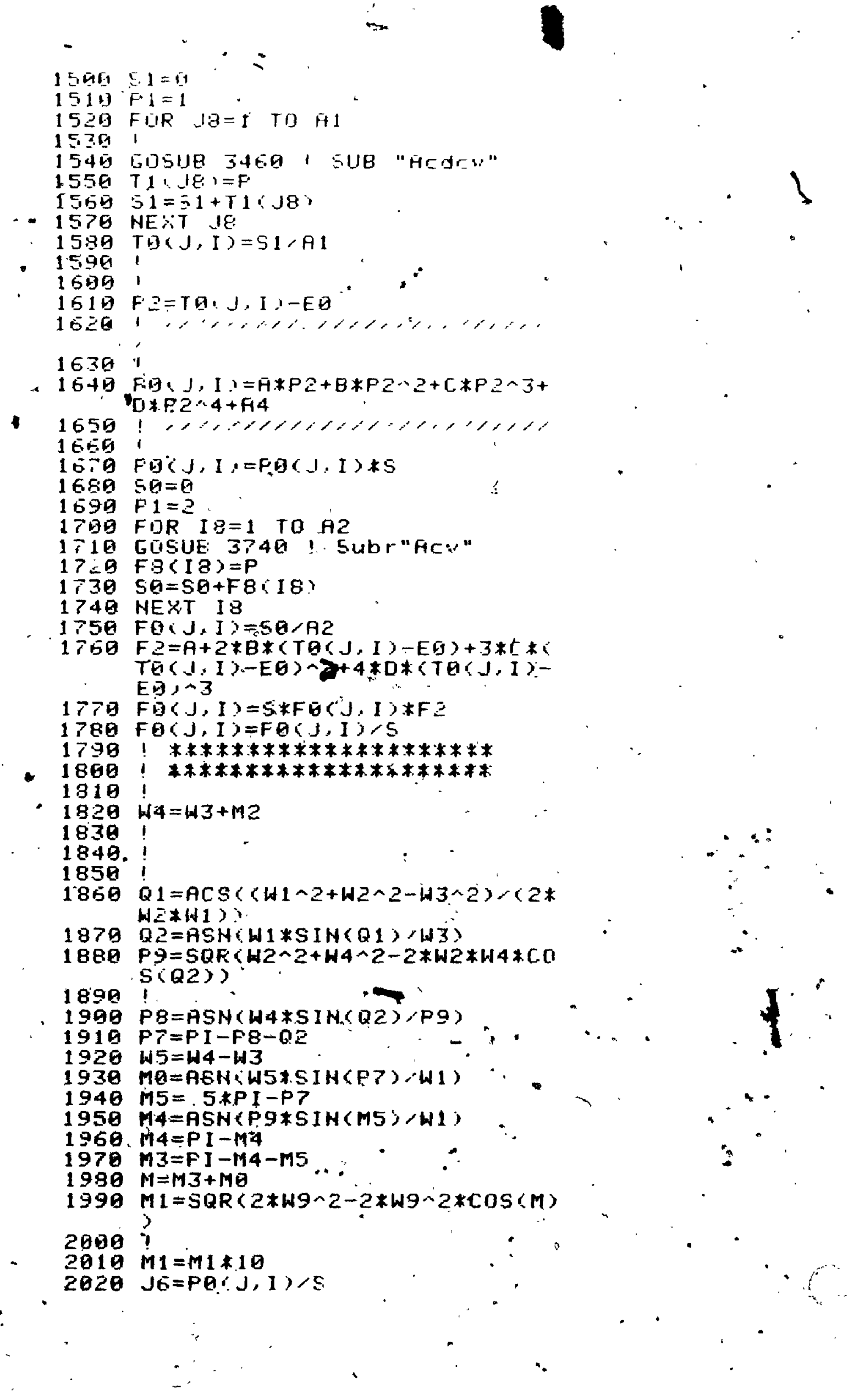




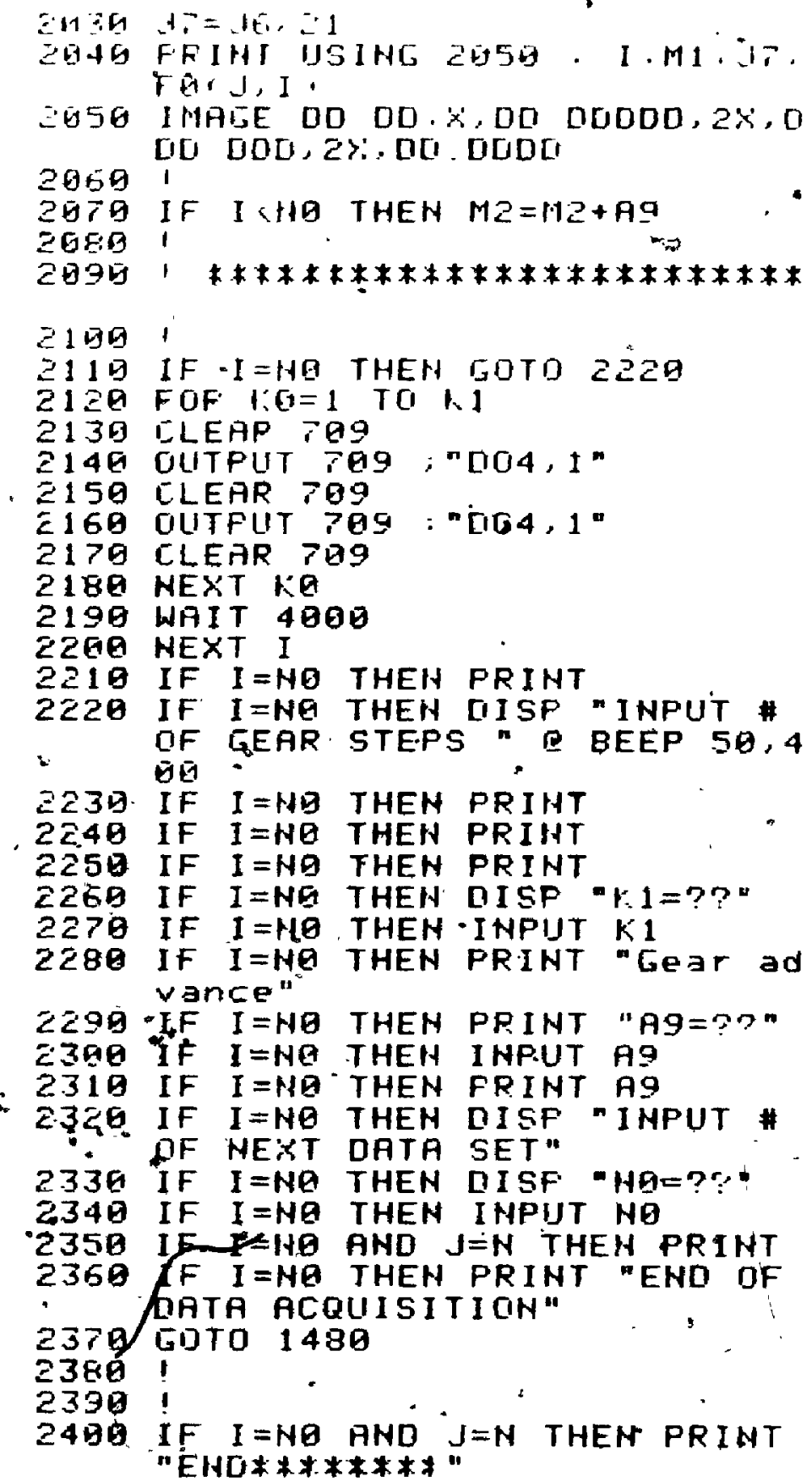




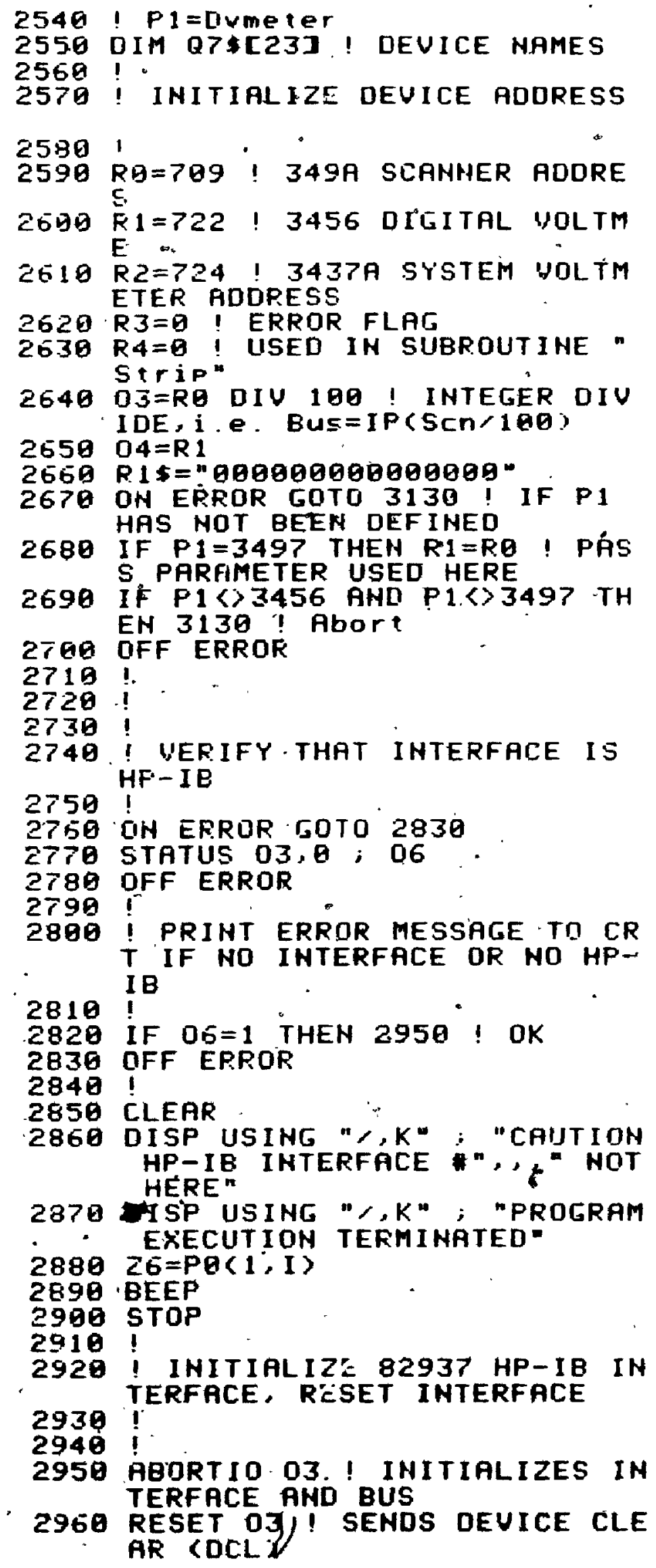




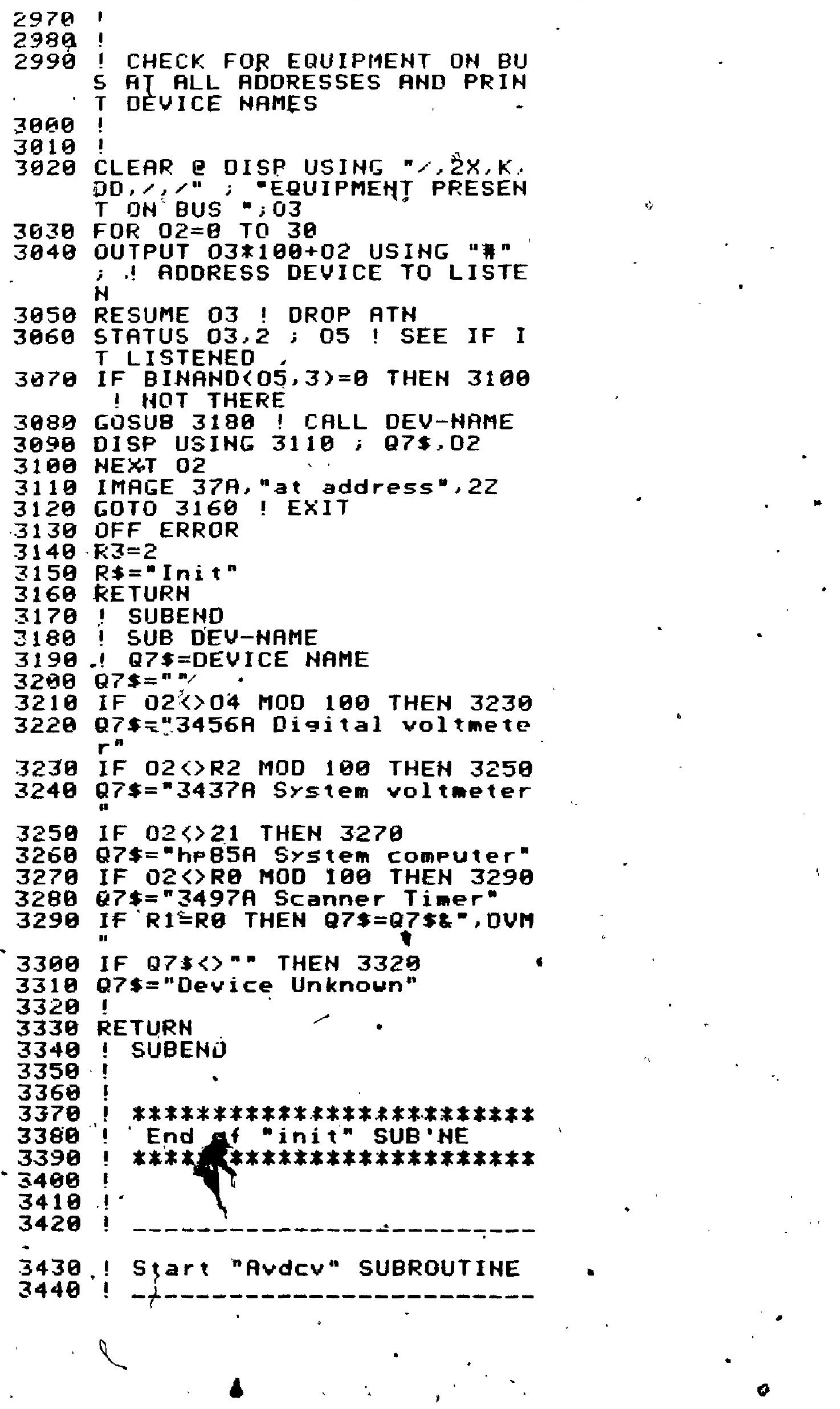




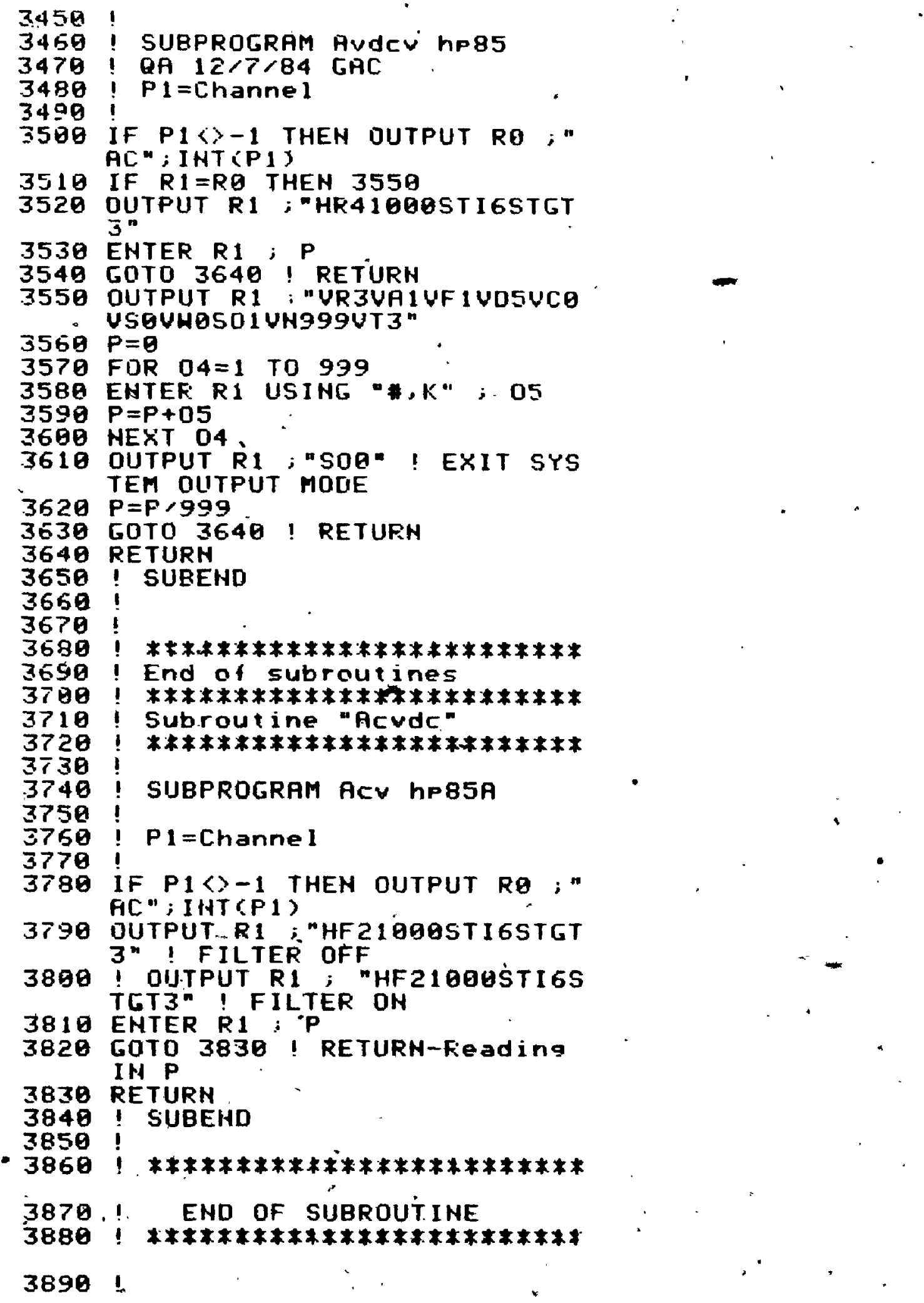


SAMPLE OUTPUT FROM COMPUTER PROGRMM (UERMS)

D-1:

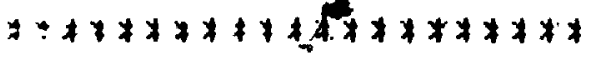

HIJII- $=-E 5$

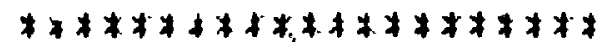

Trawersung Gear [umensions iclu'

$\square \bar{G}=$

4:. $8 \mathrm{E}$

$\overline{\mathrm{H}}=$

$10 \cdot 35_{1}$

$\tilde{T H}=$

हैं $\overline{1}$

$\nabla W=$

$2 \bar{t}+4$

Iritisi Gear jovirice

The luitis \# of datapoints is=

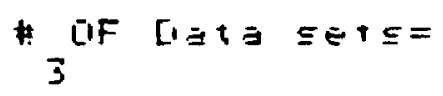

Irifut \# Gi iRME aves

Irifut of mean aves.

$\rightarrow$

Liriearized coeff's are

$A \bar{b}=$

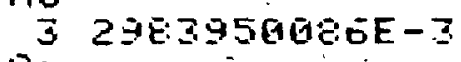

$A=$

$\mathrm{B}=95021823251$

G 2939511398

$-3.47118457849$

0= 27892285316

$2878 \pm 2285316$
$\ldots \ldots$

SIOFE $=$

183909385757

$E(\theta)=$

¿ 7202735

$\dot{P} \mathrm{si}=$

Thieta=

$\therefore$

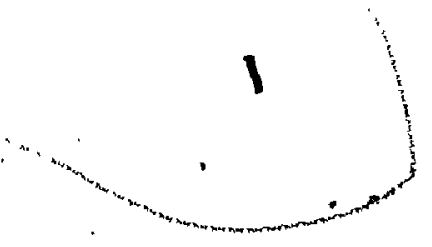

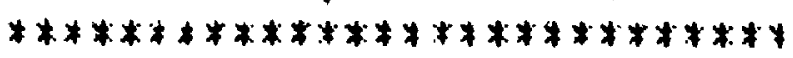




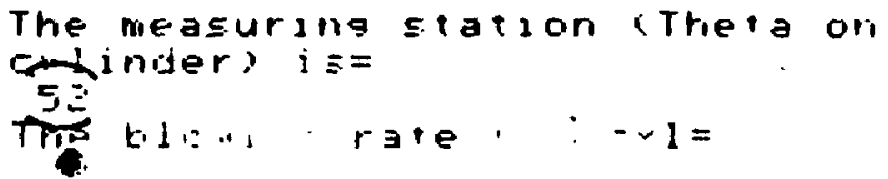

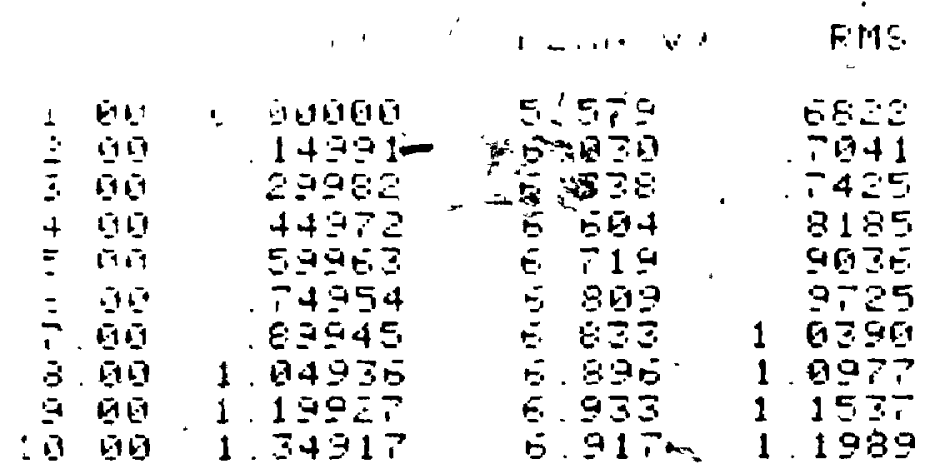

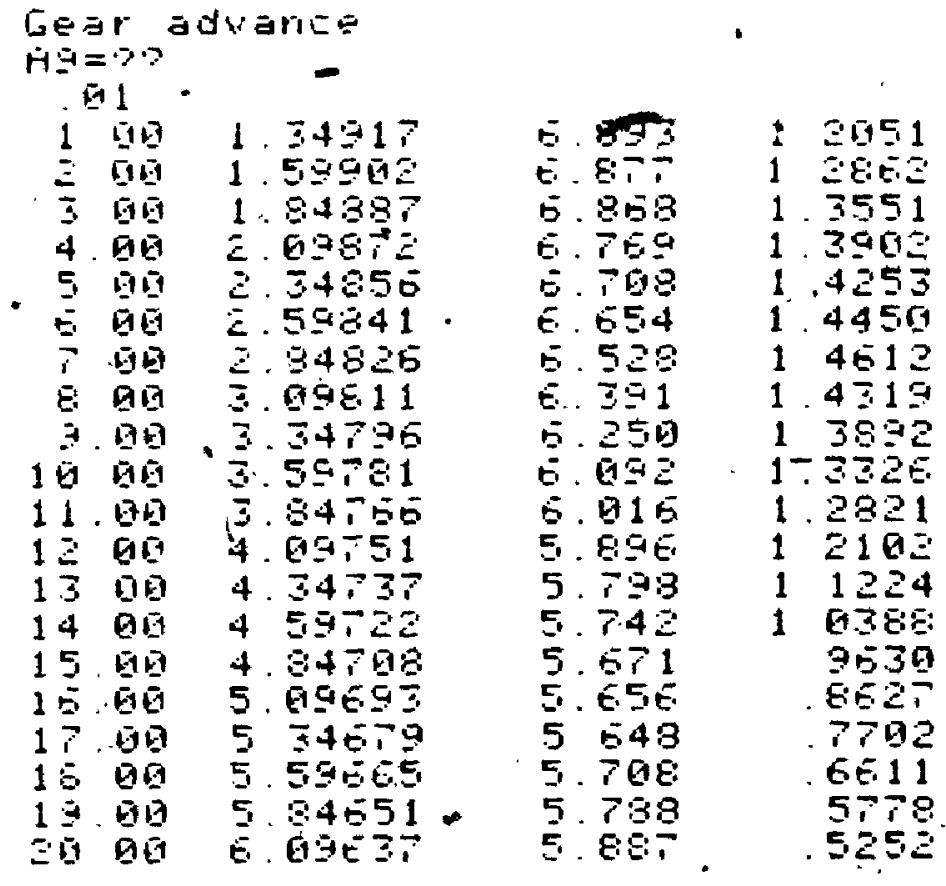

GE三* adwan=E $\Xi \cdots$

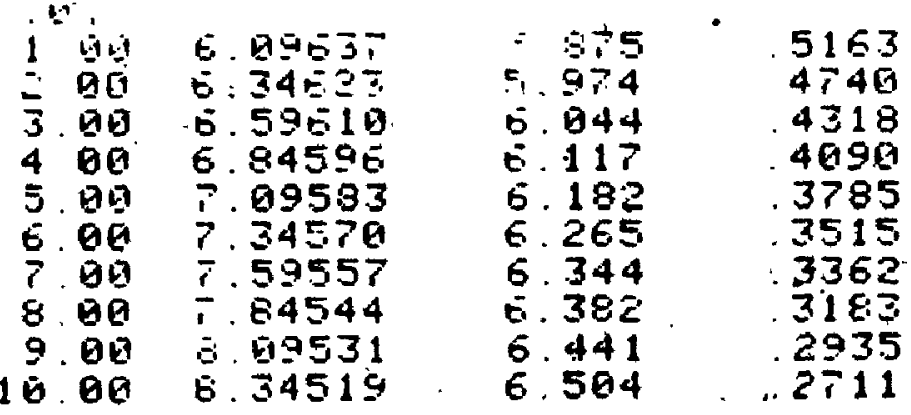




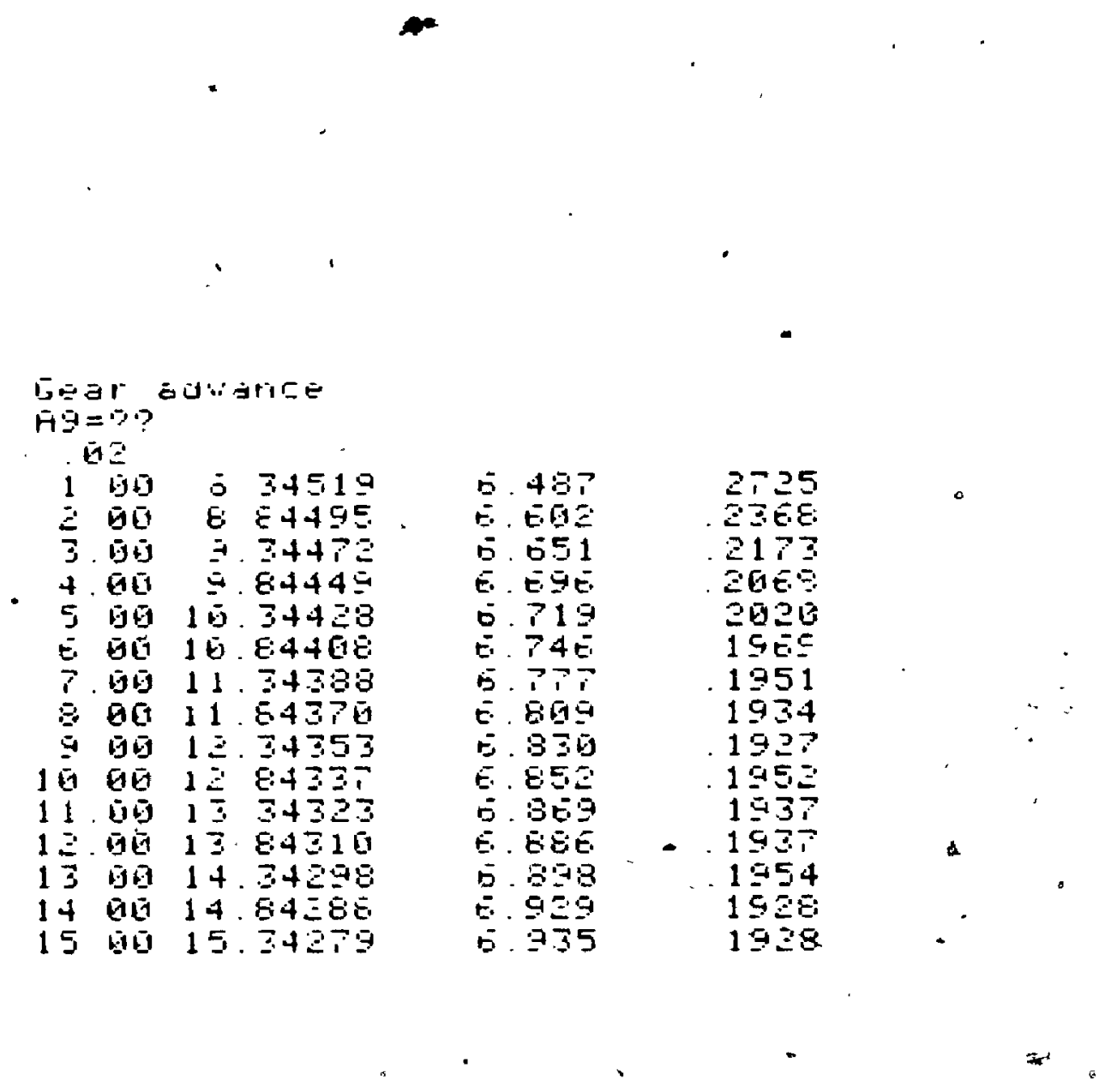




\section{Appendix E \\ Hot-Wire Signal Analysis Programs}

C
$C$
$c$
$c$
$c$
$c$
$c$
$c$
$c$
$c$
$c$
$c$

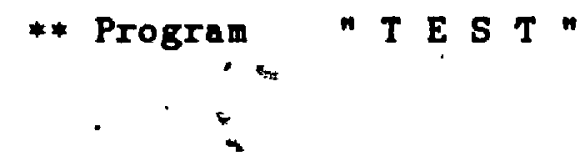

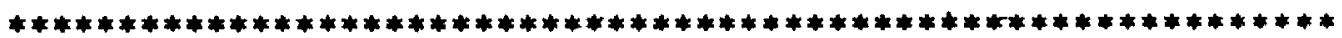

This program tẹsis H.W signal analyais derived in section (4.2). Equations are solved in a number of different ways.

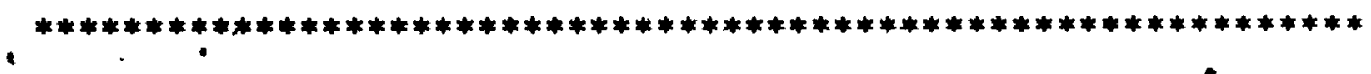

.

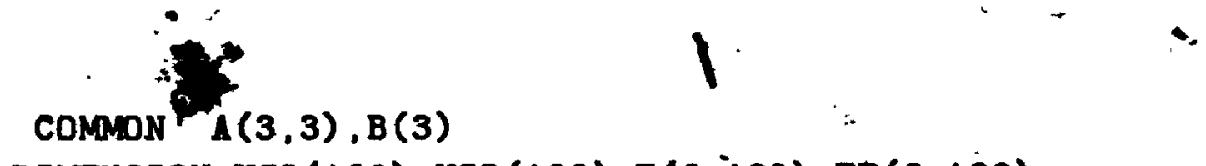

- DIMENSION USQ (100), VSQ (100), E(6,100), EF (6,100) DIMENSION $\operatorname{EL}(6,100), \operatorname{EFL}(6,100), \mathrm{U6}(100), \mathrm{VE}(100)$

DIMENSION U(100), V(100),W(100), U2S (100), V2S (100), U2N (100) DIMENSION UVX (100), V2N (100)

REAL K, H; S, UVZ, UWZ, U2Z, W22, V2Z, V22, AO, A1, B1, D1 , EO

REAL KN, HN, SN, MA1, AM2, AA3; BB1 ; BB2, BB3 , QQ1, QQ2, QQ3, PAR

- REAL PARN,C1,C2,C3, AON,A1N,B1N,C1N, D1N, EON, PARNN,BB4 .

INTEGER NO:

C
C

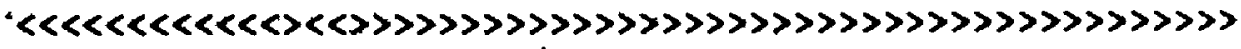

PRINT*, 'ENTER NUMBER OF DATA POINTS (max 100)'

READ*, L

PRINT*, 'ARE SIGNALS LINEARIZED ??'

PRINT*. 'IF YES (OPTION=1)'

READ*,OPTIDN

IF (OPTION. NE. 1)GD TO 38

C

c

c

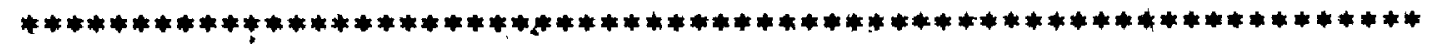
C 


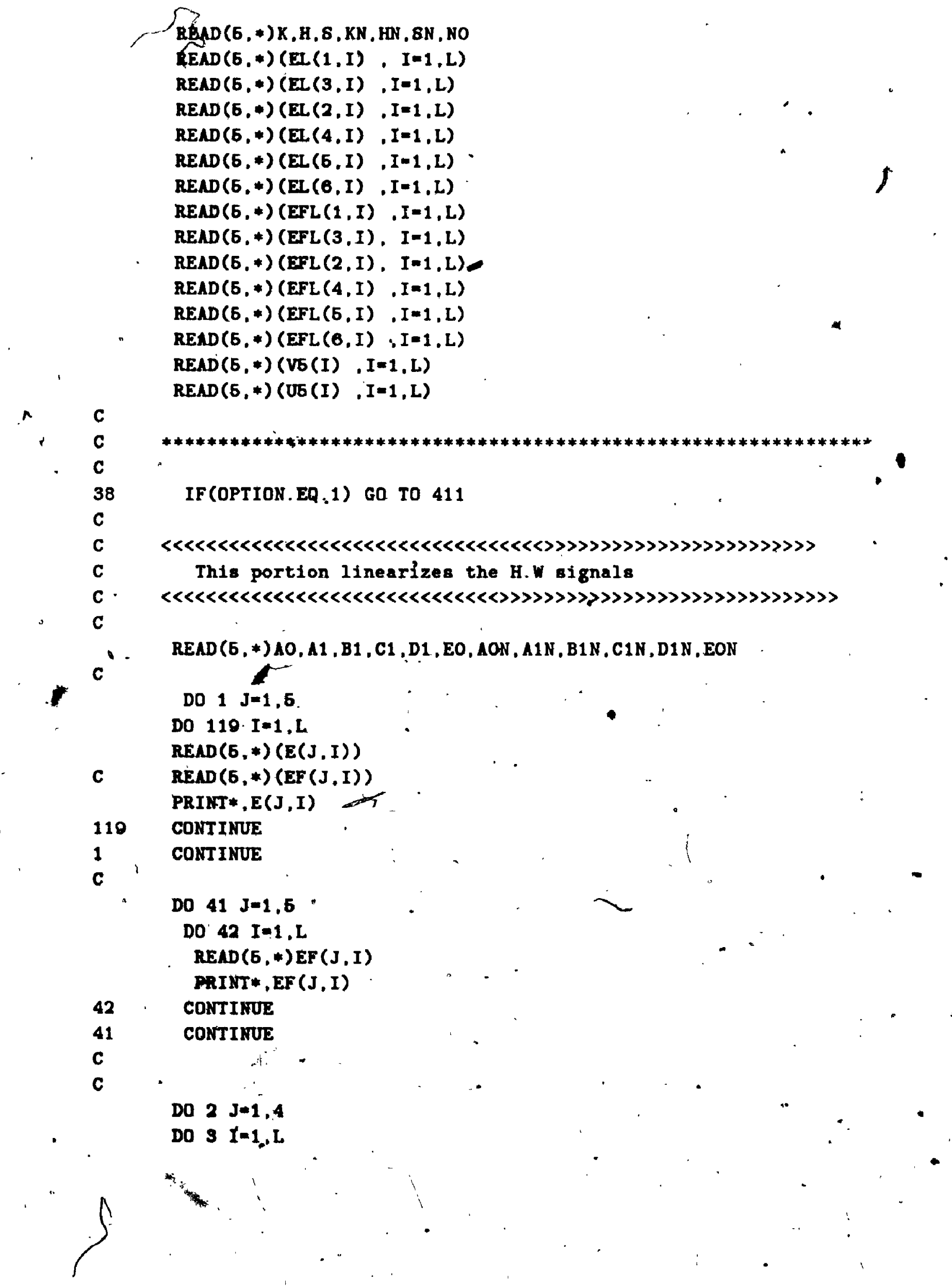


$E L(J, I)-i 0+A 1 *(E(J, I)-E O)+B 1 *(E(J, I)-E O) * * 2+C 1 *(E(J, I)-E O) * * 3$ $1+D 1 *(E(J, I)-E 0) * * 4$

$$
E L(J, I)-E L(J, I) * 8
$$

C

$E F L(J, I)-41+2 . * B 1 *(E(J, I)-E O)+3 . * C 1 *(E(J, I)-E O) * 2+4 . * D 1 *(E(J$. 1I) $-E O) * * *$

$\operatorname{EEL}(J, I)=\operatorname{EFL}(J, I) * S * \operatorname{EF}(J, I)$

WRITE $(6,2002) \operatorname{EL}(J, I), E F L(J, I)$

FORMAT (2I, "EL (J, I) n,:F10.3,2X, "EFL (J,I)",F10.3).

CONT INUE

CONTINUE

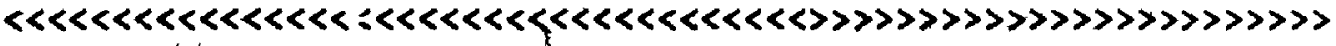

(c)

a.

End of Linearization (Mean voltages)

C

C

C

DO $61 \quad I=1, L$

$E L(5, I)=A O N+A 1 N *(E(5, I)-E O N)+B 1 N *(E(5, I)-E O N) * * 2+C 1 N *(E(5, I)-$ $1 E O N) * * 3+D 1 N *(E(5, I)-E O N) * * 4$

$E L(6, I)=E L(6, I) * S N$

$\operatorname{EFL}(5, I)=A 14+2 . * B 1 N *(E(5, I)-E O N)+3 . * C 1 N *(E(5, I)-E O N) * 2+4 . * D 1 N$

$1 *(E(5 . I)-E O N) * * 3$

$\operatorname{EFL}(5, I)=L L(5, I) * \operatorname{SN} *$.EF $(5, I)$

WRITE $(0,2001) \operatorname{EL}(5, I), \operatorname{EFL}(5, I)$

2001 FORMAT (2X, "EL (5, I)" , F10,3,4X, "EFL(6,I)", F10.3)

$6 i$ CONTINUE

C

C

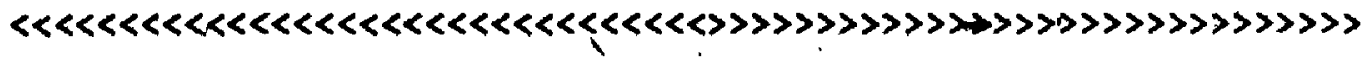

Fnd of Linearization

C

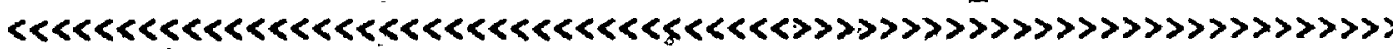

C

C

C

C

C

C

C

$* * * * * * * * * * * * * * * * * * * ; * * * * * *$

Solution of $(U, V ; W)$ using a lanted and a normal probe

Fwo equations re determined by adding $1+3,2+4$.

The third equation ts provided by eqn. of postition 5 .

$* * * * * * * * * * * * * * * * * * * * * * * * * * * * * * * * * * * * * * * * * * * * * * * * * * * * * * * * * * 1 * * *$

WRITE $(6,13)$

FORMAT (/, "MEAN VELOCITIES (3-D) USING SLANTED ONORMAL PROBE")

WRITE $(0,787)$

787 FoRuT( $/$ )

DO $346 I=1, L$

$\Lambda(1,1)=1 .+K * * 2$ 

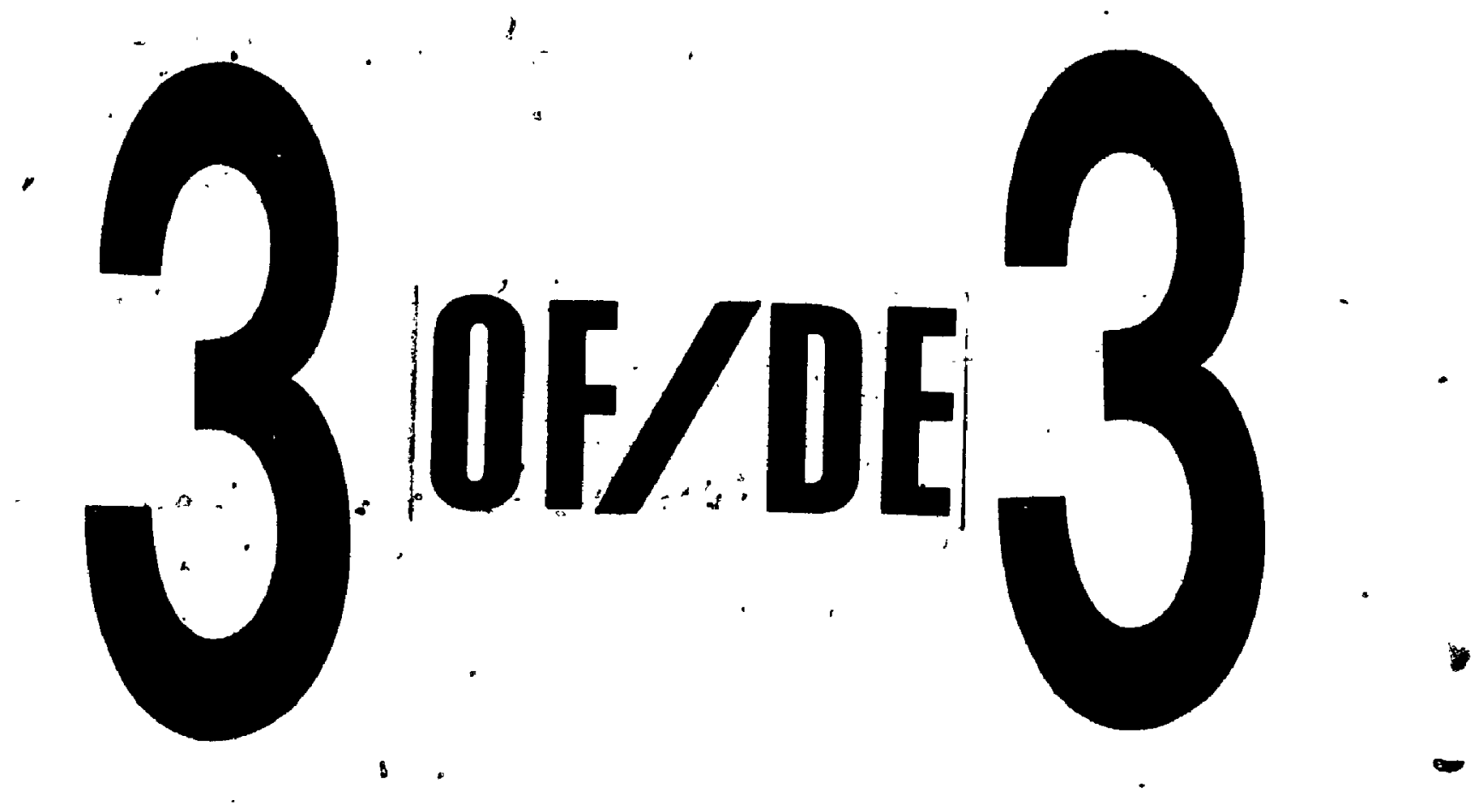

$\diamond$
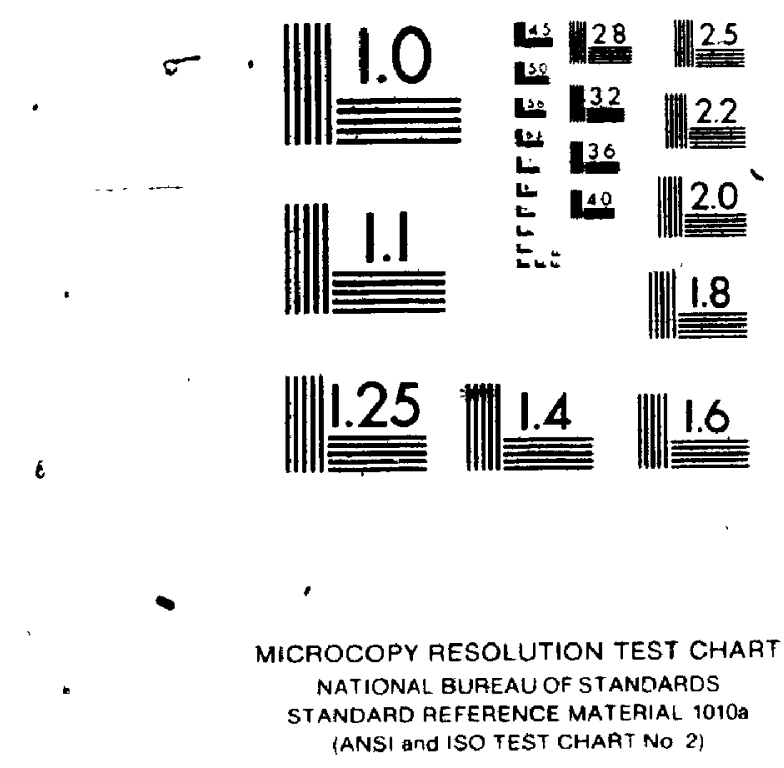

$\varepsilon$
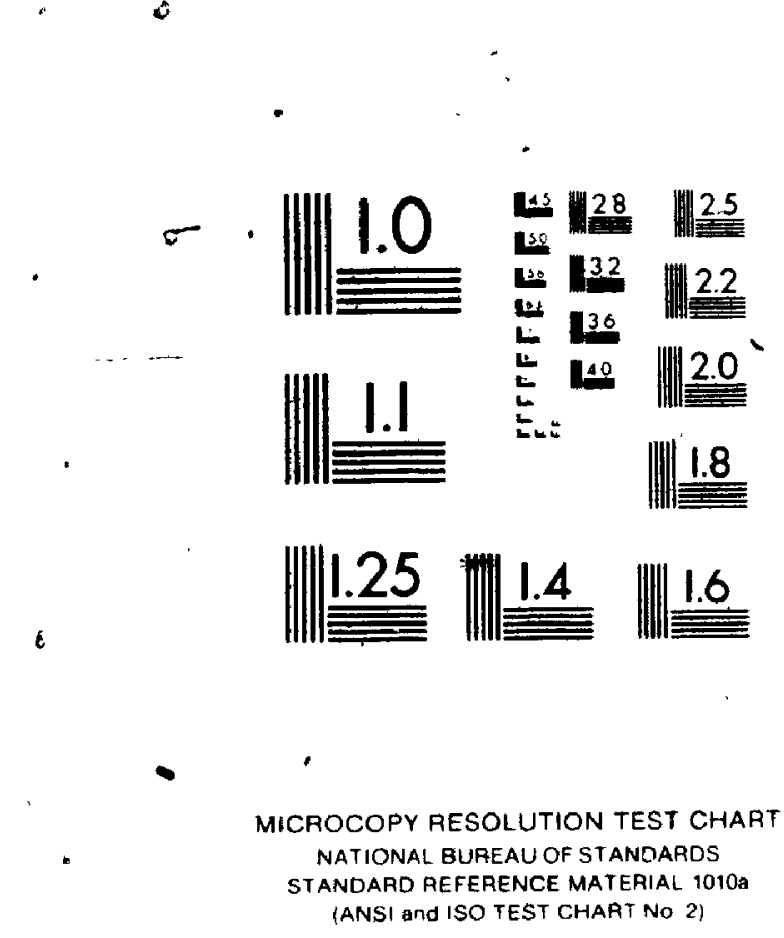


$$
\begin{aligned}
& A(1,2)=1,+K * * 2 \\
& A(1,3)=2 . *(H * * 2) \\
& A(2,1)=1 .+K * * 2 \\
& A(2,2)=2, *(B * * 2) \\
& A(2,3)=1 .+K * * 2 \\
& A(3,1)=1 .
\end{aligned}
$$$$
\Lambda(3,2)=K N * * 2
$$$$
A(3,3)=\mathrm{HN} \neq * 2
$$$$
B(1)=((E L(1, I) * * 2)+(E L(3, I) * * 2)) /(2 . * S * * 2)
$$$$
B(2)=((E L(2, I) * * 2)+(E L(4, I) * * 2)) /(2, * S * * 2)
$$$$
B(3)=(E L(5, I) * * 2) /(S N * * 2)
$$$$
\text { CALL BOLVE }
$$

$U(I)=\operatorname{SQRT}(B(1))$

$V(I)=B(2)$

$W(I)=B(3)$

WRITE $(6,12) U(I), V(I), W(I)$

12 FORMAT (2X, "U=" F10,5,4X,"VSQ=n,F10,5,4X, "WSQ=",F10.5)

345 CONTINUE .

C

C

c

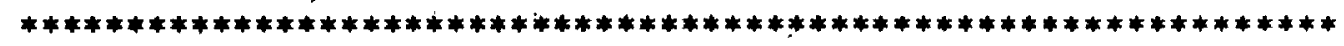

Calculation of ( $U, V)$ asing slanted probes only.

( solution of equations $1+3,2+4$ )

C

C

C

WRITE $(6,102)$

102 FORMAT (/. "MEAN VELOCITIES (2-D) USING SLANTED PROBE")

C

DO $346 I=1 . \mathrm{L}$

$A(1,1)=1 .+K * * 2$

$A(1,2)=1,+K * * 2$

$A(2,1)=1 .+K * * 2$

$A(2,2)=2, * H * * 2$

$B(1)=((E L(1, I) * * 2+(E L(3, I) * * 2)) /(S * * 2))$

$B(2)=((E L(2, I) * * 2+(E L(4, I) * * 2)) /(S * * 2))$

c

CALL SOLVE

C

$\operatorname{U2S}(I)=\operatorname{SQRT}(B(1))$

$\operatorname{V2s}(I)=B(2)$

c

WRITE(6,i03) U2S(I), V2S(I)

103 FORMAT (2X, "U2S=n,F10.6.4X,"V2S=n.F10.5)

C

346 CONTINUE 
C

C

C

c

C

C

C

ITE $(6,104)$

104

C

DO $347 \quad I=1, L$

C $(1,1)=1$

$A(1,2)=k N * * 2$

$A(2,1)=1$.

*2

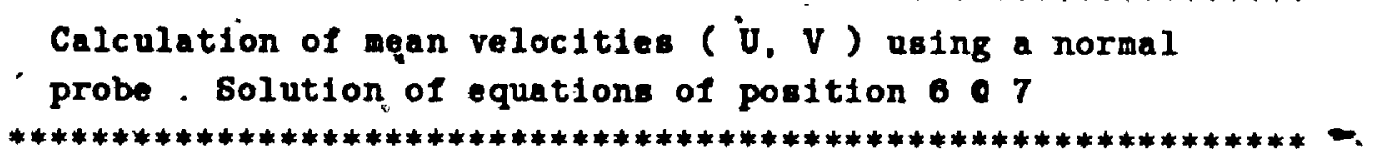

C

CALL SOLVE

C

$\mathrm{U} 2 \mathrm{~N}(\mathrm{I})=\mathrm{B}(1)$

$\operatorname{V2N}(I)=B(2)$

C

WRITE $(6,105)$ U2N (I), V2N (I)

105 FORMAT (2X, "U2N=" F10.6, 4X, "V2N=" F10.5)

C

347 CONTINUE

C

C

C

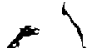

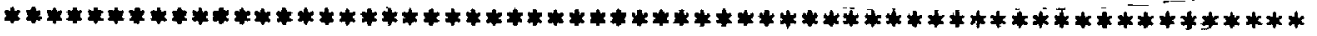

Th1s section of program calculates mean and turbulent

quantities using the $(x-v i r e)$ nethod

c

C

C

$*$

WRITE $(6,00)$

99 FORMT(/." The mean and turbulent quantíties (X-probe)") WRITE $(6,001)$

991 FORMLT(/)

WRITE $(6,101)$

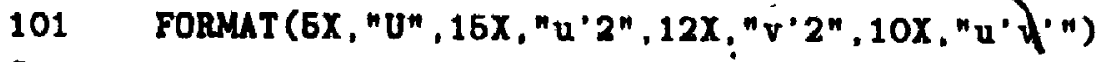

C

C

Do i1 $I=1, L$ 
C

$X X-\operatorname{EFL}(1, I) * * 2$

$X X 1=E F L(3, I) * * 2$

DEN $=4.0 *(8 * * 2)$

UVX $(I)=(X X-X X 1) / D E N$

$\operatorname{WRITE}(6,121) \mathrm{U1}(\mathrm{I}), \mathrm{US1}(\mathrm{I}), \mathrm{US2}(\mathrm{I}), \mathrm{UVX}(\mathrm{I})$

121

FORMAT (2X, F10.6,4X,F10.6,4X,F10.6,4X,F10.6)

11 CONTINUE.

C

C

C

c

C

C

C

C

14 FORMaT (/, "Calculation of turbulence assuming $(V=w=0) ")$ WRITE $(6.144)$

144 FORMAT $(/)$

DO $228 I=1, L$

UVZ $=(\operatorname{EFL}(1, I) * * 2-\operatorname{EFL}(3, I) * * 2) /(2 . *(1,-K * * 2) * S * * 2)$

$\mathrm{UWZ}=(\mathrm{EFL}(4, I) * * 2-\operatorname{EFL}(2, I) * * 2) /(2 . *(1,-K * * 2) * S * * 2)$

$\mathrm{U} 2 \mathrm{Z}=(\operatorname{EFL}(5, I) * * 2) /(\mathrm{SN} * * 2 *(1,+\mathrm{KN} * * 2))$

-O2Z=SQRT (U2Z)

ff $V 22=(1,+K * * 2) *(E F L(1, I) * * 2+\operatorname{EFL}(3, I) * * 2)$

FOL $=2 . *(1 .-K * * 2) * * 2 * S * * 2$

$\mathrm{V} 2 \mathrm{Z}=(\mathrm{V} 22 / \mathrm{FOL})-(\mathrm{EFL}(5, \mathrm{I}) * * 2 *(1+\mathrm{KN} * * 2)) /(\mathrm{SH} * * 2)$

V2Z =SQRT (V2Z)

C $\quad \mathrm{US}(\mathrm{I})=\mathrm{EL}(5, I) / 8$

WRITE $(6,8989) \mathrm{U} 2 \mathrm{Z}, \mathrm{V} 2 \mathrm{Z}, \mathrm{UVZ}, \mathrm{UWZ}$

8989 FORMAT (X, "u2=",F8.5,3X, "v2=",F8,6,3X, "w2=",F8.6,3X, nu" ${ }^{\prime}=n$

$\left.1, F 8.6,3 X, " u^{\prime} w^{\prime}=n, F 8.6\right)$

228 CONTINUE

C

C

C

C

C

C

C

16

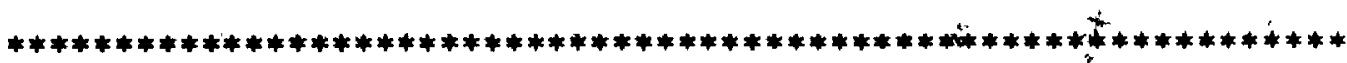

Calculation of ( $U \bullet V)$ using a slanted 'ppobe.

Equations $1+3 \cdot 2+4$ are colved by an 1teration procedure

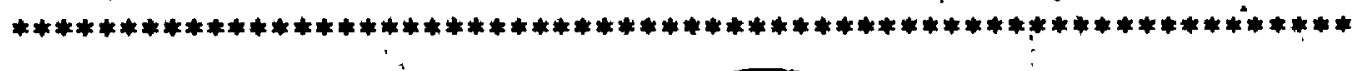

VRITE $(6,16)$

FORMAT ( ."Calculation ( $U$ $v$ ) naing BLANTED probe only") WRITE (6.151) 
FORMAT(/)

$$
\mathbf{F}=\mathbf{0 . 0}
$$

C. $\quad I=0.0$

DO $10 \mathrm{I}=1, \mathrm{~L}$

$C 1=(E L(1, I) * * 2+E L(2, I) * * 2+E L(3, I) * * 2+E L(4, I) * * 2) /(2, * S * * 2)$

C2 $.6 *(1 .+K * * 2)+H * * 2$

$C 3=(E L(2, I) * * 2+E L(4, I) * * 2) /(S * * 2)$

C $\quad C 4=(E L(6, I) * * 2 * E L(6, I) * * 2) /(2 . * S N * * 2)$

$\operatorname{VSQ}(1)=0.0$

$20 \quad \mathrm{USQ}(\mathrm{I})=(\mathrm{C} 1-\mathrm{C} 2 * \operatorname{VSQ}(\mathrm{I})) /(1 .+K * * 2)$

c

$$
\operatorname{VSq}(I)=(\mathrm{CB}-(1 .+\mathrm{K} * * 2) * \mathrm{USR}(\mathrm{I})) /(2 . * \mathrm{H} * * 2)
$$

C $\quad W S Q(I)=(C 4-U S Q(I)-.5 *(K N * * 2+H N * * 2) * V S Q(I)) /(.6 *(H N * * 2+K N * * 2))$

$\mathrm{F}=\mathrm{F}+1$

IF (F.LE. NO) GO TO 20

WRITE $(6,9990)$ USQ (I) . VSQ (I)

9990- FORMAT (2X,E12.6, 2X,E12.6)

C

C

C

10

C

C

c

c

C

C

C

IF (M. LE.NO) GO: TO 20

- $M=M+1$

CONTINUE

$* * * * * * *+1$

Solution of the turbulence quantities using full:

equations. ( $w$ is not included)

***************************4**********************************

DO $1250 \mathrm{I}=1 . \mathrm{L}$

WRITE $(6,2008)$ UE (I) , VE (I)

2008 FORMAT('U5=', F10.3.6X."V5=',F10.3)

$P A R=1 .+K * * 2$

PARN $=1$. $-K * * 2$

$P A R N N=K * 2-1$

C

QQ1 $=2 . * P A R *(U E(I) * * 2+$ VE $(I) * * 2)+4$. $*$ PARN $* U 6(I) *$ V5 (I)

$M A 1=P A R * U E(I)+P A R N * V E(I)$

$B B 1=P A R * V E(I)+P A R N * U 6(I)$

C

$Q Q 2=2 . * P A R *(U 5(I) * * 2+V 5(I) * * 2)+4 . * P A R N N * U 5(I) * V 5(I)$

$A A 2=P A R * U D(I)+P A R N N * V E$ (I)

$B B 2=P A R * V E(I)+P A R N N * U 5(I)$

C

QQ3-US $(I) * * 2+K N * * 2 * V 5(I) * * 2$

AA3-US (I) $* * 2$ 
$\mathrm{BB3}=\mathrm{KN} * * 4 * \mathrm{~V} 6(\mathrm{I}) * * 2$

$B B 4-U 6(I) * V 6(I) * K N * * 2$

$\begin{aligned} A(1.1) & =A A 1 * * 2 / Q Q 1 \\ A(1.2) & =B B 1 * * 2 / Q Q 1 \\ A(1.3) & =2 . * A 1 * B B 1 / G Q 1 \\ C \quad A(2,1) & =A \Lambda 2 * * 2 / Q Q 2 \\ A(2,2) & =B B 2 * * 2 / Q Q 2 \\ A(2.3) & =2 * A A 2 * B B 2 / Q Q 2\end{aligned}$

$A(3,1)=A$ A3/QQ3

$A(3,2)=B B 3 / Q Q 3$

$A(3,3)=B B 4 / Q Q 3$

C

C

C

c

$$
\begin{aligned}
& B(1)=(\operatorname{EFL}(1, I) * * 2) /(S * * 2) \\
& B(2)=(\operatorname{EFL}(3, I) * * 2) /(S * * 2) \\
& B(3)=(\operatorname{EFL}(5, I) * * 2) /(S N * * 2)
\end{aligned}
$$

c.

c.

c

Call solve

c.

C

WRITE(6, 565) B(1), B(2), B (3)

$665 . \operatorname{FORMAT}\left(2 X, n_{i}^{\prime} 2=n, F 10.6,2 X, n^{\prime} 2=n, F 10.6,2 X, n^{\prime} u^{\prime} v^{\prime}=n, F 10.5\right)$

1250 CQNTINUE

STOP.

END

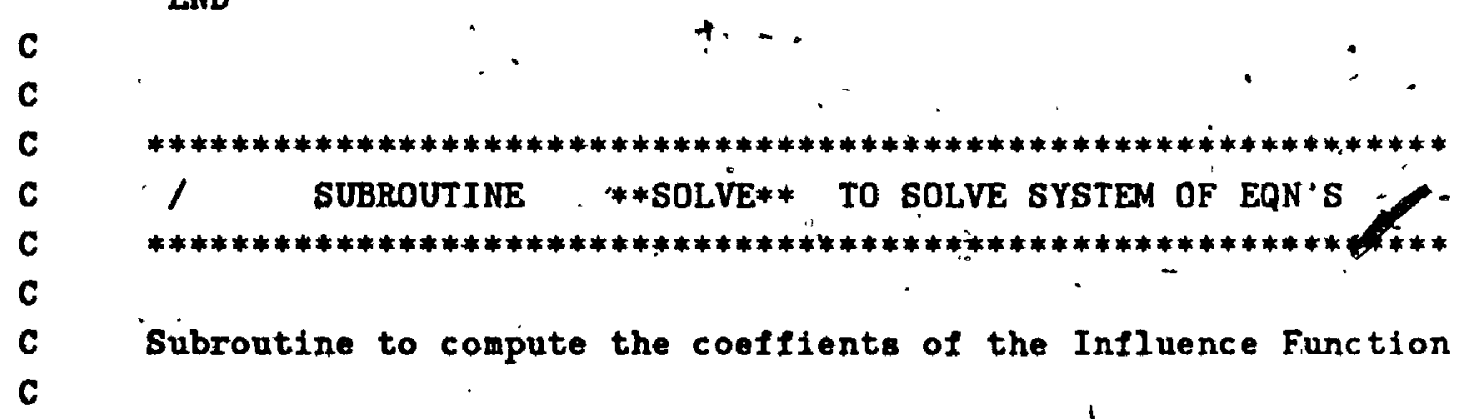

C

SUBROUTINE SOLVE

$$
\text { CoMmN } \ldots(3,3), v(3)
$$

C

C *** Search loading colum of the atrix for the pivotal element 


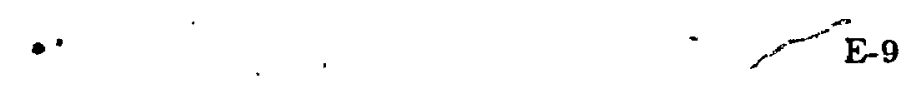

C

DO $4 K=1.2$

$I A=K$

$I B=K+1$

DO $1 I=I B, 3$

$A A=A B S(A(I A, K))$

$A B=A B S(A(I, K))$

IF (AB.GT . AA) IA=I

1 CONT INUE

C

C *** Change rows when necessary

c

IF (IA.EQ.K) GO TO 3

DO 2. $\mathrm{J}=\mathrm{K}, 3$

$\Lambda A=A(K, J)$

$\Lambda(K, J)=A(I A, J)$

$\Lambda(I A, J)=\wedge A$

2 CONT INUE

$A B=U(K)$

$U(K)=U(I A)$

$U(I A)=A B$

C

C *** Check for zero pivotal element, then proceed with forward elimination C

$3 \quad$ IF (ABS $(A(K, K))$.LE.1.OE-8) GO TO 7

DO $4 I=I B, 3$

$A A=A(I, K) / A(K, K)$

$U(I)=U(I)-\Lambda A * U(K)$

DO $4 \cdot J=I B, 3$

$\Lambda(I, J)=\Lambda(I, J)-\Lambda A * \Lambda(K, J)$

4 CONTINUE

C

' $\mathrm{C} * * *$ Solve for the unknowne by back-substitution

C.

$\operatorname{IF}(A B S(A(3,3))$. LE. $1 . O E-8)$ GO TO 7

$U(3)=U(3) / \wedge(3,3)$.

$\therefore$ DO $6 \quad x=2,3$

$I=4-K$

$A x=U(I)$

$I A=I+1$

DO $6 \mathrm{~J}=\mathrm{IA,} 3$

- $A A=A A-A(I, J) * U(J)$

5 CONTINUE

$U(I)=A \Lambda / A(I, I)$ 

C This iprogram calculates turbulence quantities using the

equations of Chapter 4 of the Thesis. In their simplified form.

This progran vas used for calculations of all mean and turbulence quantitios.

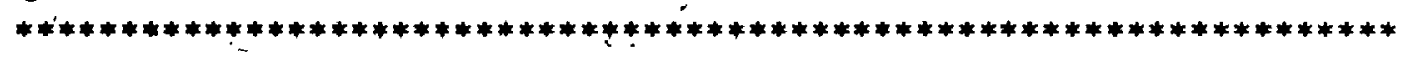

CHARACTER FNAME*64, ANS*1

CHARACTER $* 64$ FOUT

c

DIMENSION US1 (100), US2 (100), UVX (100), 'YN (100), UUO (100), YS (100)

, DIMENSION EFL $(3,100)$, USQ (100), U1 (100), EL $(3,100), 011(100)$

DIMENSION US11(100), WS (100)

REAL $\mathrm{X}$

C

PRINT*, 'DO YOU WANT TO CAECULATE (v2) component??'

PRINT*: 'IF *YES* OPTION $=0$ '

READ*;OPTION

c

PRINT*-'ENTER NUMBER OF DATA POINTS (max=100)'

READ*, $N$

PRINT*, 'ENTER SLOPE OF LINEARIZED CURVE (slanted probe)'

REND*, $S$

PRINT*. 'ENTER VALUE OF (K) CQEFFICIENT'.

READ*, K

PRINT*, Enter Max velocity $(m / B)$ '

READ* , OMAX

PRINT*, Enter Min velocity $(m / B) \cdot$

READ*, UMIN

PRINT*, 'Enter Ymax'

READ*, MAX

- PRINT*. 'Enter Ymin'

READ*, MMIN

- C

c

Read in Linearized (rms) and Mean voltiges

C position (1) refers to (psi=0, theta=45 deg)

C position (2) refers to (psi=180, thets $=46$ deg)

C

PRINT *. 'Please provide the INPUT 11le name'

READ (*,0080) FNAME

DO80 FORMTT(A) 
Opening the input eile.

$\therefore$

OPEŃ (6, FILE= FNAME, STATUS= 'OLD', ERR=090)

GO TO 000 .

909 PRINT*, Error in openning file :', FNAME

PRINT*,'File does not exist'

PRINT*, 'Do you want to onter new flle name $(Y / N)$ ?'

READ (*. ' (A)') ANS

IF (ANS .EQ.' $Y$ ' .OR. ANS .ER. ' $y^{\prime}$ ) THEN

PRINT *, 'Please provide the ISPUT file name'

READ (*, 0080) FNAME

OPEN (6, FILE=FNAME, STATUS=' OLD', ERR=099)

ELSE

PRINT*. 'I am unable to help you:" .

STOP

ENDIF

c

$990 \operatorname{READ}(5, *)(\mathrm{YN}(I), I=1, N)$

$\operatorname{READ}(5, *)(U O O(I), I=1, N)$

$\operatorname{READ}(5, *)(U S Q(I), I=1, N)$

C $\operatorname{READ}(5, *)(Y S(I), I=1, N)$

$\operatorname{READ}(5, *)(\operatorname{EFL}(1 ; I), I=1, N)$

$\operatorname{READ}(5, *)(\operatorname{EFL}(2, I), I=1, N)$

$\operatorname{READ}(5, *)(E L(2, I), I=1, N)$

C

C

C

c

C

Opening the output 1110

PRINT *, 'Do you want the output to be printed on a 11 le(Y/N)?' READ (*, $\left.(A)^{\circ}\right)$ ANS

IF ( $A N S$.EQ. 'Y' .OR' ANS .EQ. ' $y$ ') THEN'

PRINT *, 'Please provide the OUTPUT file name'

READ $(*$, OOCO) FOUT

9000 FORMAT (A)

OPEN (6, FILE=FOUT, STATUS= ' NEW' , ERR=996)

END IF

GO TO 001

006 PRINT*. 'Error in openning 111 : ' ,FOUT

PRIKT*. 'F1le alroady exists'

PRINT*. 'Do you want to overwrite 111 e (Y/N)?' 


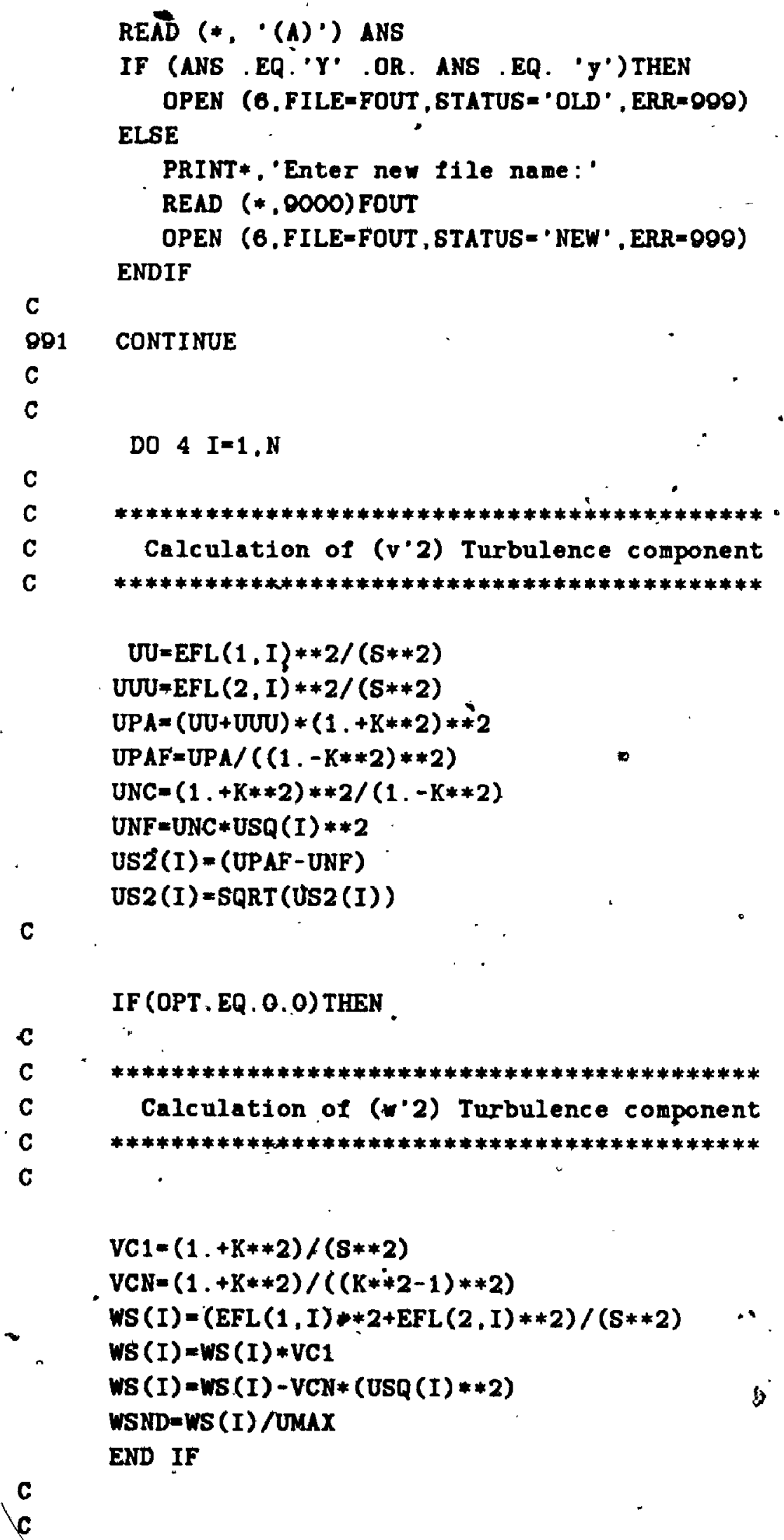

C 
c

c

C

$* * * * * * * * * * * * * * * * * * * * * * * * * * * * * * * * * * * * * * * * * * *$

Calculation of shear stresses ( $u^{\prime} v^{\prime}\left(u^{\prime} w^{\prime}\right.$ )

C

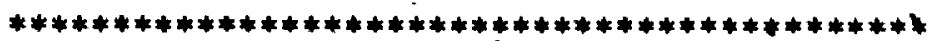

$X X=E F L(1, I) * * 2$

$X X 1=\operatorname{EFL}(2, I) * * 2$

$D E N=2.0 *(8 * * 2) *(1 .-K * * 2)$

$\mathrm{UVX}(I)=(X X-X X 1) / D E N$

C

UVX $(I)=?$ TVX $(I) *(-1$.

V2U $=U S 2(I) /(U 00(I) * 21:)$

V2UNMX $=$ US2 (I) / UMAX

C V2UMIN=US2(I)/UMIN

C

$\mathrm{U} 2 U=\mathrm{USQ}(\mathrm{I}) /(\mathrm{UUO} O(\mathrm{I}) * 21$.

U2UMAX =USQ (I)/UNAX

C U2UMIN $=U S Q(I) /$ UMIN

C

$\therefore \mathrm{C}$

- UvMax $=\operatorname{vvX}(I) /(\operatorname{mpax} * * 2) \quad \because$

UVMIN $=U V X(I) /($ UMIN**2)

C

c

C. UUMIN $=$ UUO(I)*21./UMIN

UUMAX $=$ UUO $(I) * 21$. / UMAX

c.

YNM/X $=$ YN (I)/ $\operatorname{YMAX}$

$Y M M I N=Y N(I) / Y M I L$

$Y S(I)=Y N(I)+.65$

YSMaX =YS (I)/YMaX

YSMIN $=Y S(I) /$ YMIN

C

IF (OPT ION . EQ . O) THEN

- V2UMAX-WSND

IEND IF

C

C

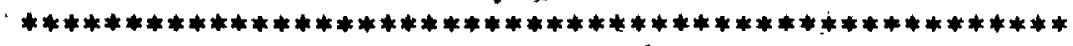

WRITE $(6,16)$ YN (I), jUO(I), U2UMaX, YS (I), V2UMaX, UVMAX

4. CONTINUE

C

16 ForMaT $(8(2 x$, F10.6))

8TOP

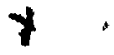

END 


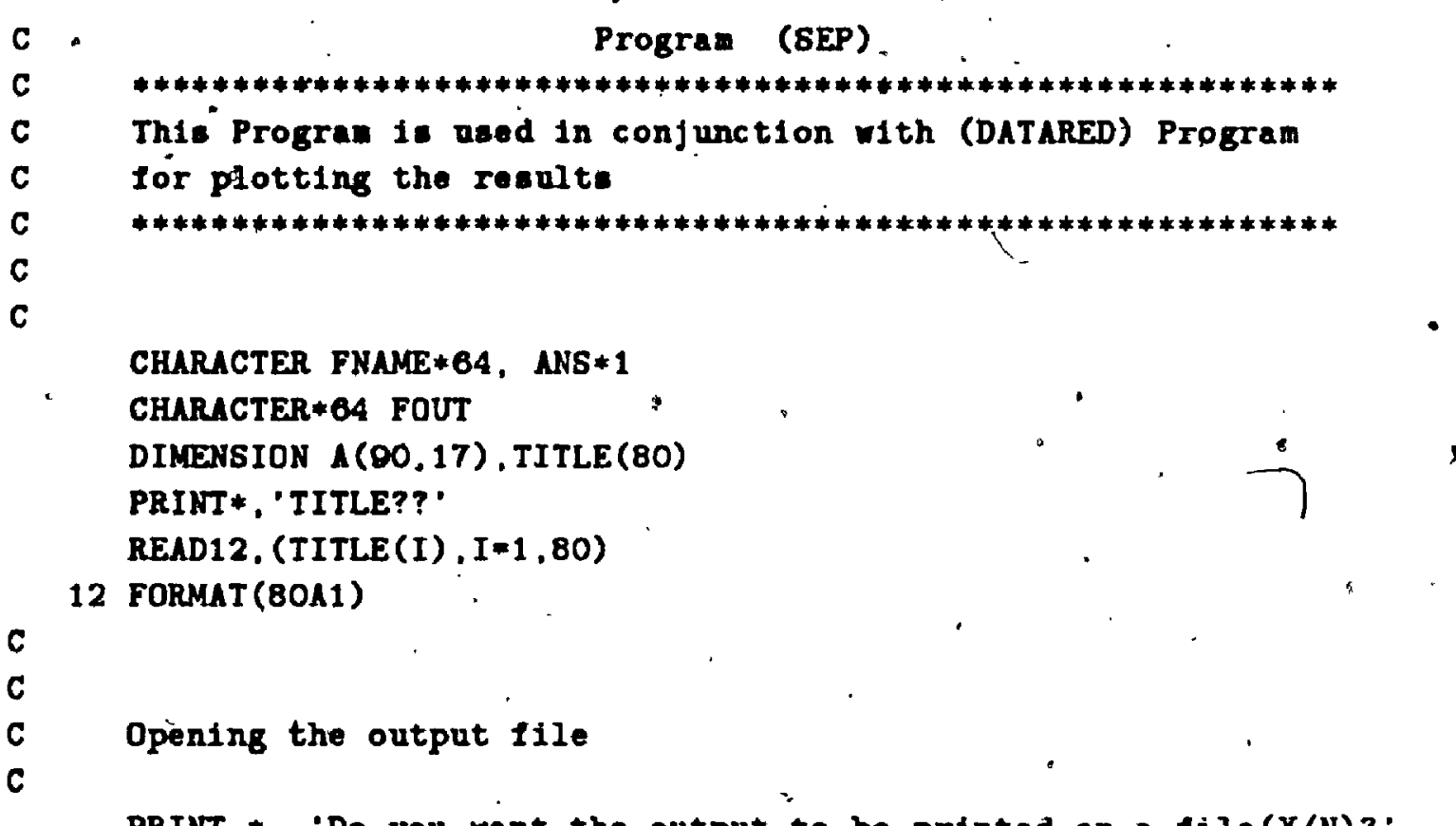

PRINT *. 'Do you want the output to be printed on a $f i l e(Y / N)$ ?' READ (*, '(A)') ANS

IF (ANS .EQ. ' $Y$ ' .OR. ANS .EQ. ' $y$ ') THEN

PRINT *. 'Please provide the OUTPUT Iile name' READ $(*, 0000)$ FOUT

9000 FORMAT (A) OPEN (6, FILE=FOUT, STATUS= ' NEW' ,ERR=996)

END IF

GO TO 091

996 PRINT*, 'Error in openning tile: ', FOUT

/PRINT*.'File already exists'

PRINT*, 'Do you want to overvrite file $(Y / N)$ ?"

READ (*, $\cdot(A) \cdot)$ ANS

IF (AYS .EQ.' $Y$ ' .OR. ANS .EQ. ' $y$ ') THEN

OPEN (6,FILE=FOUT, STATUS *'OLD', ERR=009)

ELSE

PRIYT*. 'Enter now file name:

- READ (*,0000)FOUT

OPEN (6, FIEE-FOUT, STATUS= ' NEW' , ERR=099) ENDIF

C

C

991 WRITE $(6,12)$ (TITLE (T), $I=1,80$ )

PRINT*. 'TITLE FOR $X$-AXIS??'

READ12, (TITLE (I) , $I=1,80$ )

WRITE $(0.12)(\operatorname{TITLE}(I), I=1,80)$

PRINT*. 'TITLE FOR Y-AXIsS??' 


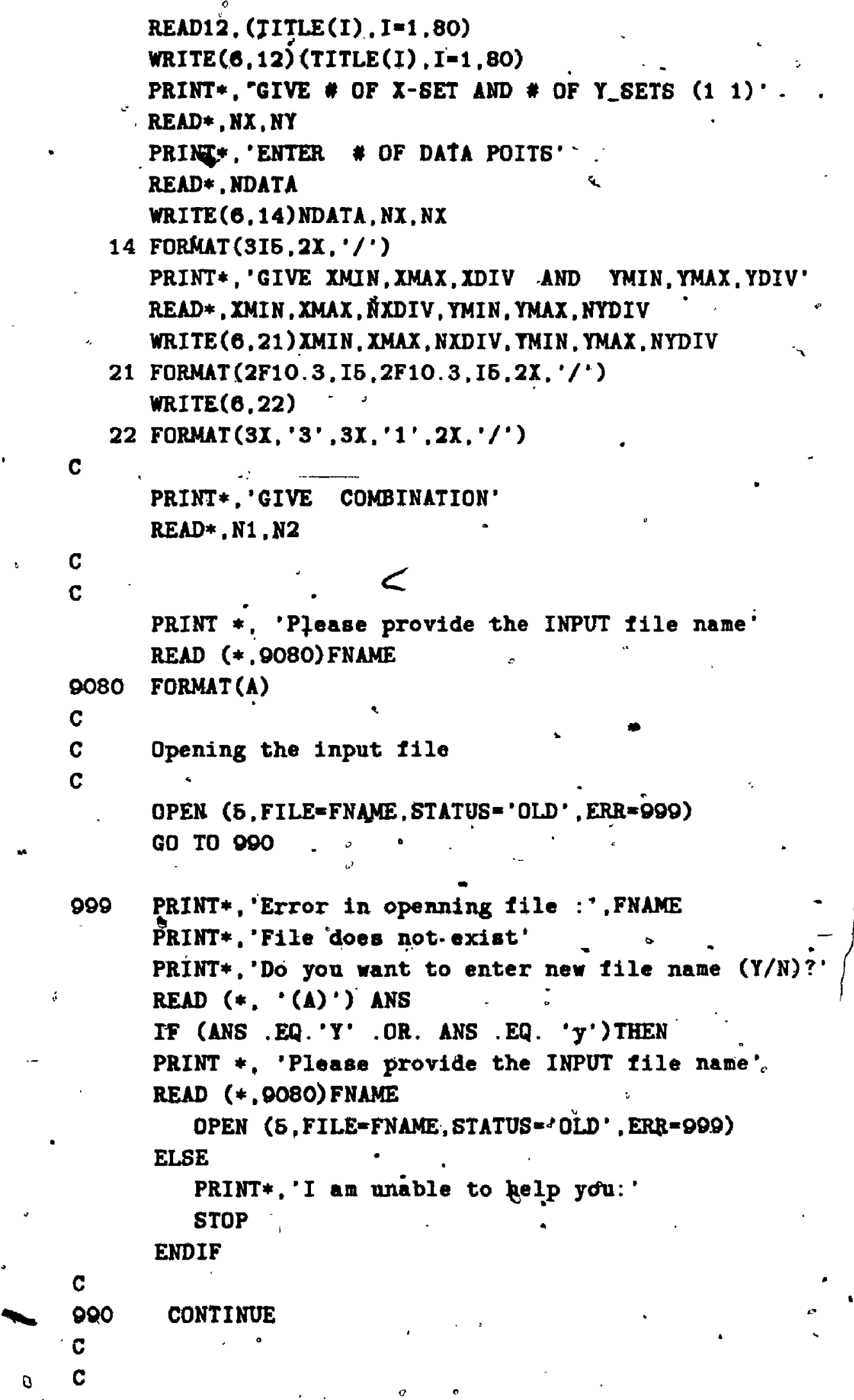


Table G.10: Results from B.L.C. Apparatus -Experiments

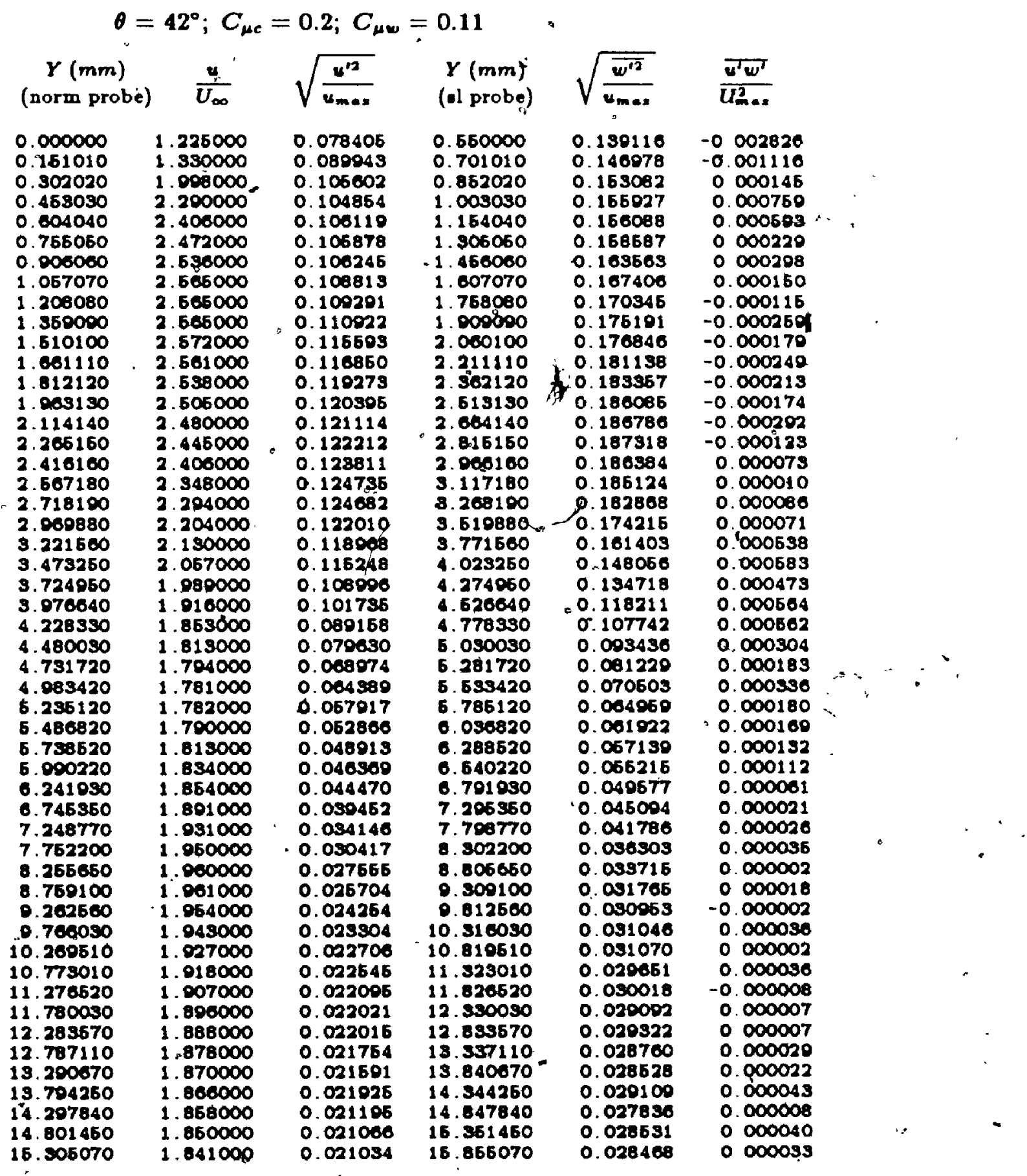




\section{Appendix F Hot-Wire Signal Analysis}

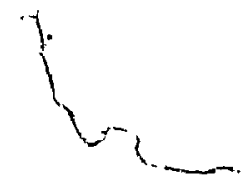

Based on the coordinate system shown in Figure 4.1 before and accordmg $1 \cdots+1 \cdots, ;, ;$ by H. Acrivlellis $[1]$, the instanteneous velocity components can be expressed . .

$$
\begin{aligned}
& u_{N}=\left(u+u^{\prime}\right) \cos \alpha+\left[\left(v+v^{\prime}\right) \cos \psi-\left(w+w^{\prime}\right) \sin \psi\right] \sin \alpha \\
& u_{T}=-\left(\dot{u}+u^{\prime}\right) \sin \alpha+\left[\left(v+v^{\prime}\right) \cos \psi-\left(w+w^{\prime}\right) \sin \psi\right] \cos \alpha \\
& u_{B}=\left(v+v^{\prime}\right) \sin \psi+\left(w+w^{\prime}\right) \cos \psi
\end{aligned}
$$

where

$u_{N}-$ normal velocity component

$u_{T}$ - tangetial velocity component

$u_{B}$ - binormal velocity component'

and

primes ( 1 ) indicate fluctuating velocity components.

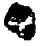

From cooling lawi33), the effective cooling velocity $u_{e f f}$ is;

$$
u_{e f f}^{2}=u_{i N}^{2}+k^{2} u_{T}^{2}+h^{2} u_{B}^{2}
$$

The linear relationship between effective cooling velocity and hot -wire ontput voltater i.

$$
E=S u_{e f f}
$$

where

$$
E-\text { output voltage }
$$

$S$ - slope of linearized plot of $E$ vs. $u_{e f f}$.

From equations 4.4 and 4.5 .

$$
u_{e f f}=\left(u_{N}^{2}+k^{2} u_{T}^{2}+h^{2} u_{B}^{2}\right)^{\frac{1}{2}}
$$




$$
\begin{aligned}
u_{e f f}= & {\left[\left\{\left(u+u^{\prime}\right) \cos \alpha+\left[\left(v+v^{b}\right) \cos \psi-\left(w+w^{\prime}\right) \sin \psi\right] \sin \alpha\right\}^{2}\right.} \\
& +k^{2}\left\{-\left(u+u^{\prime}\right) \sin \alpha+\left[\left(v+v^{\prime}\right) \cos \psi-\left(w+w^{\prime}\right) \sin \psi\right] \cos \alpha\right\}^{2} \\
& \left.+h^{2}\left\{\left(v+v^{\prime}\right) \sin \psi+\left(w+w^{\prime}\right) \cos \psi\right\}^{2}\right]^{\frac{1}{2}} \\
= & \frac{E(\psi, \alpha)}{S}
\end{aligned}
$$

After squaring both sides;

$$
\begin{aligned}
u_{e f f}^{2}= & {\left[\left\{\left(u+u^{\prime}\right) \cos \alpha+\left[\left(v+v^{\prime}\right) \cos \psi-\left(w+w^{\prime}\right) \sin \psi\right] \sin \alpha\right\}^{2}\right.} \\
& +k^{2}\left\{-\left(u+u^{\prime}\right) \sin \alpha+\left[\left(v+v^{\prime}\right) \cos \psi-\left(w+w^{\prime}\right) \sin \psi\right] \cos \alpha\right\}^{2} \\
& \left.+h^{2}\left\{\left(v+v^{\prime}\right) \sin \psi+\left(w+w^{\prime}\right) \cos \psi\right\}^{2}\right]
\end{aligned}
$$

or

$$
\begin{aligned}
\frac{E^{2}(\psi, \alpha)}{S^{2}}= & u_{e f f}^{2} \\
= & {\left[\left\{\left(u+u^{\prime}\right) \cos \alpha+\left[\left(v+v^{\prime}\right) \cos \psi-\left(w+w^{\prime}\right) \sin \psi\right] \sin \alpha\right\}^{2}\right.} \\
& +k^{2}\left\{-\left(u+u^{\prime}\right) \sin \alpha+\left[\left(v+v^{\prime}\right) \cos \psi-\left(w+w^{\prime}\right) \sin \psi\right] \cos \alpha\right\}^{2} \\
& \left.+h^{2}\left\{\left(v^{\prime}+v^{\prime}\right) \sin \psi+\left(w \cdot+w^{\prime}\right) \cos \psi\right\}^{2}\right]
\end{aligned}
$$

1

$$
\begin{aligned}
u_{e f f}^{2}= & \frac{E^{2}(\psi, \alpha)}{S^{2}} \\
= & \left(u+u^{\prime}\right)^{2} \cos ^{2} \alpha+\sin ^{2} \alpha\left[\left(v+v^{\prime}\right) \cos \psi-\left(w+w^{\prime}\right) \sin \psi\right]^{2} \\
& +2\left(u+u^{\prime}\right) \cos \alpha\left[\left(v+v^{\prime}\right) \cos \psi-\left(w+w^{\prime}\right) \sin \psi\right] \sin \alpha \\
& +k^{2}\left[\left(u+u^{\prime}\right)^{2} \sin ^{2} \alpha+\left[\left(v+v^{\prime}\right) \cos \psi-\left(w+w^{\prime}\right) \sin \psi\right]^{2} \cos ^{2} \alpha\right. \\
& \left.-2\left(u+u^{\prime}\right) \sin \alpha\left[\left(v+v^{\prime}\right) \cos \psi-\left(w+w^{\prime}\right) \sin \psi\right] \cos \alpha\right] \\
& +h^{2}\left[\left(v+v^{\prime}\right)^{2} \sin ^{2} \psi+\left(w+w^{\prime}\right)^{2} \cos ^{2} \psi+2\left(v+v^{\prime}\right)\left(w+w^{\prime}\right) \sin \psi \cos ^{2} \psi\right]
\end{aligned}
$$




$$
\frac{E^{2}(\psi, \alpha)}{S^{2}}=\left(u+u^{\prime}\right)^{2} \cos ^{2} \alpha
$$

$\approx$

$$
\begin{aligned}
& +\sin ^{2} \alpha\left[\left(v+v^{\prime}\right)^{2} \cos ^{2} \psi+\left(w+w^{\prime}\right)^{2} \sin ^{2} \psi-2\left(v+v^{\prime}\right)\left(w+w^{\prime}\right) \cos \psi \sin \psi\right] \\
& +2\left(u+u^{\prime}\right) \cos \alpha \sin \alpha\left(v+v^{\prime}\right) \cos \psi-2\left(u+u^{\prime}\right)\left(w+w^{\prime}\right) \cos \alpha \sin \alpha \sin \psi \\
+ & k^{2}\left[\left(u+u^{\prime}\right)^{2} \sin ^{2} \alpha\right. \\
+ & {\left[\left(v+v^{\prime}\right)^{2} \cos ^{2} \psi+\left(w+w^{\prime}\right)^{2} \sin \psi-2\left(v+v^{\prime}\right)\left(w+w^{\prime}\right) \sin \psi \cos \psi\right] \cos ^{2} \alpha } \\
& \left.-2\left(u+u^{\prime}\right) \sin \alpha \cos \alpha \cos \psi\left(v+v^{\prime}\right)+2\left(u+u^{\prime}\right)\left(w+w^{\prime}\right) \sin \psi \sin \alpha \cos \alpha\right] \\
+ & h^{2}\left[\left(v+v^{\prime}\right)^{2} \sin ^{2} \psi+\left(w+w^{\prime}\right)^{2} \cos ^{2} \psi+2\left(v+v^{\prime}\right)\left(w+w^{\prime}\right) \sin \psi \cos \psi\right]
\end{aligned}
$$

$$
\frac{E^{2}(\psi, \alpha)}{S^{2}}=\left(u^{2}+2 u u^{\prime}+u^{\prime 2}\right) \cos ^{2} \alpha
$$

-

$$
\begin{aligned}
& +\sin ^{2} \alpha\left[\left(v^{2}+2 v v^{\prime}+v^{\prime 2}\right) \cos ^{2} \psi+\left(w^{2}+2 w w^{\prime}+w^{\prime 2}\right) \sin ^{2} \psi\right. \\
& \left.-2\left(v w+v w^{\prime}+v^{\prime} w+v^{\prime} w^{\prime}\right) \cos \psi \sin \psi\right] .
\end{aligned}
$$$$
+2 \cos \alpha \sin \alpha \cos \psi\left(u v+u v^{\prime}+u^{\prime} v+u^{\prime} v^{\prime}\right)
$$$$
-2 \cos \alpha \sin \alpha \sin \psi\left(u w+u w^{\prime}+u^{\prime} w+u^{\prime} w^{\prime}\right)
$$$$
+k^{2}\left[\left(u^{2}+2 u u^{\prime}+u^{\prime 2}\right) \sin ^{2} \alpha+\left(v^{2}+2 v v^{\prime}+v^{\prime 2}\right) \cos ^{2} \psi \cos ^{2} \alpha\right.
$$$$
+\left(w^{2}+2 w w^{\prime}+w^{2}\right) \sin ^{2} \psi \cos ^{2} \alpha
$$$$
-2\left(v \dot{w}+v w^{\prime}+v^{\prime} w+v^{\prime} w^{\prime}\right) \cos \psi \sin \psi \cos ^{2} \alpha
$$$$
-2\left(u v+u v^{r}+u^{\prime} v+u^{\prime} v^{\prime}\right) \sin \alpha \cos \alpha \cos \psi
$$$$
\left.+2\left(u w+u w^{\prime}+u^{\prime} w+u^{\prime} w^{\prime}\right) \sin \alpha \cos \alpha \sin \psi\right]
$$$$
+h^{2}\left[\left(v^{2}+2 v v^{i}+v^{\prime 2}\right) \sin ^{2} \psi+\left(w^{2}+2 w w^{\prime}+\psi^{\prime 2}\right) \cos ^{2} \psi\right.
$$$$
\left.+2\left(v w+v w^{\prime}+v^{\prime} w+v^{\prime} w^{\prime}\right) \sin \dot{\psi} \cos \psi\right]
$$ 


$$
\begin{aligned}
& \frac{E^{2}(\psi, \alpha)}{S^{2}}=u^{2} \cos ^{2} \alpha+2 u u^{\prime} \cos ^{2} \alpha+u^{\prime 2} \cos ^{2} \alpha \\
& +v^{2} \cos ^{2} \psi \sin ^{2} \alpha+2 v v^{\prime} \cos ^{2} \psi \sin ^{2} \alpha+v^{\prime 2} \cos ^{2} \psi \sin ^{2} \alpha \\
& +w^{2} \sin ^{2} \psi \sin ^{2} \alpha+2 w w^{\prime} \sin ^{2} \psi \sin ^{2} \alpha+w^{\prime 2} \sin ^{2} \psi \sin ^{2} \alpha \\
& -2 v w \cos \psi \sin \psi \sin ^{2} \alpha-2 v w^{\prime} \cos \psi \sin \psi \sin ^{2} \alpha \\
& -2 v^{\prime} w \cos \psi \sin \psi \sin ^{2} \alpha-2 v^{\prime} w^{\prime} \cos \psi \sin \psi \sin ^{2} \alpha \\
& +2 u v \cos \alpha \sin \alpha \cos \psi+2 u v^{\prime} \cos \alpha \sin \alpha \cos \psi \\
& +2 u^{\prime} v \cos \alpha \sin \alpha \cos \psi+2 u^{\prime} v^{\prime} \cos \alpha \sin \alpha \cos \psi \\
& -2 u w \cos \alpha \sin \alpha \sin \bar{\psi}-2 u w^{\prime} \cos \alpha \sin \alpha \sin \psi \\
& -2 u^{\prime} w \cos \alpha \sin \alpha \sin \psi-2 u^{\prime} w^{\prime} \cos \alpha \sin \alpha \sin \psi \\
& +k^{2} u^{2} \sin ^{2} \alpha+2 k^{2} u u^{\prime} \sin ^{2} \alpha+k^{2} u^{\prime 2} \sin ^{2} \alpha \\
& +k^{2} v^{2} \cos ^{2} \psi \cos ^{2} \alpha+2 k^{2} v v^{\prime} \cos ^{2} \psi \cos ^{2} \alpha+k^{2} v^{2} \cos ^{2} \psi \cos ^{2} \alpha \\
& +k^{2} w^{2} \sin ^{2} \psi \cos ^{2} \alpha+2 k^{2} w w^{\prime} \sin ^{2} \psi \cos ^{2} \alpha+k^{2} w^{\prime 2} \sin ^{2} \psi \cos ^{2} \alpha \\
& -2 k^{2} v w \cos \psi \sin \psi \cos ^{2} \alpha-2 k^{2} v w^{\prime} \cos \psi \sin \psi \cos ^{2} \alpha \\
& -2 k^{2} v^{\prime} w^{\prime} \cos \psi \sin \psi \cos ^{2} \alpha-2 k^{2} u v \sin \alpha \cos \alpha \cos \psi \\
& -2 k^{2} u v^{\prime} \sin \alpha \cos \alpha \cos \psi-2 k^{2} u^{\prime} v \sin \alpha \cos \alpha \cos \psi \\
& -2 k^{2} u^{\prime} v^{\prime} \sin \alpha \cos \alpha \cos \psi+2 k^{2} u w \sin \alpha \cos \alpha \sin \psi \\
& +2 k^{2} u w^{\prime} \sin \alpha \cos \alpha \sin \psi+2 k^{2} u^{\prime} w \sin \alpha \cos \alpha \sin \psi \\
& +2 k^{2} u^{\prime} w^{\prime} \sin \alpha \cos \alpha \sin \psi \\
& +h^{2} v^{2} \sin ^{2} \psi+h^{2} v^{\prime 2} \sin ^{2} \psi+2 h^{2} v v^{\prime} \sin ^{2} \psi \\
& +h^{2} w^{2} \cos ^{2} \psi+h^{2} w^{12} \cos ^{2} \psi+2 h^{2} w w^{\prime} \cos ^{2} \psi \\
& +2 h^{2} v w \sin \psi \cos \psi+2 h^{2} v w^{\prime} \sin \psi \cos \psi \\
& +2 h^{2} w v^{\prime} \sin \psi \cos \psi+2 h^{2} v^{\prime} w^{\prime} \sin \psi \cos \psi
\end{aligned}
$$


. Noting that $2 \cos \theta \sin \theta=\sin 2 \theta$,

we have;

$$
\begin{aligned}
& \frac{E^{2}(\dot{\psi}, \alpha)}{S^{2}}=u^{2} \cos ^{2} \alpha+2 u u^{\prime} \cos ^{2} \alpha+u^{\prime 2} \cos ^{2} \alpha+u^{2} \cos ^{2} \psi \sin ^{2} \alpha \\
& +2 v v^{\prime} \cos ^{2} \psi \sin ^{2} \dot{\alpha}+v^{\prime 2} \cos ^{2} \psi \sin ^{2} \alpha+w^{2} \sin ^{2} \psi \sin ^{2} \alpha \\
& +2 w w^{\prime} \sin ^{2} \psi \sin ^{2} \alpha+w^{\prime 2} \sin ^{2} \psi \sin ^{2} \alpha-v w \sin 2 \psi \sin ^{2} \alpha \\
& -v w^{\prime} \sin 2 \psi \sin ^{2} \alpha-v^{h} w \sin 2 \psi \sin ^{2} \alpha-v^{\prime} w^{6} \sin 2 \psi \sin ^{2} \alpha \\
& +u v \sin 2 \alpha \cos \psi+u v^{\prime} \sin 2 \alpha \cos \psi+u^{\prime} v \sin 2 \alpha \cos \psi \\
& +u^{\prime} v^{\prime} \sin 2 \underline{\alpha} \cos \psi-u \omega \sin 2 \alpha \sin \psi-u w^{\prime} \sin 2 \alpha \sin \psi \\
& -u^{\prime} w \sin 2 q^{\prime} \sin \psi-u^{\prime} w^{\prime} \sin 2 \alpha \sin \psi \\
& +k^{2} u^{2} \sin ^{2} \alpha+2 k^{2} u u^{\prime} \sin ^{2} \alpha+k^{2} u^{\prime 2} \sin ^{2} \alpha \\
& +k^{2} v^{2} \cos _{i}^{2} \psi \cos ^{2} \alpha+2 k^{2} v v^{\prime} \cos ^{2} \psi \cos ^{2} \alpha+k^{2} v^{\prime 2} \cos ^{2} \psi \cos ^{2} \alpha \\
& +k^{2} w^{2} \sin \psi^{2} \cos ^{2} \alpha+2 k^{2} w w^{\prime} \sin \psi^{2} \cos ^{2} \alpha+k^{2} w^{\prime 2} \sin \psi^{2} \cos ^{2} \alpha \\
& -2 k^{2} v w \cos \psi \sin \psi \cos ^{2} \alpha-2 k^{2} v w^{\prime} \cos \psi \sin \psi \cos ^{2} \dot{\alpha} \\
& -2 k^{2} v^{\prime} w \cos \psi \sin \psi \cos ^{2} \alpha-2 k^{2} v^{\prime} w^{\prime} \cos ^{\prime} \psi \sin \psi \cos ^{2} \alpha \\
& -k^{2} u v \sin 2 \alpha \cos \psi-k^{2} u v^{\prime} \sin 2 \alpha \cos \psi \\
& -k^{2} u^{\prime} v \sin 2 \alpha \cos \psi-k^{2} u^{\prime} v^{\prime} \sin 2 \alpha \cos \psi \\
& +k^{2} u w \sin 2 \alpha \sin \psi+k^{2} u w^{\prime} \sin 2 \alpha \sin \psi_{\theta} \\
& +k^{2} u^{\prime} w^{\prime} \sin 2 \alpha \sin \dot{\psi}+k^{2} u^{\prime} w^{\prime} \sin 2 \alpha \sin \psi \\
& +h^{2} v^{2} \sin ^{2} \psi+h^{2} v^{\prime 2} \sin ^{2} \psi+2 h^{2} v v^{\prime} \sin ^{2} \psi \\
& +h^{2} w^{2} \cos ^{2} \psi+2 h^{2} w w^{\prime} \cos ^{2} \psi+h^{2} w^{\prime 2} \cos ^{2} \psi
\end{aligned}
$$

平 $h^{2} v \overline{w \sin } 2 \dot{\psi} \dot{f} \dot{h}^{2} v \bar{w}^{\prime} \operatorname{sic} 2 \psi^{-} \mp h^{2} \ddot{w}^{+} \sin 2 \psi^{-}$

$+h^{2} v^{\prime} w^{\prime} \sin 2 \psi$.

Let

$$
\begin{aligned}
& a=\cos ^{2} \alpha+k^{2} \sin ^{2} \alpha \\
& b=\sin ^{2} \alpha \cos ^{2} \psi+k^{2} \cos ^{2} \alpha \cos ^{2} \psi+h^{2} \sin ^{2} \psi \\
& c=\sin ^{2} \alpha \sin ^{2} \psi+k^{2} \cos ^{2} \alpha \sin ^{2} \psi+h^{2} \cos ^{2} \psi \\
& d=\sin 2 \alpha \cos \psi-k^{2} \sin 2 \alpha \cos \psi \\
& f=-\sin 2 \psi \sin ^{2} \alpha-k^{2} \sin 2 \psi \cos ^{2} \alpha+h^{2} \sin 2 \psi \\
& g=k^{2} \sin 2 \alpha \sin \psi-\sin 2 \alpha \sin \psi
\end{aligned}
$$


Rearranging;

$$
\begin{aligned}
\frac{E^{2}(\psi, \alpha)}{S^{2}}= & u^{2}\left[\cos ^{2} \alpha+k^{2} \sin ^{2} \alpha\right] \\
& +v^{2}\left[\cos ^{2} \psi \sin ^{2} \alpha+k^{2} \cos ^{2} \psi \cos ^{2} \alpha+h^{2} \sin ^{2} \psi\right] \\
& +w^{2}\left[\sin ^{2} \psi \sin ^{2} \alpha+k^{2} \sin ^{2} \psi \cos ^{2} \alpha+h^{2} \cos ^{2} \psi\right] \\
& +u v\left[\cos \psi \sin 2 \alpha-k^{2} \cos \psi \sin 2 \alpha\right] \\
& +v w\left[-\sin 2 \psi \sin ^{2} \alpha-k^{2} \sin 2 \psi \cos ^{2} \alpha+h^{2} \sin 2 \psi\right] \\
& +u w\left[-\sin \psi \sin 2 \alpha+k^{2} \sin \psi \sin 2 \alpha\right]
\end{aligned}
$$

or

$$
\begin{aligned}
& a u^{2}+b v^{2}+c w^{2}+d u v^{\prime \prime}+f v w^{\prime}+g u w \\
& +2 u u^{\prime} \cos ^{2} \alpha+u^{\prime 2} \cos ^{2} \alpha+2 v v^{\prime \prime} \cos ^{2} \alpha \sin ^{2} \alpha \\
& \therefore \quad+v^{\prime 2} \cos ^{2} \psi \sin ^{2} \alpha+2 w w^{\prime} \sin ^{2} \psi \sin ^{2} \alpha+w^{\prime 2} \sin ^{2} \psi \sin ^{2} \alpha \\
& \quad-v w^{\prime} \sin 2 \psi \sin ^{2} \alpha-v^{\prime} w \sin 2 \psi \sin ^{2} \alpha-v^{\prime} w^{\prime} \sin 2 \psi \sin ^{2} \alpha . \\
& \quad+u v^{\prime} \cos \psi \sin 2 \alpha+\psi^{\prime} v \cos \psi \sin 2 \alpha+u^{\prime} v^{\prime} \cos \psi \sin 2 \alpha \\
& \quad-\dot{v} w^{\prime} \sin \psi \sin 2 \alpha-u^{\prime} w \sin \psi \sin 2 \alpha-u^{\prime} w^{\prime} \sin \psi \sin 2 \alpha^{\circ} .
\end{aligned}
$$

$+2 k^{2} u u^{\prime} \sin ^{2} \dot{\alpha}+k^{2} u^{\prime 2} \sin ^{2} \alpha+2 k^{2} v v^{\prime} \cos ^{2} \psi \cos ^{2} \alpha$

$+k^{2} v^{\prime} \cos ^{2} \psi \cos ^{2} \alpha+2 k^{2} w w^{\prime} \sin ^{2} \psi \cos ^{2} \alpha+k^{2} w^{\prime 2} \sin ^{2} \psi \cos ^{2} \alpha$

$-k^{2} v w^{\prime} \sin 2 \psi \cos ^{2} \alpha-k^{2} v^{\prime} w \sin 2 \psi \cos ^{2} \alpha-k^{2} v^{\prime} w^{\prime} \sin 2 \psi \cos ^{2} \alpha$

$-k^{2} u v^{\prime} \sin 2 \alpha \cos \psi-k^{2} u^{\prime} v \sin 2 \alpha \cos \psi$

$-k^{2} u^{\prime} v^{\prime} \sin 2 \alpha \cos \psi+\hbar^{2} \operatorname{\alpha u} \omega^{\prime} \sin 2 \alpha \sin \psi$

$+k^{2} u^{\prime} w \sin 2 \alpha \sin \psi+k^{2} u^{\prime} w^{\prime} \sin 2 \alpha \sin \psi$

$+h^{2} v^{\prime 2} \sin ^{2} \psi+2 h^{2} v v^{\prime} \sin ^{2} \psi+2 h^{2} w w^{\prime} \cos ^{2} \psi$

$+h^{2} w^{\prime 2} \cos ^{2} \psi^{\prime}+h^{2} v w^{\prime} \sin 2 \psi+h^{2} v^{\prime} w \sin 2 \psi$

$+h^{2} v^{\prime} w^{\prime} \sin 2 \psi$

$=\frac{\xi^{2}(\psi, \alpha)}{S^{2}}$ 
F-7

or

$$
\begin{aligned}
& \frac{E^{2}(\psi, \alpha)}{S^{2}}=a u^{2}+b v^{2}+c w^{2}+d u v+f v w+g u w \\
& +u^{12}\left[\cos ^{2} \alpha+\mathfrak{t}^{2} \sin ^{2} \alpha\right] \ldots \\
& +v^{12}\left[\cos ^{2} \psi \sin ^{2} \alpha+k^{2} \cos ^{2} \psi \cos ^{2} \alpha+h^{2} \sin ^{2} \psi\right] \\
& +w^{\prime 2}\left[\sin ^{2} \psi \sin ^{2} \alpha+k^{2} \sin ^{2} \phi \cos ^{2} \alpha+h^{2} \cos ^{2} \psi\right] \\
& +u^{\prime} v^{\prime}\left[\cos \psi \sin 2 \alpha-k^{2} \cos \psi \sin 2 \alpha\right] \\
& \therefore+v^{i} w^{\prime}\left[-\sin 2 \psi \sin ^{2} \alpha-k^{2} \sin 2 \psi \cos ^{2} \alpha+h^{2} \sin 2 \psi\right] \\
& +u^{\prime} w^{\prime}\left[-\sin \dot{\psi} \sin 2 \alpha+k^{2} \sin \psi \sin 2 \alpha\right] \\
& +u u^{\prime}\left[2 \cos ^{2} \alpha+2 k^{2} \sin ^{2} \alpha\right] \\
& \cdots+v \varphi^{1}\left[2 \cos ^{2} \psi \sin ^{2} \alpha+2 k^{2} \cos ^{2} \psi \cos ^{2} \alpha+2 h^{2} \sin ^{2} \psi\right] \\
& +\underset{w}{w} w^{\prime}\left[2 \sin ^{2} \psi \sin ^{2} \alpha+2 k^{2} \sin ^{2} \psi \cos ^{2} \alpha+2 h^{2} \cos ^{2} \psi\right] \text {. } \\
& +v w^{\prime} \cdot\left[-\sin ^{2} 2 \psi \sin ^{2} \alpha-k^{2} \sin 2 \psi \cos ^{2} \alpha+h^{2} \sin 2 \psi\right] \text {. } \\
& +v^{\prime} w\left[-\sin 2 \psi \sin ^{2} \alpha-k \sin ^{2} 2 \psi \cos ^{2} \alpha+h^{2} \sin 2 \psi\right] \\
& +u v^{\prime}\left[\cos \psi \sin 2 \alpha-k^{2} \dot{\cos } \psi \sin 2 \alpha\right] \\
& +u^{\prime} v\left[\cos \psi \sin 2 \alpha-k^{2} \cos \psi \sin 2 \alpha\right] \text {. } \\
& +u w^{r}\left[-\sin \psi \sin 2 \alpha+k^{2} \sin \psi \sin 2 \alpha\right] \\
& +u^{\prime} w\left[-\sin \psi \sin 2 \alpha+k^{2} \sin \psi \sin 2 \alpha\right] \\
& \frac{E^{2}(\psi, \alpha)}{S^{2}}=a u^{2}+b v^{2}+c w^{2}+d u v+f v w+g u w \\
& +a u^{\prime 2}+b v^{\prime 2}+c w^{\prime 2}+d u^{\prime} v^{\prime}+f v^{\prime} w^{\prime}+g u^{\prime} w^{\prime} \\
& +2 a u u^{\prime}+2 b v v^{\prime}+2 c w w^{\prime} \\
& +d u v^{\prime}+d u^{\prime} v+f v w^{\prime}+f v^{\prime} w+g u w^{\prime}+g u^{\prime} w
\end{aligned}
$$


F-8

$$
\begin{aligned}
\frac{E^{2}(\psi, \alpha)}{S^{2}}= & a u^{2}+b v^{2}+c w^{2}+d u v+f v w+g u w \\
& +a u^{\prime 2}+b v^{\prime 2}+c w^{\prime 2}+d u^{\prime} v^{\prime}+f v^{\prime} w^{\prime}+g u^{\prime} w^{\prime} \\
& +2 a u u^{\prime}+2 b v v^{\prime}+2 c w w^{\prime} \\
& +d\left(u v^{\prime}+u^{\prime} v\right)+f\left(v w^{\prime}+v^{\prime} w\right)+g\left(u w^{\prime}+u^{\prime} w\right)
\end{aligned}
$$

Let

$$
D=2 a u u^{\prime}+2 b v v^{\prime}+2 c w w^{\prime}+d\left(u v^{\prime}+u^{\prime} v\right)+f\left(v w^{\prime}+v^{\prime} w\right)+g\left(u w^{\prime}+u^{\prime} w\right)
$$

or

$$
\begin{aligned}
v \frac{E(\psi, \alpha)}{S}= & {\left[a\left(u^{2}+u^{\prime 2}\right)+b\left(v^{2}+v^{\prime 2}\right)+c\left(w^{2}+w^{\prime 2}\right)\right.} \\
& \left.d\left(u v+u^{\prime} v^{\prime}\right)+f\left(v w+v^{\prime} w^{\prime}\right)+g\left(u w+u^{\prime} w^{\prime}\right)+D\right]^{\frac{1}{2}}
\end{aligned}
$$

Let

$$
Q=\left(a u^{2}+b v^{2}+c w^{2}+d u v+f v w+g u w\right)^{\frac{1}{2}}
$$

$=\frac{\text { Then: }}{1}$

$$
\frac{E^{2}\left(\psi_{;}^{*} \alpha\right)}{S^{2}}=\left(D+a u^{\prime 2}+b v^{\prime 2}+c w^{\prime 2}+d u^{\prime} v^{\prime}+f u^{\prime} w^{\prime}+g u^{\prime} w^{\prime}\right)+Q
$$

$$
\begin{aligned}
& \frac{E(\overline{\psi, \alpha})}{S}=\sqrt{Q^{2}+D+a u^{\prime 2}+b v^{\prime 2}+c w^{\prime 2}+d u^{\prime} v^{\prime}+f v^{\prime} w^{\prime}+g u^{\prime} w^{\prime}} \\
& =\left[Q^{2}\left(1+\frac{D}{Q^{2}}+\frac{u^{\prime 2} a+v^{\prime 2} b+w^{\prime 2} c}{Q^{2}}+\frac{u^{\prime} v^{\prime} d+v^{\prime} w^{\prime} f+u^{\prime} w^{\prime} g}{Q^{2}}\right)\right]^{\frac{1}{2}} \\
& .=Q\left[\left(1+\frac{D}{Q^{2}}+\frac{u^{\prime 2} a+v^{\prime 2} b+w^{\prime 2} c}{Q^{2}}+\frac{u^{\prime} v^{\prime} d+v^{\prime} w^{\prime} f+u^{\prime} w^{\prime} g}{Q^{2}}\right)\right]^{\frac{1}{2}}
\end{aligned}
$$


Expanding the final expression into binomial series; first degree. i.e.

$$
(1+x)^{\frac{1}{2}}=1+\frac{x}{2}
$$

Then,

$$
\frac{E(\psi, \alpha)}{S}=Q\left[\left(1+\frac{a u^{\prime 2}+b v^{\prime 2}+c w^{\prime 2}}{2 Q^{2}}+\frac{d u^{\prime} v^{\prime}+f v^{\prime} w^{\prime}+g u^{\prime} w^{\prime}}{2 Q^{2}}\right)+\frac{D}{2 Q^{2}}\right]
$$

time averaging

$$
\frac{\bar{E}(\psi, \alpha)}{S}=Q\left[\left(1+\frac{a \overline{u^{\prime 2}}+b \overline{v^{\prime 2}}+c \overline{w^{\prime 2}}}{2 Q^{2}}+\frac{d \overline{u^{\prime} v^{\prime}}+f \overline{e^{\prime} w^{\prime}}+g \overline{u^{\prime} w^{\prime}}}{2 Q^{2}}\right)\right]
$$

Note:

$D$ - drops out when expression is time averaged because it includes terms of the type $u u^{\prime}, v v^{\prime}$ (products of mean and fluctuating velocity terms),

For very small turbulence intensity $(\approx 20 \%)$, turbulent terms are very small so that they can be dropped out (smaller than time-mean velocity component).

Therefore

$$
\frac{\bar{E}^{2}(\psi, \alpha)}{S^{2}}=Q^{2}=a u^{2}+b v^{2}+c w^{2}+d u v+f v w+g u w
$$

If $(e)$ is considered to be the fluctuating component of the linearized output signal from hot-wire it can be related to the linearized instantaneous and time averaged signals, $E$ and $\bar{E}$ by;

$$
\frac{e(\psi, \alpha)}{S}=\frac{E(\psi, \alpha)}{S} \frac{-\bar{E}(\psi, \alpha)}{S}
$$

Substituting expressions of $\frac{E(\psi, \alpha)}{S}$ and $\frac{\bar{E}(\psi, \alpha)}{S}$ into the latter equation we have; 
$F+10$

$$
\begin{aligned}
\frac{e(\psi, \alpha)}{S}=Q\left[\left(1+\frac{a u^{\prime 2}+b v^{\prime 2}+c w^{\prime 2}}{2 Q^{2}}+\frac{d u^{\prime} v^{\prime}+f v^{\prime} w^{\prime}+g u^{\prime} w^{\prime}}{2 Q^{2}}\right)+\frac{D}{2 Q^{2}}\right] \\
-Q\left[\left(1+\frac{a \overline{u^{\prime 2}}+b \overline{v^{\prime 2}}+c \overline{w^{\prime 2}}}{2 Q^{2}}+\frac{d \overline{u^{\prime} v^{\prime}}+f \overline{v^{\prime} w^{\prime}}+g \overline{u^{\prime} w^{\prime}}}{2 Q^{2}}\right)\right] \\
=Q+\frac{a u^{\prime 2}}{2 Q}+\frac{b v^{\prime 2}}{2 Q}+\frac{c w^{\prime 2}}{2 Q}+\frac{d u^{\prime} v^{\prime}}{2 Q}+\frac{f v^{\prime} w^{\prime}}{2 Q}+\frac{g u^{\prime} w^{\prime}}{2 Q}+\frac{D}{2 Q} \\
\\
-Q-\frac{a \overline{u^{\prime 2}}}{2 Q}-\frac{b \overline{v^{\prime 2}}}{2 Q}-\frac{c \overline{w^{\prime 2}}}{2 Q}-\frac{d \overline{u^{\prime} v}}{2 Q}-\frac{f \overline{v^{\prime} w^{\prime}}}{2 Q}-\frac{g \overline{u^{\prime} w^{\prime}}}{2 Q}
\end{aligned}
$$

or

$$
\begin{aligned}
\frac{e(\psi, \alpha)}{S}= & \frac{1}{2 Q}\left[a\left(u^{\prime 2}-\overline{u^{\prime 2}}\right)+b\left(v^{\prime 2}-\overline{v^{\prime 2}}\right)+c\left(w^{\prime 2}-\overline{w^{\prime 2}}\right)+d\left(u^{\prime} v^{\prime}-\overrightarrow{u^{\prime} v^{\prime}}\right)\right. \\
& \left.+\dot{f}\left(v^{\prime} w^{\prime}-\overline{v^{\prime} w_{\bullet}^{\prime}}\right)+g\left(u^{\prime} w^{\prime}-\overline{u^{\prime} w^{\prime}}\right)+D\right]
\end{aligned}
$$

Let

$$
\begin{aligned}
A & =a\left(u^{\prime 2}-\overline{u^{\prime 2}}\right)+b\left(v^{\prime 2}-\overline{v^{\prime 2}}\right)+c\left(w^{\prime 2}-\overline{w^{\prime 2}}\right) \\
B & =d\left(u^{\prime} v^{\prime}-\overline{u^{\prime} v^{\prime}}\right)+f\left(v^{\prime} w^{\prime}-\overline{v^{\prime} w^{\prime}}\right)+g\left(u^{\prime} w^{\prime}-\overline{u^{\prime} w^{\prime}}\right) \\
D & =2 a u u^{\prime}+2 b v v^{\prime}+2 c w w^{\prime}+g\left(u w^{\prime}+u^{\prime} w\right)+d\left(u v^{\prime}+u^{\prime} v\right)+f\left(v w^{\prime}+v^{\prime} w\right)
\end{aligned}
$$

or

$$
\frac{e(\psi, \alpha)}{S}=\frac{1}{2 Q}[A+B+D]
$$

Squaring final equation;

$$
\{A+B+D\}^{2}=\left(A^{2}+B^{2}+D^{2}+2 A B+2 A D+2 B D\right)
$$

and

$$
\begin{aligned}
& \overline{A^{2}}=\left[\overline{a\left(u^{12}-\overline{u^{12}}\right)+b\left(v^{12}-\overline{v^{12}}\right)}+c \overline{\left(w^{12}-\overline{w^{12}}\right)}\right]^{2} \\
& =a^{2}\left(u^{\prime 2}-\overline{u^{12}}\right)^{2}+b^{2}\left(v^{\prime 2}-\overline{v^{12}}\right)^{2}+c^{2}\left(w^{\prime 2}-\overline{w^{12}}\right)^{2} \\
& =\quad+2 a b\left(u^{12}-\overline{u^{\prime 2}}\right)\left(v^{\prime 2}-\overline{v^{12}}\right)+2 a c\left(u^{\prime 2}-\overline{u^{\prime 2}}\right)\left(w^{\prime 2}-\overline{w^{12}}\right) \\
& +2 b c\left(v^{12}-\overline{v^{12}}\right)\left(w^{12}-\overline{w^{12}}\right) \\
& =a^{4}\left(u^{14}-2 u^{12} \overline{u^{12}}+\left(\overline{u^{12}}\right)^{2}\right) \cdot+b^{2}\left(v^{14}-.2 v^{12} \overline{v^{12}}+\left(\overline{v^{12}}\right)^{2}\right) \\
& +c^{2}\left(w^{14}-2 w^{12} \overline{w^{12}}+\left(\overline{w^{12}}\right)^{2}\right) \\
& +2 a b\left(u^{\prime 2} v^{\prime 2}-u^{12} \overline{v^{12}}-\overline{u^{12}} v^{\prime 2}+\overline{u^{12} v^{12}}\right) \\
& +2 a c\left(u^{\prime 2} w^{\prime 2}-u^{\prime 2} \overline{w^{12}}-\overline{u^{\prime 2}} w^{\prime 2}+\overline{u^{\prime 2} w^{12}}\right) \\
& +2 b c\left(v^{\prime 2} w^{\prime 2}-v^{12} \overline{w^{12}}-\overline{v^{12}} w^{\prime 2}+\overline{v^{\prime 2} w^{12}}\right)
\end{aligned}
$$




$$
\begin{aligned}
B^{2}= & {\left[d\left(u^{\prime} v^{\prime}-\overline{u^{\prime} v^{\prime}}\right)+f\left(v^{\prime} w^{\prime}=\overline{v^{\prime} w^{\prime}}\right)+g\left(u^{\prime} w^{\prime}-\overline{u^{\prime} w^{\prime}}\right)\right]^{2} } \\
= & d^{2}\left(u^{\prime} v^{\prime}-\overline{u^{\prime} v^{\prime}}\right)^{2}+f^{2}\left(v^{\prime} w^{\prime}-\overline{v^{\prime} w^{\prime}}\right)^{2}+g^{2}\left(u^{\prime} w^{\prime}-\overline{v^{\prime} w^{\prime}}\right)^{2} \\
& +2 d f\left(u^{\prime} v^{\prime}-\overline{u^{\prime} v^{\prime}}\right)\left(v^{\prime} w^{\prime}-\overline{v^{\prime} w^{\prime}}\right)+2 d g\left(u^{\prime} v^{\prime}-\overline{u^{\prime} v^{\prime}}\right)\left(u^{\prime} w^{\prime}-\overline{u^{\prime} w^{\prime}}\right) \\
& +2 f g\left(v^{\prime} w^{\prime}-\overline{v^{\prime} w^{\prime}}\right)\left(u^{\prime} w^{\prime}-\overline{u^{\prime} w^{\prime}}\right) \\
= & d^{2}\left(\left(u^{\prime} v^{\prime}\right)^{2}-2 u^{\prime} v^{\prime} \overline{u^{\prime} v^{\prime}}+\left(\overline{u^{\prime} v^{\prime}}\right)^{2}\right)+f^{2}\left(\left(v^{\prime} w^{\prime}\right)^{2}-2 v^{\prime} w^{\prime} \overline{v^{\prime} w^{\prime}}+\left(\overline{v^{\prime} w^{\prime}}\right)^{2}\right) \\
& +g^{2}\left(\left(u^{\prime} w^{\prime}\right)^{2}-2 u^{\prime} w^{\prime} \overline{u^{\prime} w^{\prime}}+\left(\overline{u^{\prime} w^{\prime}}\right)^{2}\right) \\
& +2 d f\left(\left(u^{\prime} v^{\prime}\right) v^{\prime} w^{\prime}-u^{\prime} v^{\prime} v^{\top} w^{\prime}-\overline{u^{\prime} v^{\prime} v^{\prime} w^{\prime}}+\overline{u^{\prime} v^{\prime} v^{\prime} w^{\prime}}\right) \\
& +2 d g\left(\left(u^{\prime} v^{\prime}\right) u^{\prime} w^{\prime}-u^{\prime} v^{\prime} \overline{u^{\prime} w^{\prime}}-\overline{u^{\prime} v^{\prime} u^{\prime} w^{\prime}}+\overline{u^{\prime} v^{\prime} u^{\prime} w^{\prime}}\right) \\
& +2 f g\left(\left(v^{\prime} w^{\prime}\right) u^{\prime} w^{\prime}-v^{\prime} w^{\prime} \overline{u^{\prime} w^{\prime}}-\overline{v^{\prime} w^{\prime} u^{\prime} w^{\prime}}+\overline{v^{\prime} w^{\prime} u^{\prime} w^{\prime}}\right)
\end{aligned}
$$

$$
\begin{aligned}
& D^{2}=\left[2 a u u^{\prime}+2 b v \dot{v}^{\prime}+2 c w w^{\prime}+g u w^{\prime}+g u^{\prime} w+d u v^{\prime}+d u^{\prime} v+f v w^{\prime}+f v^{\prime} w\right]^{2} \\
& =4 a^{2} u^{2} u^{12}+4 b^{2} v^{2} v^{12}+4 c^{2} w^{2} w^{12}+g^{2} u^{2} w^{12}+g^{2} u^{12} w^{2} \\
& +f^{2} v^{2} w^{\prime}+f^{2} v^{\prime 2}-w^{2}+d^{2} u^{2} v^{\prime 2}+\tilde{d}^{2} u^{\prime 2} v^{2} \text {. } \\
& +8 a b u v u^{\prime} v^{\prime}+8 a c u w u^{\prime} w^{\prime}+4 a g u^{2} u^{\prime} w^{\prime}+4 a g u^{\prime 2} u w \\
& +4 a f u u^{\prime} v w^{\prime}+4 a f u u^{\prime} v^{\prime} w+4 a d u^{2} u^{\prime} v^{\prime}+4 a d u v u^{\prime 2} \\
& +8 b c v w v^{\prime} w^{\prime}+4 b g v u v^{\prime} w^{\prime}+4 b g v v^{\prime} u^{\prime} w+4 b f v^{2} v^{\prime} w^{\prime} \\
& +4 b f v v^{\prime 2} w+4 b d u v v^{\prime 2}+4 b d v^{2} v^{\prime} u^{\prime}+4 c g w^{2} u^{\prime} w^{\prime} \text { 。 } \\
& +4 c g w u^{\prime} w^{\prime 2}+4 c f v w w^{\prime 2}+4 c f w^{2} w^{\prime} v^{\prime}+4 c d w w^{\prime} u v^{\prime} \\
& +4 c d w w^{\prime} u^{\prime} v^{\prime}+2 g^{2} u u^{\prime} w w^{\prime}+2 g f u w^{\prime 2} v+2 g f u w^{\prime} v^{\prime} w \\
& +2 g d u^{2} w^{\prime} v^{\prime}+2 g d u w^{\prime} u^{\prime} v+2 g f u^{\prime} v w^{\prime} w+2 g f w^{2} u^{\prime} v^{\prime} \\
& +2 g d u^{\prime} u w v+2 g d u^{\prime 2} v w+2 f^{2} w w^{\prime \prime} v v^{\prime}+2 d f u v^{\prime} v w^{\prime} \text {. } \\
& +2 d f u^{\prime} w^{\prime} v^{2}+2 d f v^{\prime 2} u w+2 d f u^{\prime} v v^{\prime} w+2 d^{2} u v^{\prime} u^{\prime} v \text {. } \\
& 2 A B=2\left[a\left(u^{12}-\overline{u^{12}}\right)+b\left(v^{12}-\overline{v^{12}}\right)+c\left(w^{\prime 2}-\overline{w^{\prime 2}}\right)\right] . \\
& \times\left[d\left(u^{\prime} v^{\prime}-\overline{u^{\prime} v^{\prime}}\right)+f\left(v^{\prime} w^{\prime}-\overline{v^{\prime} w^{\prime}}\right)+g\left(u^{\prime} w^{\prime}-\overline{u^{\prime} w^{\prime}}\right)\right] \\
& =2\left[\operatorname{ad}\left(u^{\prime 2}-\overline{u^{\prime 2}}\right)\left(u^{\prime} v^{\prime}-\overline{u^{\prime} v^{\prime}}\right)+a f\left(u^{\prime 2}-\overline{u^{\prime 2}}\right)\left(v^{\prime} w^{\prime}-\overline{v^{\prime} w^{\prime}}\right)\right.
\end{aligned}
$$




$$
\begin{aligned}
& \text {. } \\
& +a g\left(u^{\prime 2}-\overline{u^{12}}\right)\left(u^{\prime} w^{\prime}-\overline{u^{\prime} v^{\prime}}\right)+b d\left(v^{\prime 2}-\overline{v^{12}}\right)\left(u^{\prime} v^{\prime}-\overline{u^{\prime} v^{\prime}}\right) \\
& +b f\left(v^{\prime 2}-\overline{v^{\prime 2}}\right)\left(v^{\prime} w^{\prime}-\overline{v^{\prime} w^{\prime}}\right)+b g\left(v^{\prime 2}-\overline{v^{\prime 2}}\right)\left(u^{\prime} w^{\prime}-\overline{u^{\prime} w^{\prime}}\right) \\
& +c d\left(w^{\prime 2}-\overline{w^{12}}\right)\left(u^{\prime} v^{\prime}-\overline{u^{\prime} v^{\prime}}\right)+c f\left(w^{\prime 2}-\overline{w^{\prime 2}}\right)\left(v^{\prime} w^{\prime}-\overline{v^{\prime} w^{\prime}}\right) \\
& \left.+c g\left(w^{\prime 2}-\overline{w^{\prime 2}}\right)\left(u^{\prime} w^{\prime}-\overline{u^{\prime} w^{\prime}}\right)\right] \\
& \text { - } 2 A D=2\left[a\left(u^{\prime 2}-\overline{u^{\prime 2}}\right)+b\left(v^{\prime 2}-\overline{v^{\prime 2}}\right)+c\left(w^{\prime 2}-\overline{w^{\prime 2}}\right)\right] \\
& \times\left[2 a u u^{\prime}+2 b v v^{\prime}+2 c w w^{\prime}+g\left(u w^{\prime}+u^{\prime} w\right)+d\left(u v^{\prime}+u^{\prime} v\right)+f\left(v w^{\prime}+v^{\prime} w\right)\right] \\
& =2\left[2 a^{2} u u^{\prime}\left(u^{\prime 2}-\overline{u^{\prime 2}}\right)+2 a b v v^{\prime}\left(u^{\prime 2}-\overline{u^{\prime 2}}\right)\right. \\
& +2 a c w w^{\prime}\left(u^{\prime 2}-\overline{u^{12}}\right)+a g\left(u^{\prime 2}-\overline{u^{12}}\right)\left(u w^{\prime}+u^{\prime} w\right) \\
& +a f\left(u^{\prime 2}-\overline{u^{\prime 2}}\right)\left(v w^{\prime}+v^{\prime} w\right)+a d\left(u^{\prime 2}-\overline{u^{\prime 2}}\right)\left(u v^{\prime}+u^{\prime} v\right) \\
& +2 a b u u^{\prime}\left(v^{\prime 2}-\overline{v^{\prime 2}}\right)+2 b^{2} v v^{\prime}\left(v^{\prime 2}-\overline{v^{\prime 2}}\right) \\
& +2 b c w w^{\prime}\left(v^{\prime 2}-\overline{v^{12}}\right)+b g\left(v^{\prime 2}-\overline{v^{12}}\right)\left(u w^{\prime}+u^{\prime} w\right) \\
& +b f\left(v^{\prime 2}-\overline{v^{\prime 2}}\right)\left(v w^{\prime}+v^{\prime} w\right)+b d\left(v^{\prime 2}-\overline{v^{\prime 2}}\right)\left(u v^{\prime}+u^{\prime} v\right) \\
& +2 a c u u^{\prime}\left(\dot{w}^{\prime 2}-\overline{w^{12}}\right)+2 b c v v^{\prime}\left(w^{12}-\overline{w^{12}}\right) \\
& +2 c^{2} w w^{\prime}\left(w^{\prime 2}-\overline{w^{\prime 2}}\right)+c g\left(w^{\prime 2}-\overline{w^{12}}\right)\left(u w^{\prime}-u^{\prime} w\right) \\
& \left.+c f\left(w^{\prime 2}-\overline{w^{12}}\right)\left(v w^{\prime}+v^{\prime} w\right)+c d\left(w^{\prime 2}-\overline{w^{\prime 2}}\right)\left(u v^{\prime}+u^{\prime} v\right)\right] \\
& 2 B D_{*}=2\left[d\left(u^{\prime} v^{\prime}-\overline{u^{\prime} v^{\prime}}\right)+f\left(v^{\prime} w^{\prime}-\overline{v^{\prime} w^{\prime}}\right)+g\left(u^{\prime} w^{\prime}-\overline{u^{\prime} w^{\prime}}\right)\right] \\
& \times\left[2 a u u^{\prime}+2 b v v^{\prime}+2 c w w^{\prime}+g\left(u w^{\prime}+u^{\prime} w\right)+d\left(u v^{\prime}+u^{\prime} v\right)+f\left(v w^{\prime}+v^{\prime} w\right)\right] \\
& =2\left[2 a d u u^{\prime}\left(u^{\prime} v^{\prime}-\overline{u^{\prime} v}\right)+2 b d v v^{\prime}\left(u^{\prime} v^{\prime}-\overline{u^{\prime} v^{\prime}}\right)\right. \\
& +2 d c w w^{\prime}\left(u^{\prime} v^{\prime}+\overline{u^{\prime} v^{\prime}}\right)+d g\left(u^{\prime} v^{\prime}-\overline{u^{\prime} v^{\prime}}\right)\left(u w^{\prime}+u^{\prime} w\right) \\
& +d f\left(u^{\prime} v^{\prime}-\overline{u^{\prime} v^{\prime}}\right)\left(v w^{\prime}+v^{\prime} w\right)+d^{2}\left(u^{\prime} v^{\prime}-\overline{u^{\prime} v^{\prime}}\right)\left(u v^{\prime}+u^{\prime} v\right) \\
& +2 a f u u^{\prime}\left(\bar{v}^{\prime} w^{\prime}-\overline{v^{\prime} w^{\prime}}\right)+2 b f v^{\prime}\left(v^{\prime} w^{\prime}-\overline{v^{\top} w^{\prime}}\right) \\
& +2 c f w w^{\prime}\left(v^{\prime} w^{\prime}-\overline{v^{\prime} w^{\prime}}\right)+f g\left(v^{\prime} w^{\prime}-\overline{v^{\prime} w^{\prime}}\right)\left(u w^{\prime}+u^{\prime} w\right) \\
& +f^{2}\left(v^{\prime} w^{\prime}-\overline{v^{\prime} w^{\prime}}\right)\left(v w^{\prime}+v^{\prime} w\right)+f d\left(v^{\prime} w^{\prime}-\overline{v^{\prime} w^{\prime}}\right)\left(u v^{\prime}+u^{\prime} v\right) \\
& +2 a g u u^{\prime}\left(u^{\prime} w^{\prime}-\overline{u^{\prime} w^{\prime}}\right)+2 b g v v^{\prime}\left(u^{\prime} w^{\prime}-\overline{u^{\prime} w^{\prime}}\right) \\
& +2 g c w w^{\prime}\left(u^{\prime} w^{\prime}-\overline{u^{\prime} w^{\prime}}\right)+g^{2}\left(u^{\prime} w^{\prime}-\overline{u^{\prime} w^{\prime}}\right)\left(u w^{\prime}+u^{\prime} w\right) \\
& \left.+g f\left(u^{\prime} w^{\prime}-\bar{u}^{\prime} w^{\prime}\right)\left(v w^{\prime}+v^{\prime} w\right)+g d\left(u^{\prime} w^{\prime}-\overline{u^{\prime} w^{\prime}}\right)\left(u v^{\prime}+u^{\prime} v\right)\right]
\end{aligned}
$$


Therefore

$$
\frac{e^{2}(\psi, \alpha)}{S^{2}}=\frac{1}{4 Q^{2}}\left(A^{2}+B^{2}+D^{2}+2 A B+2 A D+2 B D\right)
$$

Time averaging the terms $A D$ and $B D$ will be zero becaise they contain terms of. the form $v v^{\prime}, u u^{\prime}, u w^{\prime}, w w^{\prime}$ etc. throughout which when time averaged give zero.

Therefore

$$
\frac{\overline{e^{2}}(\psi, \alpha)}{S^{2}}=\frac{1}{4 Q^{2}}\left[\overline{A^{2}}+\overline{B^{2}}+2 \overline{A B}+\overline{D^{2}}\right]
$$

Terms $\overline{A^{2}}, \overline{B^{2}}$, and $\frac{1}{2 \overline{A B}}$ contain quantities of higher order, i.e. $\overline{u^{\prime 4}}, \overline{u^{12} v^{\prime 2}}, \overline{u^{2} v^{12}}$ etc. and for low turbulence level, i.e. $\approx 20 \%$ they may be neglected.

Term $\overline{D^{2}}$ contains quantities of the order $\overline{u^{\prime 2}}, \overline{v^{\prime 2}}, \overline{u^{\prime} v^{\prime}}, \overline{v^{\prime} u^{\prime}}, \overline{v^{\prime} w^{\prime}}$ etc. and they are kept.

$$
\frac{\overline{e^{2}}(\psi, \infty)}{S^{2}}=\frac{1}{4 Q^{2}}\left(\overline{D^{2}}\right)
$$

Factoring out the $D^{2}$ term we have;

$$
\begin{aligned}
& u^{\prime 2}\left[4 a^{2} u^{2}+d^{2} v^{2}+g^{2} w^{2}+4 a g u w+4 a d u v+2 g d v w\right] \\
& +v^{\prime 2}\left[4 b^{2} v^{2}+d^{2} u^{2}+f^{2} w^{2}+4 b d u v+4 b f v w+2 d f u w\right] \\
& +w^{\prime 2}\left[4 c^{2} w^{2}+g^{2} u^{2}+f^{2} v^{2}+4 c g u w+4 c f v w+2 g f u v\right] \\
& +u^{\prime} v^{\prime}\left[8 a b u v+4 a f u w+4 a d u^{2}+4 b g v w+4 b d v^{2}+2 g f w^{2}+2 g d u w+2 d f v w+2 d^{2} u v\right] \\
& +u^{\prime} w^{\prime}\left[8 a c u w+4 a g u^{2}+4 a f u v+4 c g w^{2}+4 c d v w+2 g^{2} u w+2 g d u v+2 g f v w+2 d f v^{2}\right] \\
& +v^{\prime} w^{\prime}\left[8 b c v w+4 b g v u+4 b f v^{2}+4 c f w^{2}+4 c d u w+2 g f u w+2 g d u^{2}+2 f^{2} v w+2 d f u v\right]
\end{aligned}
$$

$D^{2}$ can be expressed as;

$$
D^{2}=\left(a_{1} u^{\prime}+b_{1} v^{\prime}+c_{1} w^{\prime}\right)^{2}
$$

where

$$
\begin{aligned}
a_{1} & =2 a u+d v+g w \\
b_{1} & =2 b v+f w+d u \\
c_{1} & =2 c w+g u+f v
\end{aligned}
$$




$$
\begin{aligned}
u e f f= & {\left[\left\{\left(u+u^{\prime}\right) \cos \alpha+\left[\left(v+v^{b}\right) \cos \psi-\left(w+w^{\prime}\right) \sin \psi\right] \sin \alpha\right\}^{2}\right.} \\
& +k^{2}\left\{-\left(u+u^{\prime}\right) \sin \alpha-\left[\left(v+v^{\prime}\right) \cos \psi-\left(w+w^{\prime}\right) \sin \psi\right] \cos \alpha\right\}^{2} \\
& \left.+h^{2}\left\{\left(v+v^{\prime}\right) \sin \psi-\left(w+w^{\prime}\right) \cos \psi\right\}^{2}\right]^{\frac{1}{2}} \\
= & \frac{E(\psi, \alpha)}{S}
\end{aligned}
$$

After squaring both sides;

$$
\begin{aligned}
u_{e f f}^{2}= & {\left[\left\{\left(u+u^{\prime}\right) \cos \alpha+\left[\left(v+v^{\prime}\right) \cos \psi-\left(w+w^{\prime}\right) \sin \psi\right] \sin \alpha\right\}^{2}\right.} \\
& +k^{2}\left\{-\left(u+u^{\prime}\right) \sin \alpha+\left[\left(v+v^{\prime}\right) \cos \psi-\left(w+w^{\prime}\right) \sin \psi\right] \cos \alpha\right\}^{2} \\
& \left.+h^{2}\left\{\left(v+v^{\prime}\right) \sin \psi+\left(w+w^{\prime}\right) \cos \psi\right\}^{2}\right]
\end{aligned}
$$

$$
\begin{aligned}
\frac{E^{2}(\psi, \alpha)}{S^{2}}= & u_{e f f}^{2} \\
= & {\left[\left\{\left(u+u^{\prime}\right) \cos \alpha+\left[\left(v+v^{\prime}\right) \cos \psi-\left(w+w^{\prime}\right) \sin \psi\right] \sin \alpha\right\}^{2}\right.} \\
& +k^{2}\left\{-\left(u+u^{\prime}\right) \sin \alpha-\left[\left(v+v^{\prime}\right) \cos \psi-\left(u+w^{\prime}\right) \sin \psi\right] \cos \alpha\right\}^{2} \\
& \left.-h^{2}\left\{\left(v^{\prime}+v^{\prime}\right) \sin \psi+\left(w_{\diamond}+w^{\prime}\right) \cos \psi\right\}^{2}\right]
\end{aligned}
$$

3

$$
\begin{aligned}
u_{e f f}^{2}= & \frac{E^{2}(\psi, \alpha)}{S^{2}} \\
= & \left(u+u^{\prime}\right)^{2} \cos ^{2} \alpha+\sin ^{2} \alpha\left[\left(v+v^{\prime}\right) \cos \psi-\left(w+w^{\prime}\right) \sin \psi\right]^{2} \\
& +2\left(u+u^{\prime}\right) \cos \alpha\left[\left(v+v^{\prime}\right) \cos \psi-\left(w+w^{\prime}\right) \sin \psi\right] \sin \alpha \\
& +k^{2}\left[\left(u+u^{\prime}\right)^{2} \sin ^{2} \alpha+\left[\left(v+v^{\prime}\right) \cos \psi-\left(w+w^{\prime}\right) \sin \psi\right]^{2} \cos ^{2} \alpha\right. \\
& \left.-2\left(u+\dot{u}^{\prime}\right) \sin \alpha\left[\left(v+v^{\prime}\right)^{\prime} \cos \psi-\left(w+w^{\prime}\right) \sin \psi\right] \cos \alpha\right] \\
& +h^{2}\left[\left(v+v^{\prime}\right)^{2} \sin ^{2} \psi+\left(w+w^{\prime}\right)^{2} \cos ^{2} \psi+2\left(v+v^{\prime}\right)\left(w+w^{\prime}\right) \sin \psi \cos \psi\right]
\end{aligned}
$$


$(3-1$

APPENDIX G

TABULATED RESULTS

B.L.C. Apparatus

$\therefore$

9

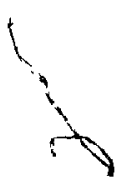

? 
Táble G.1: Results from B.L.C. Apparatus Experiments"

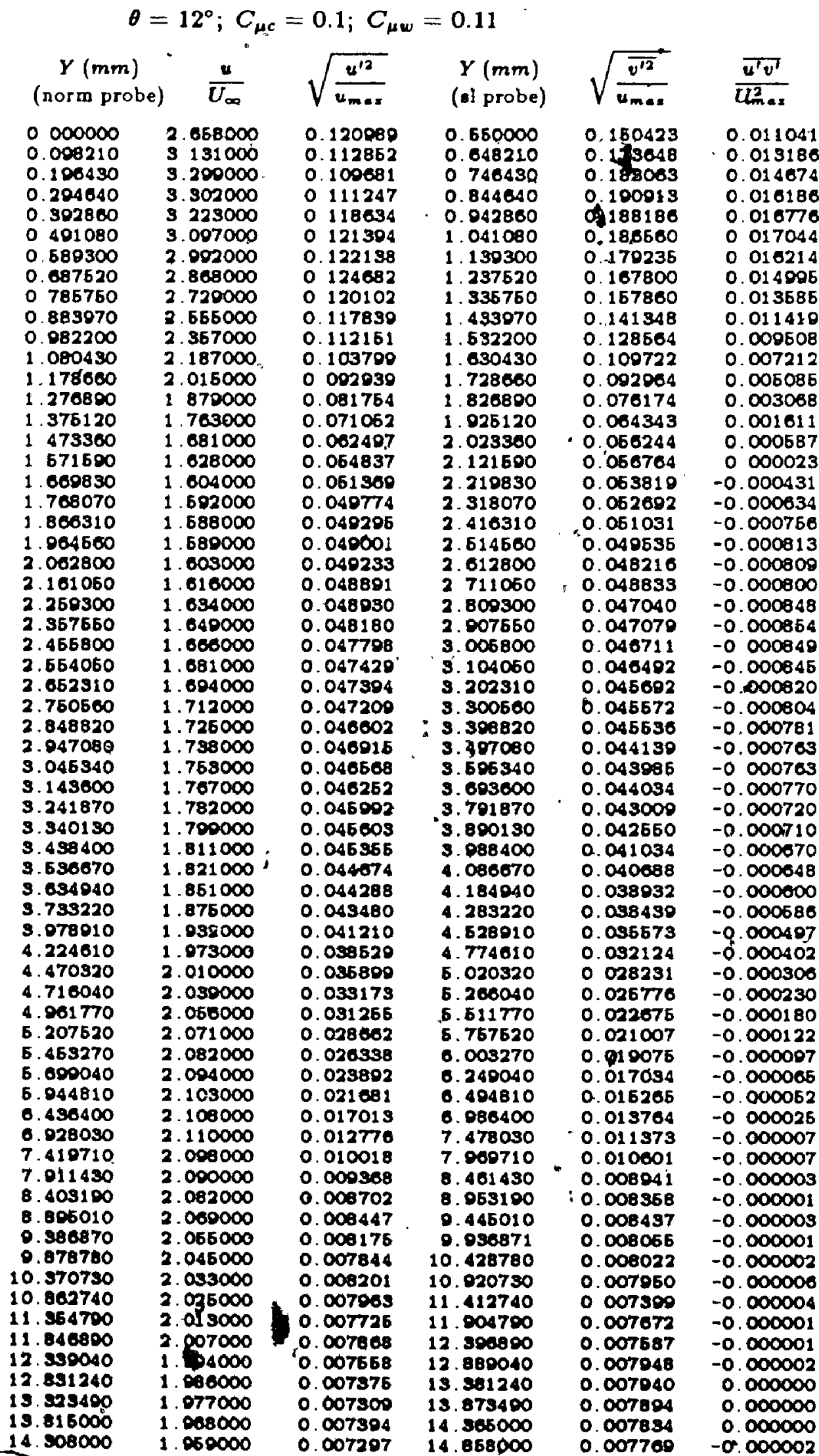


1

Table G.2: Results from B.L.C. Apparatue Experiments

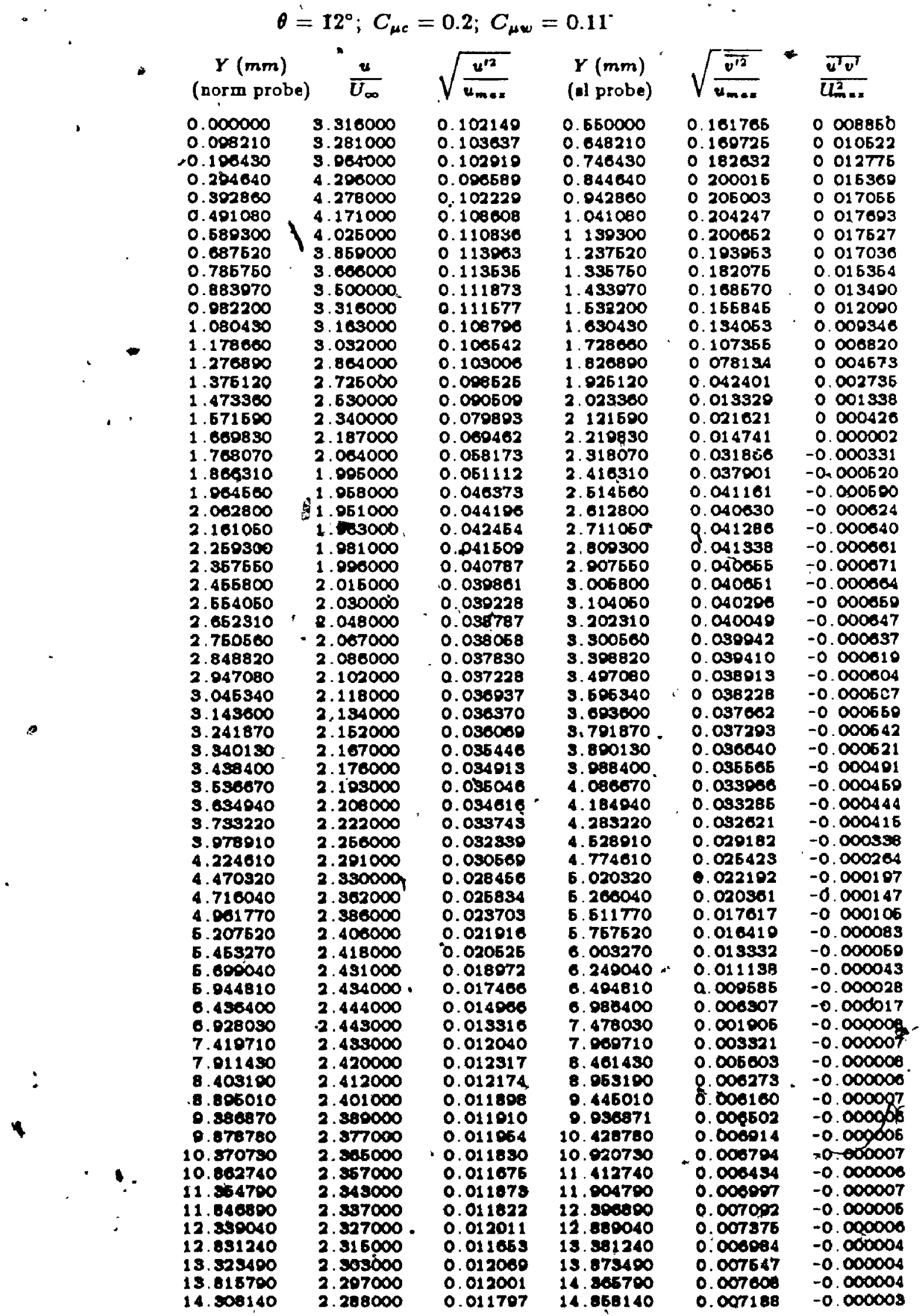


Table G.3: Results from B.L.C. Apparatus Experiments

$$
\theta={ }^{\prime} 12^{\circ} ; C_{\mu c}=0.22 ; C_{\mu \omega}=0.11
$$

$Y$ ( $\mathrm{mm}$ )
(norm probe)

0.000000

0.098210

0.190430

0.294640

0.302860

0.491080

- 0.689300

0.687520

0.785760

0.883970

0.082200

$r \begin{array}{r}0.982200 \\ 1.1 \times 804000 \\ 1.1 \% 6000\end{array}$

1.276000

1.376120

1.473360

1.671600

1.669830

1.768070

1.860310

1. .064560

2.062800

2. 161050

2. 260300

2. $\$ 67560$

2. 465800

2. 654060

2. 652310

2.760660

2.848820

2.947080

3.046340

\$. 143600

3.241870

3.3401 50

3. 438400

3. 536670

3. 634040

3.735220

3. 078010

4.224610

4. 470320

4.716040

4.061770

6. 207620

6. 453270

6. 600040

5.044810

6. 450400

- . 028080

7.410710

7.011430

8. 409100

8. 805010

- 0.386870

9.878780

10. 370750

10.862740

11.864700

11.846890

12. 350040

12. 831240

13.323490

13.815700

. 14.808140

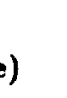

\section{$\frac{u}{U_{\infty}}$}

3. 200000 .

3. 347000

4.080000

4.626000

4. 634000

4.430000

4.278000

4.088000

3. 000000

3. 721000

3. 628000

3. 367000

3. 216000

3. 050000

2. 864000

2.656000

2.444000

2.272000

2. 130000

2.047000

1.005000

1.000000

1.994000

2. 000000

2.020000

2.038000 .

2. 080000

2.074000

2. 027000

2. 118000

2. 136000

2. 163000

2. 168000

2. 184000

2. 200000

2. 213000

2.223000

2. 243000

2. 262000

2.297000

2. 335000

2. 373000

2. 410000

2.430000

2. 456000

2. 470000

2. 482000

2.401000

2. 601000

2. 800000

2.401000

2. 478000

2.464000

2.465000

2.448000

2.480000

2.410000

2. 404000

2. 300000

2. 386000

2. 377000

2. 304000

2. 357000

2. 342000

2. 356000

$$
\sqrt{\frac{u^{12}}{u_{\max }}}
$$

o 116047

o. 100032

o. 108100

0. 102600

0.11068

0.113683

0.116666

0.110060

0.120388

0.118415

0.118304

0.114415

0.111680

0.107932

0.103330

0.004217

0.083126

0.071857

0.001112

0.052056

0.046411

0.043864

0.041864

0.040708

0.039349

0.038801

0.038515

0.037400

0.038027

0.038612

0.030103

0.035685

0.035170

0.034804

0.034604

0.034182

0.039731

0.039126

C.032050

0.031353

0.020216

0.028821

0.024688

0.022465

0.020178

0.018887

0.017160

0.015563

0.015412

0.012142

0.000437

0.007723

0.007167

0.006740

0.006762

0.006052

$0.00640 \%$

0.000378

0.006567

0.000510

0.006473

0.006178

0.000034

0.000221

0.006281
$Y(\mathrm{~mm})$

(al probe)

0.550000

0.648210

o. 746430

0.844640

0.942860

1.041080

1. 190300

1. 237620

1. 335760

1. 433970

1. 532200

1. 080400

1. 728600

1.826890

1.025120

2.023360

2. 121600

2. 210830

2. 318070

2.410310

2.614560

2. 612800

2. 711060

2. 800300

2. 007550

3. 005800

3. 104050

3. 202310

3. 300580

3. 308820

3. 497080

3. 696340

3. 603000

3. 791870

3.800130

3. 088400

4.086070

4. 184940

4. 263220

4.528010

4. 774610

6. 020320

6. 268040

6. 611770

5.767620

6. 003270

6. 240040

0.404810

6.986400

7.478090

7. 900710

8.461430

8. 053100

0.446010

- 08587i

10.428780

10. 020750

11. 412740

11.004700

12.306800

12. 880040

13.381240

18.873400

14.865700

14.058140

$\sqrt{\frac{\overline{v^{\prime 2}}}{u_{\max }}}$

$\frac{\overline{u^{\prime} v^{\prime}}}{\overline{U_{\max }^{2}}}$

$0.165420 \quad 0000049$

$0.176563 \quad 0.012005$

0.188462

0.108142

0.107571

0.104012

0.185650

0.173240

0.150360

0.146860

0.130083

0.110000

0.080276 


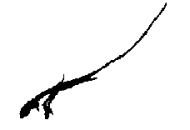

Table G.4: Results from B.L.C. Apparatus Experiments

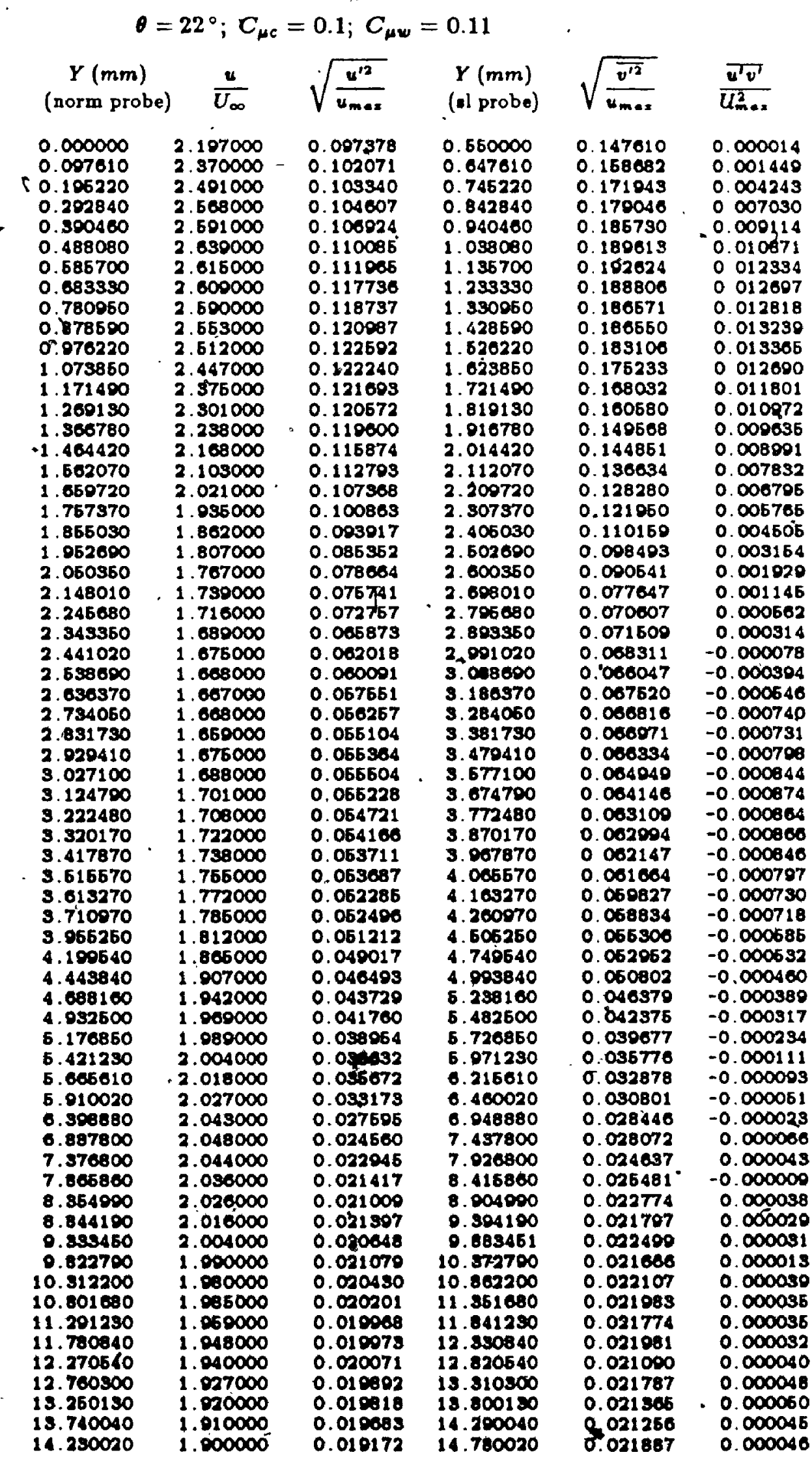


Table G.5: Resulta from B.L.C. Apparatus Experiments

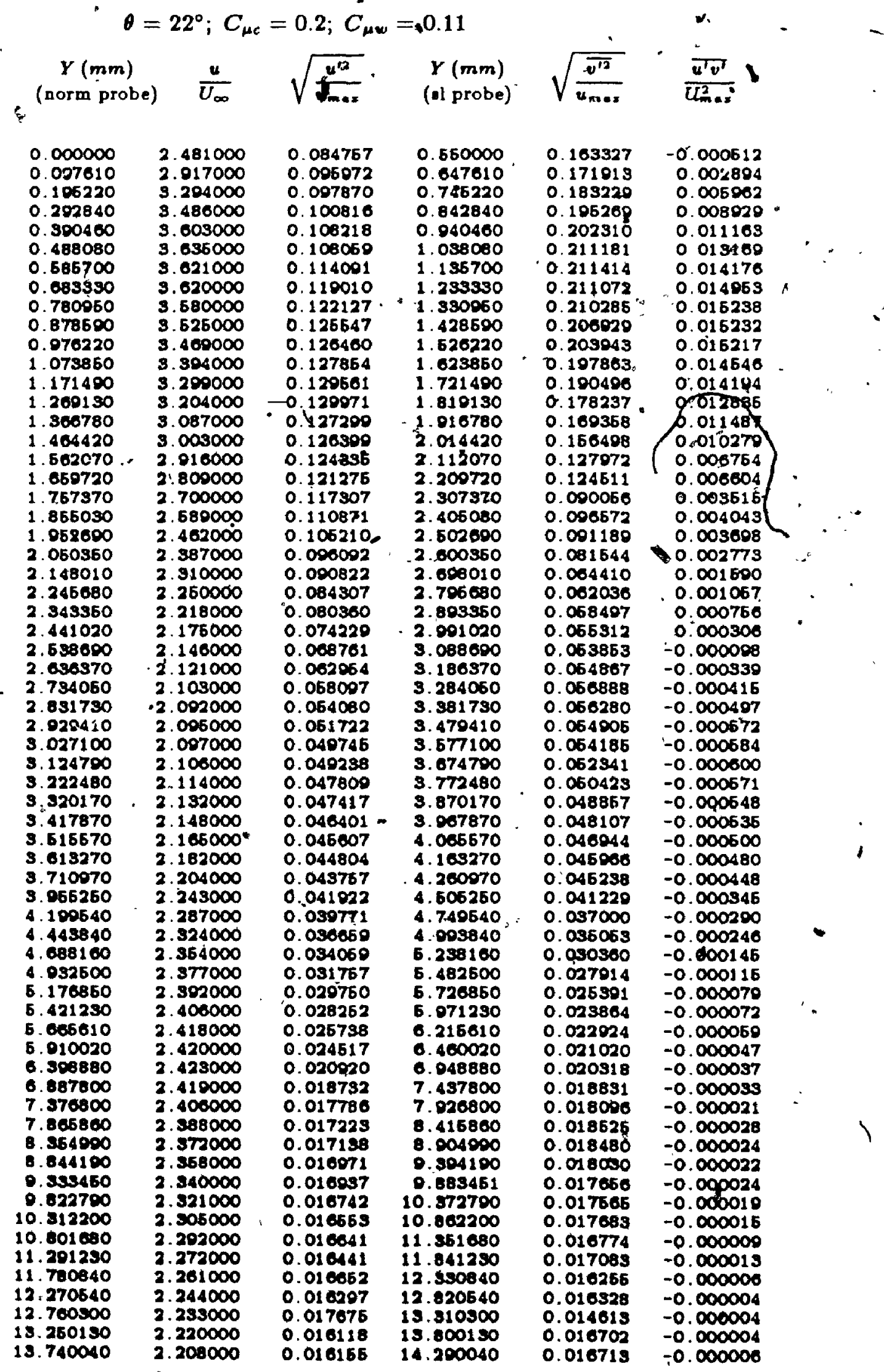


Table G.6: Pesults from B.L.C. Apparatus Experiments

\begin{tabular}{|c|c|c|c|c|}
\hline $\begin{array}{c}\dot{Y}_{4}(\mathrm{~mm}) \\
\text { (normprobe) }\end{array}$ & $\frac{u}{U_{\infty}}$ & $\frac{u^{\prime 2}}{u_{\max }}$ & $\begin{array}{c}Y(\mathrm{~mm}) \\
\text { (al probe) }\end{array}$ & $\frac{\overline{v^{12}}}{u_{m-x}}$ \\
\hline
\end{tabular}

$+$

0.000000

0.097610

0.108220

0.292840

0.390400

0.488006

0.685700

0.683330

0.780050

0.878590

0.976220

1.073850

1.171400

1. 260130

1. 360780

1. 464420

1. 662070

1. 659720

1.757370

1. 865030

1. 062600

2. 050350

$+2.148010$

2.246680

2. 343350

- 2.441020

2. 538000

2. 630370

2.734060

2.831730

2.220410

3.027100

3.124700

3.222480

3 920170 .

3.417870

3.615670

3.613270

3.710070

3. 065260

4. 100540 .

4 ? 43840 .

4.688160

4.932500

b. 176850

5. 421230

5.065610

5. 910020

.6. $3988 B 0$

6. 887800

7. 376800

7. 865600

8.854900

- 8.844100

0.356450

0.822700

10. 312200

10.801680

11.201200

11. 780840

12. 270600

12. 760500

13. 250130

13.740040
2.746000

3. 426000

3.734000

3.831000

3.873000

3. 843000

3.788000

3,710000

3. 026000

3. 600000

3. 421000

3. 328000

f. 238000

S. 165000

3. 005000

2. 076000

2.803000

2.776000

2. 800000

2. 504000

2. 520000

2. 443000

2. 377000

2.309000

2.257000

2.225000

2. 103000

2. 178000

2.174000

2. 180000 .

2. 103000

2.202000

2.214000

2.230000

2.248000

2.260000

2.289000

2.204000

2. $\$ 12000$

2.345000

2. $\$ 76000$

2. 405000

2. 438000

2.453000

2. 475000

2. 400000

2.498000

2. 502000

2. 511000

2. 502000

2.491000

2. 479000

2. 480000

2. 444000

2.425000

2.410000

2. 004000

2. 383000 .

2. 300000

2.864000

2.357000

2. 330000

2. 311000

T. $\$ 01000$

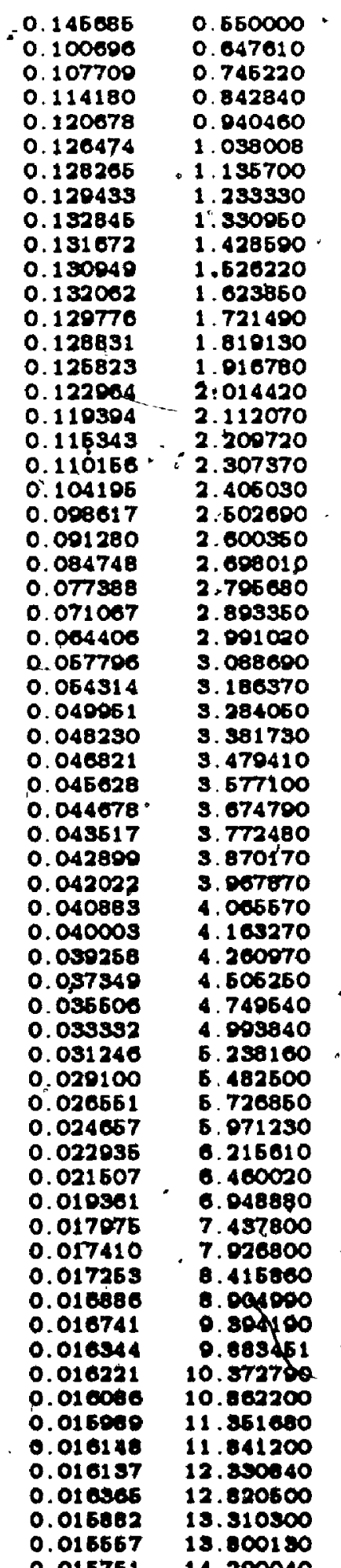

0.145085

o. 107643

0. 168000

0. 177070

0.183201

- 186300

0.188088

0.101208

- 102000

0.187774

0.186808

0.184027

0.177476

0.179016

0.160020

0. 149862

0.141267

0.132510

0. 118465

0. 100005

- 0.007497

0.086270

0.073423

0.084553

0.069428

0.058231

0.067410

0.058002 .

0.067100

0.066871

0.065601

0.062566

0.050836

0.040681

0.048172

0.046108

0.046710

0.044903

0.043652

0.042300

0.030201

$0: 036246$

0.032863

0.027482

0. 025032

0.023002

0.022840

0.021422

0. 020473

0.010414

0. 017501

0.017605

0.017245

0.017600

0.017161

0.016020

0.016786

0.016088

0.010004

0.016442

0.016107

0.015840

0.018220

0.016003

o. 016208
0.002743

0 006160

0. 007601

0.010020

0011296

0.012420

0.013421.

0013906

0.013003

0. 013782

0.013422

0.012716

0.012120

0.010004

0.000756

0.008804

0.007807

0. 006310

0.005400

0.004277

0.003200

0.002163

0.001310

- 000700

- 000264

$-0.000045$

$-0.000250$

$-0.000422$

$-0.000471$

$-0.000625$

$-0.000565$

$-0.000523$

$-0.000623$

$-0.000480$

$-0.000470$

$-0.000462$

$-0.000430$

$-0.000413$

$-0.000373$

- 0000304

0.000226

- 0000180

$-0.000106$

$-0.000076$

$-0.000048$

$-0.000046$

$-0.000034$

$-0.000027$

$-0.000026$

$-0.000022$

$-0.000024$

$-0.000021$

$-0.000010$

$-0.000018$

$-0.000015$

$-0.000010$

$-0.00000$

$-0.000005$

0.000000 .

$-0.000006$

$-0.000007$

$-0.000004$

$-0.000002$

0.000004 
Table G.7: Results from B.L.C. Apparatus Experiments

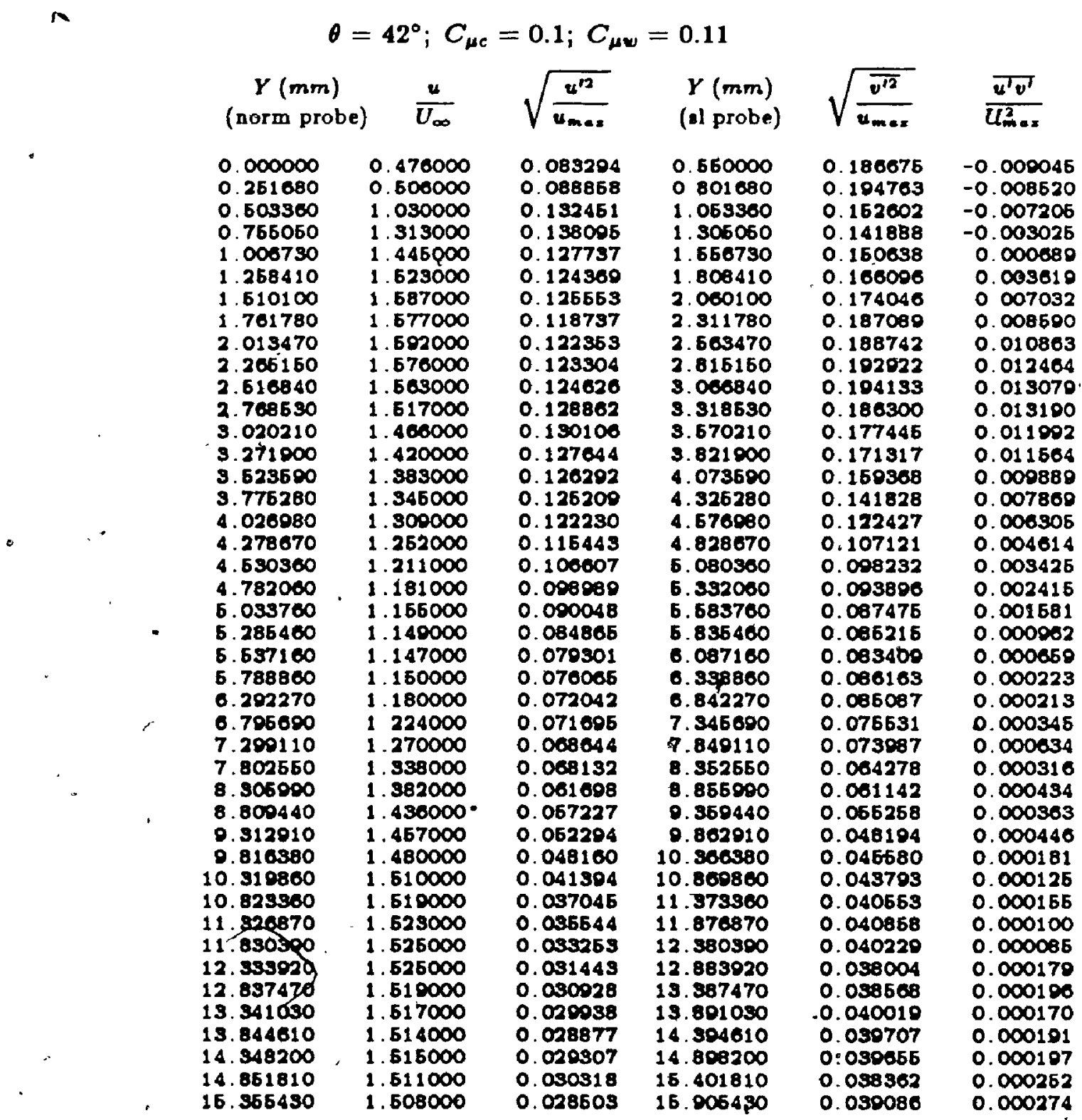


Table G.8: Results from B.L.C. Apparatus Experiments

$$
\theta=42^{\circ} ; C_{\mu c}=0.1 ; C_{\mu w}=0.11
$$

\begin{tabular}{|c|c|c|c|c|c|}
\hline $\begin{array}{c}Y(\mathrm{~mm}) \\
\text { (norm probe) }\end{array}$ & $\bar{U}_{\infty}$ & $\sqrt{\frac{x^{\prime 2}}{u_{\operatorname{mas}}}}$ & $\begin{array}{c}Y(m m) \\
(a l \text { probe) }\end{array}$ & $\sqrt{\frac{\overline{w^{12}}}{u_{m a x}}}$ & $\frac{\overline{u^{\top} w^{\prime}}}{\overline{u_{m=x}^{2}}}$ \\
\hline 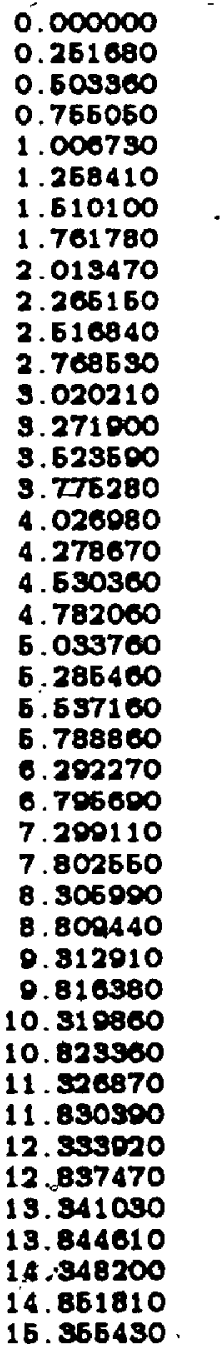 & $\begin{array}{l}0.476000 \\
0.600000 \\
1.030000 \\
1.313000 \\
1.446000 \\
1.623000 \\
1.687000 \\
1.677000 \\
1.692000 \\
1.676000 \\
1.663000 \\
1.617000 \\
1.466000 \\
1.420000 \\
1.383000 \\
1.345000 \\
1.309000 \\
1.262000 \\
1.211000 \\
1.181000 \\
1.165000 \\
1.149000 \\
1.147000 \\
1.160000 \\
1.180000 \\
1.224000 \\
1.270000 \\
1.338000 \\
1.382000 \\
1.430000 \\
1.457000 \\
1.480000 \\
1.610000 \\
1.619000 \\
1.623000 \\
1.625000 \\
1.626000 \\
1.510000 \\
1.617000 \\
1.614000 \\
1.615000 \\
1.611000 \\
1.608000\end{array}$ & 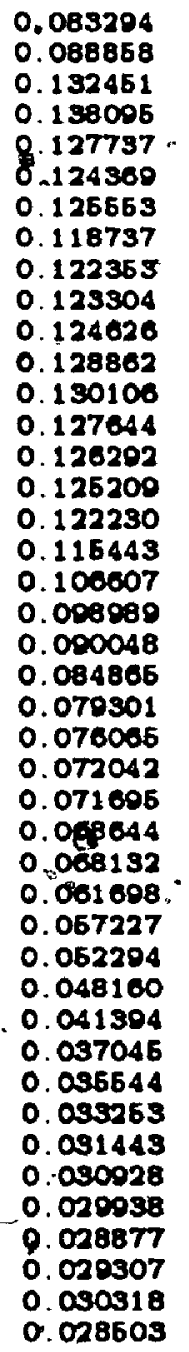 & 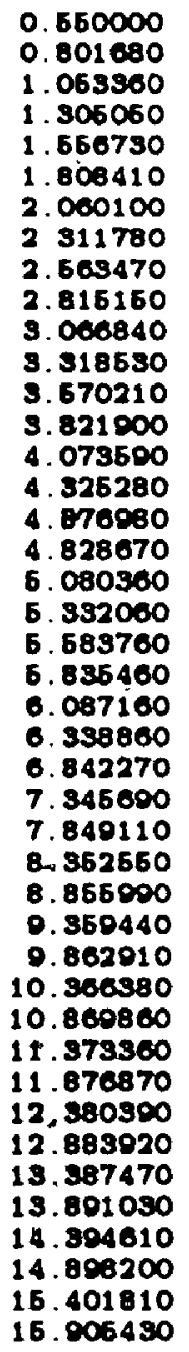 & 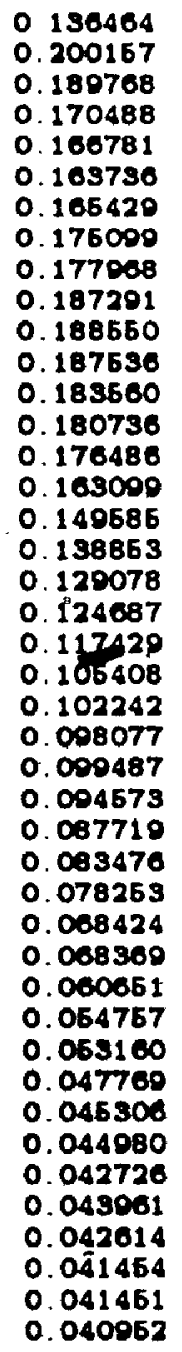 & 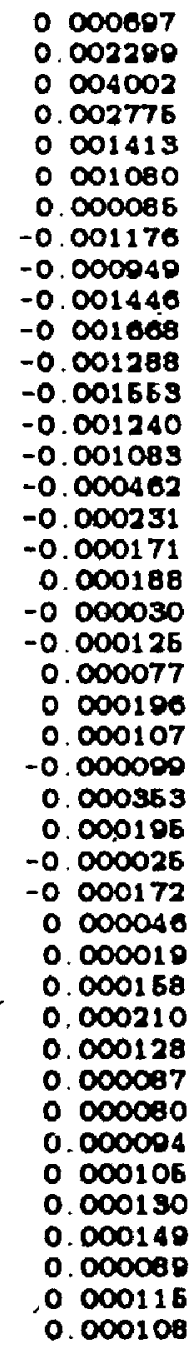 \\
\hline
\end{tabular}


„Table G.9: Results from B.L.C. Apparatus Experiments

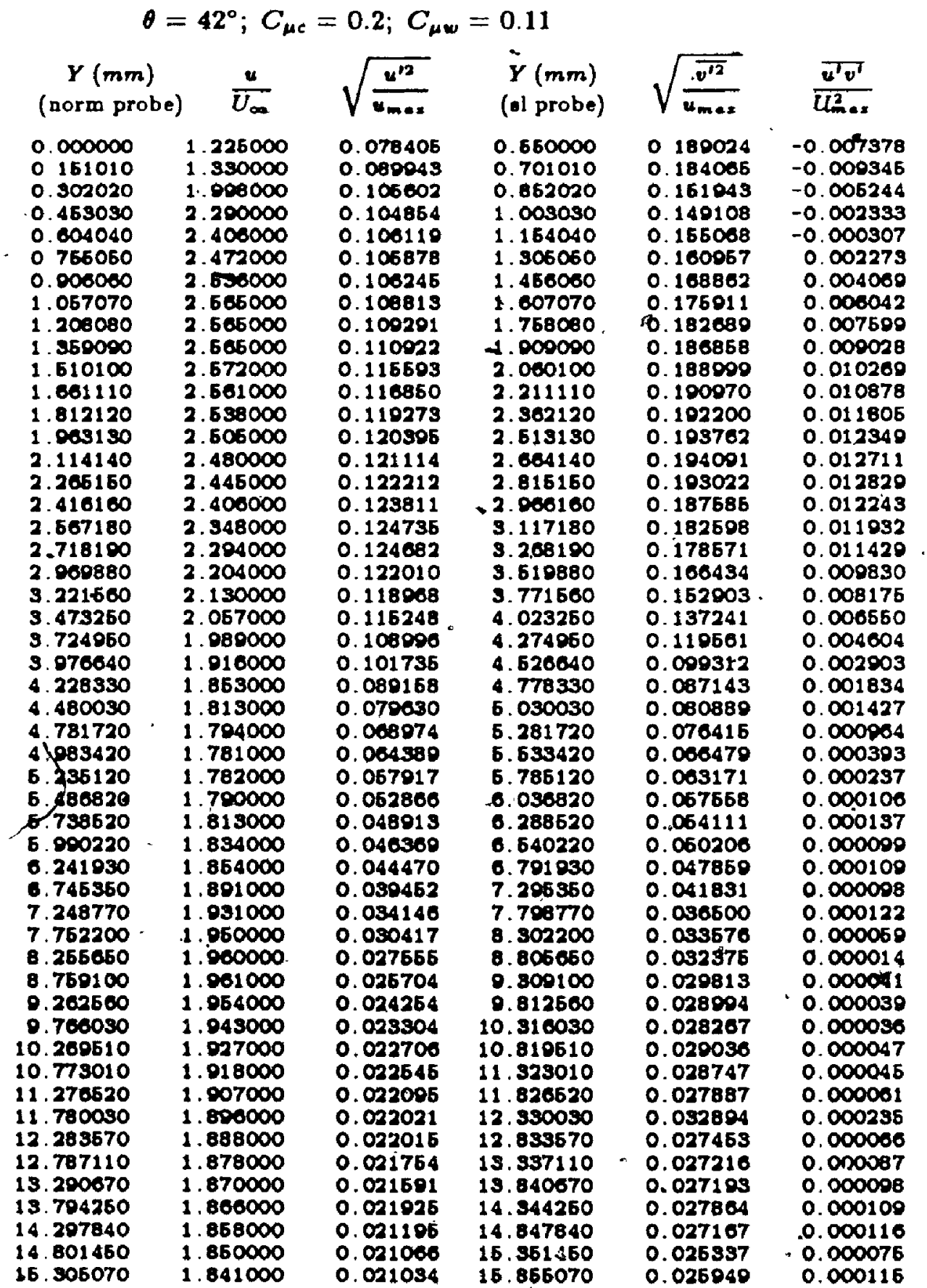

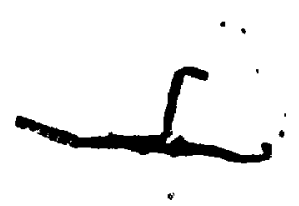


Table G.10: Results from B.L.C. Apparatus -Experiments

$$
\theta=42^{\circ} ; C_{\mu c}=0.2 ; C_{\mu w}=0.11 \quad \text { 。 }
$$

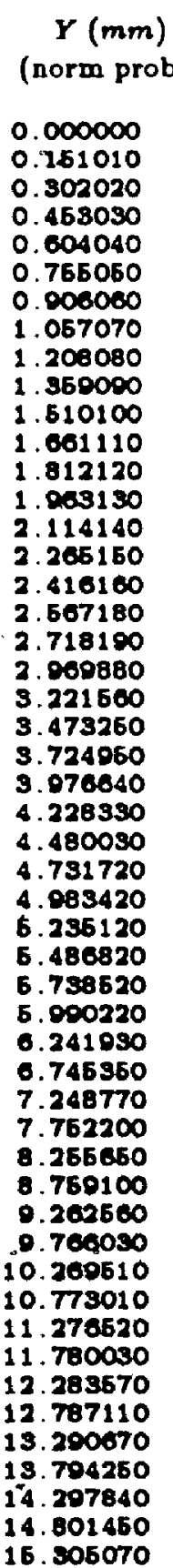

$$
\sqrt{\frac{u^{12}}{u_{m a x}}}
$$

$Y(m m)$
(al probe)

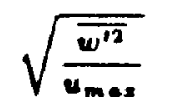

$\frac{\overline{u^{\prime} w}}{U_{m=x}^{2}}$

1.225000

1. 330000

1. 008000

2. 200000

2.400000

2. 472000

2. 636000

2.565000

2.665000

2.666000

2.672000

2.661000

2. 538000

2.605000

2. 480000

2. 446000

2.406000

2. 348000

2. 204000

2. 204000

2. 130000

2. 057000

1.980000

1.010000

1.853000

1. 813000

1. 724000

1.781000

1. 782000

1. 700000

1.813000

1.834000

1. 864000

1. 801000

1. 031000

1. .960000

1. 060000

1. 081000

1.054000

1. 043000

1.927000

1. 918000

1. 007000

1. 800000

1. 888000

1.878000

1.870000

1. 860000

1. 858000

1. 850000

1. 841000
0. 078405

0.089043

0.106602

0.104854

0.100110

0.105878

0.100245

0.108813

0. 100201

0.110022

0.116593

0.116850

0. 119273

0.120996

0.121114

0.122212

0.128811

0.124735

0.124682

0.122010

0.118008

0.115248

0.108000

0.089158

0.079030

0.008974

0.064380

0.067017

0.062866

0.048013

0.046980

0.090452

0.034146

. 0.030417

0.027665

0.025704

0.024254

0.023904

0.022706

0.022546

0.022005

0.022021

0.022016

0.021754

0.021501

0.021025

0.021106

0.021068

0.021034
0.101736

0.044470
0.660000

0.701010

0.862020

1003030

1.164040

1. 305050

$-1.456000$

1.607070

1.758080

1. 000000

2. 060100

2. 211110

2. 302120

2. 613130

2. 004140

2.15150

2. 000160

3. 117180

8. 268100

3. 510880

3.771660

4.023260

4.274060

4.526840

4. 778330

6. 030030

6. 281720

6. 633420

6. 786120

6. 038820

6. 288520

6.640220

- 701030

7.206360

7. 708770

8. 802200

8.806650

0.300100

0.812560

10. 316030

10.819510

11. 323010

11.820520

12. 350090

12.839570

18.397110

$13.840670^{-}$

14.944260

14.847840

16.351450

15. 855070
0.130116

0.146078

0.163082

0.166927

0.166088

0.168587

0.163663

0.167406

0.170345

0.176101

0.176846

0.181138

0.181138
0.183367

0.180085

0.186786

0.187318

o. 186384

0.185124

g. 182868

0.174216

0.161403

0.148056

$0 \quad 134718$

- 0.118211

0. 107742

0.003436

0.081229

0.070503

0.004050

0.001022

0.057130

0.065215

0.040677

0.045004

0.041786

0.030303

0.039716

0.031765

0.030953

0.091040

0.031070

0.020851

0.030018

0.020002

0.020322

0.028760

0.028628

0.020100

0.027830

0.028631

0.028488
$-0002826$

$-0.001110$

- 000146

0000760

0.000503 .

0.000220

0 000298

- 000160

$-0.000116$

$-0.0002501$

$-0.000170$

$-0.000240$

$-0.000213$

$-0.000174$

-0 000202

$-0.000123$

0.000073

- 000010

0.000086

0.000071

0 000538

0.000683

0.000473

0.000564

o. 000862

0,000304

0.000183

- 000356

o. 000180

0.000100

0.000132

0.000112

000001

0.000021

0.000026

0.000035

0.000002

0.000018

$-0.000002$

0.000050

0000002

0.000038

-0, 000008

- 000007

0.000007

0.000020

0.000022

0.000043

0.000008

- 000040

o 000033 
Table G.11: Resulta from B.L.C. Apparatus Experiments

$$
\theta=62^{\circ} ; C_{\mu c}=0.1 ; C_{\mu w}=0.11 \text {. }
$$

\begin{tabular}{|c|c|c|c|c|c|}
\hline $\begin{array}{r}Y(\mathrm{~mm}) \\
\text { (norm prot }\end{array}$ & $\frac{u}{U_{\infty}}$ & $\sqrt{\frac{u^{n}}{u_{\max }}}$ & $\begin{array}{c}Y \text { (mra) } \\
\text { (ol probe) }\end{array}$ & $\sqrt{\frac{\overline{v^{12}}}{u_{\max }}}$ & $\frac{\overrightarrow{u^{\top} v^{\prime}}}{U_{\operatorname{mex}}^{2}}$ \\
\hline 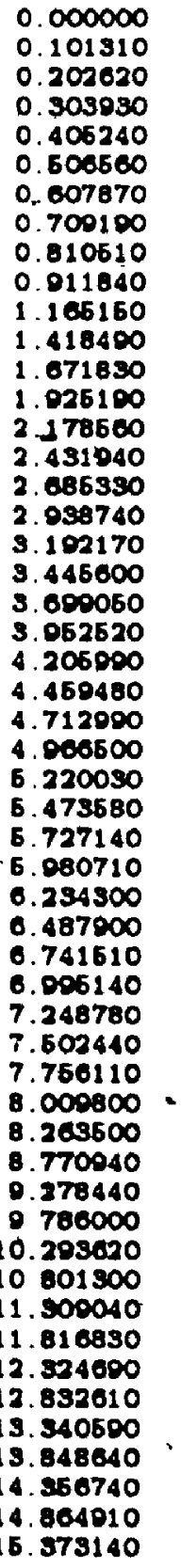 & $\begin{array}{l}0.087000 \\
0.000000 \\
0.090000 \\
0.114000 \\
0.121000 \\
0.133000 \\
0.138000 \\
0.138000 \\
0.146000 \\
0.163000 \\
0.174000 \\
0.186000 \\
0.218000 \\
0.260000 \\
0.284000 \\
0.300000 \\
0.352000 \\
0.381000 \\
0.412000 \\
0.467000 \\
0.449000 \\
0.537000 \\
0.674000 \\
0.674000 \\
0.658000 \\
0.670000 \\
0.762000 \\
0.767000 \\
0.781000 \\
0.808000 \\
0.821000 \\
0.841000 \\
0.850000 \\
0.906000 \\
0.878000 \\
0.891000 \\
0.802000 \\
0.803000 \\
0.003000 \\
0.884000 \\
0.866000 \\
0.863000 \\
0.842000 \\
0.834000 \\
0.811000 \\
0.816000 \\
0.817000 \\
0.825000 \\
0.846000 \\
0.866000 \\
0.000000 \\
0.834000 \\
0.067000\end{array}$ & 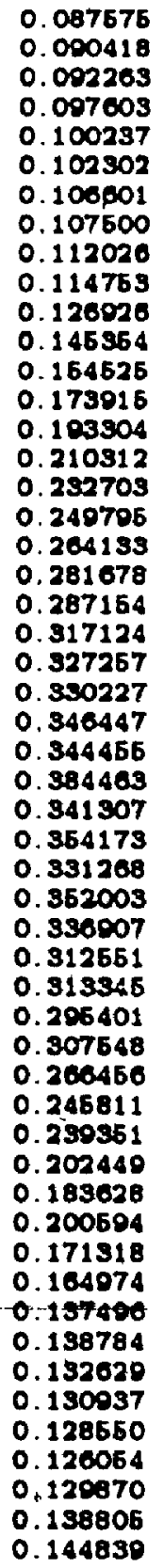 & 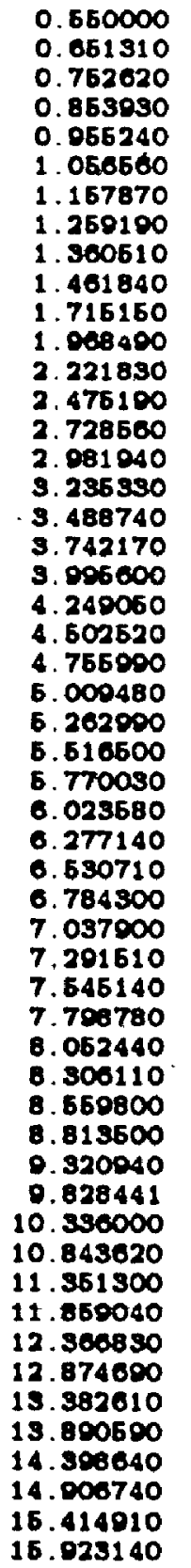 & 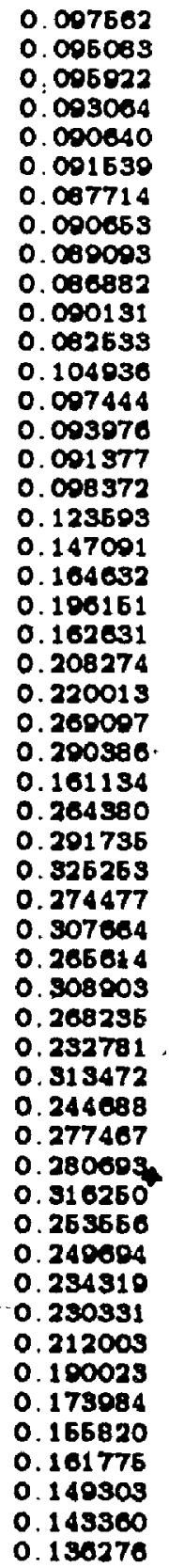 & 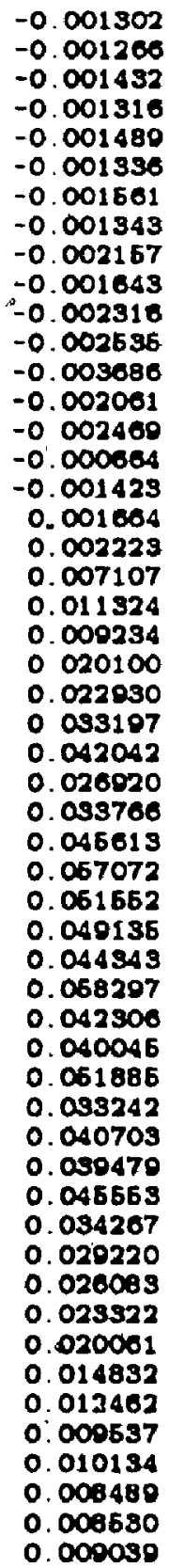 \\
\hline
\end{tabular}


Table G.12: Results from B.L.Ç. Apparatus Experimedłs

$$
\theta=62^{\circ} ; C_{\mu c}=0.2 ; C_{\mu w}=0.11
$$

$Y(\mathrm{~mm})$
(norm probe)

0.000000

0.263270

0.606560

0.750860

1.013160

1.260400

1.610820

1.773170

2.026530

2.279000

2.639300

2.786700

3.040110

3.298540

3.546980

3. 800440

4.063900

4. 307390

4. 560880

4.814390

6. 067910

E. 321460

6. 575000

b. .28560

$6.082: 40$

6. 335730

- .580340

0.842060

7.000600

7.009910

8111280

8.618700

0.126100

0.693730

10. 141330

10. 648000

$11: 156710$

11.604490

12.172330

12. 680200

13. 188100

13.608220

14.204300

14.712460

15.220670 $\sqrt{\frac{. u^{12}}{u_{m \in x}}}$

$Y(\mathrm{~mm})$

(al probe)

$\sqrt{\frac{\overline{v^{n}}}{u_{m a x}}}$

$\frac{\overline{u^{\top} v^{T}}}{u_{\text {mes }}^{2}}$

0.607000

0.803000

0.906000

1.002000

1. 272000

1. 300000

1. 494000

1. 626000

1. 510000

1. 568000

1.530000

1.540000

1.61700C

1.514000

1. 492000

1.470000

1.448000

1. 413000

1. 381000

$1.357 \times 0$

1. 325,60

$1.29,000$

1.27000

1. 243000

1.212000

1.108000

1. 178000

1.107000

1.161000

1.150000

1.161000

1.181000

1.201000

1.236000

1.286000

1. 315000

1.341000

1. 356000

1.368000

1. 377000

1. $\$ 70000$

1. 385000

1. 387000

1. 384000

1. 382000
0. 104659

0. 206060

0.220480

0.227600

0.230143

0.217741

0.220216

0.108608

0.167083

0.161869

0.170708

0.177800

0.160030

0.168777

0.161342

0.165600

0.161680

0.158434

0.156366

U. iᄃ 1902

o. 151420

0. 147266

0.138463

0.135776

0.120581

0.125239

0.110335

0. 113322

0.100500

0.001188

0.084882

0.074847

0.073170

0.067760

0.002606

0.065022

0.062133

0.044617

0.038970

0.055603

0.033746

0.031060

0.031200

0.030080

0.030034
0.660000

- 803270

1.056560

1. 300850

1. 563160

1. 816400

2. 000820

2. 329170

2. 570530

2. 820000

3. 089300

3. 390700

3. 500110

3. 843540

4.000080

4. 350440

4. 603000

4.867390

6. 110880

5. 364390

5. 017910

5. 871460

6.126000

6. 378560

6. 032140

6.885730

7.130340

7.392000

7. 646000

8.153910

8. 601281

0.168700

0.676100

10. 183730

10. 001590

11. 108000

11.700710

12.214400

12.722330

13.230200

13. 738100

14.246220

14.754300

16.262450

16. 770670
-0.01 roto

$0.162441-0.016641$

$0.176703-0.017750$

$0.170460 \quad-0.018766$

$0.212408 \quad-0.021618$

$0.100317-0.018705$

$0.204636 \quad-0.016800$

$0.223826-0.012236$

$0.211862-0.011925$

$0.198112-0007618$

$0.181476 \quad-0.004174$

$0.182447 \quad 0.000784$

$0.186211 \quad-0.000663$

$0.164072 \quad 0.003980$

$0.161566 \quad 0.004535$

$0.167483 \quad 0.004624$

$0.163852 \quad 0.004839$

$0.166393 \quad 0.005010$

$0.152284 \quad 0.006354$

$0.162490 \quad 0.006560$

$0.162811 \quad 0.006140$

$0.165164 \quad 0.004632$

$0.149380 \quad 0.004130$

$0.149106 \quad 0.003652$

$0.162341 \quad 0.004658$

$0.144001 \quad 0.003467$

$0.144077 \quad 0.002676$

$0.135044 \quad 0.001044$

$0.124308 \quad 0.000444$

$\begin{array}{ll}0.110189 & 0.000173\end{array}$

$0.008698 \cdot-0.000472$

$0.085436,-0.000718$

$0.078146 \quad-0.000837$.

$0.070587,-0.001037$

$0.007466 \quad-0001068$

$0.005041 \quad-0,001194$

$0,050892 \quad-0.000045$

$0.068238 \quad-0.00081$.

$0.063266-0.000880$

$0.048243 \quad-0.000806$

$0.046018 \quad-0.000586$

$0.039800 \quad-0.000406$

0.037428 - -0.000369

$0.035813 \quad-0000274$ 
Table G.13: Resulte from B.L.C. Apparatus Experiments

\begin{tabular}{|c|c|c|c|c|c|}
\hline $\begin{array}{c}Y(\mathrm{~mm}) \\
\text { (norm probe) }\end{array}$ & $\frac{u}{U_{\infty}}$ & $\sqrt{\frac{u^{\prime 2}}{u_{m=x}}}$ & $\begin{array}{c}Y(\mathrm{~mm}) \\
\text { (al probe) }\end{array}$ & $\sqrt{\frac{v^{12}}{u_{\max }}}$ & $\frac{\overline{u^{\prime} v^{\prime}}}{\overline{u_{m=x}^{2}}}$ \\
\hline 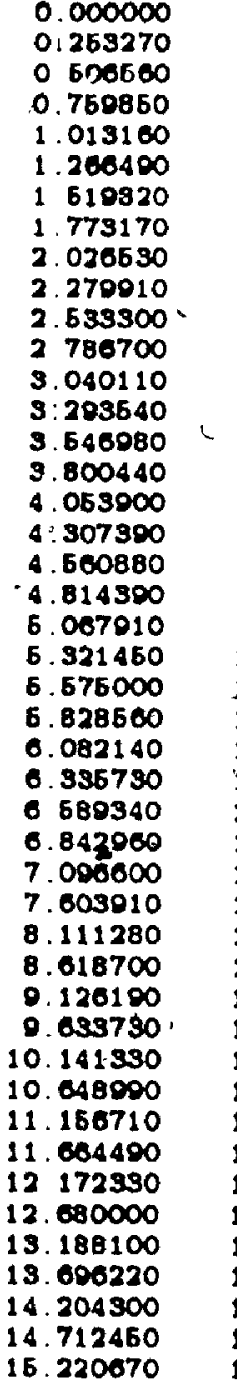 & $\begin{array}{l}0.937000 \\
1.161000 \\
1.204000 \\
1.436000 \\
1.639000 \\
1.636000 \\
1.706000 \\
1.701000 \\
1.700000 \\
1.720000 \\
1.700000 \\
1.688000 \\
1.662000 \\
1.639000 \\
1.617000 \\
1.602000 \\
1.562000 \\
1.507000 \\
1.475000 \\
1.430000 \\
1.407000 \\
1.381000 \\
1.354000 \\
1.320000 \\
1.302000 \\
1.292000 . \\
1.274000 \\
1.266000 \\
1.252000 \\
1.247000 \\
1.283000 \\
1.305000 \\
1.330000 \\
1.949000 \\
1.385000 \\
1.411000 \\
1.422000 \\
1.418000 \\
1.425000 \\
1.432000 \\
1.426000 \\
1.420000 \\
1.430000 \\
1.431000 \\
1.427000\end{array}$ & 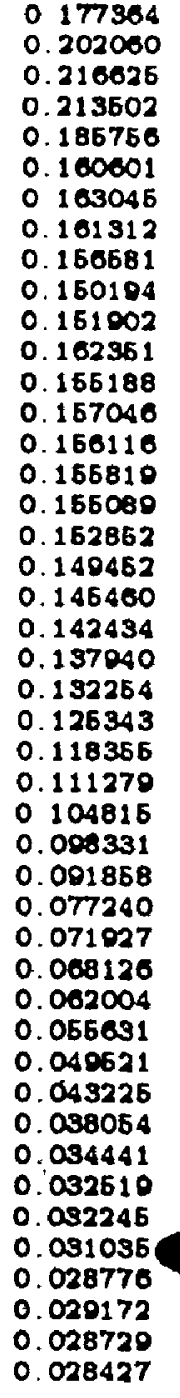 & 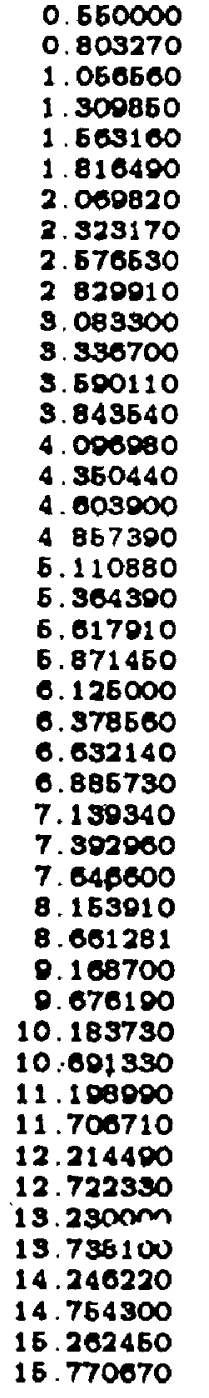 & $\begin{array}{l}0.243085 \\
0.256120 \\
0.223288 \\
0.211048 \\
0.232780 \\
0.212612 \\
0.204140 \\
0.205064 \\
0.204734 \\
0.200813 \\
0.206577 \\
0.200366 \\
0.203074 \\
0.100880 \\
0.201124 \\
0.208202 \\
0.205300 \\
0.206471 \\
0.200921 \\
0.200113 \\
0.104077 \\
0.188568 \\
0.186000 \\
0.177388 \\
0.173242 \\
0.160009 \\
0.161028 \\
0.165682 \\
0.140271 \\
0.130665 \\
0.116761 \\
0.099472 \\
0.003951 \\
0.082348 \\
0.082877 \\
0.071647 \\
0.060088 \\
0.067183 \\
0.066721 \\
0.050783 \\
0.047438 \\
0.047650 \\
0.043842 \\
0.044830 \\
0.042711\end{array}$ & $\begin{array}{l}-0.026248 \\
-0.020830 \\
-0.023860 \\
-0.021443 \\
-0.021711 \\
-0.014032 \\
-0.011463 \\
-0.000277 \\
-0.006100 \\
-0.000811 \\
0.000686 \\
0.001441 \\
0.004362 \\
0.007448 \\
0.008404 \\
0.009086 \\
0.009080 \\
0.010102 \\
0.000760 \\
0.009760 \\
0.009152 \\
0.000034 \\
0.008475 \\
0.007643 \\
0.006725 \\
0.006305 \\
0.005310 \\
0.004261 \\
0.003612 \\
0.002208 \\
0000843 \\
-0.000003 \\
-0.000486 \\
-0.001050 \\
-0.001349 \\
-0.001066 \\
-0.000891 \\
-0.000096 \\
-0.000690 \\
-0.000628 \\
-0.000500 \\
-0.000465 \\
-0.000363 \\
-0.000468 \\
-0.000365\end{array}$ \\
\hline
\end{tabular}


Table G.14: Results from B.L.C. Apparatus Experiments

$$
\theta=72^{\circ} ; C_{\mu c}=0.2 ; C_{\mu \omega}=0.11
$$

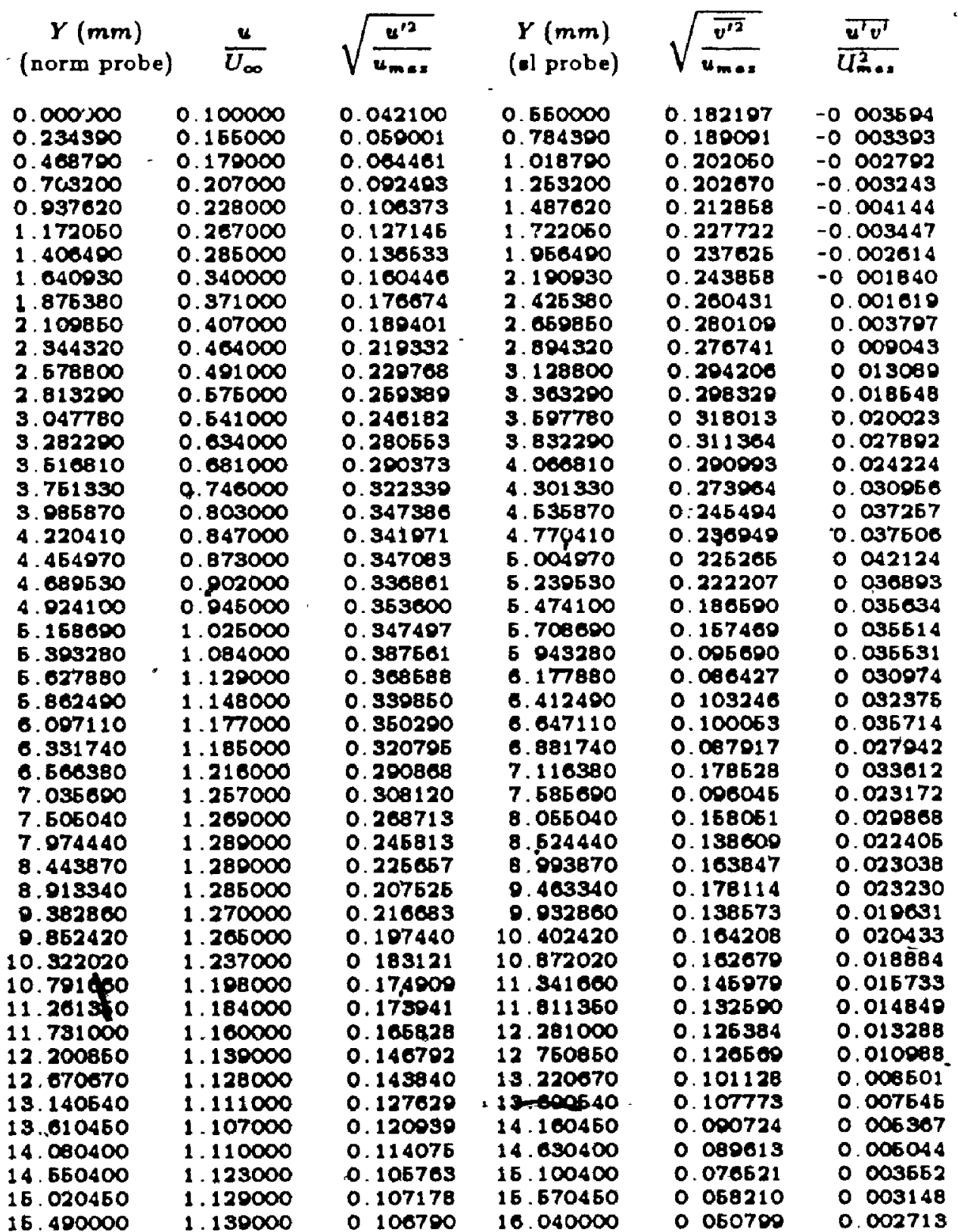


Table G.15: Results from B.L.C. Apparatus Experiments .

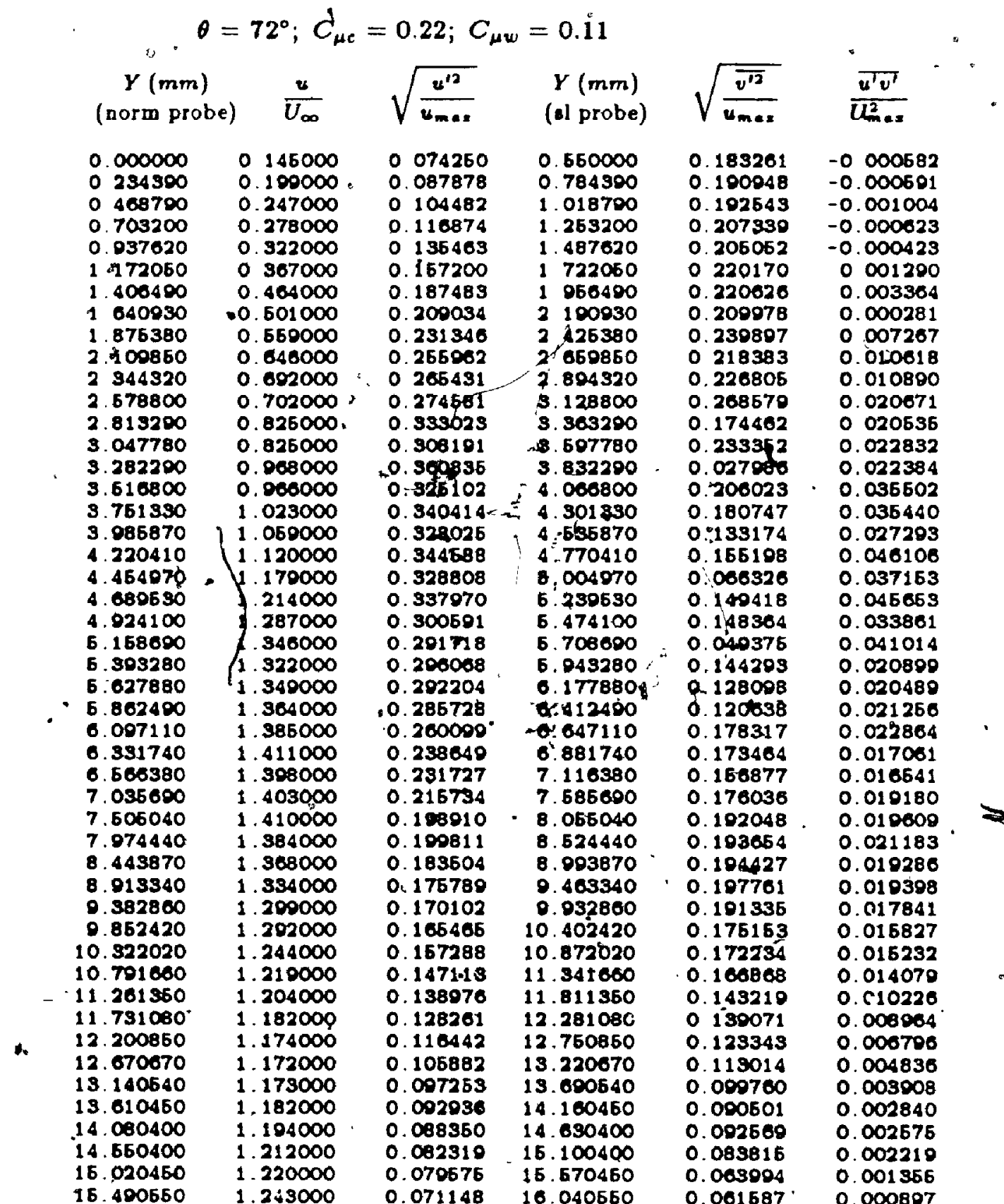

400650 
3

$\because$

1

$$
.7
$$
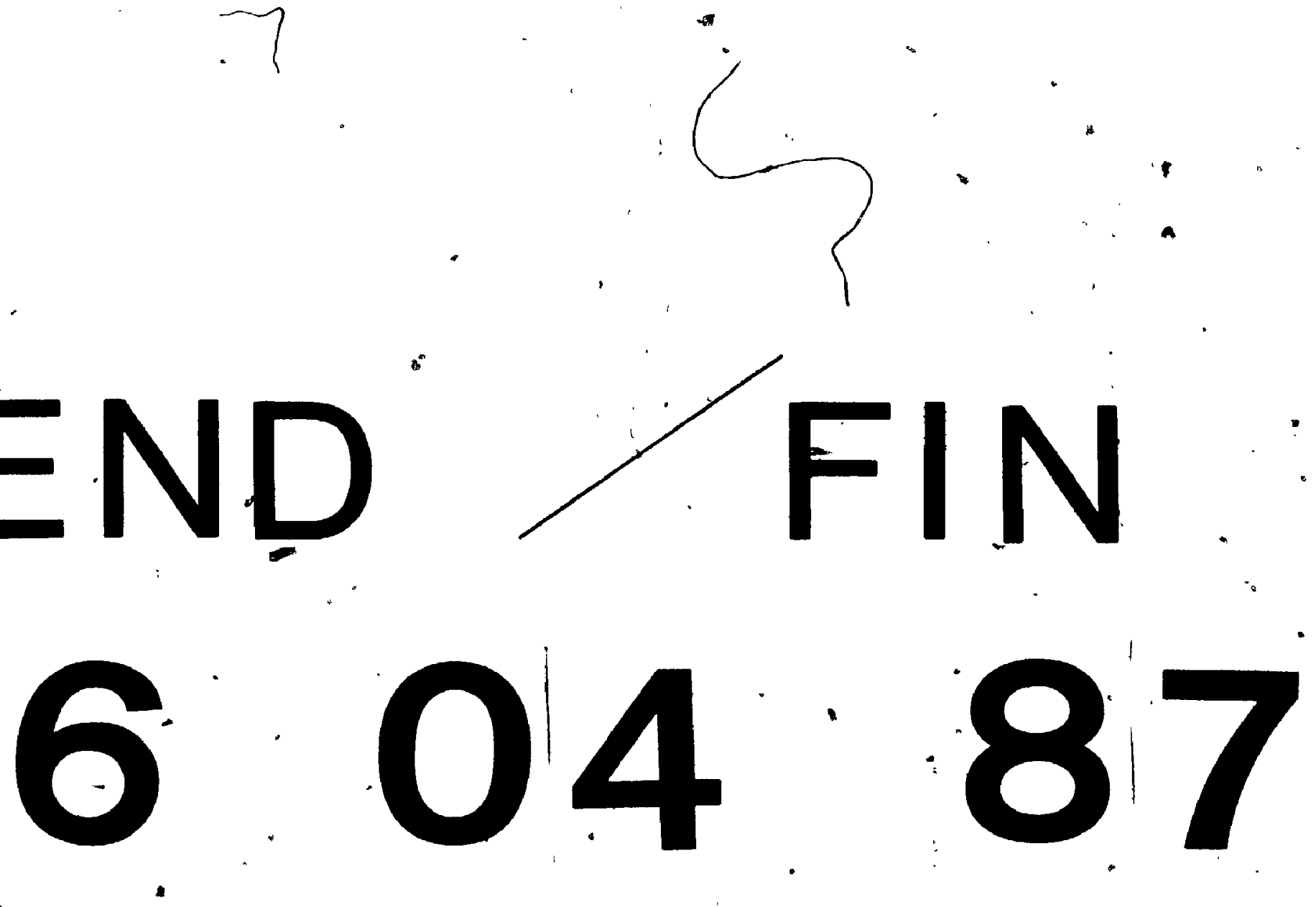

.

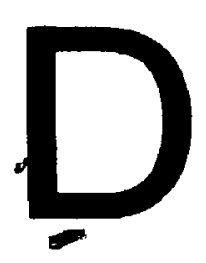

.

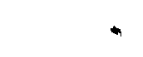

.

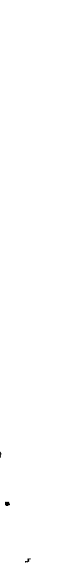

,

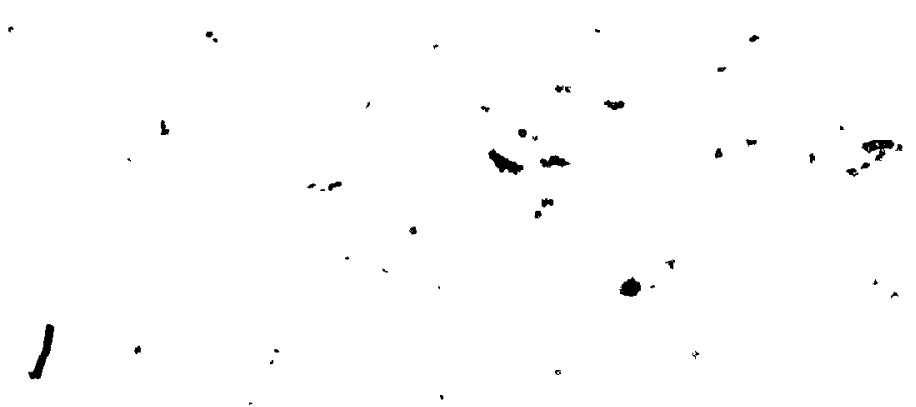

